\title{
AUTOMATED ROUNDABOUT SAFETY ANALYSIS: DIAGNOSIS AND REMEDY OF SAFETY PROBLEMS
}

\author{
by \\ Haytham Sadeq \\ B.Sc., Qatar University, 2009 \\ A THESIS SUBMITTED IN PARTIAL FULFILLMENT OF \\ THE REQUIREMENTS FOR THE DEGREE OF \\ MASTER OF APPLIED SCIENCE \\ in \\ THE FACULTY OF GRADUATE STUDIES \\ (Civil Engineering) \\ THE UNIVERSITY OF BRITISH COLUMBIA \\ (Vancouver) \\ March 2013 \\ (C) Haytham Sadeq, 2013
}




\section{Abstract}

The safety of a transportation system is a serious concern for transportation agencies and analysts. In Canada, roughly $29 \%$ and $43 \%$ of fatalities and serious injury collisions, respectively, occur at intersections (Road Safety Directorate, 2007). There has been a growing interest in the construction of roundabouts to improve the safety performance and increase the traffic efficiency at regular intersections. As more roundabouts are installed throughout North America, there will be an increased need for a detailed analysis of their safety performance. Collision data used to evaluate the safety performance of roundabouts is considered a reactive and costly approach. Recently, the Traffic Conflict Technique (TCT) has been used to improve and complement the collision-based safety diagnosis approach. The purpose of this thesis is to demonstrate the use of an automated safety analysis tool, developed at the University of British Columbia (UBC), for the diagnosis of safety issues at roundabouts. Traffic conflicts occurring at a roundabout, located at UBC campus, are automatically identified and analyzed to develop an in-depth understanding of the behaviour of road users and the causes of traffic conflicts. The results from this detailed and low-cost approach are used to propose effective countermeasures to proactively improve the safety of roundabouts, and to ultimately reduce collisions. Based on these results, the following safety concerns have been determined; a confusion of the right-ofway between entering and circulating vehicles; inappropriate negotiation between circulating and exiting vehicles; higher risk of pedestrian-vehicle conflicts at exit lanes than entry lanes and the accommodation of cyclists at mixed traffic roundabouts. Several countermeasures proposed to address these concerns are to add cross hatch markings, narrow down circulating lanes, modify central island markings, provide pedestrian crossing signs, and propose further education for drivers on using roundabouts and accommodating vulnerable road users. This thesis helps to 
demonstrate the effectiveness of the advanced safety tool in diagnosing safety, and proactively demonstrate safety issues at the roundabout. 


\section{Table of Contents}

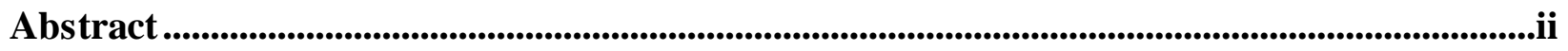

Table of Contents ..........................................................................................................................iv

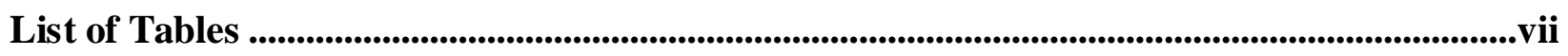

List of Figures.......................................................................................................................... viii

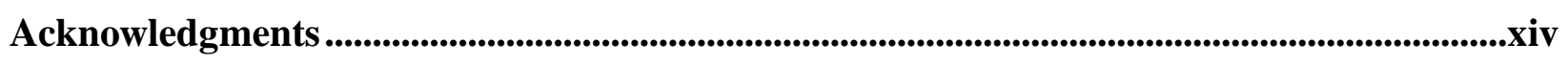

1 Introduction ....................................................................................................................1

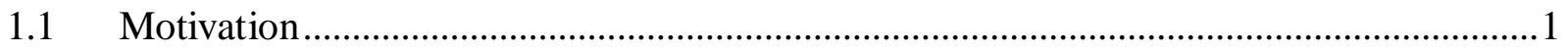

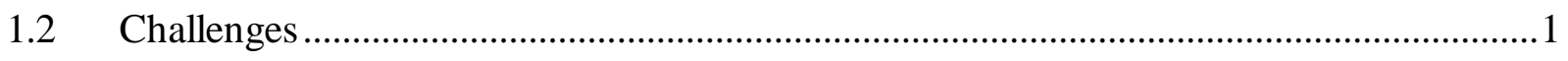

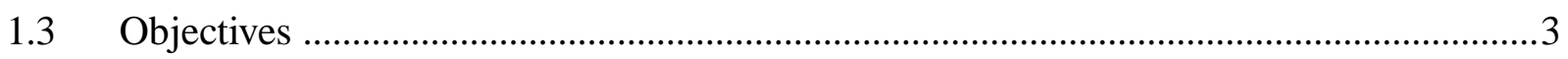

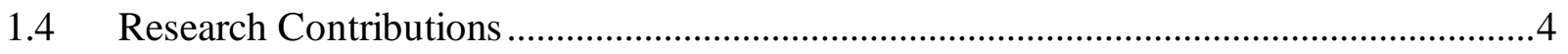

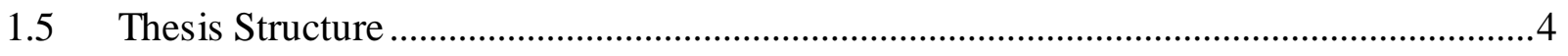

2 Previous Work ................................................................................................................................................5

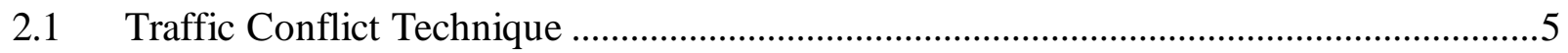

2.1.1 Theory and Technique Developments...................................................................

2.1.2 Objective Conflict's Indicators .............................................................................6

2.1.3 Time-to-Collision Development .............................................................................

2.1.4 Automation of Video Traffic Tracking and Safety Measures ..................................10

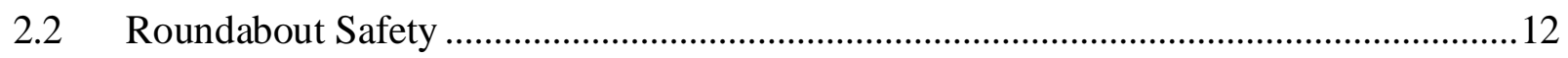

2.2.1 Roundabouts Versus Intersections.................................................................... 12

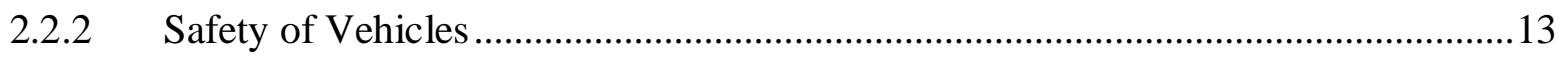

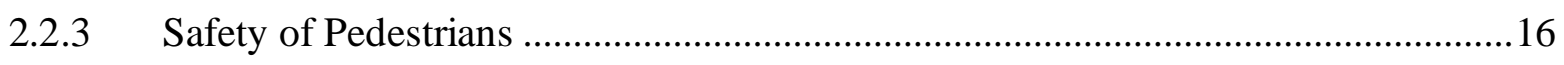

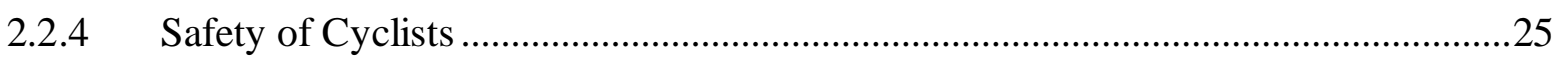

3 Methodology and Data Collection ................................................................................................31

3.1 The Safety Diagnosis Process....................................................................................

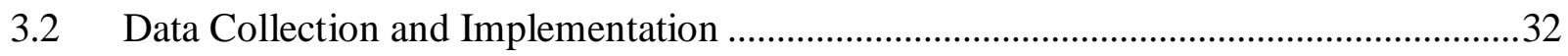

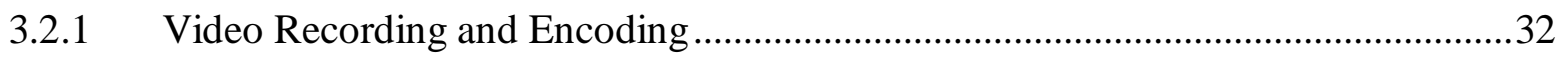

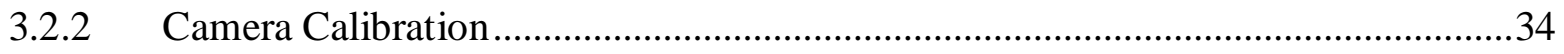

3.2.3 Background Generation.........................................................................................

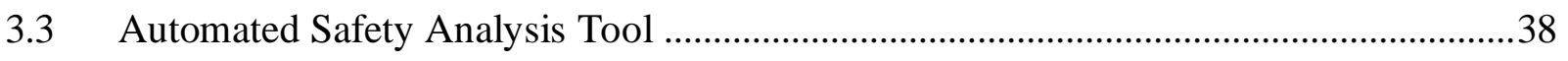




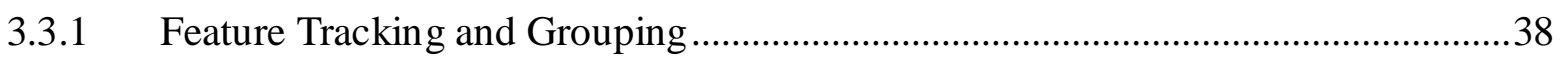

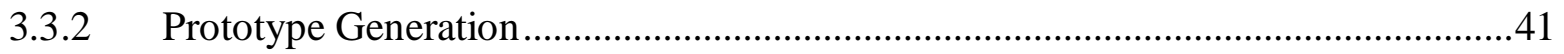

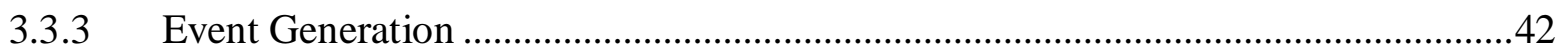

4 Results and Discussion ..................................................................................................................................44

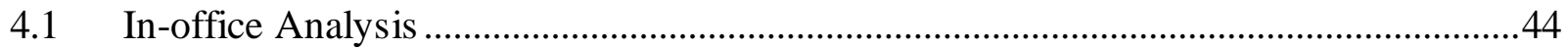

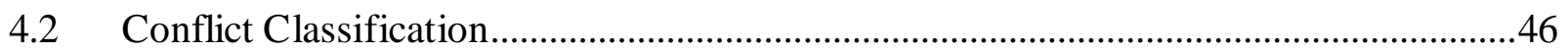

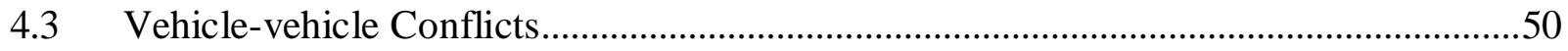

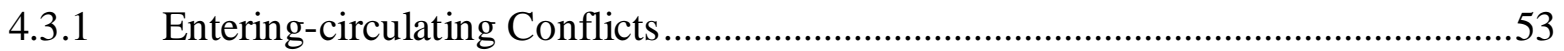

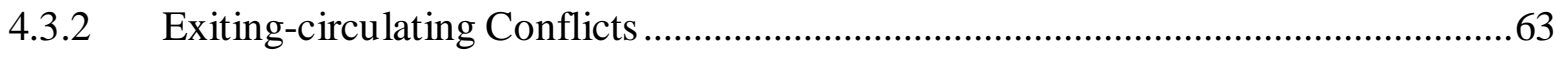

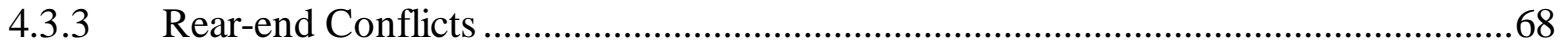

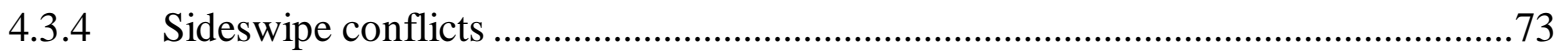

4.3.5 Violations and wrong negotiations of vehicle-vehicle interactions.........................74

4.3.6 Overall Assessment of All Vehicle-vehicle Conflicts:..........................................76

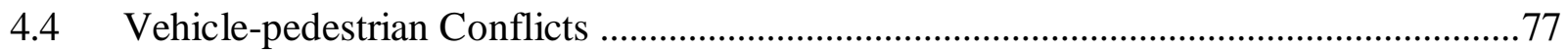

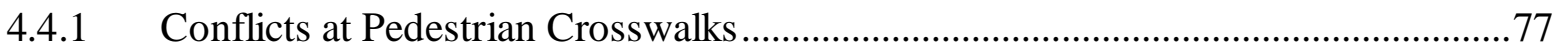

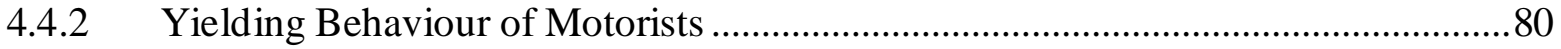

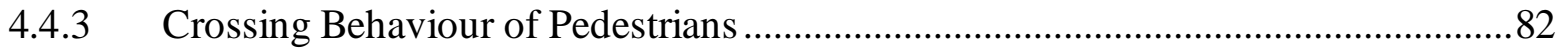

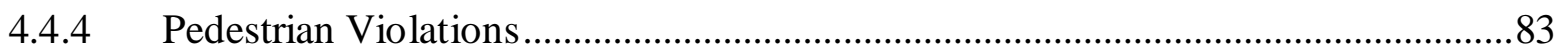

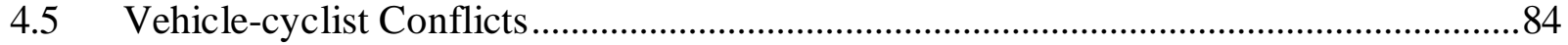

4.5.1 Conflicts of Cyclists Behaving as Vehicles .......................................................... 86

4.5.2 Conflicts of Cyclists Behaving as Pedestrians .......................................................91

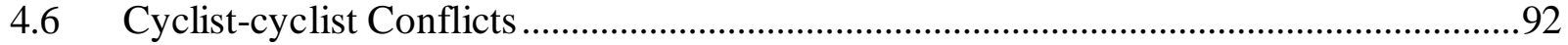

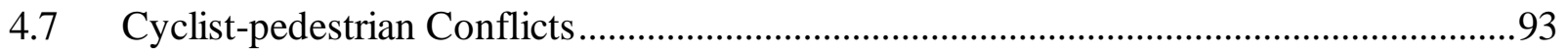

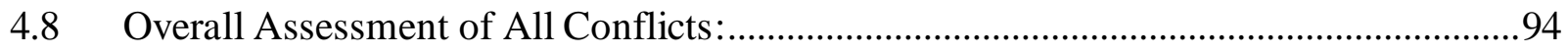

4.9 Conflicts and Accidents Contributory Factors ….........................................................95

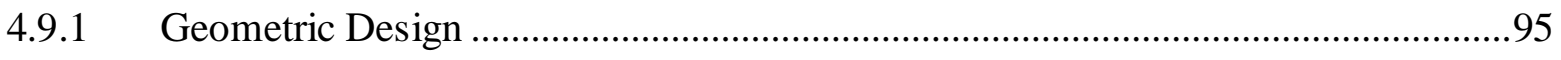

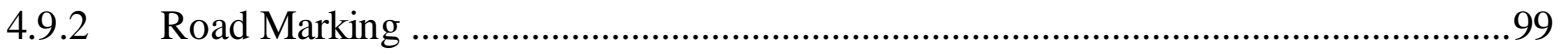

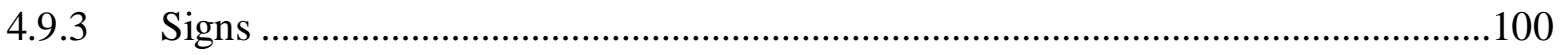

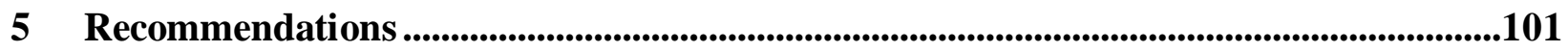

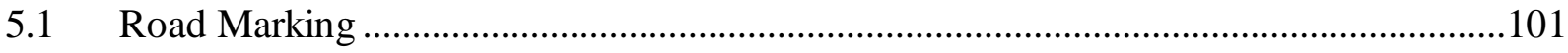

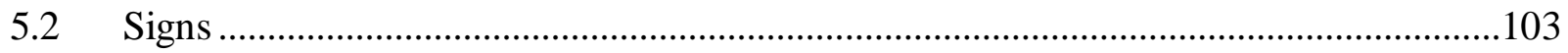




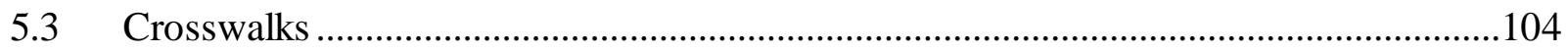

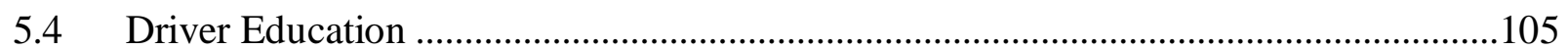

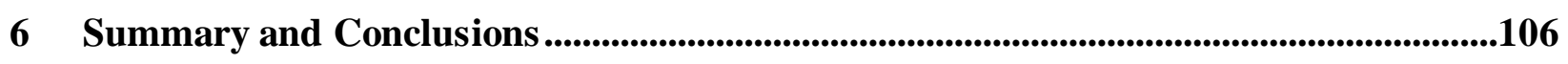

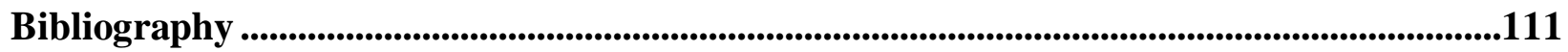

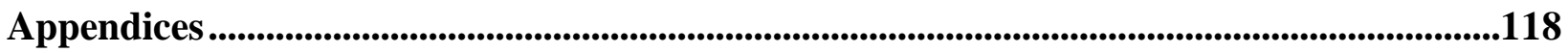

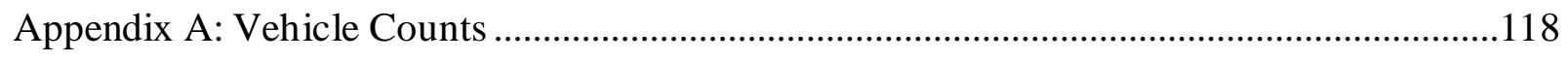

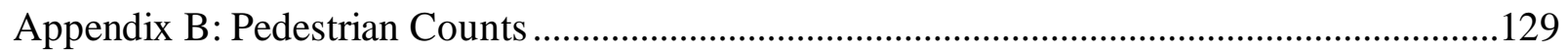




\section{List of Tables}

Table 2.1 Summary of before and after studies of conversions from intersections to roundabouts

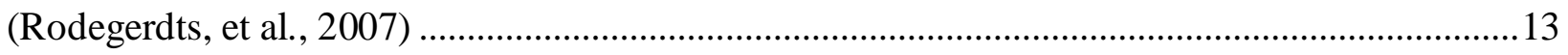

Table 3.1 Video recording periods and details for the five cameras ..........................................33

Table 3.2 List of video components details for scene 1 in day 1 .................................................

Table 4.1 Descriptions and figures of conflict types..........................................................51

Table 4.2 Comparison of the entering-circulating conflicts in approaches A, B, C, and D. .........55

Table 4.3 Comparison of the exiting-circulating conflicts in approaches A, B, C and D............63

Table 4.4 Comparison of rear-end conflicts in approaches A, B, C and D...............................69

Table 4.5 Weighted average values for conflict rates across conflict's types ...............................77

Table 4.6 Comparison of vehicle-pedestrian conflict rates at the entry and exit lanes of

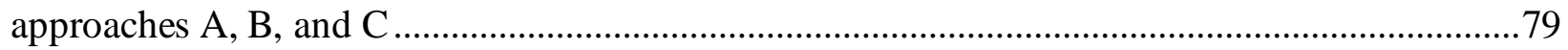

Table 4.7 Yielding behaviour of motorists and cyclists in entering-circulating conflicts ............89

Table 6.1 Safety concerns found in the analysis and the proposed countermeasures.................108 


\section{List of Figures}

Figure 2.1 The pyramid - the interaction between road users as a continuum of events proposed by Hydén (1987). .5

Figure 2.2 Example of three interactions among three road users in four potential conflict points (Saunier, Sayed, \& Ismail, 2010). 9

Figure 2.3 The main roundabout geometric design parameters (Montella A. , 2011) ..................14

Figure 2.4 Difference between contributing factors and collision causes 15

Figure 2.5 Offset approach alignment to increase deflection angle and reduce vehicle entry speed

Figure 2.6 Offset in crosswalk between entry and exit legs (Baranowski, 2005) .20

Figure 2.7 Threshold chart to apply pedestrian crossing signal for (a) four-hour volume, and (b) one-hour volume (FHWA, 2009) .22

Figure 2.8 The distant signal pedestrian crossing layout (Lu, Guan, \& Noyce, 2010) .23

Figure 2.9 Four basic types of cycle facilities: (a) mixed traffic; (b) cycle lane within the roundabout; (c) separate cycle path; and (d) grade-separated cycle paths (Daniels, Brijs, Nuyts, \& Wets, 2009). .28

Figure 2.10 Two types of cycle facilities: (a) a separated roundabout (with a separate cycle path); and (b) an integrated roundabout (mixed traffic). 29

Figure 3.1 The focus area of the five cameras scenes .33 Figure 3.2 Feature points and distance lines used to link (a) the video image, with (b) the world image (maps data, Google, 2011). .36 Figure 3.3 Use of a square grid to validate the calibration process between (a) the video image, and (b) the world image. .37 
Figure 3.4 Example of backgrounds generated (scene one, day one) with six backgrounds for each one-hour video .38

Figure 3.5 Example of feature tracking of the second scene video component 39

Figure 3.6 Example of feature grouping of a vehicle in a video component of scene two .40

Figure 3.7 Example of mask image used in second scene to focus on vehicle-vehicle interactions

Figure 3.8 Example of prototypes generated for scene one (day one) for vehicle-pedestrian conflicts plotted on (a) the video image, and (b) the world image.

Figure 4.1 In-office analysis and the major issues found by the preliminary safety analysis of the roundabout .44

Figure 4.2 Volume count for vehicles and pedestrians (9:00 am to 8:00 pm - two days)............45

Figure 4.3 Volume count for vehicles and pedestrians as noted by arrows (two days)................46

Figure 4.4 Frequency of different conflict types ..............................................................48

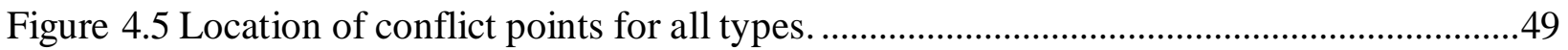

Figure 4.6 The heat map of all conflict points per area............................................................50

Figure 4.7 Frequency of each vehicle-vehicle conflict types.................................................52

Figure 4.8 Conflict points of all vehicle-vehicle conflicts...................................................52

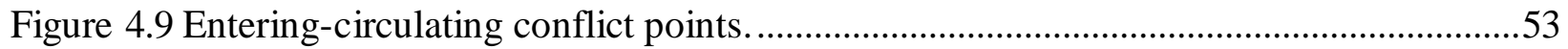

Figure 4.10 Volumes of entering and circulating vehicles (two days) on approach C................54

Figure 4.11 Volumes of entering and circulating vehicles (two days) for approaches A, B, C and D. .55

Figure 4.12 Comparison of the entering-circulating conflicts in approaches A, B, C, and D......56

Figure 4.13 Entering-circulating conflicts and vehicles' behaviour on approaches A and B. .......58 
Figure 4.14 A Yield sign of traffic in roundabout when entering vehicles from approach A.......58 Figure 4.15 Entering-vehicle conflicts and vehicles' behaviour in gap acceptance on approach A and $\mathrm{B}$

Figure 4.16 An entering-circulating conflict and vehicles' behaviour on approach D.................60

Figure 4.17 Entering-circulating conflicts and vehicles' behaviour on approach C....................61

Figure 4.18 Entering-circulating conflicts TTC distribution normalized to exposure for approaches A, B, C and D.

Figure 4.19 Entering-circulating conflicts severity distribution normalized to exposure for approaches A, B, C and D

Figure 4.20 Comparison of the exiting-circulating conflicts in approaches A, B, C and D........64

Figure 4.21 Exiting-circulating conflicts and vehicles' behaviour in a negotiation on approach B.

Figure 4.22 Exiting-circulating conflicts and vehicles' behaviour in negotiation on approach C.66

Figure 4.23 Exiting-circulating conflicts and vehicles' behaviour in a negotiation on approach D.

Figure 4.24 Exiting-circulating conflicts TTC distribution normalized to exposure for approaches

A, B, C and D.

Figure 4.25 Exiting-circulating conflict severity distribution normalized to exposure for approaches A, B, C and D. .68

Figure 4.26 Comparison between rear-end conflicts in approaches A, B, C and D....................70

Figure 4.27 Entering rear-end conflicts and vehicles' behaviour in all approaches..................... 71

Figure 4.28 Entering rear-end conflicts and vehicles' behaviour in all approaches......................72

Figure 4.29 Exiting vehicles turning right past the pedestrian crosswalk on approach C............72 
Figure 4.30 Sideswipe conflict points

Figure 4.31 Example of sideswipe conflicts .74

Figure 4.32 Violation of a vehicle entering from approach $\mathrm{C}$ and turning left in error. .75

Figure 4.33 A circulating vehicle yields in error for an entering vehicle. .75

Figure 4.34 A circulating vehicle yields in error for an entering vehicle mistake .........................76

Figure 4.35 Example of a vehicle-pedestrian conflict....................................................................77

Figure 4.36 Frequency of vehicle-pedestrian conflicts at the entry and exit lanes of approaches A,

$\mathrm{B}$ and $\mathrm{C}$ .78

Figure 4.37 Comparison of vehicle-pedestrian conflict rates at entry and exit lanes of approaches

$\mathrm{A}, \mathrm{B}$, and $\mathrm{C}$. .80

Figure 4.38 Yielding behaviour of motorists when pedestrians are crossing .81

Figure 4.39 Crossing behaviour of pedestrians when a vehicle is present .83

Figure 4.40 Pedestrian spatial violations in crossing in the exit lane on approach C. .84

Figure 4.41 The road marking placed before the roundabout to direct cyclists. .85

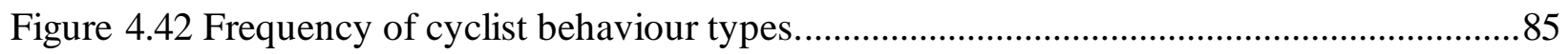

Figure 4.43 Frequency of vehicle-cyclist conflicts types...............................................................86

Figure 4.44 Vehicle-cyclist conflicts when cyclists behave as vehicles. ........................................87

Figure 4.45 Examples of entering-circulating vehicle-cyclist conflicts.........................................8

Figure 4.46 Examples of rear-end (entering) vehicle-cyclist conflicts. ..........................................89

Figure 4.47 Examples of rear-end (exiting) vehicle-cyclist conflicts. ............................................90

Figure 4.48 Examples of rear-end (circulating) vehicle-cyclist conflicts. .......................................90

Figure 4.49 Examples of sideswipe vehicle-cyclist conflicts. ........................................................91 
Figure 4.50 Vehicle-cyclist conflicts when (a) a cyclist is crossing from island, and (b) a cyclist is crossing from side.

Figure 4.51 Examples of cyclist-cyclist conflicts. .93

Figure 4.52 Examples of cyclist-pedestrian conflicts. .94

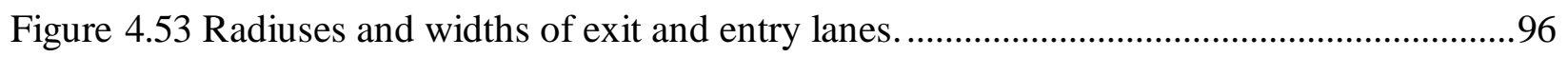

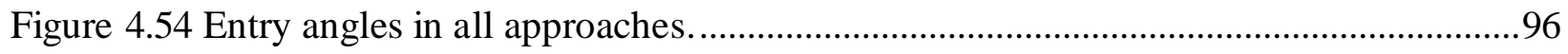

Figure 4.55 Radius of deflection for all entering approaches ..................................................97

Figure 4.56 Deflection angles for all entering approaches. ..................................................98

Figure 4.57 Sight distance check for approaches A and C. ...................................................99

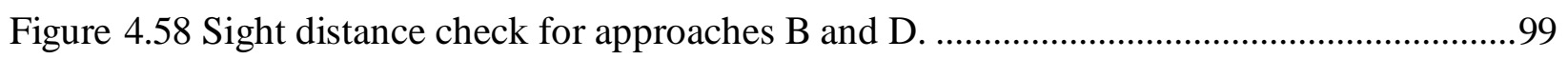

Figure 4.59 Faded or poorly designed road markings..........................................................100

Figure 5.1 Proposed road markings modifications............................................................... 101

Figure 5.2 Suggested signs at multi-lane roundabouts as per the MUTCD (FHWA, 2009).......104

Figure 5.3 Suggested landscape on the approach sides....................................................... 105

Figure A.1 Circulating vehicle volumes for approach A from 9:00 am to $8 \mathrm{pm} \mathrm{a)} \mathrm{Day} \mathrm{one,} \mathrm{and} \mathrm{b)}$

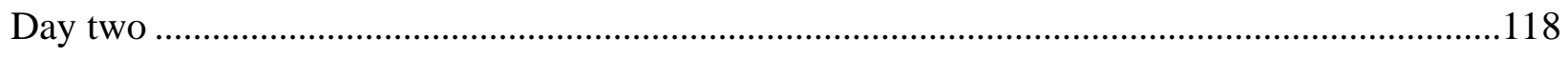

Figure A.2 Circulating vehicle volumes for approach B from 9:00 am to 8 pm a) Day one, and b)

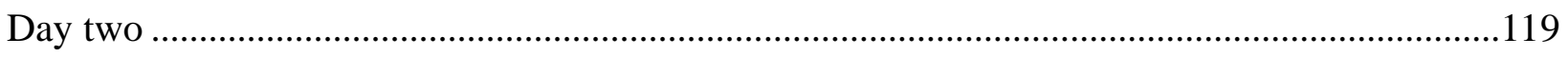

Figure A.3 Circulating vehicle volumes for approach C from 9:00 am to 8 pm a) Day one, and b)

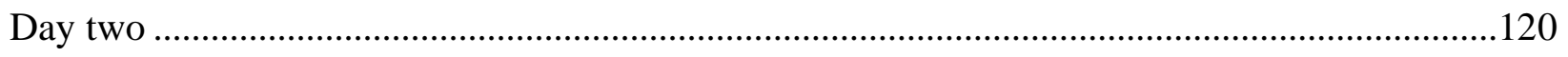

Figure A.4 Circulating vehicle volumes for approach D from 9:00 am to 8 pm a) Day one, and b)

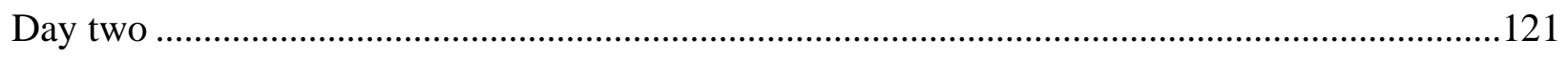


Figure A.5 Entering vehicle counts for approach A (lane one and two) from 9:00 am to 8:00 pm

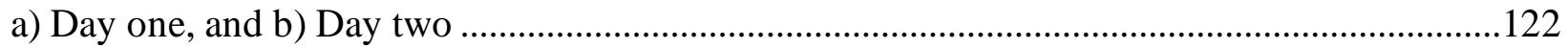

Figure A.6 Exiting vehicle counts for approach A (lane one and two) from 9:00 am to 8:00 pm a)

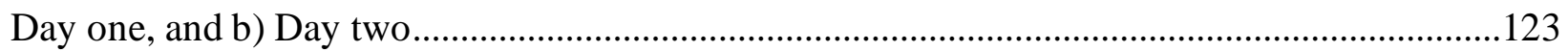

Figure A.7 Entering vehicle counts for approach B (lane one and two) from 9:00 am to 8:00 pm

a) Day one, and b) Day two .124

Figure A.8 Exiting vehicle counts for approach B (lane one and two) from 9:00 am to 8:00 pm a) Day one, and b) Day two. .125

Figure A.9 Entering vehicle counts for approach C lane 1 from 9:00 am to 8:00 pm a) Day one, and b) Day two. 126

Figure A.10 Exiting vehicle counts for approach C lane 1 from 9:00 am to 8:00 pm a) Day one, and b) Day two. .127

Figure A.11 Entering vehicle counts for approach D lane 1 from 9:00 am to 8:00 pm a) Day one, and b) Day two. .128

Figure B.1 Crossing pedestrians at approach A beginning from entry lane and from exit lane from 9:00 am to 8:00 pm a) Day one, and b) Day two..... .129 Figure B.2 Crossing pedestrians at approach B beginning from entry lane and from exit lane from 9:00 am to 8:00 pm a) Day one, and b) Day two. .130 Figure B.3 Crossing pedestrians at approach $\mathrm{C}$ beginning from entry lane and from exit lane from 9:00 am to 8:00 pm a) Day one, and b) Day two. 131 


\section{Acknowledgments}

I am greatly thankful to Professor Tarek Sayed for his continues guidance and support. I appreciate his boundless academic and social support all through the time that I studied at UBC. It was my honor to work and learn the craft of research from his supervision and teaching.

I would like also to thank Dr. Jinhua Zhao for his academic support all through my courses and research work. I am thankful also for Dr. Mohammed Zaki for his continues guidance and assistance in my research and studies.

I would like to acknowledge the continuous help and support from my colleague Jarvis Auty for his great help all through my research project. I appreciate Jarvis' contribution in technical scripts, tutorials, and data collection. I appreciate the great help done by Ahmed Saleh for his technical scripts and support. I would like to acknowledge also the great efforts done by my colleagues in the research team Aaron Mahiban, Houman Hediyeh, and Mohammed Azab.

I greatly acknowledge the invaluable support from my father Adnan and my mother Samia all through my life. They have been always there for all types of social, educational and academic support. I am proud of them and I hope that they will be proud of me also. My great thanks for my brothers Husam and Mohammed for their great guidance in my life. A great appreciation is to my little sister Lubna for her encouraging and pleasant support.

I am ultimately grateful for Allah (God) for giving me the knowledge, intellect, and great blessings that I will never be able to count. 


\section{Introduction}

\subsection{Motivation}

The sustainability of transportation systems continues to be a concern for transportation agencies and analysts. One specific concern is manifested in issues related to the safety of transportation systems. Worldwide, approximately 1.3 million people are killed in road collisions each year, and many more are injured. In Canada, about $29 \%$ and $43 \%$ of fatalities and serious injury collisions, respectively, occur at intersections (Road Safety Directorate, 2007), and more than 2.4 million road accidents occur at U.S. intersections each year (Insurance Institute for Highway Safety, 2008). Recently, there has been a growing interest in the construction of roundabouts to replace regular intersections. The main objective for constructing roundabouts is to increase the safety and operational efficiency of traffic movements. Several studies have shown that converting intersections to roundabouts results in improving the safety performance of intersections ( (Schoon \& Minnen, 1993); (Rodegerdts, et al., 2007)). As the number of roundabout installations continues to increase in North America, there is a need for a detailed study of their safety performance that includes an analysis of driver behaviour at roundabouts, and potential safety issues.

\subsection{Challenges}

Traditionally, safety diagnosis studies of roundabouts have been undertaken using collision data. However, the reliance on collision data for safety analysis has several shortcomings (Sayed \& Zein, 1999), as there are well-known quality and quantity problems associated with this data. In almost all road jurisdictions, the quality of collision data has degraded over the years. In addition, collisions are rare events which mandate the waiting for long time periods (about 3 years) to accumulate adequate collision data for analysis. The use of collision data is also 
considered a reactive approach as a significant number of collisions need to occur before an action can be taken.

These limitations have raised the importance of using surrogate measures to evaluate the safety of the roundabout, and one of the commonly used surrogate measures in traffic safety is traffic conflicts (near misses). The Traffic Conflict Technique (TCT) (Perkins \& Harris, 1968) depends on observing and evaluating traffic conflicts. A traffic conflict is defined as an event occurring between two or more road users approaching one another in a particular time and space, that can lead to a collision if their movements have not changed (Amundsen \& Hydén, 1977).

Traditionally, traffic conflict techniques relied on human observers to record and evaluate the frequency and severity of traffic conflicts at a particular site. This allows for the evaluation of unsafe geometric conditions and driving behaviour at road locations. In addition, traffic conflicts occur more frequently than collisions and are of little social cost. Therefore, safety evaluations based on traffic conflicts can be conducted over shorter periods. However, the high cost of training observers and collecting the conflicts data, coupled with issues related to the reliability and accuracy of the observers, have been limiting factors that inhibit extensive application of the technique (Sayed, Brown, \& Navin, 1994). Therefore, the successful automation of extracting conflicts from video data can considerably be beneficial for traffic safety studies (Saunier \& Sayed, 2007).

In this study, video sensors are selected as the primary source for traffic conflicts data as video data is rich in details, recording devices are becoming less expensive, and video cameras are often already in place for monitoring purposes (Saunier \& Sayed, 2007). In addition, video data represents a permanent record of analyzed traffic events that can be reviewed and validated, as 
opposed to in-field observer-based surveys. The automated analysis of video data to measure traffic conflicts is undertaken using the UBC automated traffic safety tool (Saunier \& Sayed, 2006). The UBC approach relies on the building of two databases: a trajectory database and an interaction database. The trajectory database contains the results of the video processing module. The interaction database contains all the interactions between the road users (within a given distance, and for which various indicators, including collision probability and other severity indicators) that can be automatically computed. Traffic conflicts can be identified and other traffic parameters measured through mining these databases (Saunier \& Sayed, 2008).

\subsection{Objectives}

The main purpose of this study is to use automated road safety analysis tools to diagnose safety issues at a recently installed roundabout located at the University of British Columbia (UBC). The automated system provides a more objective and detailed analysis that relies on video data.

This research is conducted to achieve the following specific objectives:

- Use the automated video analysis approach to identify and measure the severity of traffic conflicts between vehicles, pedestrians and cyclists at the UBC roundabout.

- Demonstrate the use of the automated safety analysis tool in diagnosing the safety issues at the roundabouts.

- Develop an in-depth understanding of the behaviour of the road users, the causes of traffic conflicts that contribute to the safety issues at the UBC roundabout.

- Recommend countermeasures to improve the safety level of the roundabout. 


\subsection{Research Contributions}

Roundabouts are becoming increasingly popular in North America, and given this popularity, there is a significant need for a better understanding of their safety performance and associated driver behaviour. This research demonstrates the use of automated safety analysis tools to diagnose safety issues. This approach to safety analysis is proactive, as there is no need to wait for collisions to be recorded before an analysis is conducted. Furthermore, this approach should offer a better understanding of vehicle collision failure mechanisms, particularly the collisionavoidance behaviour of drivers, that should help our diagnosis of the safety problems and in the selection of safety countermeasures (Sayed, Brown, \& Navin, 1994). With an understanding of the types and degree of severity of potential collisions from the extracted conflict data, economic analyses can be performed for more precise estimates of the cost savings of mitigated collisions.

\subsection{Thesis Structure}

The first chapter of the thesis describes the motivation for the research, as well as the challenges, objectives, and research contributions. The second chapter provides a comprehensive literature review of the traffic conflict techniques and the roundabout safety issues. Chapter three covers the methodology used in the research and the processing of video data to apply the automated conflict analysis technique. The fourth chapter presents the analysis results and discusses conflicts contributing factors, causes, and associated road user behaviour. Chapter five presents potential safety countermeasures. Finally, chapter six provides the summary and conclusions of the study in addition to future research. 


\section{Previous Work}

This chapter summarizes previous key research studies conducted on the use of the TCT in diagnosing the safety level for roundabouts. The first part of the chapter focuses on the theory of traffic conflicts and its historical development. It also describes the TCT automation process development using computer vision techniques. The second part focuses on the previous work conducted to evaluate the safety at roundabouts. It includes key research done on traffic conflicts at roundabouts and their shortcomings, and discusses traffic conflict studies related to vehicles, pedestrians, and cyclists at roundabouts.

\subsection{Traffic Conflict Technique}

\subsubsection{Theory and Technique Developments}

As described earlier, the TCT (Perkins \& Harris, 1968) was first introduced in 1968. The theory behind using traffic conflict as a safety measure is based on the fact that accidents result from serious conflicts that could not have been avoided. Hydén (1987) has proposed a severity hierarchy of traffic events that ranged from a base for undisturbed passages to a peak for serious conflicts and accidents. Figure 2.1 shows the hierarchy of interactions between road users.

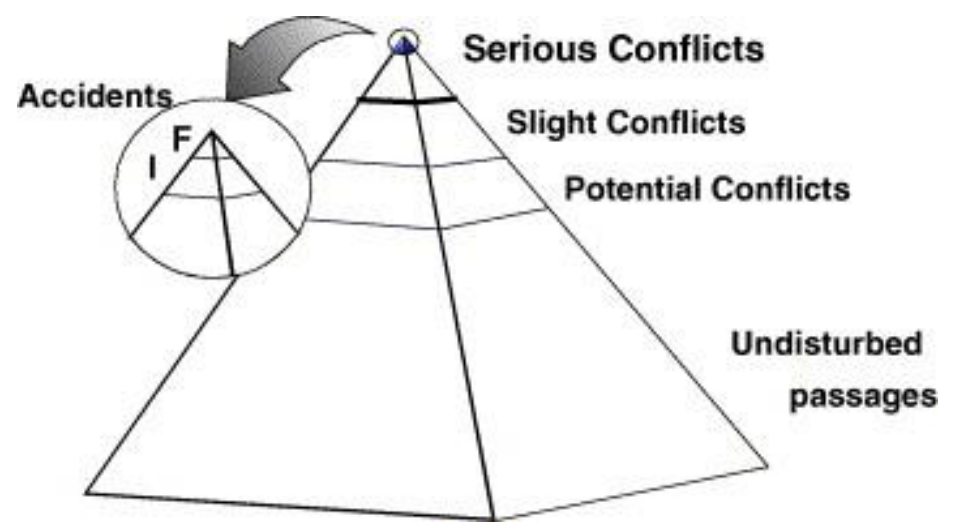

Figure 2.1 The pyramid - the interaction between road users as a continuum of events proposed by Hydén (1987) 
The shape of the safety hierarchy was further discussed by Svensson \& Hydén (2006) who proposed that the shape of the hierarchy should not be pyramidal as was previously suggested, but a diamond shape which is peaked at both sides. The diamond shape was suggested since the accepted gaps and speeds in collision courses were usually normally distributed.

The definition of traffic conflict is based on the presence of an evasive action that changes the movements of the road users (Perkins \& Harris, 1968). However, several researchers (Songchitruksa P., 2004); (Chin \& Quek, 1997)) have noted that an evasive action is not a necessity if accidents are aligned in the same measure with conflicts. Occasionally, it is also difficult to differentiate between evasive and precautionary actions (Songchitruksa P., 2004). This explains why the evasive action has been eliminated from the definition of the conflict as proposed by Amundsen \& Hydén (1977):

"Two or more road users approach each other in space and time to such an extent that a collision is imminent if their movements remain unchanged."

It is important to study the safety hierarchy employing the basic conflict definition as applied to roundabout interactions. Distinguishing between the severity levels of interactions at roundabouts can be more difficult. This issue can be investigated in greater detail through an indepth study of the generated conflicts at the roundabout.

\subsubsection{Objective Conflict's Indicators}

One major shortcoming of the severity hierarchy is that it does not include an objective measure to differentiate between levels of conflict severity. TCT and the estimation of conflict severity were usually studied in a subjective way using time-lapse filming as per the Archer review (2004). Several studies have reviewed the proposed objective indicators in measuring the 
severity of traffic conflicts ( (Songchitruksa P. , 2004); (Ismail K. A., 2010)) and indicators can be summarized as follows:

- Post-encroachment time (PET) is defined as the time interval between two vehicles with crossing paths; as this time interval decreases, the severity of the conflict increases (Songchitruksa P. , 2004). Chin and Quek (1997) have criticized the definition because of the difficulty of differentiating between the unawareness of the drivers and their willingness to take a risk. The extreme value theory approach was proposed by Songchitruksa \& Tarko (2006) who introduced an objective measure in estimating safety by mapping conflicts and collisions together. It was successful in seven out of 12 sites in estimating safety measures using the PET indicator for right-angle collisions, but the extreme value approach did not suggest a proper approach in measuring different levels of severity.

- Time to Accident (TA) indicator has been used by Hydén (1987) with conflicting speed values to link between accidents and conflicts. It is based on estimating the time taken to make evasive actions, and it was concluded that accidents generelly have a 0.5 second higher TA value and a 10-20 kph higher velocity that those found in serious conflicts. This was an important start in quantifing the severity of conflicts, but it also included the subjectivity of how one should estimate the evasive actions of vehicles in order to account for the event as a conflict (Archer, 2004). In addition, the major drawbacks of the TA indicator are that it does not account for the reaction time in estimating the severity value, and requires a collision course to be calculated (Ismail K. A., 2010).

- Time-To-Collision (TTC) was first defined by Hayward (1968) as: 
“...the time that remains until a collision between two vehicles would have occurred if the collision course and speed difference are maintained."

It is considered to be one of the most important objective conflict indicators. It requires that an accurate estimation of the position and velocity of the road user be calculated. One major drawback is that the TTC does not account for the vehicle's velocity in severity calculations, and that it requires a collision course in order to be calculated.

- Gap Time (GT) has been defined as the extrapolated time interval between two vehicles with crossing paths (Archer, 2004). It is similar to the PET indicator but uses extrapolations instead of actual trajectories of road users.

- Deceleration to Safety Time (DST) has been defined by Hupfer (1997) in literature reviews ( (Ismail K. A., 2010); (Songchitruksa P. , 2004)) as an indicator of the severity of a conflict by calculating the required deceleration rate to achieve a non-negative PET value. The DST is used as an indication of the severity of the evasive action made to avoid a collision, and its major drawback is that it assumes an evasive action to be made that is based on extrapolated movements of the road users.

\subsubsection{Time-to-Collision Development}

The TTC indicator has been developed and applied to several studies in literature. Chin \& Quek (1997) have identified several issues in applying the TTC, such as the inconsistency of the traffic conflict definition, the validation method, and the reliability of conflict measurements. Therefore, an improvement in measuring conflicts' severity has been proposed by Saunier \& Sayed (2008) who proposed a probabilistic framework to calculate the probability of a collision based on the previous work of $\mathrm{Hu}$ et al. (2004). This probabilistic framework is based on predicting the movements of the road users, and using previous patterns of the road users. This is 
used to calculate the objective conflict indicator, TTC which was first defined by Hayward (1968).

Further advancement to the movement extrapolation in conflicts analysis has been proposed by Saunier et al. (2010). The authors have refined the probabilistic framework by identifying the potential conflict points with their spatial distribution in estimating the collision probability. Figure 2.2 shows an example of three road users and their potential conflict points. The advanced probabilistic framework is used to calculate the probabilistic TTC indicator.

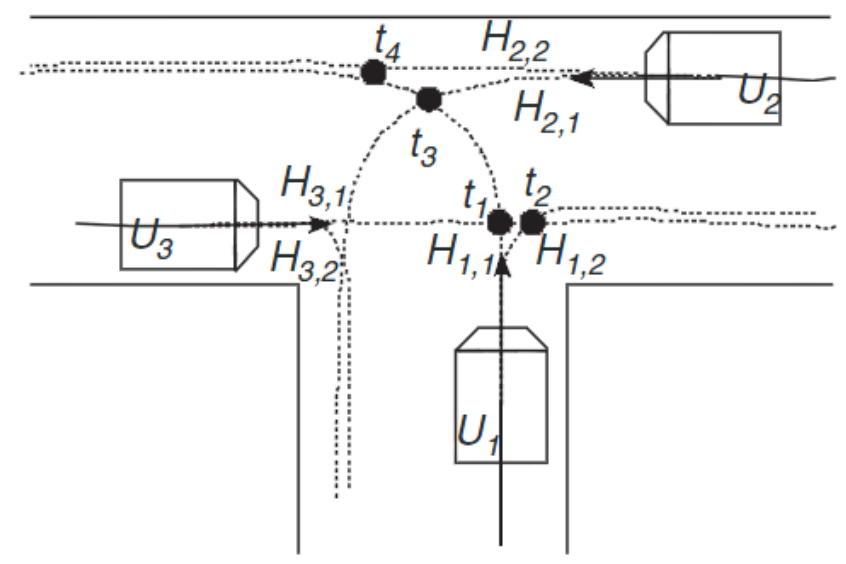

Figure 2.2 Example of three interactions among three road users in four potential conflict points (Saunier, Sayed, \& Ismail, 2010)

The advantage of this framework in calculating the TTC is that it accounts for the probabilities of collision points. The TTC value at a specific time $\left(\mathrm{t}_{0}\right)$ can be calculated by using:

$\operatorname{TTC}\left(t_{0}\right)=\frac{\sum_{1<n<N p} P\left(\operatorname{collision}\left(C P_{n}\right)\right) t_{n}}{\sum_{1<n<N p} P\left(\operatorname{collision}\left(C P_{n}\right)\right)} \quad($ Saunier, Sayed, \& Ismail, 2010)?

Eq. 1 
where $N_{p}$ is the number of potential collision points based on the extrapolations of the vehicles movements, CP is the collision point, and $t_{n}$ is the calculated TTC value for this particular collision point. The probability of a collision is linked with the TTC value as a severity measure using the formula:

Severity $\operatorname{Index}\left(t_{0}\right)=\sum_{1<n<N p} P\left(\operatorname{collision}\left(C P_{n}\right)\right) e^{\frac{-\left(t_{n}-t_{0}\right)^{2}}{2 \sigma^{2}}}$

where $\sigma$ is the normalizing constant, equal to the average reaction time of the user.

The probabilistic TTC indicator has recently been used in several applications related to vehiclevehicle and vehicle-pedestrian interactions ( (Ismail, Sayed, Saunier, \& Lim, 2009); (Autey, Sayed, \& Zaki, 2011)).

\subsubsection{Automation of Video Traffic Tracking and Safety Measures}

One of the earlier studies conducted in traffic tracking and automation is the work of Beymer, McLauchlan, Coifman, \& Malik (1997), where a feature-based tracking system was developed to calculate the traffic parameters from a two-hour video segment. The application was done in a simple through-movement of vehicles in three lanes. An advanced study was conducted by Kamijo, Matsushita, Ikeuchi, and Sakauchi (2000) to monitor traffic and accidents at intersections. Kamijo et al. (2000) developed an algorithm to attempt to overcome video processing issues such as occlusions and cluttered situations.

Kastrinaki, Zervakis, and Kalaitzakis (2003) classified the video techniques based on their input data into three types; feature-driven, area-driven, or model-based. 3-D model-based vehicle tracking was developed by (Hu, Xiao, Xie, \& Tan (2004) to obtain the motion trajectories and to learn the activity patterns of the vehicles. The application was based on the theory of fuzzy sets 
with a developed efficiency and speed of pattern learning, and was a key development in using learned patterns in estimating the probability of a collision. A vision-based approach has been developed by Atev, Massoud, Janardan, and Papanikolopolous (2005) that has been used to predict imminent collisions by extrapolating the positions of the vehicles. The authors have presented several challenges of image processing, such as shadows, occlusions, camera calibrations, and image noise. In addition, an automated video analysis has been developed by Laureshyn \& Ardo (2006) to investigate video processing challenges and to propose better solutions. Their study showed advancement to the estimation of speed and trajectory data for vehicles and pedestrians with limited efficiency, but issues related to diverse object sizes and shapes were still under development. Advancement to the object detection and tracking approach has been proposed by Kim (2008), and is based on dynamic feature grouping with background subtractions. The feature tracking and grouping system was based on comer feature and cluster tracking algorithms, and was applied to video data on vehicles, pedestrians, and cyclists. The system faced similar challenges in trajectory noise and human interference.

A computer vision technique was proposed by Saunier \& Sayed (2006) using a feature-based tracking approach that is based on the feature detection and grouping algorithm. The feature is the smallest distinguishable point in the video frame than can be identified by comparing the object-free background with the current video frame. The quality and quantity of the feature detection process are based on several detection parameters that can be modified across different camera angles and locations. The feature grouping algorithm is based on several grouping parameters such as the maximum and minimum distances between features. Using this automated tracking system, a large-scale analysis of vehicle interactions has been conducted by 
Saunier, Sayed, and Ismail (2010). The authors have proposed a probabilistic framework (Saunier \& Sayed, 2008) in calculating the TTC indicator for road-user interactions.

In summary, existing systems developed for automatic road user detection is sufficient for employment in traffic safety applications. Additional advancement to current tools can be developed when applying them to various traffic situations. To the knowledge of the author, all applications are applied to intersections and normal road segments, and the automatic detection of road users and the identification of safety countermeasures for roundabouts have not yet been implemented.

\subsection{Roundabout Safety}

As previously noted, the automated traffic conflict analysis can be used to effectively evaluate safety at roads, intersections, and roundabouts. To better understand the evaluation results of the roundabout safety, it is important to review previous diagnostic research at roundabouts. Therefore, this section provides a review of previous work on diagnosing and enhancing roundabout safety levels, details the benefits of converting intersections to roundabouts, and addresses the safety issues related to the interactions of vehicles, pedestrians, and cyclists at roundabouts.

\subsubsection{Roundabouts Versus Intersections}

Several studies have demonstrated that converting signalized and stop-controlled intersections to roundabouts has resulted in improved safety performance ( (Schoon \& Minnen, 1993); (Rodegerdts, et al., 2007)). Table 2.1 shows a summary of the before-and-after studies using base safety performance functions (empirical Bayes) for conversions occurred in the United States (Rodegerdts, et al., 2007). Average collision reductions of $35.4 \%$ and $75.8 \%$ in all collisions and injury collisions, respectively, were observed after the conversion to roundabouts. 
Table 2.1 Summary of before and after studies of conversions from intersections to roundabouts (Rodegerdts, et al., 2007)

\begin{tabular}{c|cc|} 
Intersection type before & \multicolumn{2}{c}{ Percent of reduction in } \\
\cline { 2 - 3 } conversion to a roundabout & All collisions & Injury collisions \\
\cline { 2 - 3 } Two-way Stop & $44.2 \%$ & $81.8 \%$ \\
All Signalized & $47.8 \%$ & $77.7 \%$ \\
All Sites & $35.4 \%$ & $75.8 \%$ \\
\end{tabular}

Installing roundabouts has resulted in improved safety performance at intersections. However, an important issue which requires investigation is the effect of the conversions to roundabouts on the safety of vulnerable road users (Rodegerdts, et al., 2007). This issue will be discussed in the following section.

\subsubsection{Safety of Vehicles}

As the automated traffic conflict analysis can effectively be used to evaluate safety of vehicles at roundabouts, it is important to understand the various evaluation methodologies of vehicles safety. This section provides a review of previous work done on the vehicle collision contributing factors and the vehicle collision patterns at roundabouts.

\subsubsection{Vehicle collision contributing factors}

Several studies attempted to identify collision contributing factors and collision patterns at roundabouts. Montella (2011) explored the interdependences between the collision contributory factors themselves, and between the contributory factors and different types of collisions. His research has showed that $60 \%$ of collisions are associated with at least one geometric design factor. Figure 2.3 presents the main roundabout geometric design parameters including the radius of deflection, the deviation angle, and entry and exit angles. Contributory factors were classified 
as major and minor factors based on their contribution to collisions, and this decision was based on the opinions of safety experts which may include some subjectivity and uncertainty. The most important geometric design factors identified were the excessive radius of deflection and the low angle of deviation. As Montella (2011) mentioned in his literature review, several studies ((SETRA, 1998); ( Elvik et al., 2009)) have shown that traveling vehicles should not follow a path in the roundabout with a radius of deflection of more than 100 meters (the UK standard suggests a threshold of 60 meters). It also has been shown that the deviation angle parameter was considered a contributing factor for a major collision if the angle was less than 30 degrees.

Due to inadequate yield lines and symbols, markings have also been identified as a contributory factor for more than $50 \%$ of collisions, and signs have been identified as a minor contributing factor in more than $30 \%$ of collisions.

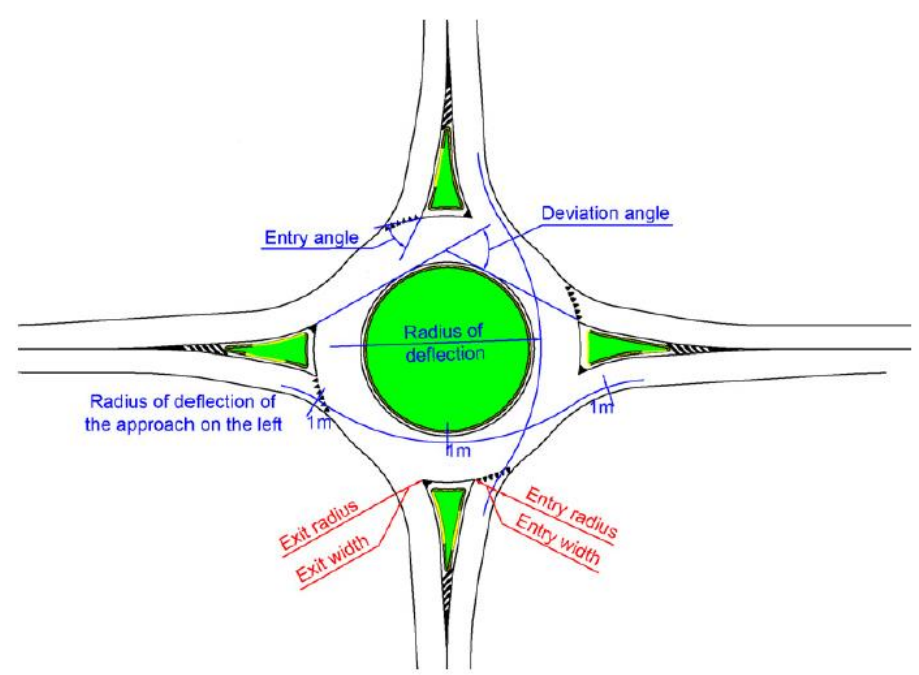

Figure 2.3 The main roundabout geometric design parameters (Montella A. , 2011)

It is important to note that the association between factors in this study do not imply causality, but rather attempt to associate collisions with contributing factors. As shown in Figure 2.4, it is important to differentiate between the cause (e.g.: the high entering speed of the vehicle) and 
contributing factors (e.g.: the low angle of deviation). If a collision occurred with a circulating vehicle, the cause would be the high entering speed (failure to yield), and the effect (the result or impact) is the collision.

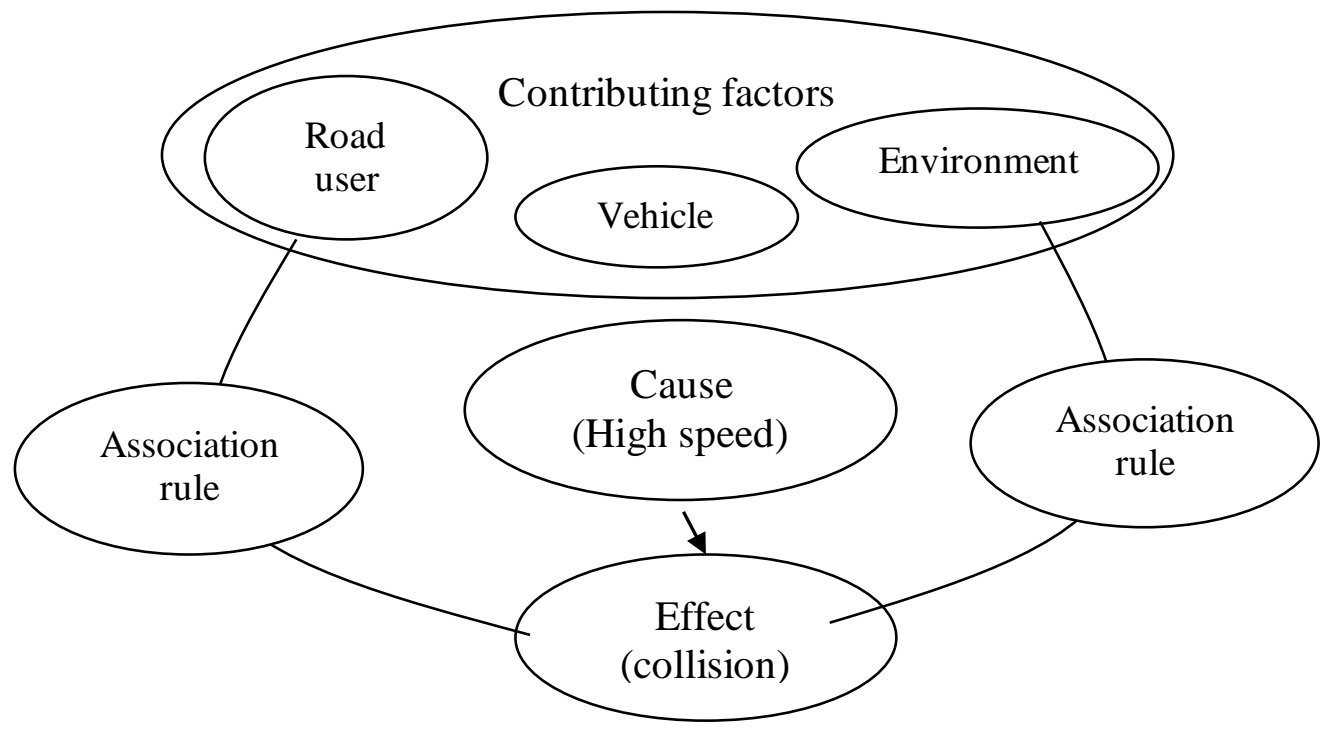

Figure 2.4 Difference between contributing factors and collision causes

The association methodology was applied between "collision contributing factors" and collisions. In traffic conflict analysis, the utilization of association methodology is different when applied in traffic conflict analyses since the result is only a conflict and not an actual collision. However, conflict analysis gives more insight for answering the question: "Why did the road users fail to cope with the environment?" The term "conflict contributing factor" can be used in association methodology for generated conflicts in the roundabout.

\subsubsection{Vehicle collisions patterns}

A study of Maryland roundabouts (Mandavilli, McCartt, \& Retting, 2009) was conducted to develop a typology of collisions at roundabouts. It was found that $50 \%$ of collisions were singlevehicle run-off-road collisions, rear-end and sideswipe collisions represented $34 \%$ and $24 \%$ of 
collisions in roundabouts, respectively. Entering-circulating collisions represented $14 \%$ and $17 \%$ of collisions in single and double-lane roundabouts, respectively.

Zheng, Qin, \& Noyce (2010) conducted a comprehensive analysis of collision patterns at Wisconsin roundabouts, with $40 \%$ of the collisions to be entering-circulating collisions at singlelane roundabouts, and run-off-road and rear-end collisions to be $32 \%$ and $23 \%$ of collisions, respectively, at single-lane roundabouts.

Collision types cannot always be correlated with conflict types. For example, run-off-road is a collision type that cannot be observed in conflicts analyses; however, other types of collisions can be correlated with conflict types such as rear-end, entering-circulating, and sideswipe collisions.

\subsubsection{Safety of Pedestrians}

As the automated traffic conflict analysis can be used to effectively evaluate safety of pedestrians at roundabouts, it is important to understand the evaluation methodologies of the pedestrians' safety. This section provides a review of previous work done on the pedestrian crossing behaviour, crosswalk design, signalized crosswalks, and vehicle-pedestrian collision patterns.

\subsubsection{Crossing behaviour of pedestrian}

One important factor that affects the safety of pedestrians at intersections and roundabouts is their crossing behaviour. Waiting time has been used as an indicator of crossing behaviour for pedestrians at a modern roundabout (Ashmead, Guth, Wall, Long, \& Ponchillia, 2005), and is calculated from the time of arrival at the crossing location, up to the time the crossing began. Gap durations have also been used to measure ability of pedestrians to cross. In the aforementioned study, sighted pedestrians had average accepted gaps of 12 seconds. 
Schroeder, Rouphail, \& Hughes (2009) used a different methodology than waiting time and gap duration in measuring the safety performance of pedestrians. The authors used the probability of vehicle yielding (yielding availability) and the probability of yields that resulted in a pedestrian crossing (yielding utilizations). They applied the methodology on a single-lane roundabout. The probability of yielding was higher for the entry than the exit lane with values of $41.5 \%$ and $32.8 \%$, respectively. In addition to yielding availability, yielding utilization in the entry lane was less than that of the exit lanes with $83 \%$ and $87.8 \%$, respectively. The same concept is used in calculating the probability of crossable gaps and the probability of crossable gaps that resulted in pedestrians crossing. The probability of a crossable gap's occurring was slightly higher for the entry than the exit lane with $53.5 \%$ and $50.2 \%$, respectively. Other than crossable gap availability, gap utilization at the entry lane was less than that of the exit lanes with $52 \%$ and $63.6 \%$, respectively. Provided that the probability of crossable gaps is higher for entry lanes, pedestrians are more able to utilize the gaps at exit lanes.

In the previous studies, performance measures are normally calculated using a controlled experimental environment to test pedestrian behaviour. Therefore, it can be implied that the performance measures may be biased because of the skills and controlled crossing behaviour of the participants. This bias could be reduced by testing real-time pedestrian crossing behaviour in an automated traffic conflicts analysis.

Furthermore, there are different methodologies utilized in analyzing the behaviour of the pedestrians. Rodegerdts et al. (2007) noted that if pedestrians are crossing and interacting with vehicles, behaviour can be categorized as: 
- Normal: when a pedestrian crosses at a normal walking speed and none of the following behaviours is observed.

- Hesitates: when a pedestrian hesitates about the decision to cross while he or she is still on the curb or splitter island.

- Retreats: when a pedestrian has begun to cross and then decided to retreat to the curb or the splitter island.

- Runs: when a pedestrian runs because of an approaching vehicle.

These categories assist in demonstrating how pedestrians interact with vehicles and how safe the crosswalk is designed.

Pedestrian behaviour can be also demonstrated by understanding the yielding behaviour of interacting vehicles. Rodegerdts et al. (2007) noted that yielding behaviour can be categorized as follows:

- Active yield: the motorist slows or stops only because of a crossing or a waiting pedestrian.

- Passive yield: the motorist slows or stops not because of a crossing or a waiting pedestrian but due to another reason, such as a queue of vehicles waiting to enter or exit the roundabout or already waiting for a previous pedestrian.

- Did not yield: the motorist did not yield for the crossing or waiting pedestrian.

In literature, most pedestrian behaviour analyses are based on manual video reviews and human observations. The automated traffic conflict analysis is invaluable in automatically producing sets of videos of these interactions for further analyses, which reduces the time and resources needed to perform pedestrians' analyses. 


\subsubsection{Pedestrian cross walk design}

Crosswalk design significantly affects the safety performance of pedestrians crossing at roundabouts. Crosswalk design includes the location, and the angle of crosswalks and splitter island between entry and exit lanes.

In the NCHRP report (2010), the location of crosswalks is suggested to be at least six meters (one vehicle length) before the circulating roadway. It is suggested to double or triple this distance if required in some specific cases of crosswalk design.

The trade-off between yielding behaviour and the deflection angle of the roundabout has also been investigated and has been found that increasing the deflection angle of the entry leg will reduce the vehicle speed and increase the active yielding of the pedestrian crossing. However, high deflection angles will lead to a higher vehicular speed while exiting the roundabout. This method of alignment is called the offset approach alignment and is shown in Figure 2.5.

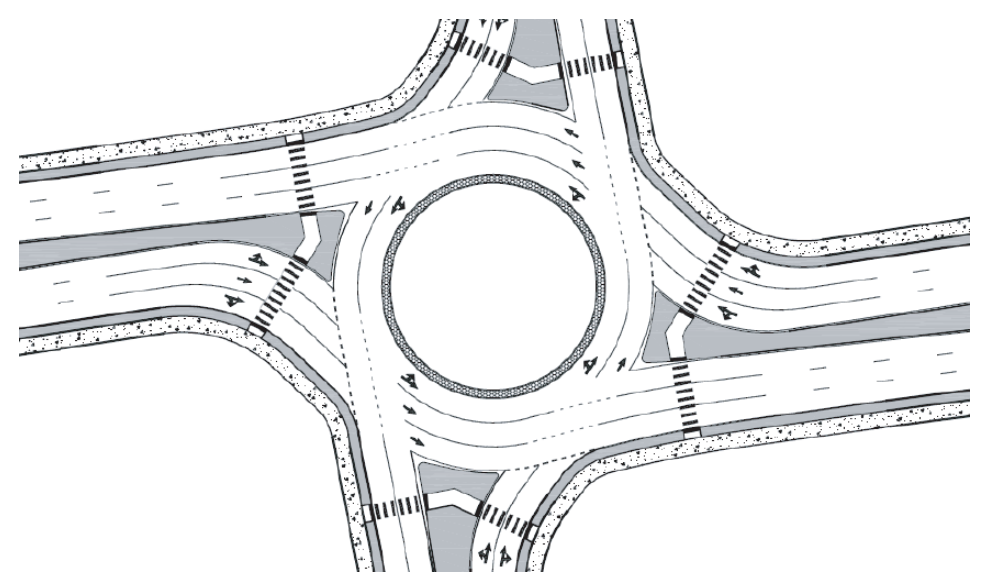

Figure 2.5 Offset approach alignment to increase deflection angle and reduce vehicle entry speed

Baranowski (2005) suggested that an offset between the entry and exit crosswalks as shown in Figure 2.6, will help in avoiding the pedestrian "see through" action and should achieve a twostaged crossing behaviour. 


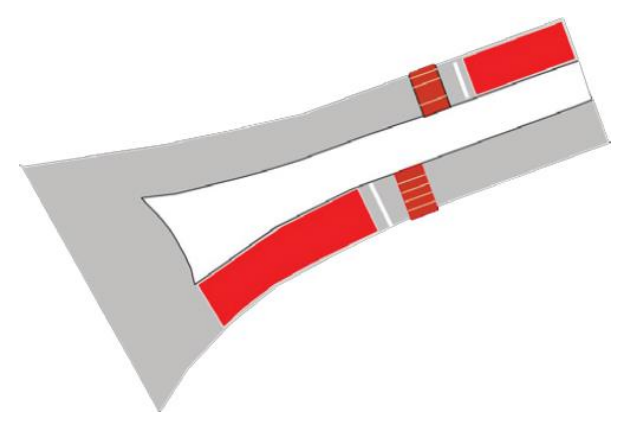

Figure 2.6 Offset in crosswalk between entry and exit legs (Baranowski, 2005)

Finding the crosswalk is a critical issue for pedestrians. It is suggested that proper landscaping design can help locate the crosswalk ( National Cooperative Highway Research Program (NCHRP), 2010). Lenters (2005) discussed the use of fencing to restrict pedestrian crossing in the specified location. However, fencing may not always be practical due to the snow cleaning techniques during the winter. It is suggested that landscaping can perform the same function unless fencing is mandatory.

Four types of crosswalk surfaces have been discussed by Skene et al. (2010). A crosswalk usually is constructed on the same level of the roadway, and with a tactile surface on the approach road way (as rumble strips). In some locations, crosswalks are constructed with a speed hump, a raised pavement, or a raised pavement with a tactile surface. All four types affect how a pedestrian locates the crosswalk and how a driver interacts with a pedestrian's crossing. Skene, et al. (2010) recommends using the tactile approach or rumble strips, as they assist the pedestrian in locating the crosswalk and reduces the impact on vehicle movement.

\subsubsection{Signalized cross walks}

Roundabouts generally differ from regular intersections in that they do not have any traffic control devices. However, Baranowski (2005) suggests that crosswalk signals at roundabouts can 
be used in some cases, and the main objective of a signalized crosswalk is to increase the safety of pedestrians. The crosswalk signal can be applied in two different ways:

- A standard traffic signal with a green-yellow-red signal activated by a pedestrian pushbutton

- A flashing yellow beacon or in-pavement flashers or the combination of both also activated by a pedestrian pushbutton.

These two options are warranted by the Manual on Uniform Traffic Control Devices (MUTCD) (FHWA, 2009) if there is a high number of pedestrians crossing at the roundabout. Several studies have recommended other criteria levels to satisfy the "high" number of pedestrians. For example, MUTCD recommends a pedestrian crossing signal if traffic volume is so heavy that pedestrians would experience an excessive delay to cross. Figure 2.7 shows the threshold charts used in the MUTCD (FHWA, 2009) to apply the pedestrian crossing signal. 

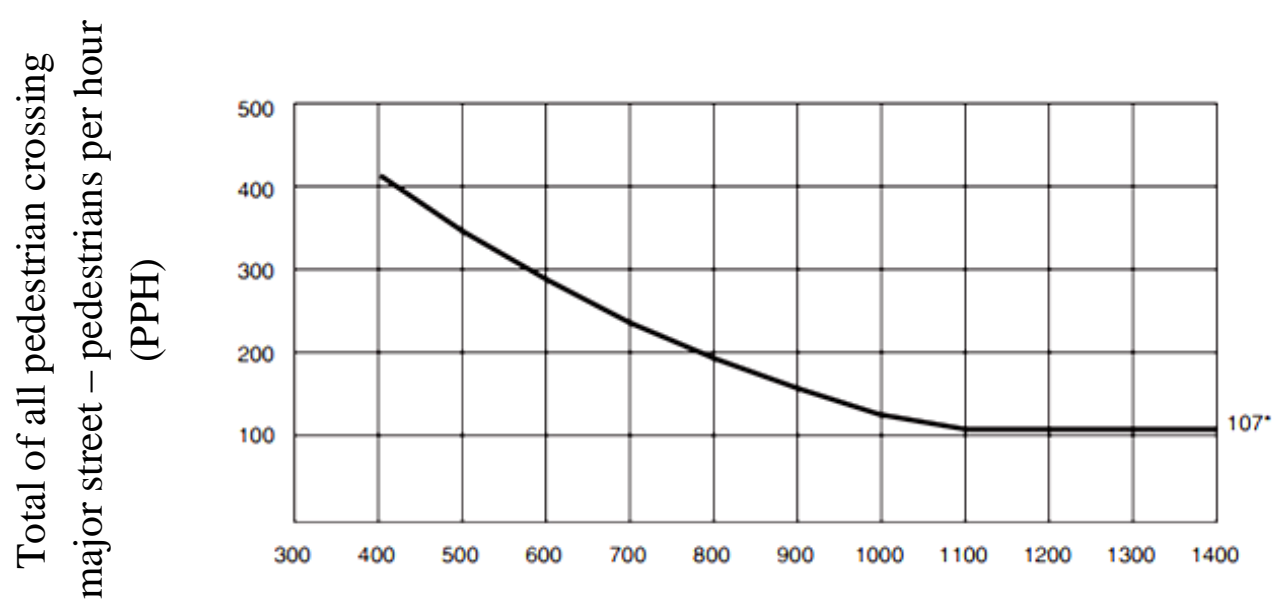

(Major street - total of both approaches - vehicles per hour (VPH) * Note: 107 pph applies as the lower threshold volume

(a)
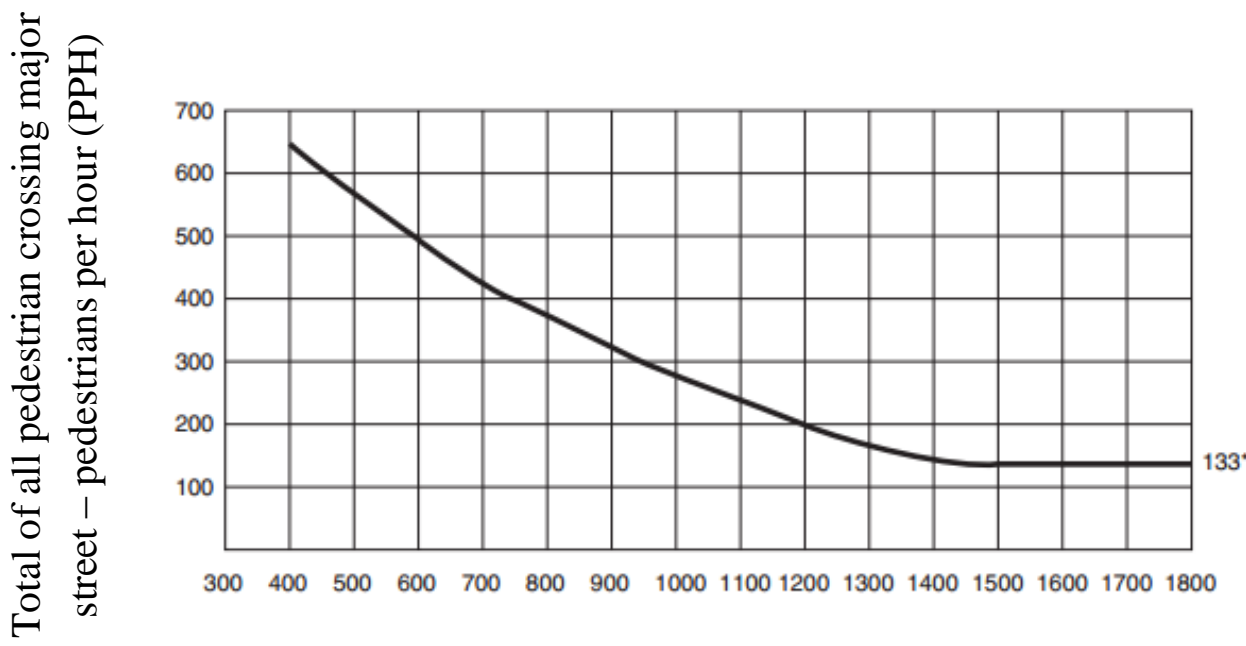

(Major street - total of both approaches - vehicles per hour (VPH) * Note: 133 pph applies as the lower threshold volume

(b)

Figure 2.7 Threshold chart to apply pedestrian crossing signal for (a) four-hour volume, and (b) one-hour volume (FHWA, 2009)

Baranowski (2005) has referenced literature in Britain, as they warrant a pedestrian crosswalk signal according to the following criteria: 
$\mathrm{PV}^{2}>10^{8}$,

where $\mathrm{p}$ is the pedestrian volume per hour, and $\mathrm{V}$ is the entering vehicle volume average over the four peak hours.

Lenters (2005) discourages the use of pedestrian crossing warning signs that warn drivers there is a pedestrian crossing ahead. He notes that warning signs may confuse road users because the right-of-way can differ from one jurisdiction to another, and some highway codes preserve the right-of-way for drivers, so this warning may confuse their expectations. Otherwise, if the common use of the jurisdiction is to give the right of way to the pedestrian, the warning sign may improve the safety of the crossing.

Lu, Guan, \& Noyce (2010) have proposed an intelligent management system to improve the accessibility of pedestrian in roundabouts. Fuzzy logic control (FLC) was used in the signal timing design, and proposed that the distant signal pedestrian crossing (Figure 2.8) can improve the roundabout accessibility, safety, and efficiency.

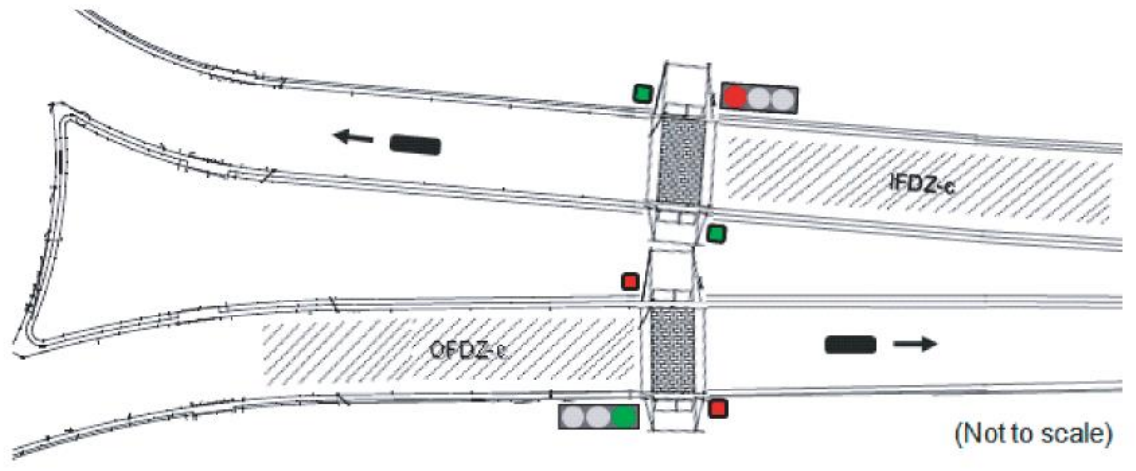

Figure 2.8 The distant signal pedestrian crossing layout (Lu, Guan, \& Noyce, 2010) 


\subsubsection{Vehicle-Pedestrian collisions patterns}

Roundabouts generally decrease the conflict points between vehicles, and they also decrease pedestrian collisions. For example, in the Netherlands, the conversion of intersections into roundabouts has reduced pedestrian collisions by around 73\% (Schoon \& Minnen, 1993). However, the absence of traffic control devices at roundabouts and the increase of the popularity of roundabouts raise the significance of understanding vehicle-pedestrian collision patterns. Mandavilli, McCartt, \& Retting (2009) have conducted an analysis on collision patterns at Maryland roundabouts for a period of at least two years. They determined that in double-lane roundabouts, $4 \%$ of collisions are pedestrian-vehicle or cyclist-vehicle collisions and that Singlelane roundabouts did not have any pedestrian-vehicle collisions. Zheng, Qin, \& Noyce (2010) conducted a comprehensive analysis for collision patterns in Wisconsin roundabouts, and found that all collisions were vehicle-vehicle collision and no vehicle-pedestrian collisions were recorded in the collision database for approximately seven years (March 2001 to August 2009). Montella (2011) studied the contributing factors for collisions occurring in 15 roundabouts in Italy for five years (2003 to 2008). His data revealed that $2.6 \%$ and $1.1 \%$ of collisions were vehicle-pedestrian collisions that occurred in exit and entry lanes, respectively. This low ratio of vehicle-pedestrian collisions is not necessarily an indication of a safe situation, because unsafe interactions by pedestrians may contribute to the vehicle-vehicle collisions but without reporting them in the collisions records. Pedestrian demand on the roundabout may also be lower than expected because of the safety perception of the crossing. Further investigation is required to quantify, assure and improve pedestrian safety at roundabouts. 


\subsubsection{Safety of Cyclists}

As the automated traffic conflict analysis can effectively be used to evaluate safety of cyclists at roundabouts, it is important to understand the evaluation methodologies of their safety. This section provides a review of previous work done on the behaviour of cyclists, and the different roundabout designs to accommodate them.

\subsubsection{Cyclist behaviour}

The safety of cyclists at roundabouts has always been a concern, especially with the growing rate of roundabout installations. Roundabouts generally decrease the conflict points between vehicles, but several studies have shown that cyclists collisions at roundabouts increased or need further investigation ( (Daniels, Nuyts, \& Wets, 2007); (Brown, 1995)). In this section, a close literature review of cyclist safety is conducted focusing on cyclist behaviour, conflicts and collisions.

Harkey \& Carter (2006) have conducted an observational study for the behaviour of cyclists, which has been classified into three categories based on their location as they enter, exit or traverse the roundabout. The position of cyclists is classified as on the sidewalks, shoulder, bike lane, or edge of a travel lane. The data used was collected for 690 cyclists' events at 14 approaches at seven roundabouts throughout the United States. It was found that $54 \%, 28 \%$, and $18 \%$ of cyclists rode on the edge of the lane (or bike lane or shoulder), possessed a lane, or rode on the sidewalks, respectively. The interactions of cyclists were also classified based on their behaviour as follows:

- Behaves normally without any change in their movement because of an approaching vehicle,

- Hesitates before interacting with a vehicle, or waits until a vehicle cleared the area, 
- Hesitates while interacting with a vehicle,

- Retreats after interacting with a vehicle,

- Swerves to avoid an approach vehicle.

Harkey \& Carter (2006) also classified cyclists' behaviour while entering the roundabout. 70\%, of entering cyclists have entered circulating lanes without waiting or stopping. $11 \%$ of entering cyclists have waited before entering circulating lanes, while $19 \%$ of them have used the crosswalks. $97 \%$ of entering cyclists have not interacted with circulating vehicles. There were five interactions $(3 \%)$ between entering cyclists and vehicles and they have been as follows:

- A vehicle slowed for a waiting cyclist.

- A vehicle yielded for a waiting cyclist (which is considered an improper negotiation).

- A vehicle swerved to avoid a cyclist

- A vehicle pass by the right side of a cyclist

- A vehicle passed by the left side of a cyclist

In the aforementioned study, $62 \%$ and $77 \%$ of crossing cyclists have not encountered any interaction with vehicles at entry and exit lanes, respectively. 5\% and $11 \%$ of vehicles have slowed for waiting cyclists at entry and exit lanes, respectively. $14 \%$ and $11 \%$ of vehicles have slowed for crossing cyclists at entry and exit lanes, respectively. $20 \%$ and $1 \%$ of vehicles were already stopping for other reasons at entry and exit lanes, respectively.

Sakshaug, Laureshyn, Svensson, \& Hyden (2010) conducted a study about cyclist safety at roundabouts. Using five days of video recorded (45 hours) in two roundabouts, it was found that cyclist-vehicle interactions occurred between entering vehicles and circulating cyclists. 4 out of 10 cyclist-vehicle conflicts occurred due to entering vehicles not yielding for circulating cyclists. 
3 out of 10 cyclist-vehicle conflicts occurred due to cyclists not yielding for circulating vehicles. Cyclists' behaviour was also analyzed based on yielding and reaction behaviours. For example, entering vehicles have yielded for circulating cyclists in $96 \%$ of interactions, while circulating cyclists has yielded to entering vehicles in $4 \%$ of interactions. $92 \%$ of successful yielding for circulating cyclists led to keep them circulating without any change in their behaviour, while $8 \%$ resulted in some behaviour adjustment for circulating cyclists. Cyclists' behaviour has also been classified in their interactions based on five actions; no speed change, get off the bike, adjust speed, stop, and stand still. Parallel interactions between cyclists and vehicles have been classified based on two actions; moves in parallel, and stays behind.

Cyclists behaving as pedestrians were also analyzed based on the motorist yielding behaviour. In research performed by Sakshaug et al. (2010), entering vehicles and exiting vehicles have generally yielded to crossing cyclists in $69 \%$ and $61 \%$ of the interactions. Entering vehicles yielded to crossing cyclists from the splitter island in $87 \%$ of the interactions, while entering vehicles yielded to crossing cyclists from the side curb in $64 \%$ of the interactions. Exiting vehicles have yielded to crossing cyclists from the splitter island in $75 \%$ of the interactions, while exiting vehicles has yielded to crossing cyclists from the side curb in 55\% of the interactions.

Generally, the behaviour of cyclists has been analyzed in the literature to investigate safety issues. Therefore, the methodologies used are usually subjective and include considerable manual (human) intervention. Further research is required to understand the behaviour of the cyclist in a quantitative and objective manner. The use of TCT can be an important step to quantitatively evaluate the severities of the interactions of cyclists. 


\subsubsection{Cyclists in roundabout designs}

Daniels et al. (2009) conducted a study on the cycle facilities design on roundabouts. The authors have classified the types of cycle facilities; mixed traffic, cycle lane within the roundabout, separate cycle path, or grade-separated cycle paths. The four basic types are shown in Figure 2.9.

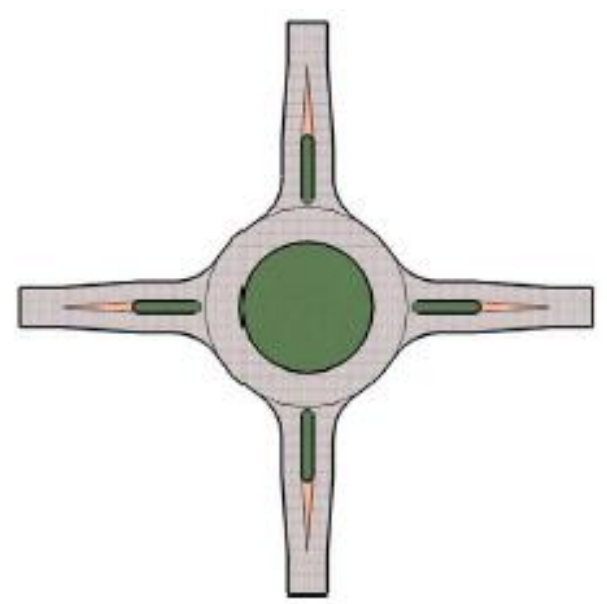

(a)

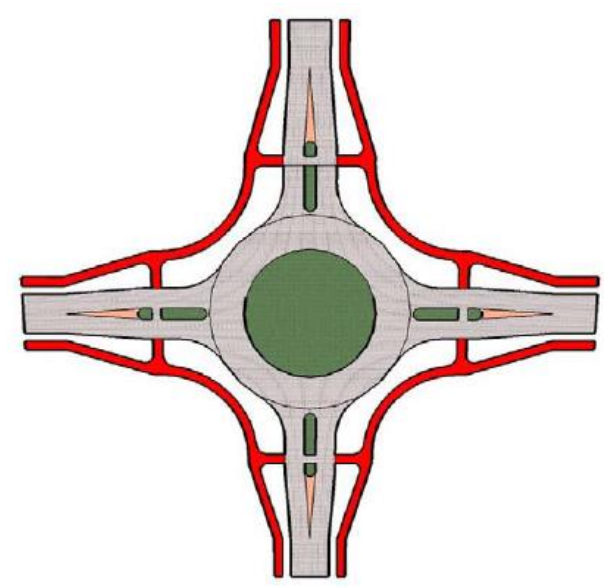

(c)

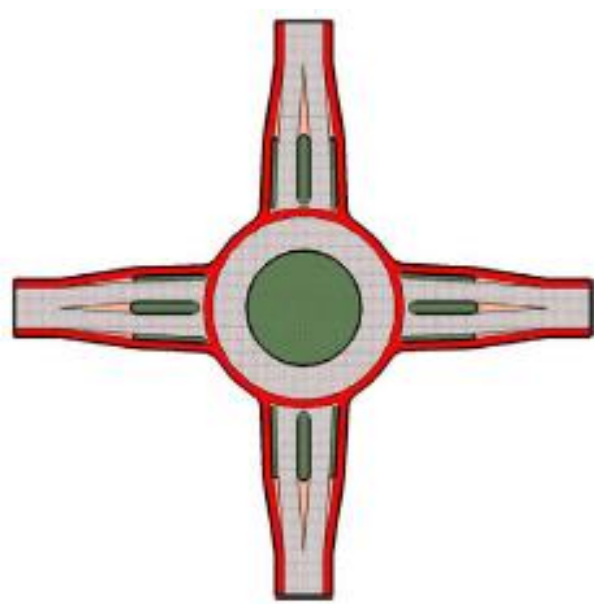

(b)

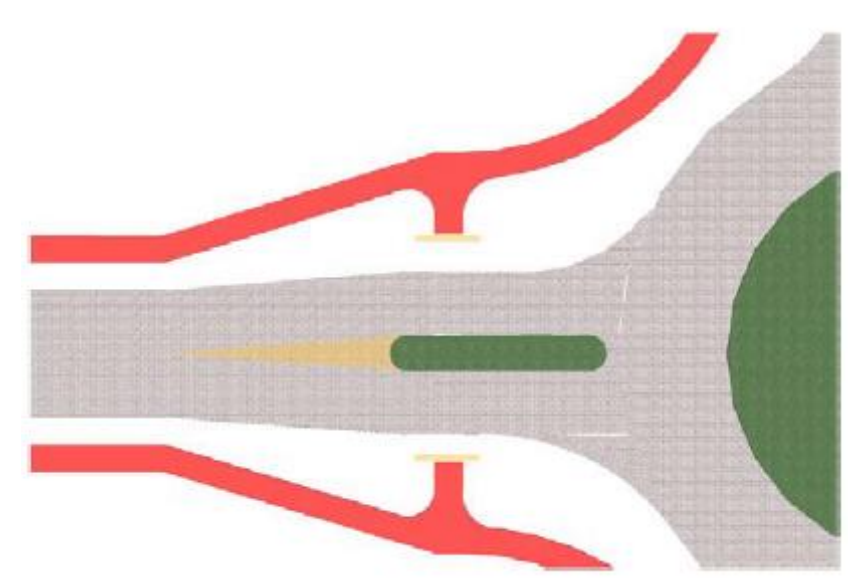

(d)

Figure 2.9 Four basic types of cycle facilities: (a) mixed traffic; (b) cycle lane within the roundabout; (c) separate cycle path; and (d) grade-separated cycle paths (Daniels, Brijs, Nuyts, \& Wets, 2009). 
The aforementioned study has shown that all types of cycle facilities designs have resulted in an increase of the severe injury cyclist collisions at roundabouts. It was also shown that separate cycle path design may have higher impact in severe injury cyclist collisions.

Sakshaug et al. (2010) conducted their research in two types of cycle facilities design; the separated roundabout (which is similar to separate cycle path type in the previous study), and the integrated roundabout (which is similar to the traffic mix type in the previous study). Both types of roundabouts are shown in Figure 2.10.

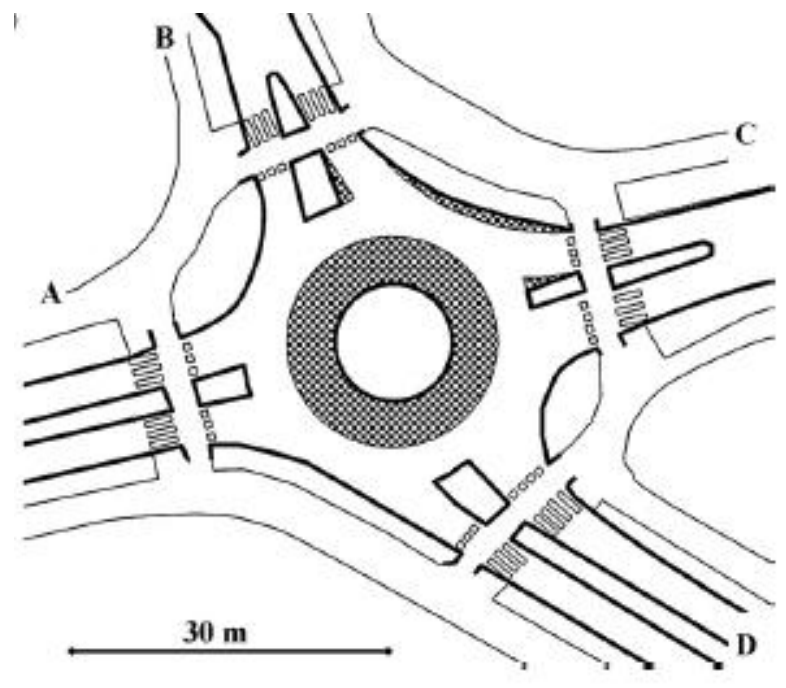

(a)

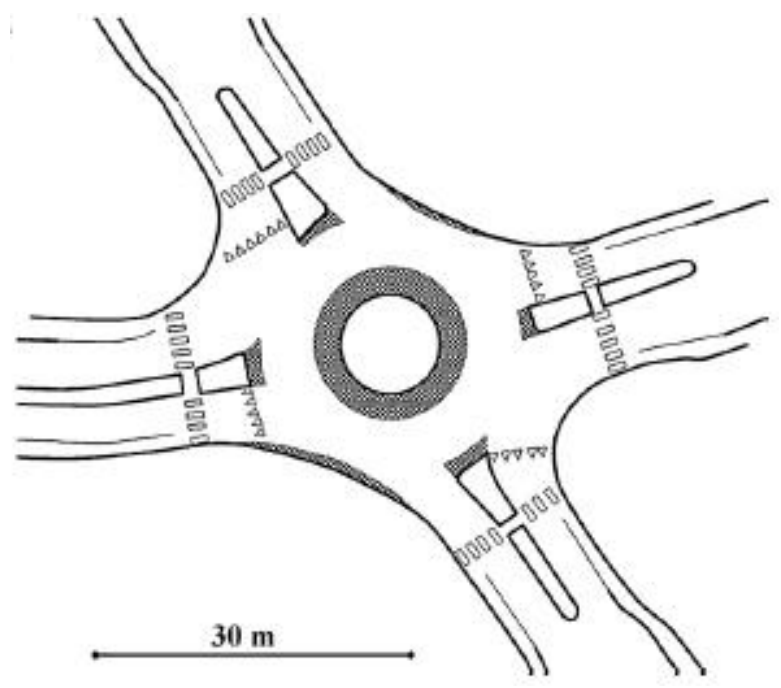

(b)

Figure 2.10 Two types of cycle facilities: (a) a separated roundabout (with a separate cycle path); and (b) an integrated roundabout (mixed traffic).

The authors did not indicate which cycle design facilities would be the safest, but their results were in agreement with previous research that indicated a separated cycle crossing design appears to be safer than other designs. On the other hand, a previous research by Daniels et al. (2009) showed that a separate cycle path design seems to result in more severe injury collisions. 
On this basis, therefore, the current literature has not confirmed the effect of cycle path designs on safety performance. 


\section{Methodology and Data Collection}

In this chapter, the methodology used in the safety diagnosis of the roundabout (located next to UBC campus) will be described, and will include the safety diagnosis process steps and the data collection. The diagnosis process steps describe how the safety of the roundabout is evaluated, and how the TCT is applied. This chapter will also present the data collection and camera calibration process, as well as describing the image processing and the events generation using the UBC automated safety analysis tool.

\subsection{The Safety Diagnosis Process}

To better understand the benefits of using the automated TCT in the safety diagnosis, it is important to define how this conflict information can be used in a systematic way to diagnose the safety of the location. The traffic safety diagnosis and operational analysis of the roundabout consist of the following steps (Sayed, deLeur, \& Sawalha, 2004):

1. In-office analysis:

- Preliminary review of the roundabout operation by analyzing the vehicle traffic volume, and vulnerable road user volume to find the general safety issues in the roundabout.

2. On-site observation and conflict generation:

- Visit the roundabout site and drive through it to examine the physical features of the location and general traffic operation.

- Record the videos for the roundabout and implement the TCT, using the UBC automated safety tool, to find the conflicts occurring at the roundabout. 
- Review conflict points, conflict rates, and videos to identify over-represented clusters of particular conflicts.

3. Identify possible causes of traffic conflicts:

- Utilize the information from step 1 and step 2 to identify the possible causes of these conflicts types and other safety issues.

4. Identify possible countermeasures to improve the safety of the roundabout:

- Generate a list of possible engineering countermeasures and rank them based on their effectiveness.

Step 1, 2 and 3 will be discussed in the Results and Discussion section of this thesis. Step 4 will be discussed in the Recommendations section.

\subsection{Data Collection and Implementation}

\subsubsection{Video Recording and Encoding}

The roundabout is located at $16^{\text {th }}$ avenue and Wesbroock Mall in the city of Vancouver, British Columbia, Canada. Five cameras have been used and mounted on a nearby building. Figure 3.1 shows the focus area for each camera scene. Cameras have been positioned to cover most areas of the roundabout, except the shaded parts. Furthermore, view obstructions limited the ability to mount a camera to capture the crosswalk at approach D, as shown in Figure 3.1. 


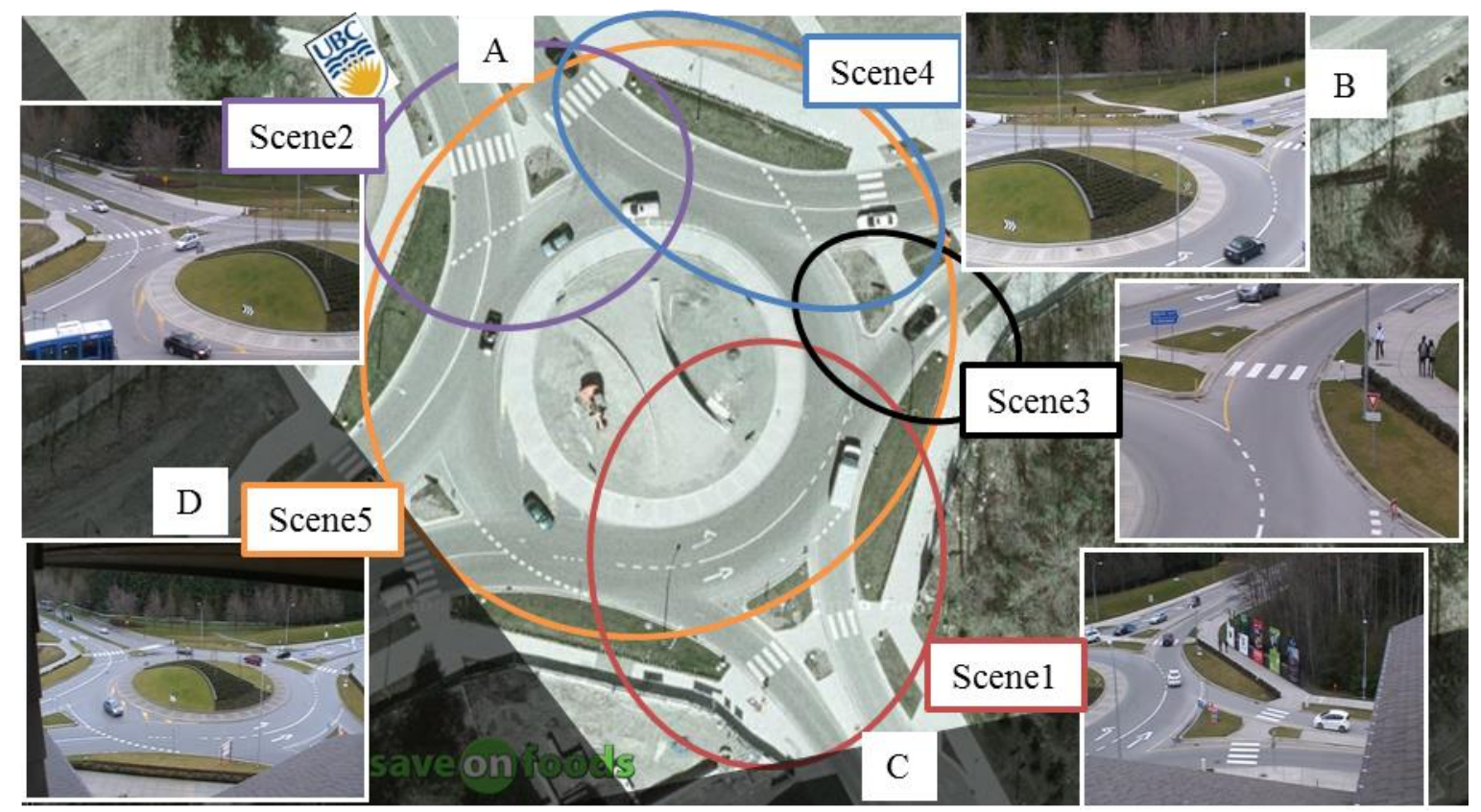

Figure 3.1 The focus area of the five cameras scenes

Table 3.1 shows the details of the video recording. The video data was collected on 16 and 17 of March of year 2011 (Wednesday and Thursday).

Table 3.1 Video recording periods and details for the five cameras

\begin{tabular}{c|ccc|ccc|} 
Scene & \multicolumn{2}{|c|}{ Day 1: Wednesday 16-March-2011 } & \multicolumn{2}{c}{ Day 2: Thursday 17-March-2011 } \\
\hline & Start time & End time & $\begin{array}{c}\text { Total duration } \\
\text { (hr:min) }\end{array}$ & Start time & End time & $\begin{array}{c}\text { Total duration } \\
\text { (hr:min) }\end{array}$ \\
1 & $09: 08 \mathrm{AM}$ & $08: 27 \mathrm{PM}$ & $11: 19$ & $09: 16 \mathrm{AM}$ & $08: 35 \mathrm{PM}$ & $11: 19$ \\
2 & $09: 08 \mathrm{AM}$ & $08: 55 \mathrm{PM}$ & $11: 47$ & $09: 16 \mathrm{AM}$ & $08: 59 \mathrm{PM}$ & $11: 43$ \\
3 & $09: 08 \mathrm{AM}$ & $08: 43 \mathrm{PM}$ & $11: 35$ & $09: 16 \mathrm{AM}$ & $08: 54 \mathrm{PM}$ & $11: 38$ \\
4 & $09: 08 \mathrm{AM}$ & $08: 50 \mathrm{PM}$ & $11: 42$ & $09: 16 \mathrm{AM}$ & $09: 00 \mathrm{PM}$ & $11: 44$ \\
5 & $09: 08 \mathrm{AM}$ & $09: 00 \mathrm{PM}$ & $11: 52$ & $09: 16 \mathrm{AM}$ & $09: 00 \mathrm{PM}$ & $11: 44$ \\
\hline
\end{tabular}


Video encoding was then performed to convert the videos to the Audio Video Interleave (AVI) format used by the UBC automated video analysis tool. The process is also performed to cut the 11-hour video for each camera into 1-hour segments. This also helps to synchronize all videos from the different camera angles to have identical start time. The output of this stage is a set of one-hour AVI video components with date and time added at the corner of the video. Table 3.2 presents the list of video components details for scene one in day one.

Table 3.2 List of video components details for scene 1 in day 1

Scene Day Components Start time End time Duration (hr:min)

\begin{tabular}{l|ccccc|}
\hline 1 & 1 & 1 & $9: 08 \mathrm{AM}$ & 10:00 AM & $0: 52$ \\
1 & 1 & 2 & $10: 00 \mathrm{AM}$ & $11: 00 \mathrm{AM}$ & $1: 00$ \\
1 & 1 & 3 & $11: 00 \mathrm{AM}$ & $12: 00 \mathrm{PM}$ & $1: 00$ \\
1 & 1 & 4 & $12: 00 \mathrm{PM}$ & $1: 00 \mathrm{PM}$ & $1: 00$ \\
1 & 1 & 5 & $1: 00 \mathrm{PM}$ & $2: 00 \mathrm{PM}$ & $1: 00$ \\
1 & 1 & 6 & $2: 00 \mathrm{PM}$ & $3: 00 \mathrm{PM}$ & $1: 00$ \\
1 & 1 & 7 & $3: 00 \mathrm{PM}$ & $4: 00 \mathrm{PM}$ & $1: 00$ \\
1 & 1 & 8 & $4: 00 \mathrm{PM}$ & $5: 00 \mathrm{PM}$ & $1: 00$ \\
1 & 1 & 9 & $5: 00 \mathrm{PM}$ & $6: 00 \mathrm{PM}$ & $1: 00$ \\
1 & 1 & 10 & $6: 00 \mathrm{PM}$ & $7: 00 \mathrm{PM}$ & $1: 00$ \\
1 & 1 & 11 & $7: 00 \mathrm{PM}$ & $8: 00 \mathrm{PM}$ & $1: 00$ \\
1 & 1 & 12 & $8: 00 \mathrm{PM}$ & $8: 27 \mathrm{PM}$ & $0: 27$ \\
\cline { 2 - 5 }
\end{tabular}

\subsubsection{Camera Calibration}

Camera calibration is the mapping process used to recover the positions of the road users, from the plain image coordinates to the world coordinates. This process has been developed and applied in several previous studies (Ismail, Sayed, Saunier, \& Lim, 2009). The camera 
calibration process requires user defined inputs to calculate a matrix called the homography matrix. The homography matrix includes extrinsic and intrinsic parameters which are used by the UBC automated traffic safety tool to recover the world coordinates of the image.

The camera calibration process was applied to the five camera scenes for both days. Figure 3.2 shows an example of the feature corresponding points and distance lines that are used to match the video image and the world image for scene one.

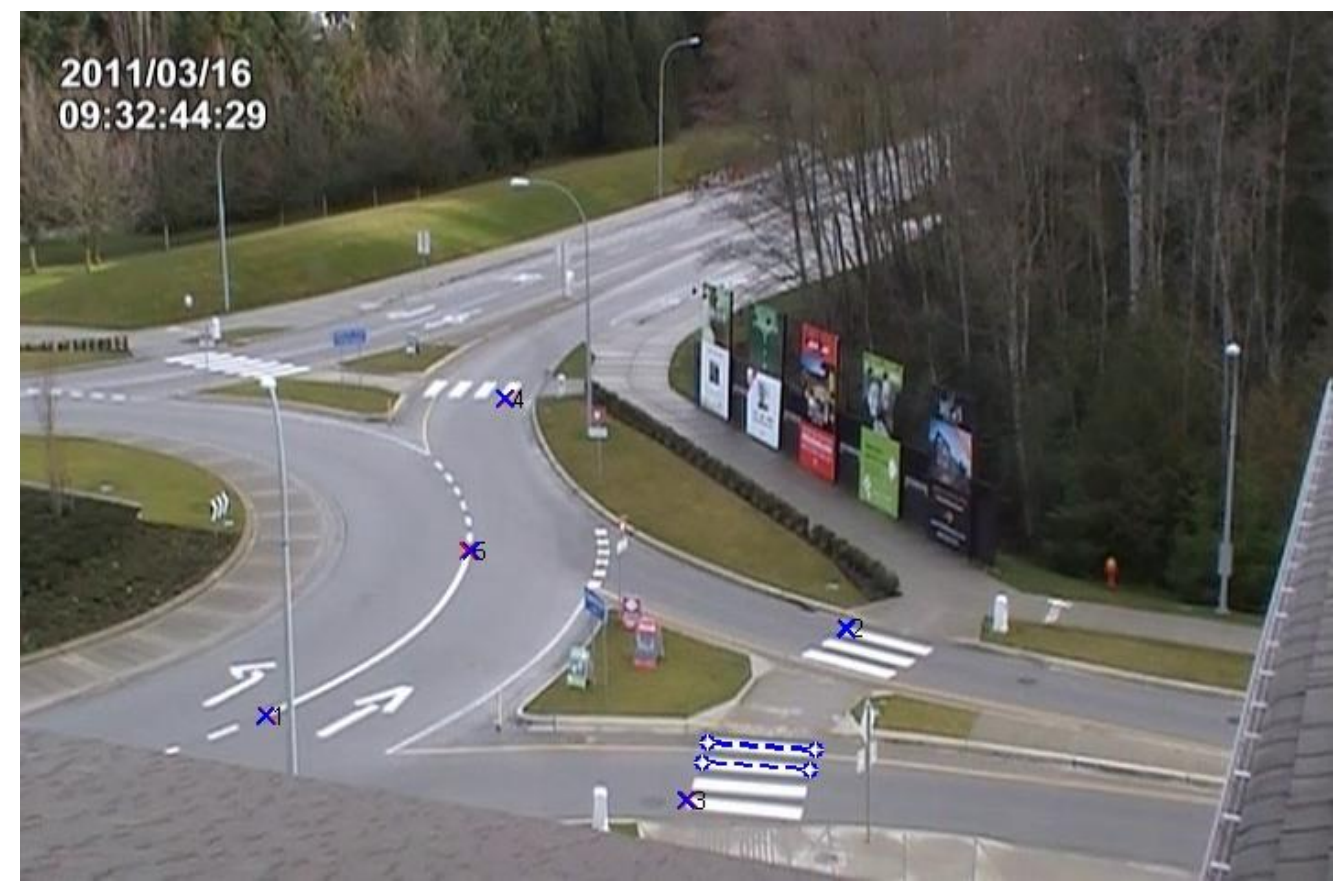

(a) 


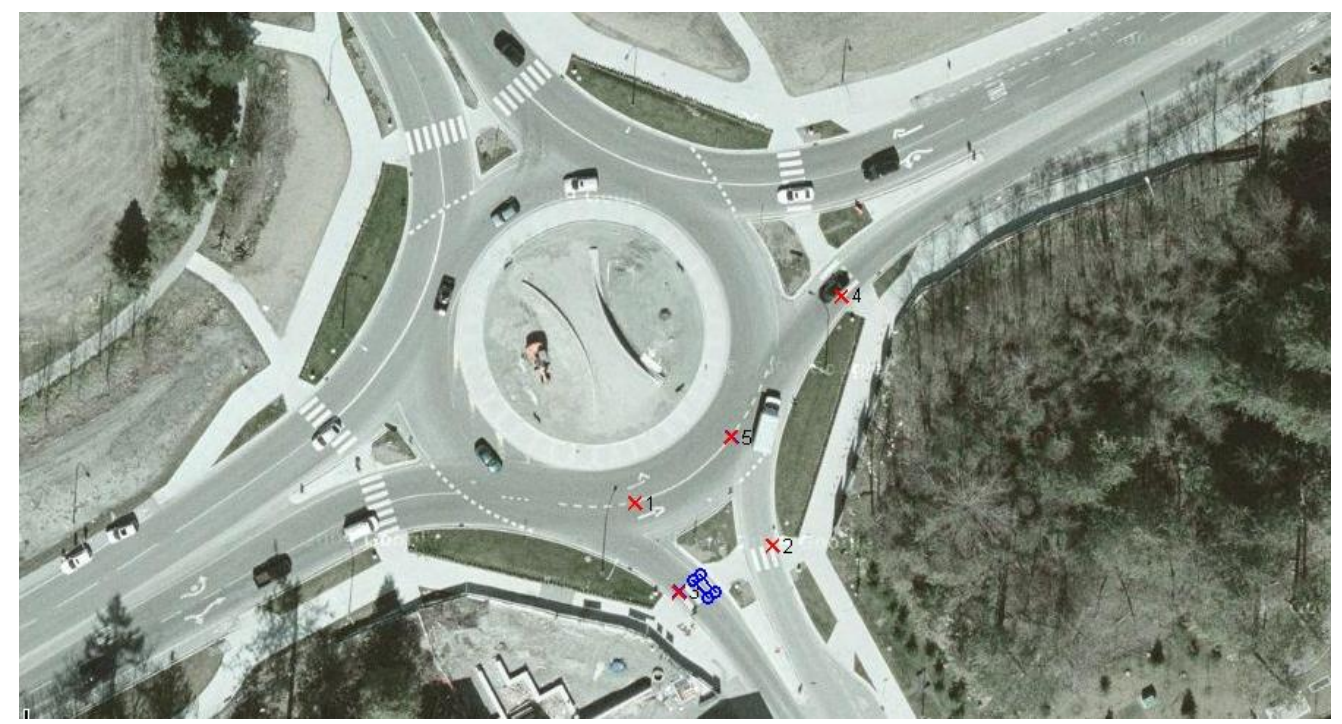

(b)

Figure 3.2 Feature points and distance lines used to link (a) the video image, with (b) the world image (maps data, Google, 2011).

Figures 3.3 shows square grids plotted in the video and world image which show a very good match.

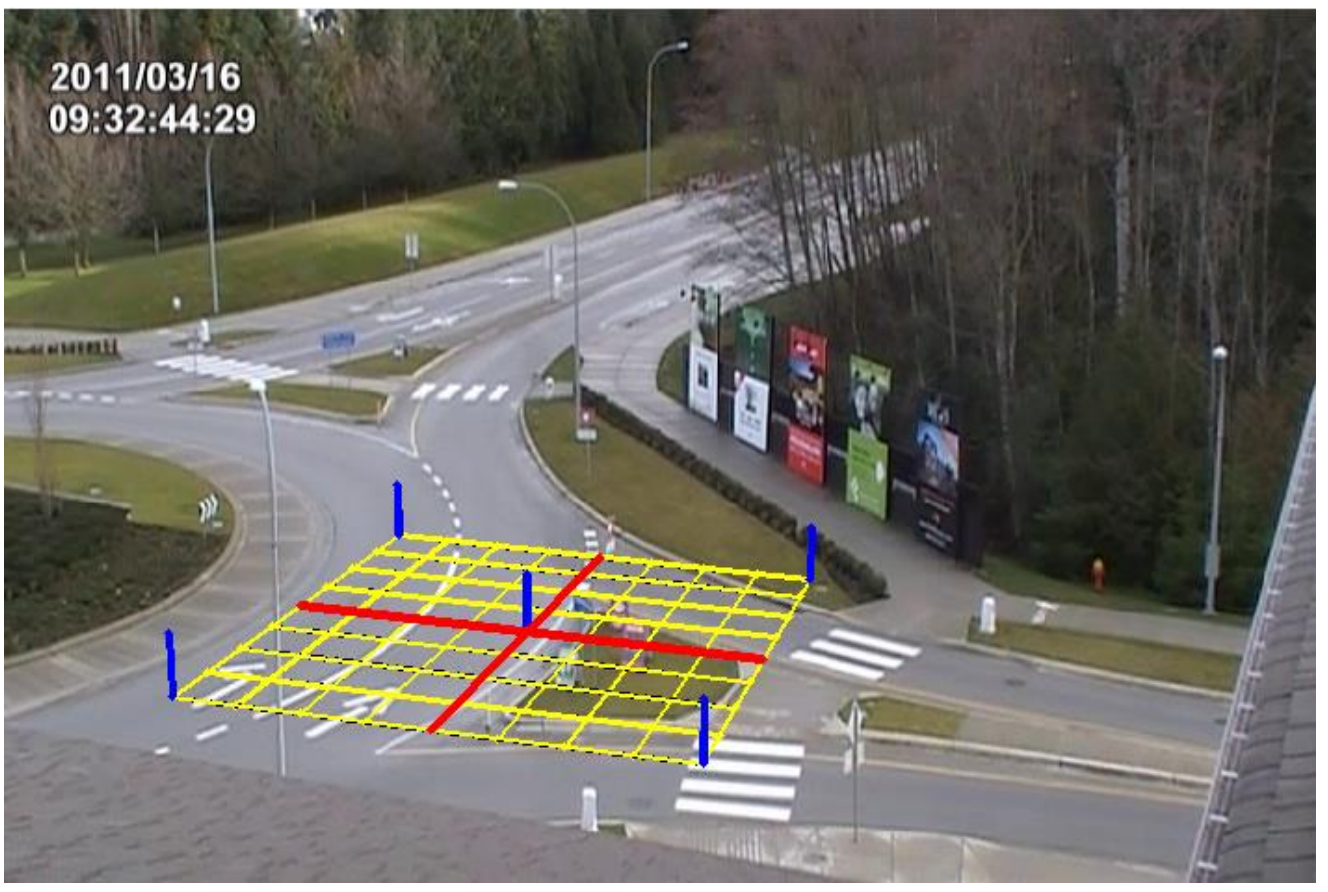


(a)

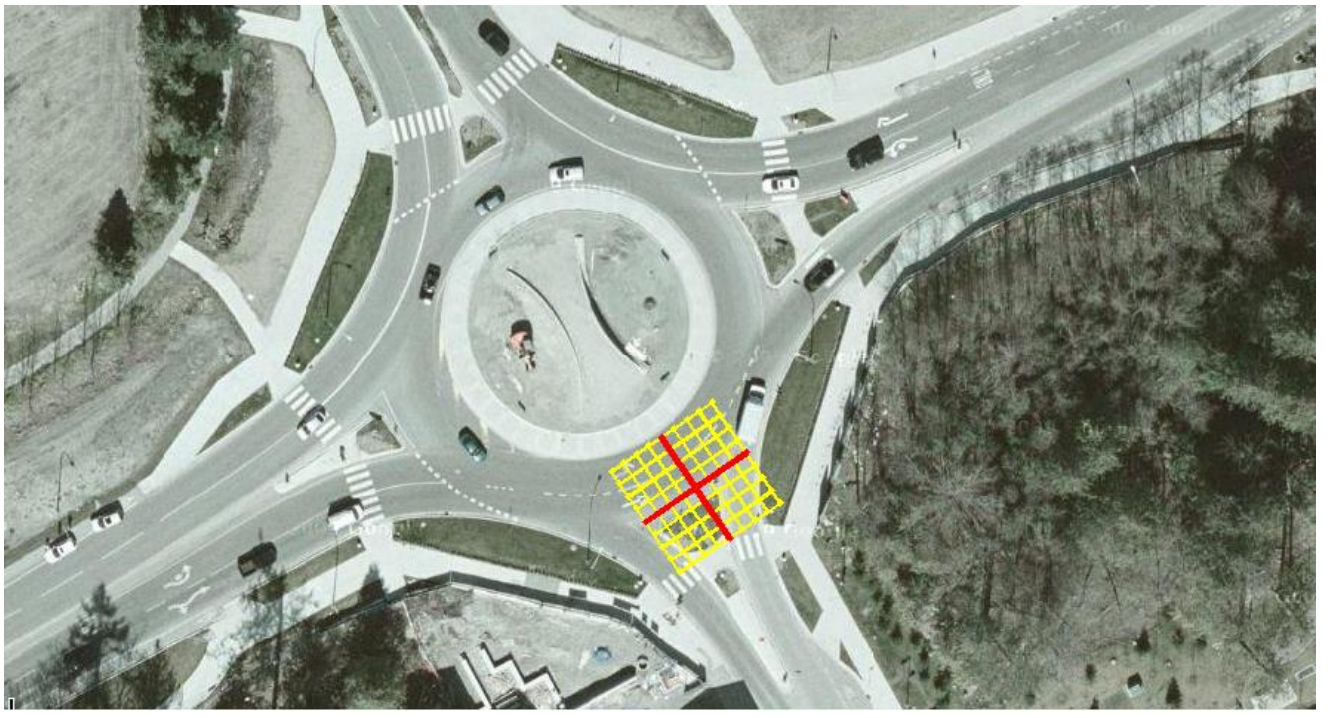

(b)

Figure 3.3 Use of a square grid to validate the calibration process between (a) the video image, and (b) the world image.

The world image was imported from the Google maps website (2011). The world image is 897 pixels by 484 pixels. The actual distances covered in the world image are 172.5 meters by 93.1 meters, and the pixel per meter value is calculated by diving 897 pixels by 172.5 meters which is equal to 5.2 pixels per meter.

\subsubsection{Background Generation}

The UBC automated traffic safety tool requires a background image to be used in the object detection and tracking process. The background image should be clear of all road users in order to be used in the feature tracking process. Background generation scripts have been used to generate a background per each 10 minutes in the video segment, and it is generated automatically using an averaging criterion of all image pixels in the frames. Figure 3.4 shows an example of the backgrounds generated for scene one on day one. The background generation process has also been applied for all scenes on both days. 

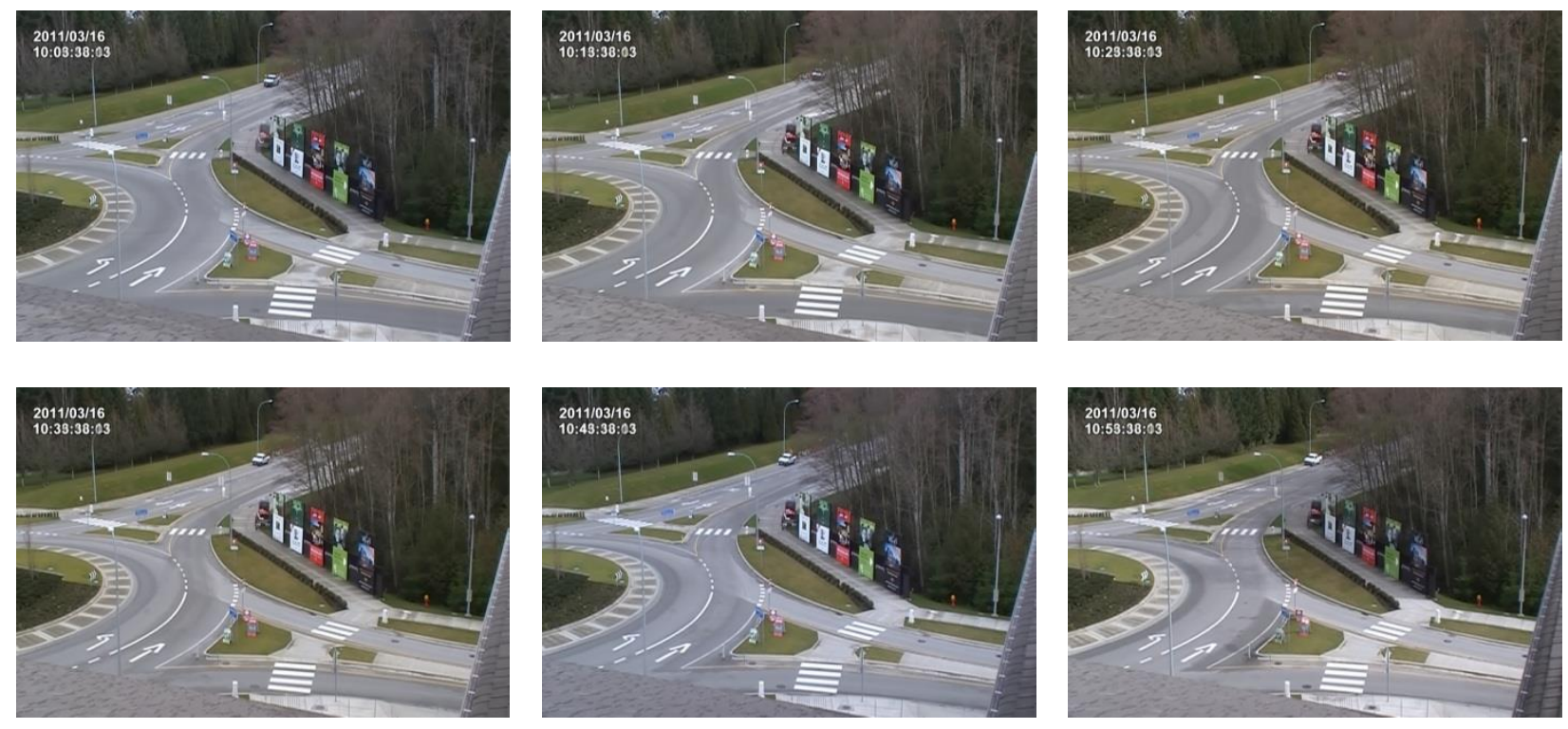

Figure 3.4 Example of backgrounds generated (scene one, day one) with six backgrounds for each one-hour video.

\subsection{Automated Safety Analysis Tool}

\subsubsection{Feature Tracking and Grouping}

The UBC automated safety tool is used for the feature tracking of the video components. Feature tracking is the automated process performed to identify the features in the video by comparing the video frames to the clear background generated from the previous step. Figure 3.5 shows an example of feature tracking of the second scene video component. 


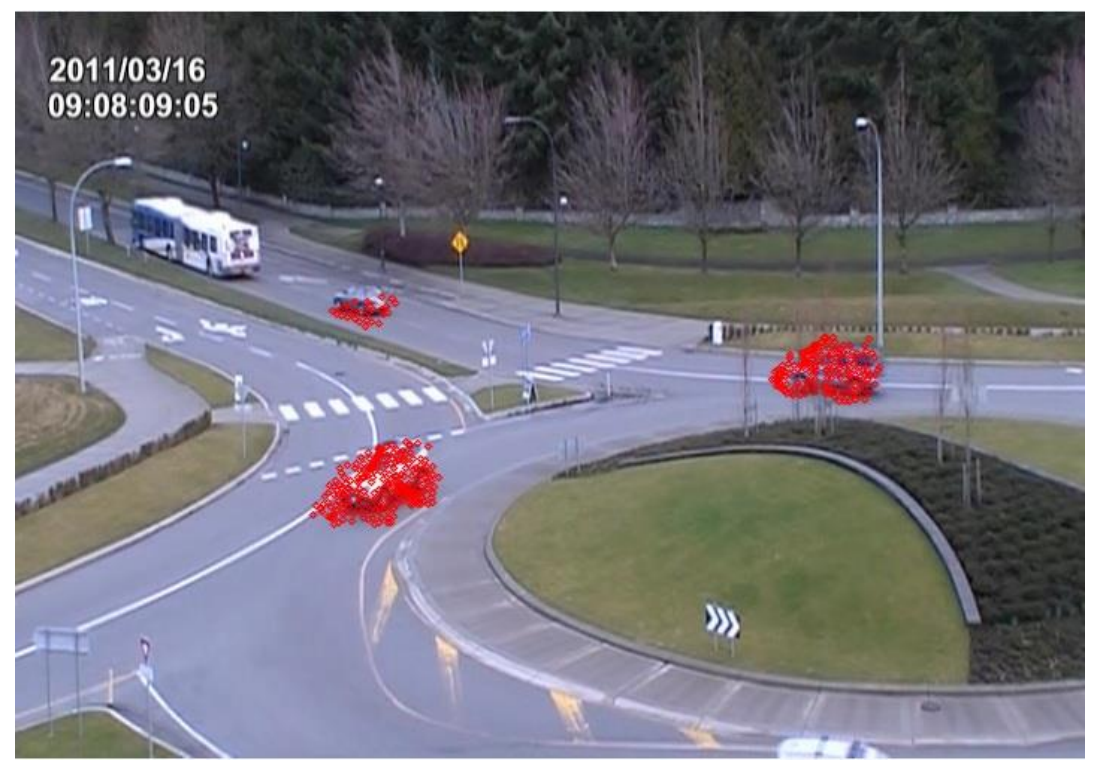

Figure 3.5 Example of feature tracking of the second scene video component

The feature grouping process, considered a key process that affects the quality of tracking for different road users, follows the feature tracking process. The number of features, the distances between them, the assumed vehicle size, the feature velocity, along with other parameters are used to improve the feature grouping. Figure 3.6 shows an example of the objects generated for the vehicle features. The details of feature tracking and grouping algorithms can be found in the research paper done by Saunier \& Sayed (2006) 


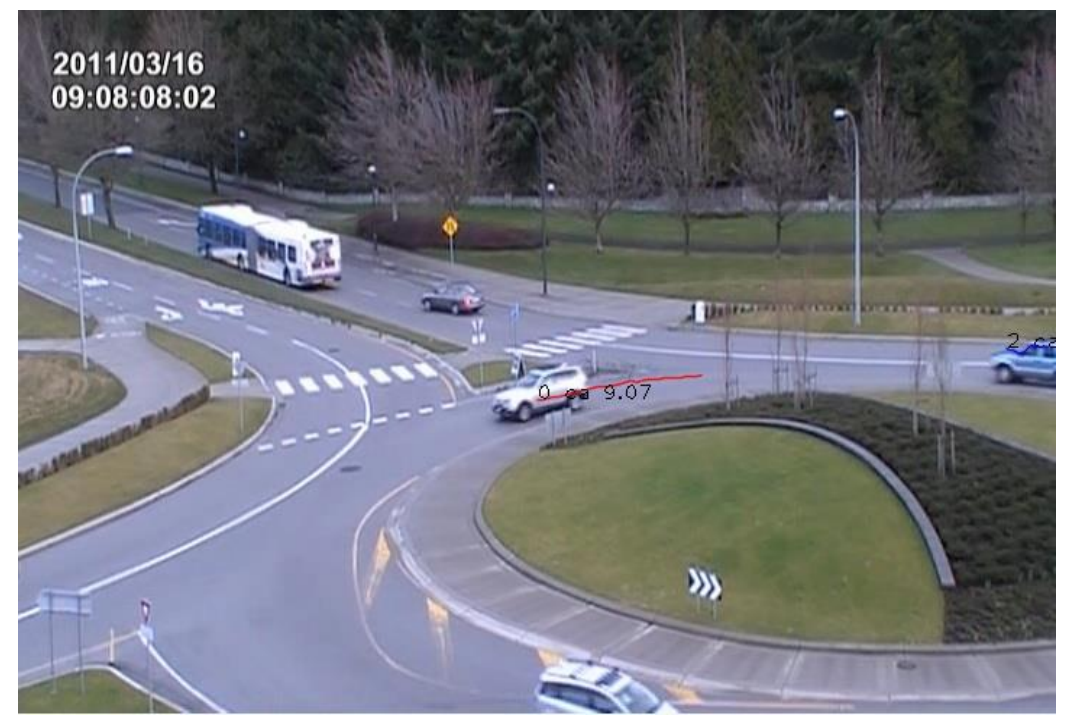

Figure 3.6 Example of feature grouping of a vehicle in a video component of scene two

A mask image is used for each scene to shade some parts of the video image and focus the analysis on a particular interaction. Features within the un-shaded areas are only considered in the feature tracking process. Figure 3.7 shows an example of the mask image used in scene two to focus on vehicle-vehicle interactions.

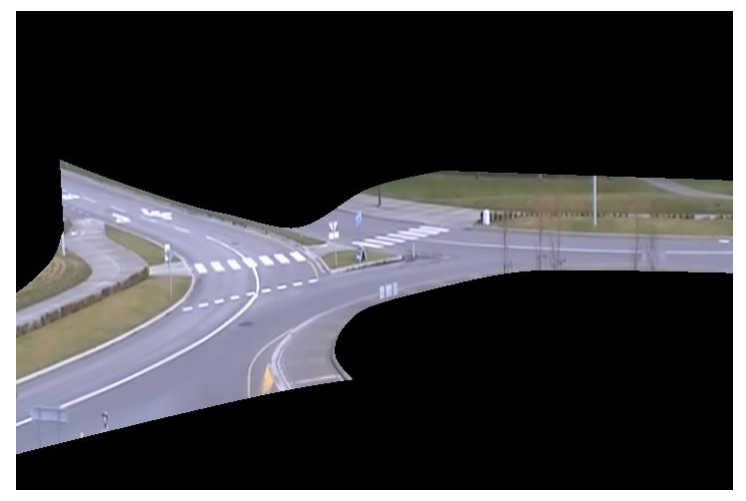

Figure 3.7 Example of mask image used in second scene to focus on vehicle-vehicle interactions 


\subsubsection{Prototype Generation}

Conflicts are generated in this research using the advanced probabilistic frame work proposed by Saunier, Sayed, \& Ismail (2010). The use of this methodology requires projections of vehicle positions based on previous general vehicle patterns. The process of generation of the previous patterns is called prototypes' generation. Ten minutes of each video scene on each day have been analyzed using the UBC automated traffic safety tool to generate prototypes. Figure 3.8 shows an example of prototypes generated for vehicle-pedestrian conflicts in scene one on day one in video and world images. 


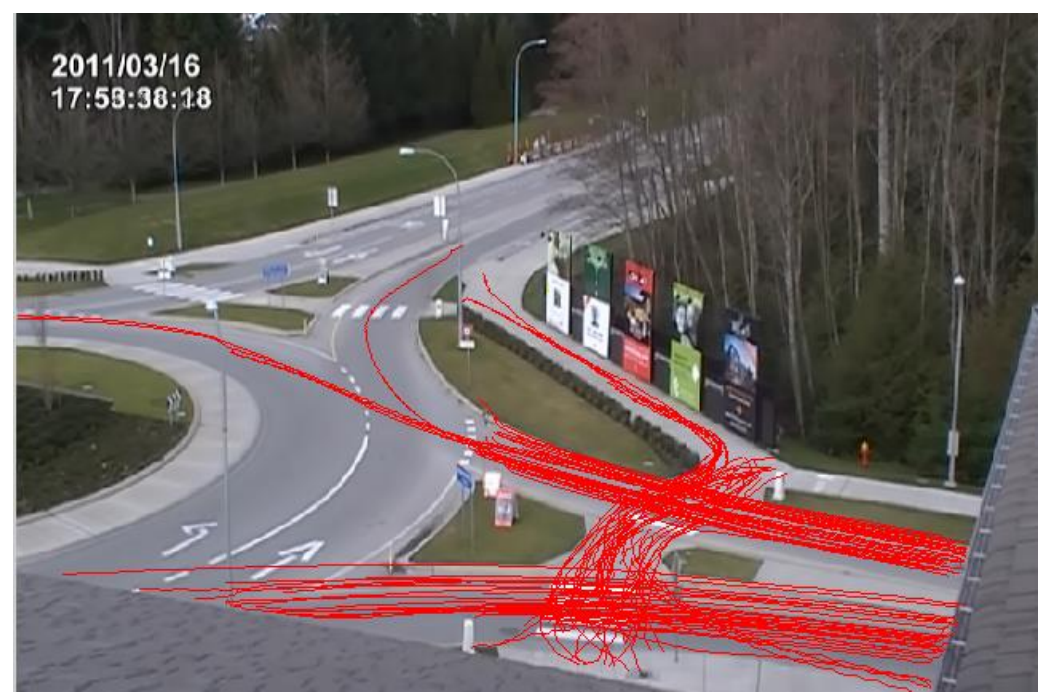

(a)

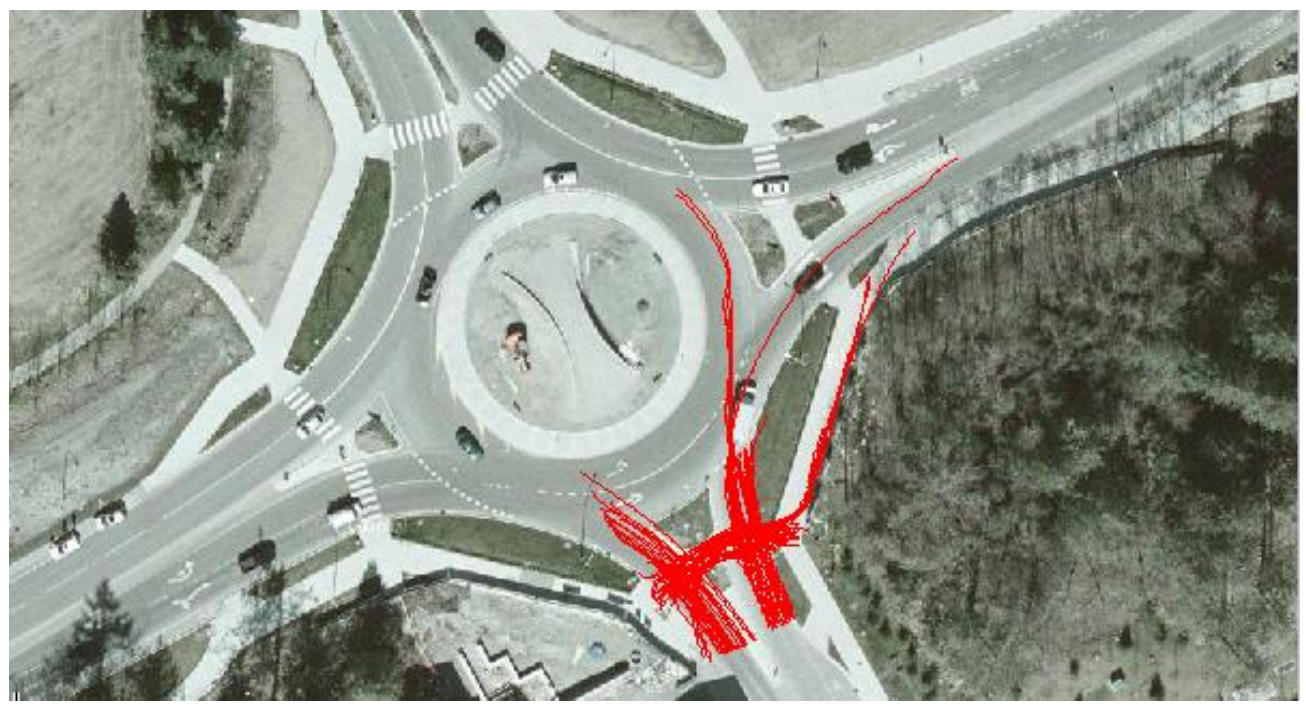

(b)

Figure 3.8 Example of prototypes generated for scene one (day one) for vehicle-pedestrian conflicts plotted on (a) the video image, and (b) the world image.

\subsubsection{Event Generation}

The probabilistic calculation of TTC indicator will be used in generating events as detailed in the previous work for Saunier, Sayed, \& Ismail (2010). The probabilistic approach is based on extrapolating position of the road user, based on their current position and speed. Generated 
prototypes shown in the previous section are used to estimate the potential conflict points between each of the two road users. The UBC automated safety tool uses the probabilistic approach in generating TTC events, and the objects generated by the tool are assigned to prototypes using the LCSS algorithm with some constraints (e.g. maximum distance and history of prototype assignment). An event between two road users is considered if their future positions coincide spatially (within one meter) and temporally. Based on this approach, TTC between two concurrent objects are calculated with a maximum time projection of four seconds. In order to focus on severe events only, events with a TTC less or equal to 1.5 seconds are only considered in the analysis. The proposed methodology has been applied for the five camera angles for both days, and the results and discussion are presented in the following section. 


\section{Results and Discussion}

This Chapter introduces the results and discussions of the analysis of the video data. It includes the in-office analysis, classifications of conflicts, road users' interactions and conflict, and accident contributing factors.

\subsection{In-office Analysis}

In-office analysis has been performed by analyzing the roundabout operation and understanding the general safety issues Figure 4.1 shows two major issues found by the preliminary safety analysis of the roundabout and they are:

- The roundabout island marking in front of approach D usually confuses drivers

- The circulating vehicles on lane 2 are usually confused while exiting the roundabout to approach B. They usually keep circulating and then suddenly need to turn left and exit.

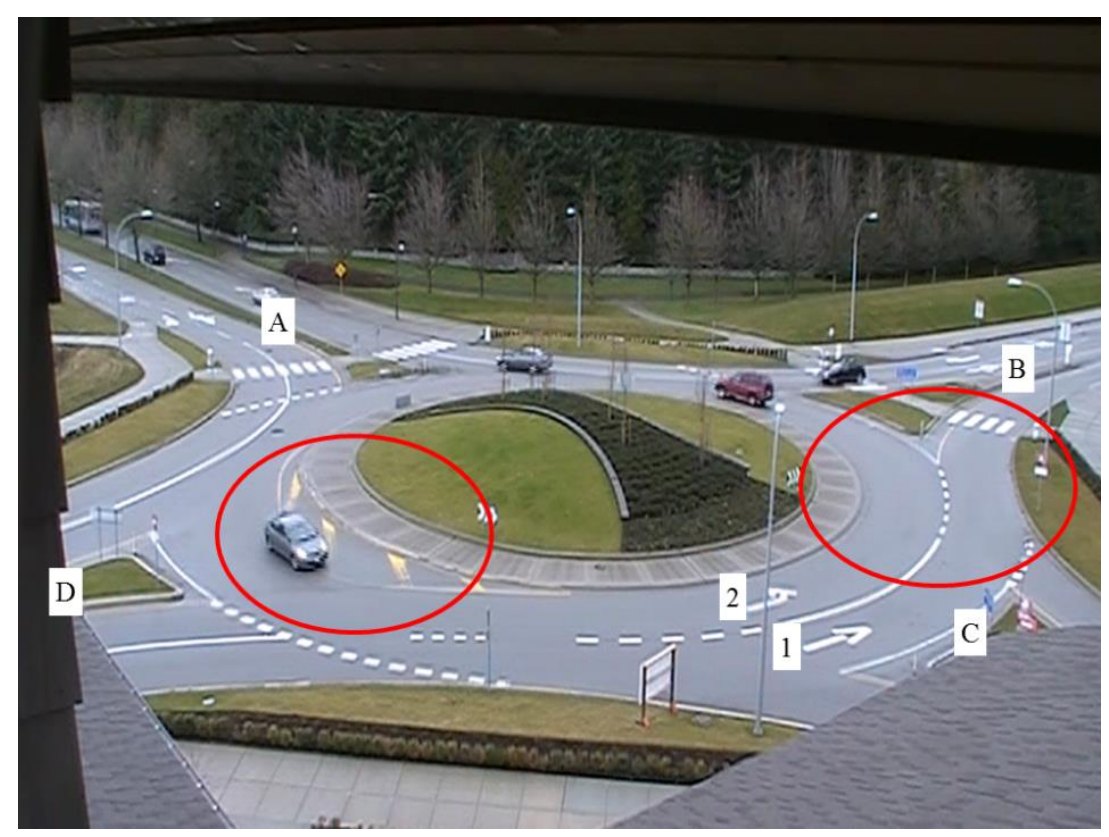

Figure 4.1 In-office analysis and the major issues found by the preliminary safety analysis of the roundabout. 
Vehicle traffic volume and pedestrian volume have been counted for two days. Figure 4.2 shows the traffic volume for Day 1 and Day 2. Detailed charts for traffic counts for vehicles and pedestrians per hour per approach are available in the appendices.

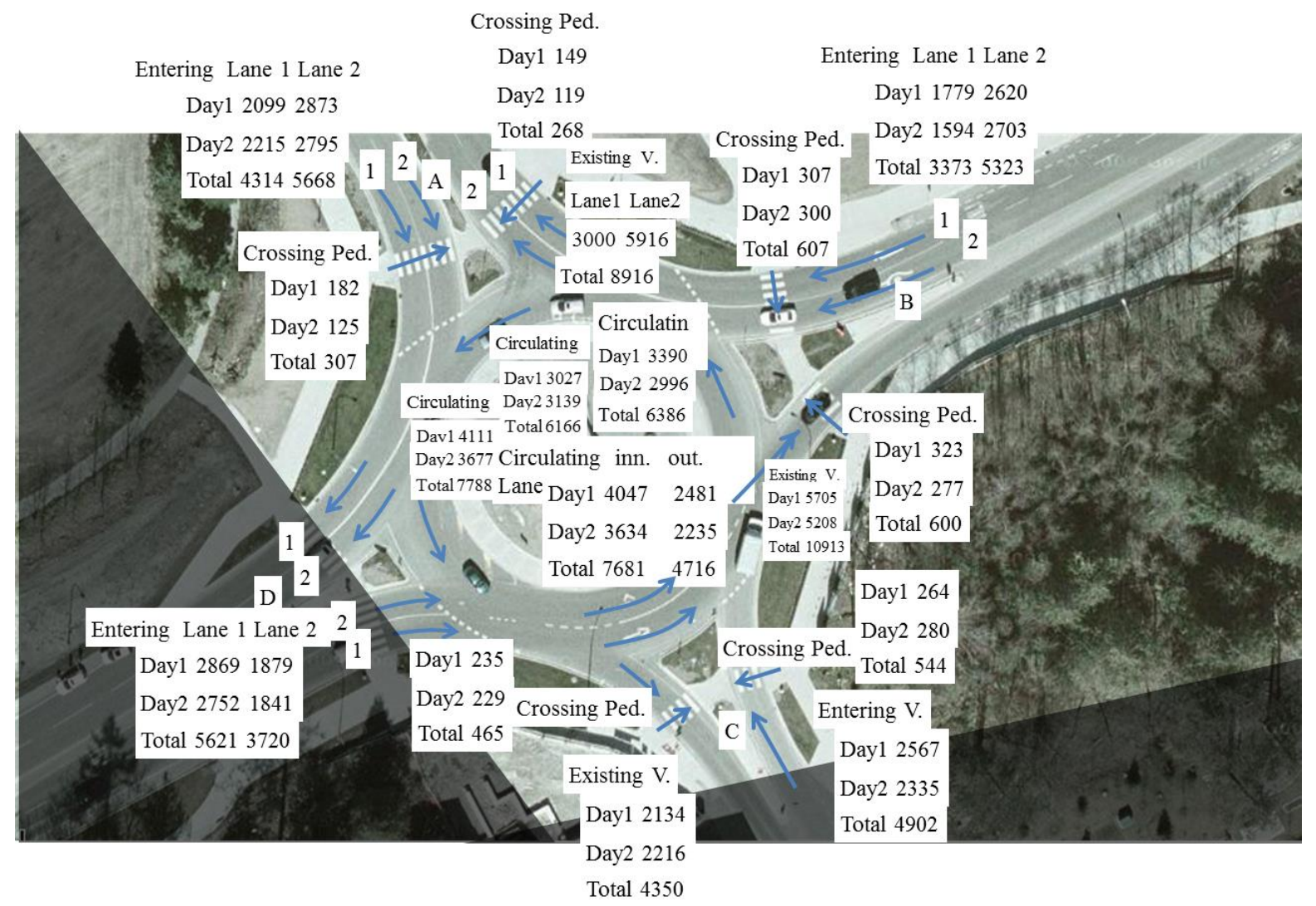

Figure 4.2 Volume count for vehicles and pedestrians (9:00 am to 8:00 pm - two days).

Figure 4.3 shows the approximate counts for vehicle and pedestrian volume in the roundabout: A blue arrow represents approximately 2000 vehicles in the two days; and a red arrow represents approximately 200 pedestrians crossing in the two days. In these traffic counts, cyclists behaving as vehicles are counted as vehicles and cyclists behaving as pedestrians are counted as pedestrians. 


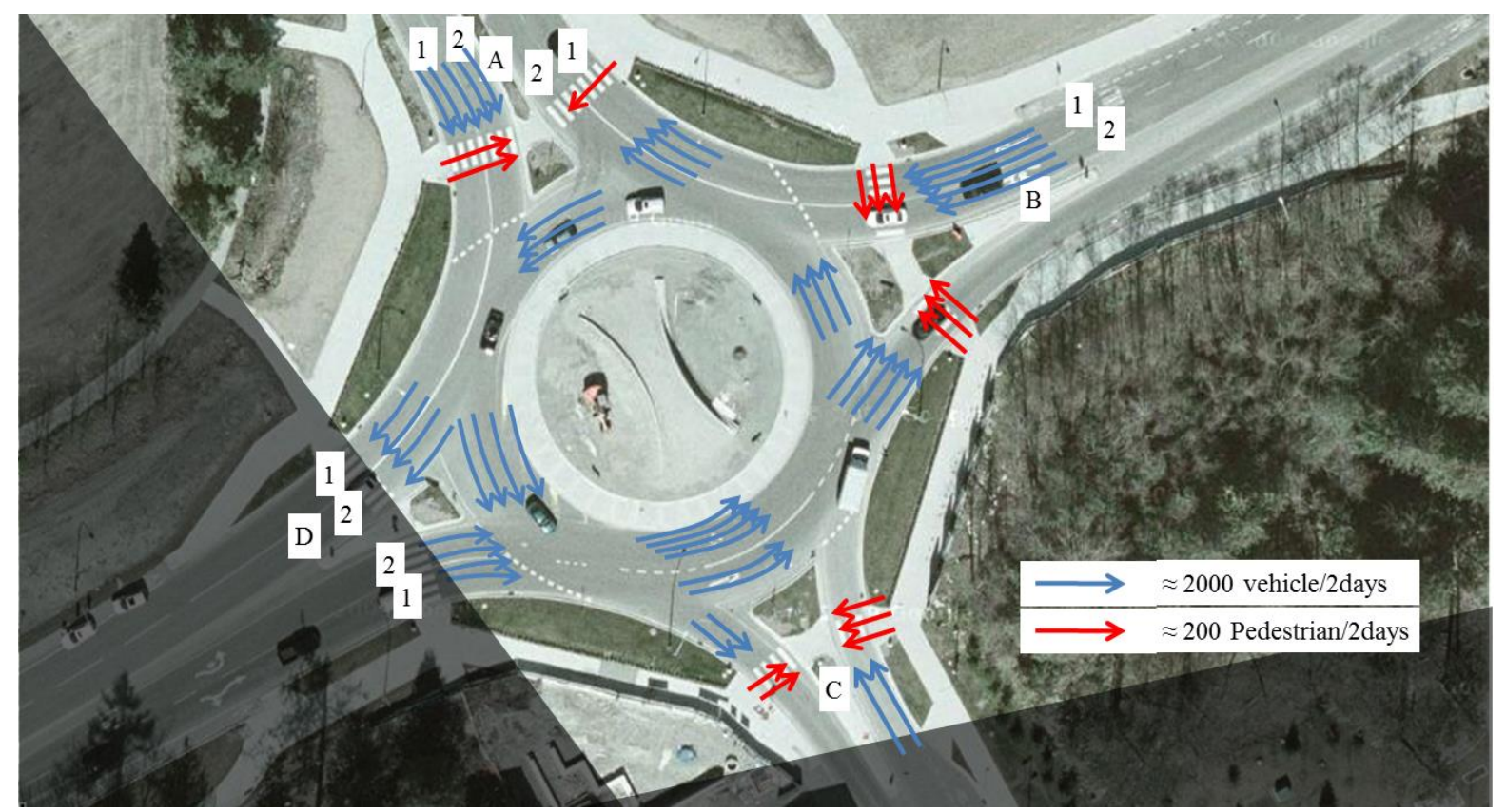

Figure 4.3 Volume count for vehicles and pedestrians as noted by arrows (two days).

\subsection{Conflict Classification}

As there are many conflicts generated by the UBC automated TCT, the conflicts have to be classified and reviewed. The classification of conflicts helps in identifying the over-represented clusters of traffic conflicts. Conflicts are classified in the following levels:

1. Vehicle-vehicle conflicts:

- Entering-circulating conflicts

- Exiting-circulating conflicts

- Rear-end conflicts (occurring in entering, exiting, and circulating lanes)

- Sideswipe conflicts

2. Vehicle-pedestrian conflicts:

- Entering-vehicle-pedestrian crossing conflicts

- Exiting-vehicle-pedestrian crossing conflicts 
3. Vehicle-cyclist conflicts

- Vehicle-cyclist conflicts where cyclists behave as vehicles

- Vehicle-cyclist conflicts where cyclists behave as pedestrians

4. Cyclist-pedestrian conflicts

5. Cyclist-cyclist conflicts

In each of the five conflicts level, the following analysis steps are performed:

1. The frequency of conflicts is calculated and compared among different approaches in the roundabout (where applicable).

2. For each conflict type and level, videos of conflicts will be manually reviewed to identify the possible causes of these conflicts.

3. The conflict frequency distribution will be plotted to identify patterns of conflicts severity indicators. Frequency distribution curves can be implemented to compare between approaches or between different conflicts types in the roundabout (where applicable).

The automated TCT produces a set of conflicts with different severity indicators presented in video segments. These conflicts can be used in several ways to diagnose the safety of the location.

The TTC indicator is used in this safety diagnosis to identify the conflicts. Any event between two road users with a TTC less than 1.5 seconds is considered a conflict. There were approximately 871 conflicts on the overall roundabout (except the crosswalk on approach D) over the period of two days. Figure 4.4 shows the five basic types of these conflicts and their frequency. Vehicle-vehicle conflicts were the most frequent, occurring 639 times (73.4\%), vehicle-pedestrian conflicts occurred 148 times (17\%), vehicle-cyclist conflicts occurred 69 
(7.9\%) times, and cyclist-cyclist conflicts and cyclist-pedestrian conflicts occurred five times $(0.6 \%)$, and ten (1.1\%) times, respectively.

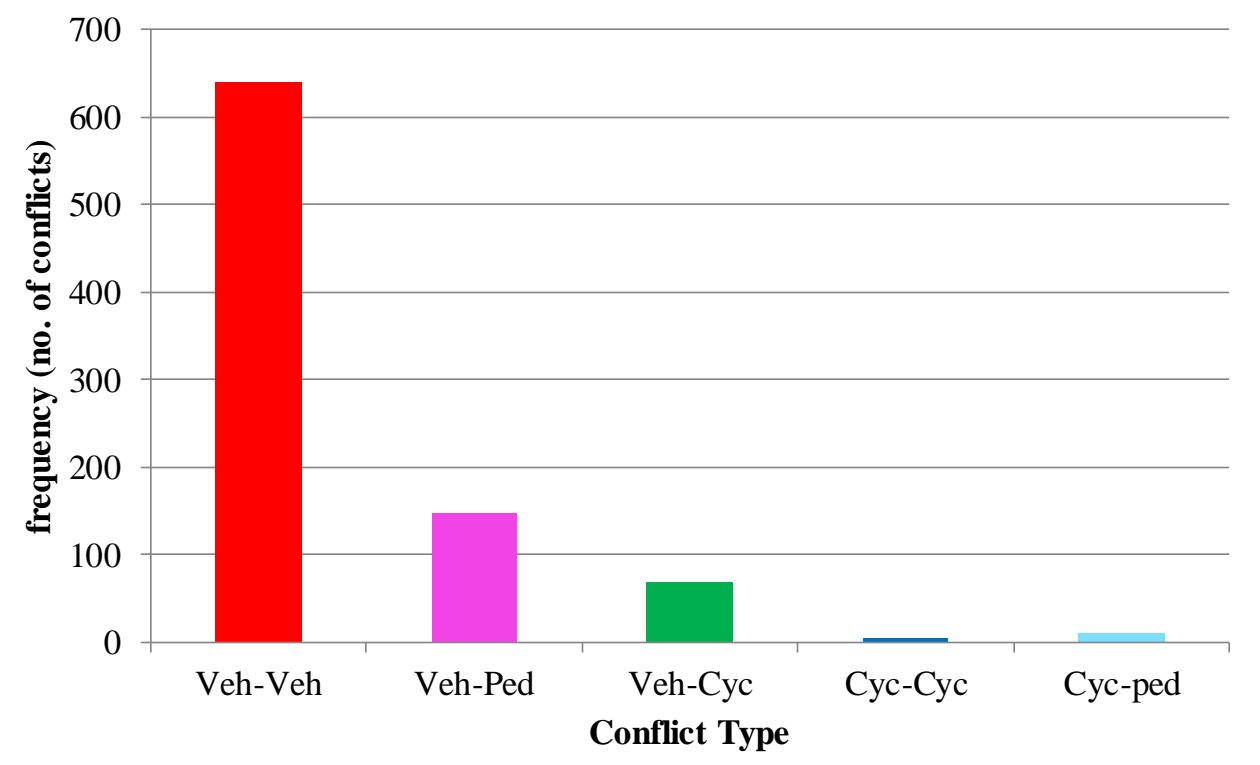

Figure 4.4 Frequency of different conflict types

As explained earlier in the event generation process, the conflict points are calculated based on the road user velocity, the assigned prototype, and the position. These conflict points are used as representatives for conflicts occurring at the roundabout, and they are used to pinpoint the safety issues occurring at different locations. Figure 4.5 shows the location of conflict points for all types. Colours of the conflict points are consistent with the colours in the previous bar chart. 


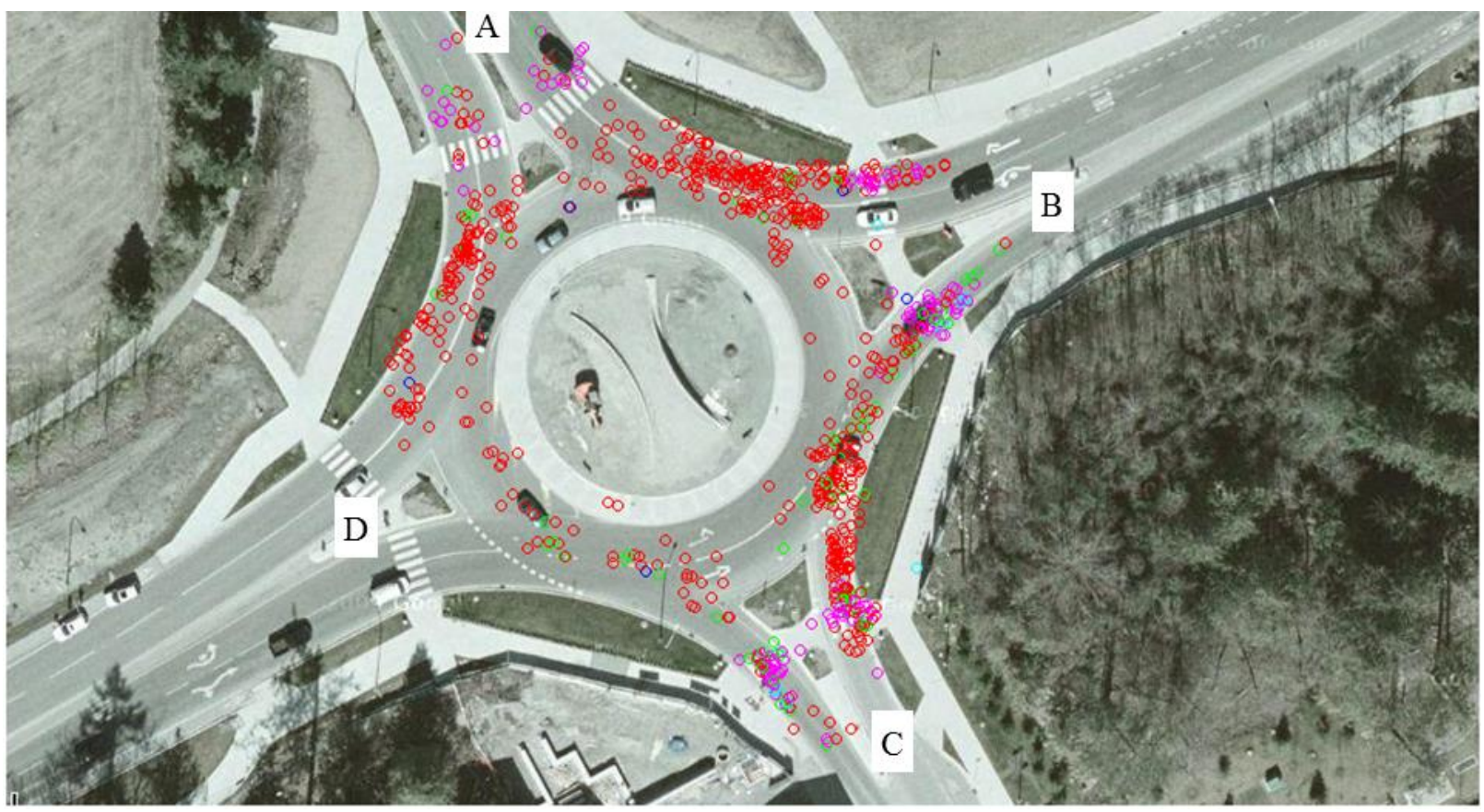

Figure 4.5 Location of conflict points for all types.

Figure 4.6 shows a heat map that shows conflict intensity (number of conflicts per square meter). It shows that conflicts are generally concentrated in entering and exiting lanes for approaches B and $\mathrm{C}$, with some areas reaching up to 10 conflicts per square meter. The heat map is helpful in generally locating areas with safety concerns in the roundabout. It also can be very helpful in aggregating the conflicts per time to be used in an online monitoring for road segments and intersections. 


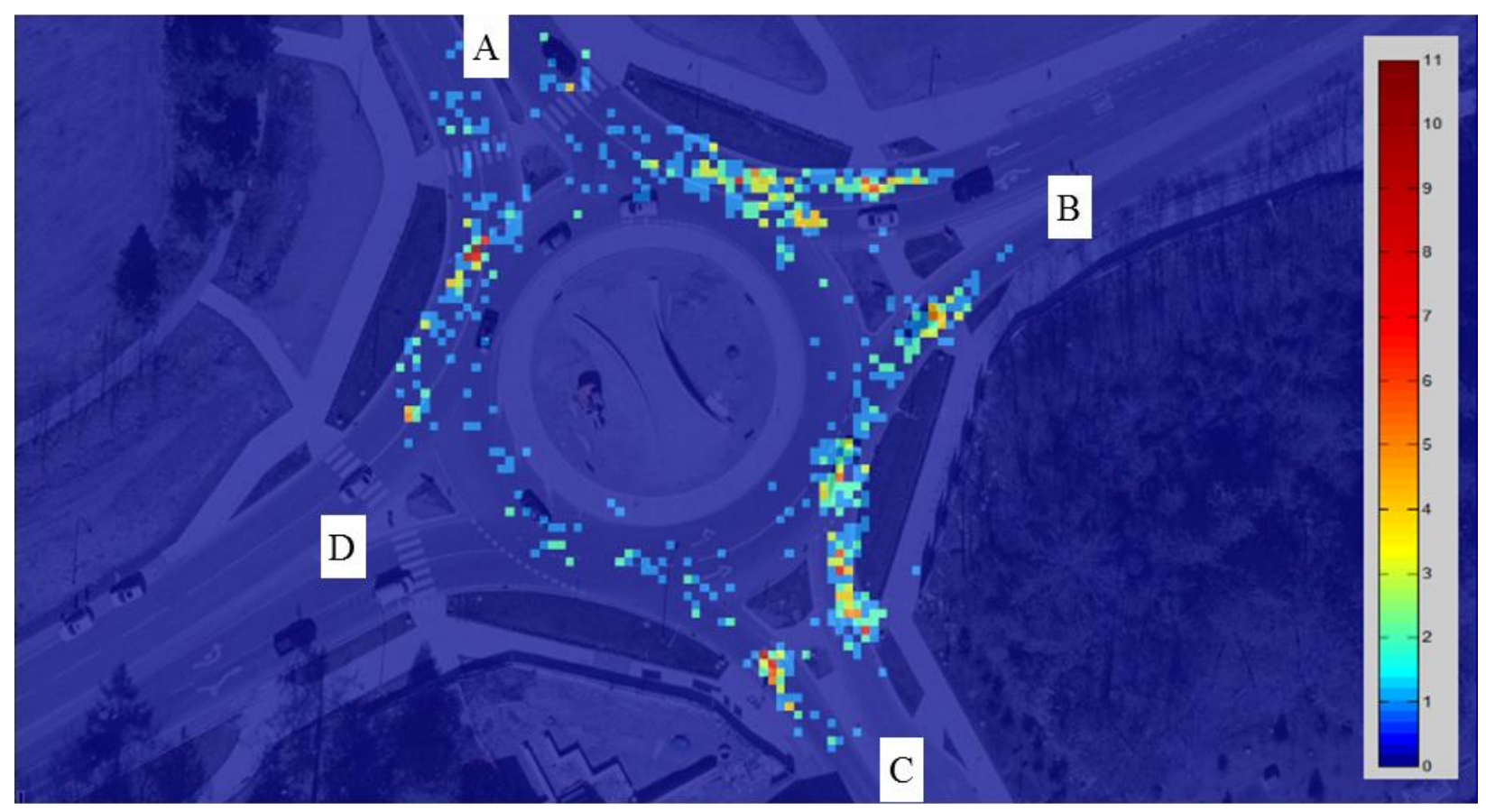

Figure 4.6 The heat map of all conflict points per area.

\subsection{Vehicle-vehicle Conflicts}

There are about 641 vehicle-vehicle conflicts in the roundabout, and these vehicle conflicts are further classified into several types as shown in Table 4.1. 
Table 4.1 Descriptions and figures of conflict types.

\begin{tabular}{l|l}
\multicolumn{1}{c}{ Conflict Type } & \multicolumn{1}{c}{ Description } \\
Entering-circulating & $\begin{array}{l}\text { An entering vehicle is } \\
\text { conflicting with a } \\
\text { circulating vehicle }\end{array}$ \\
Exiting-circulating & $\begin{array}{l}\text { An exiting vehicle is } \\
\text { conflicting with a } \\
\text { circulating vehicle or vice- } \\
\text { versa. } \\
\text { (entering) } \\
\text { (exiting) } \\
\text { (circulating) } \\
\text { A lead vehicle is } \\
\text { conflicting with a } \\
\text { following vehicle } \\
\text { (both vehicles are } \\
\text { conflicting in the entry } \\
\text { lanes, exit lanes, or } \\
\text { circulating lanes) } \\
\text { Sideswipe } \\
\text { A circulating vehicle is } \\
\text { conflicting with another } \\
\text { vehicle that moves in the } \\
\text { same direction }\end{array}$ \\
\hline
\end{tabular}

Figure 4.7 shows the total frequency of each vehicle-vehicle conflict type; entering-circulating conflicts are the most frequent which occurred 288 times (45.1\%). Exiting-circulating conflicts occurred 62 times (9.7\%); rear-end conflicts occurred 159 times (24.9\%), 63 times (9.9\%), and 42 times $(6.6 \%)$ in entering lanes, exiting lanes, and circulating lanes, respectively. The remainder of vehicle-vehicle conflicts involved the sideswipe type and that occurred 25 times $(3.9 \%)$ in the circulating lanes. 


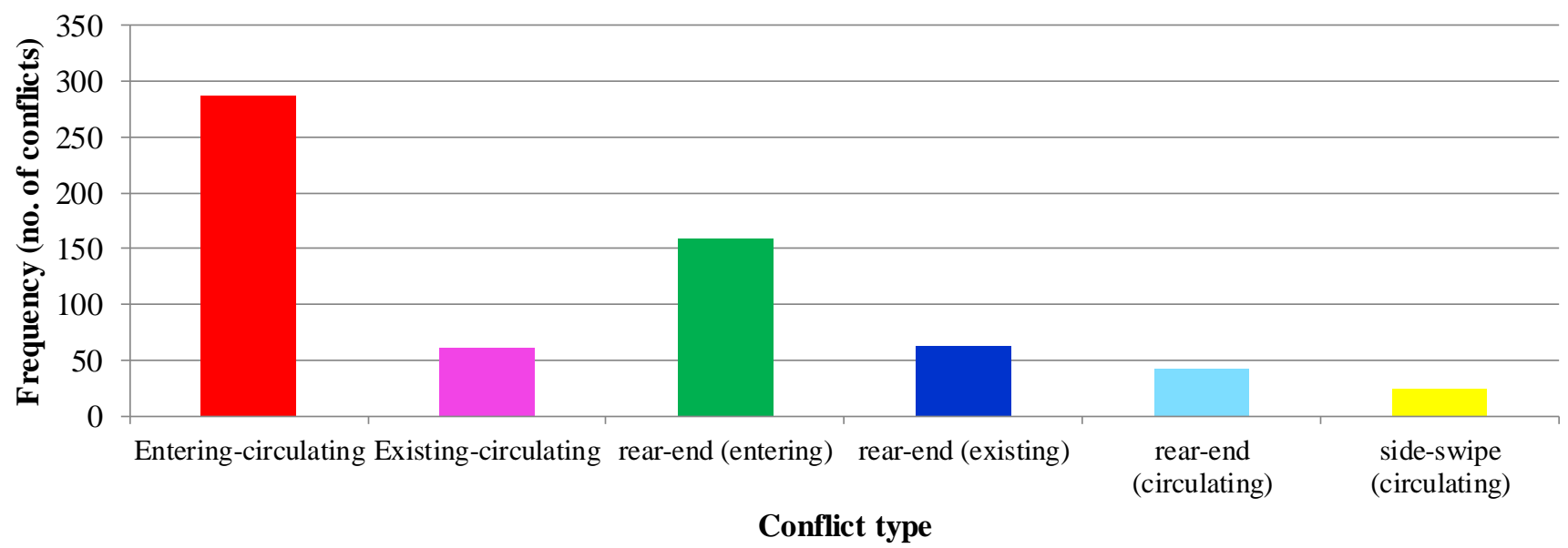

Figure 4.7 Frequency of each vehicle-vehicle conflict types

Figure 4.8 shows the conflicts points for each subtype in the vehicle-vehicle conflicts. Each type of vehicle-vehicle conflicts will be further analyzed in the following sections. Colours of conflict points are consistent with the colours in the previous bar chart.

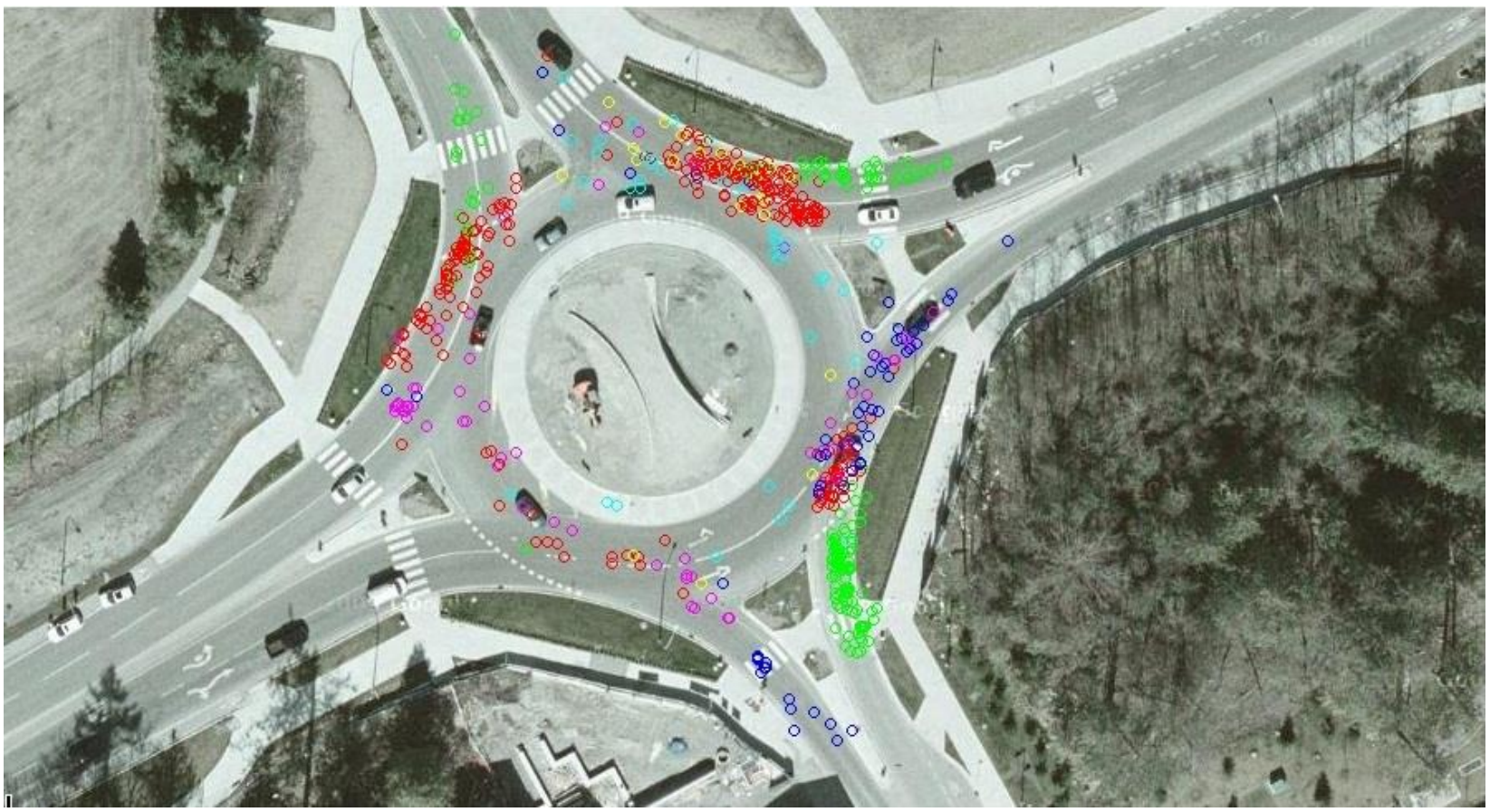

Figure 4.8 Conflict points of all vehicle-vehicle conflicts. 


\subsubsection{Entering-circulating Conflicts}

There are approximately 288 entering-circulating conflicts in the roundabout, and the conflict points are shown in Figure 4.9.

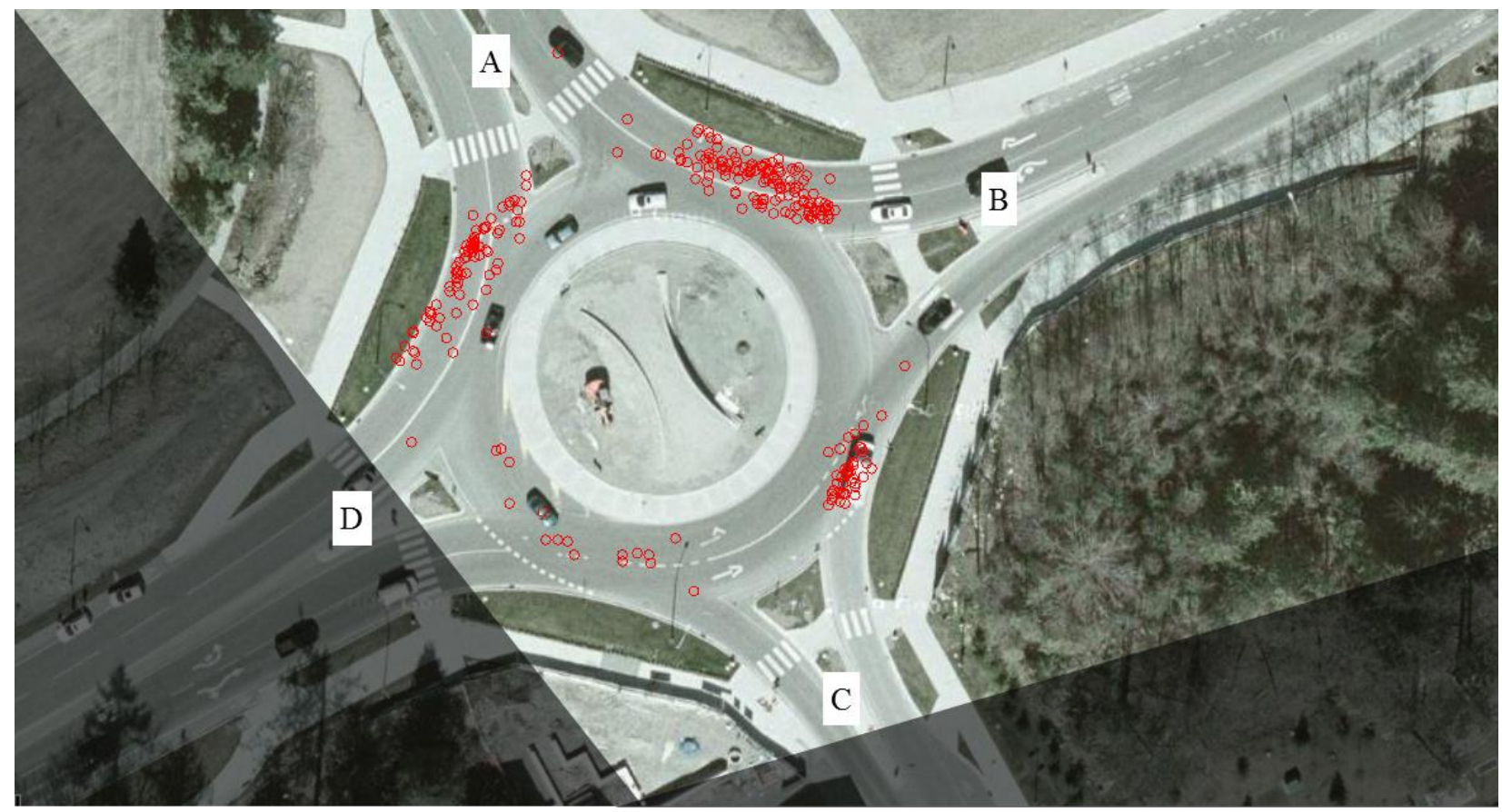

Figure 4.9 Entering-circulating conflict points.

To better diagnose the safety in the roundabout, it is important to compare the enteringcirculating conflicts across all approaches of the roundabout. This requires that the number of conflicts in all approaches be normalized for exposure. The exposure measure is computed by considering the maximum theoretical number of events. As an example, the exposure of entering-circulating traffic conflicts at approach $\mathrm{C}$ is calculated as follows: 
Exposure $($ in 1000 vehicles $)=\frac{\sqrt{\text { Entering vehicle volume } \times \text { Circulating vehicle volume }}}{1000} \quad$ Eq. 3

The counts of entering and circulating volume along with the conflict diagram are shown in Figure 4.10. There are approximately 4902 entering vehicles in the two days, and there are approximately 7861 and 4716 circulating vehicles in the inner and outer lanes, respectively.

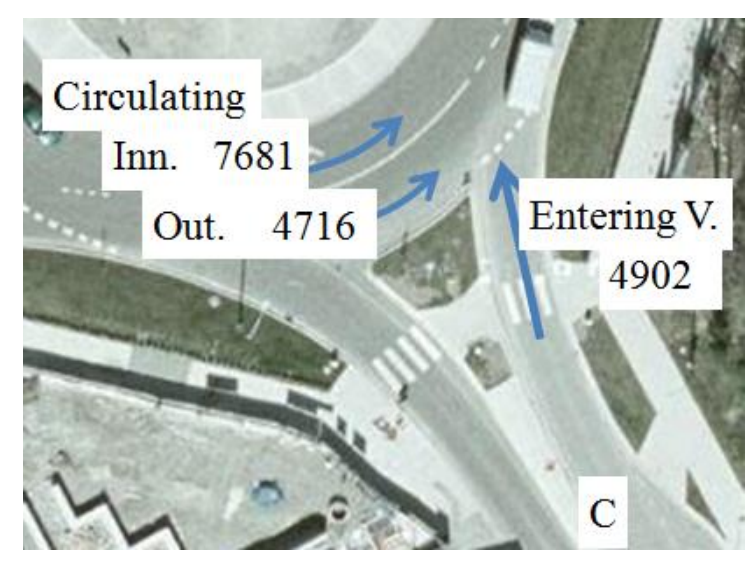

Figure 4.10 Volumes of entering and circulating vehicles (two days) on approach $\mathrm{C}$.

$$
\begin{aligned}
\text { Exposure }(\text { in } 1000 \text { vehicle }) & =\frac{\sqrt{4902 \times(7681+4716)}}{1000} \\
& =7.796 \text { maximum theoretical events in } 1000 \text { vehicles }
\end{aligned}
$$

The total number of entering-circulating conflicts generated by the automated tool for approach $\mathrm{C}$ is equal to 43 conflicts, and therefore, the conflicts per exposure rate for this particular conflict type on approach $\mathrm{C}$ will be:

Conflict rate $=$ no. of conflicts $/$ exposure $($ in 1000 vehicle $)=43 / 7.796$

$$
=5.52 \text { conflicts for one thousand vehicles }
$$

These calculations are repeated for entering-circulating conflicts for all approaches. The volumes of entering and circulating vehicles for all approaches are shown in Figure 4.11. 


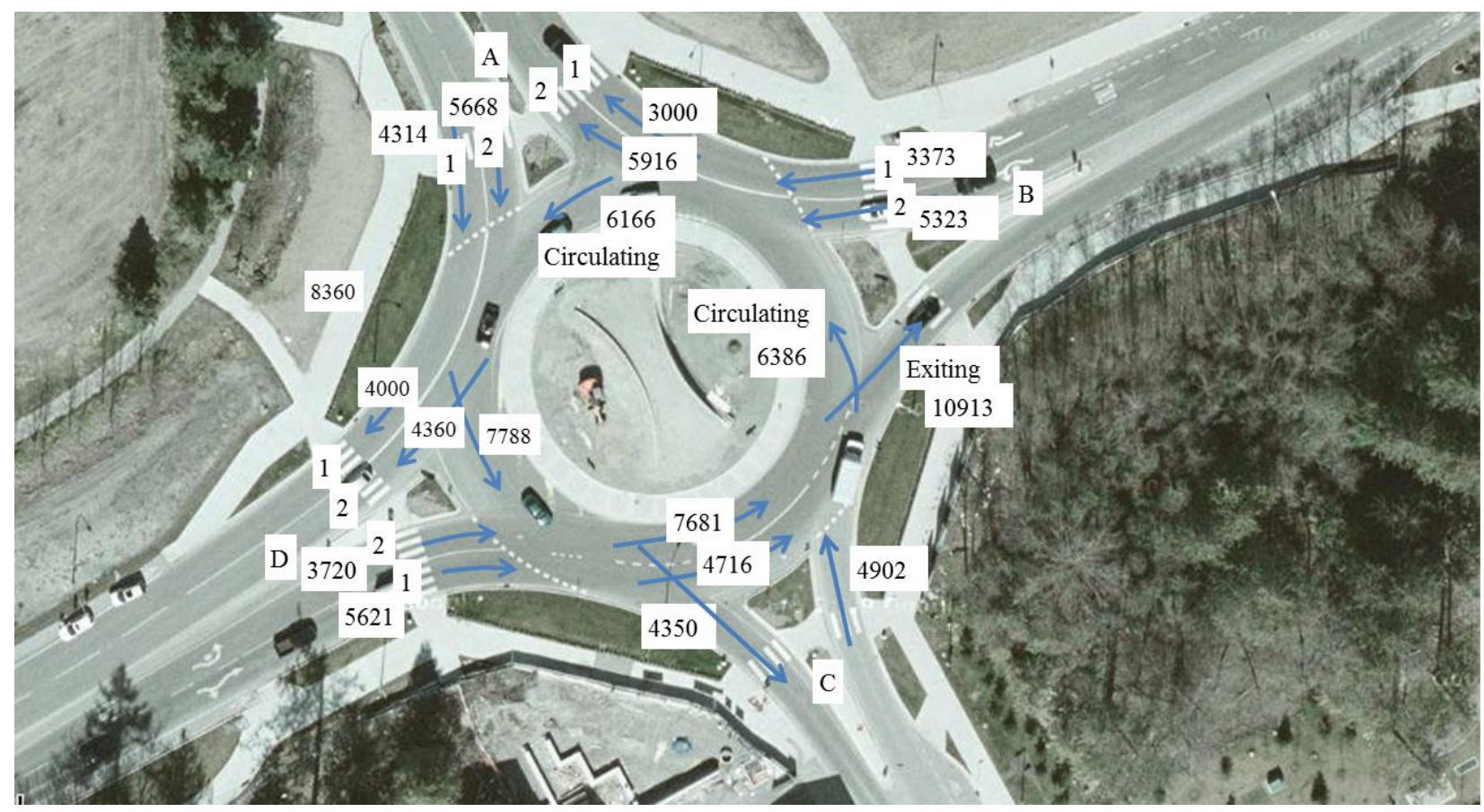

Figure 4.11 Volumes of entering and circulating vehicles (two days) for approaches A, B, C and D

Table 4.2 and Figure 4.12 show a comparison of the conflicts per exposure results for all approaches. The entering-circulating conflict rates for approaches A, B, C, and D are equal to $11.98,17.98,5.52$, and 1.99 conflicts per 1000 vehicles, respectively.

Table 4.2 Comparison of the entering-circulating conflicts in approaches A, B, C, and D.

Approaches

A $\quad$ B $\quad$ C $\quad$ D

Circulating 1

Circulating 2

Entering 1

Entering 2

Exposure (in 1000 vehicles)

No. of Entering-Circulating Conflicts

Conflict Rate

\begin{tabular}{|cccc|}
\hline 6166 & 6386 & 7681 & 7788 \\
0 & 0 & 4716 & 0 \\
4314 & 3373 & 4902 & 3720 \\
5668 & 5323 & 0 & 5621 \\
7.845 & 7.452 & 7.796 & 8.529 \\
94 & 134 & 43 & 17 \\
$\mathbf{1 1 . 9 8}$ & $\mathbf{1 7 . 9 8}$ & $\mathbf{5 . 5 2}$ & $\mathbf{1 . 9 9}$
\end{tabular}




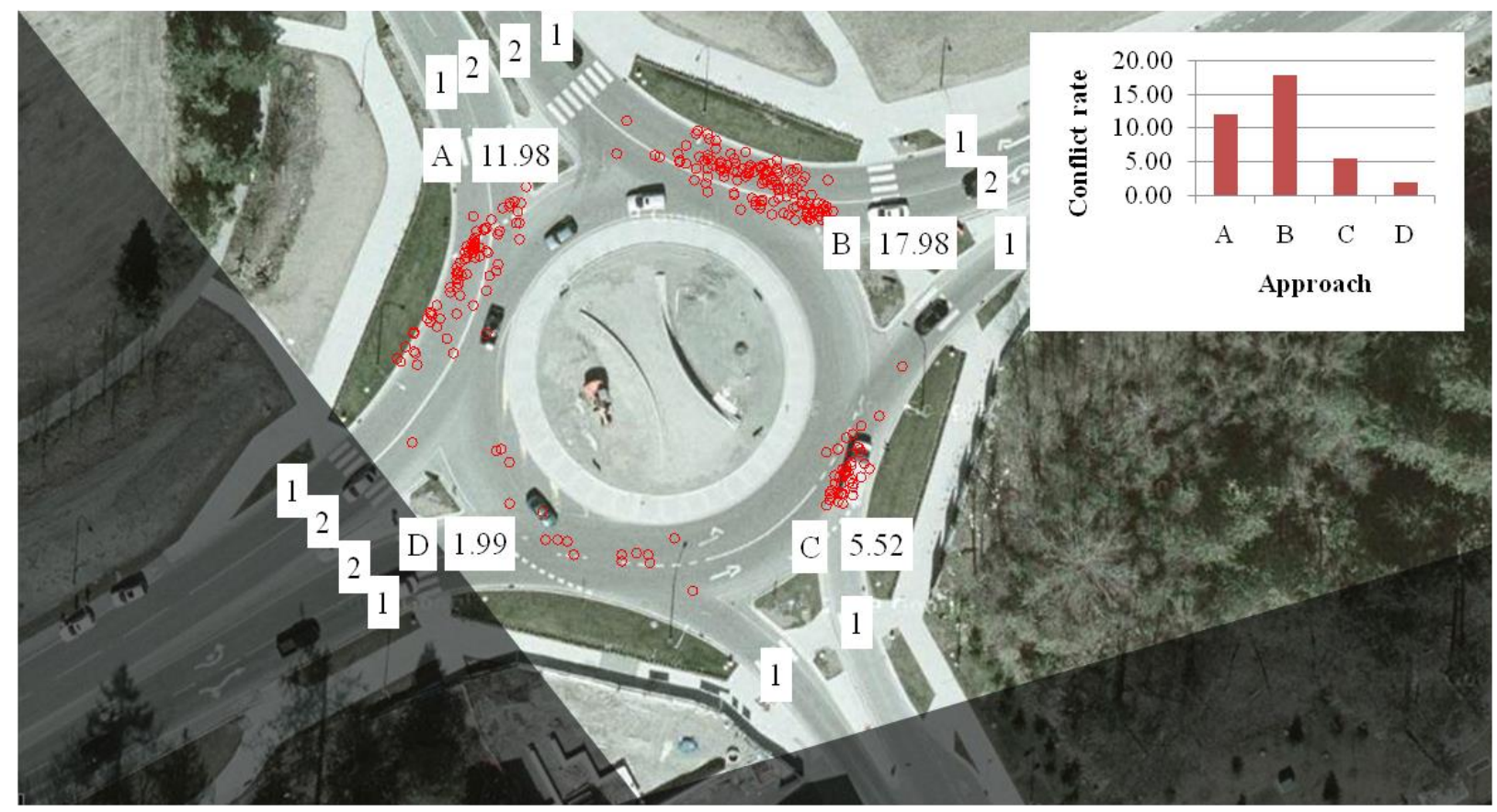

Figure 4.12 Comparison of the entering-circulating conflicts in approaches A, B, C, and D.

The conflict rates for entering-circulating conflicts for the geometric design of each approach can be used as a benchmark for comparison with other roundabouts.

Although approaches A and B have protected right turn lanes for entering vehicles, their conflict rates are more than double the conflict rates for vehicles entering from approaches $\mathrm{C}$ or $\mathrm{D}$. Most of entering-circulating conflicts for vehicles entering from approaches $\mathrm{A}$ and $\mathrm{B}$, as shown in Figure 4.9, are located on the protected right lane for entering vehicles. Even though the protected right-turn lanes for vehicles entering on approaches A and B from lane 1 may improve mobility of entering vehicles, it tends to generate more conflicts with circulating vehicles.

The conflict rates for entering-circulating conflicts on approaches $\mathrm{C}$ and $\mathrm{D}$ are less than approaches A and B. Right-turning entering vehicles have to yield for circulating vehicles because there is no protected right lane. Although this has helped reduce entering-circulating 
conflicts, it has resulted in generating exiting-circulating conflicts, as will be discussed in the second section.

By manually reviewing 94, 134, 141 and 37 entering-circulating conflicts on approaches A, B, C and D, respectively, the behaviour of motorists and conflict causes per approach are interpreted as follows:

- As shown by the conflict points in Figure 4.9, most of the conflict points for approaches $\mathrm{A}$ and $\mathrm{B}$ are located in entering lane 1. Vehicles entering from lane 1, as shown in Figure 4.13, usually hesitate when turning right if there is a circulating vehicle. This usually occurs because circulating vehicles are approaching the conflict area with the possibility of wrong exit negotiation. As there is only one circulating lane, entering vehicles anticipate that circulating vehicles will not negotiate properly when driving through exit lane 1 . This confusion may also occur because of the sign posted before the roundabout for entering vehicles. As shown in Figure 4.14, the sign informs drivers to yield for traffic in the roundabout. This sign is also posted at all entering lanes for all approaches. 


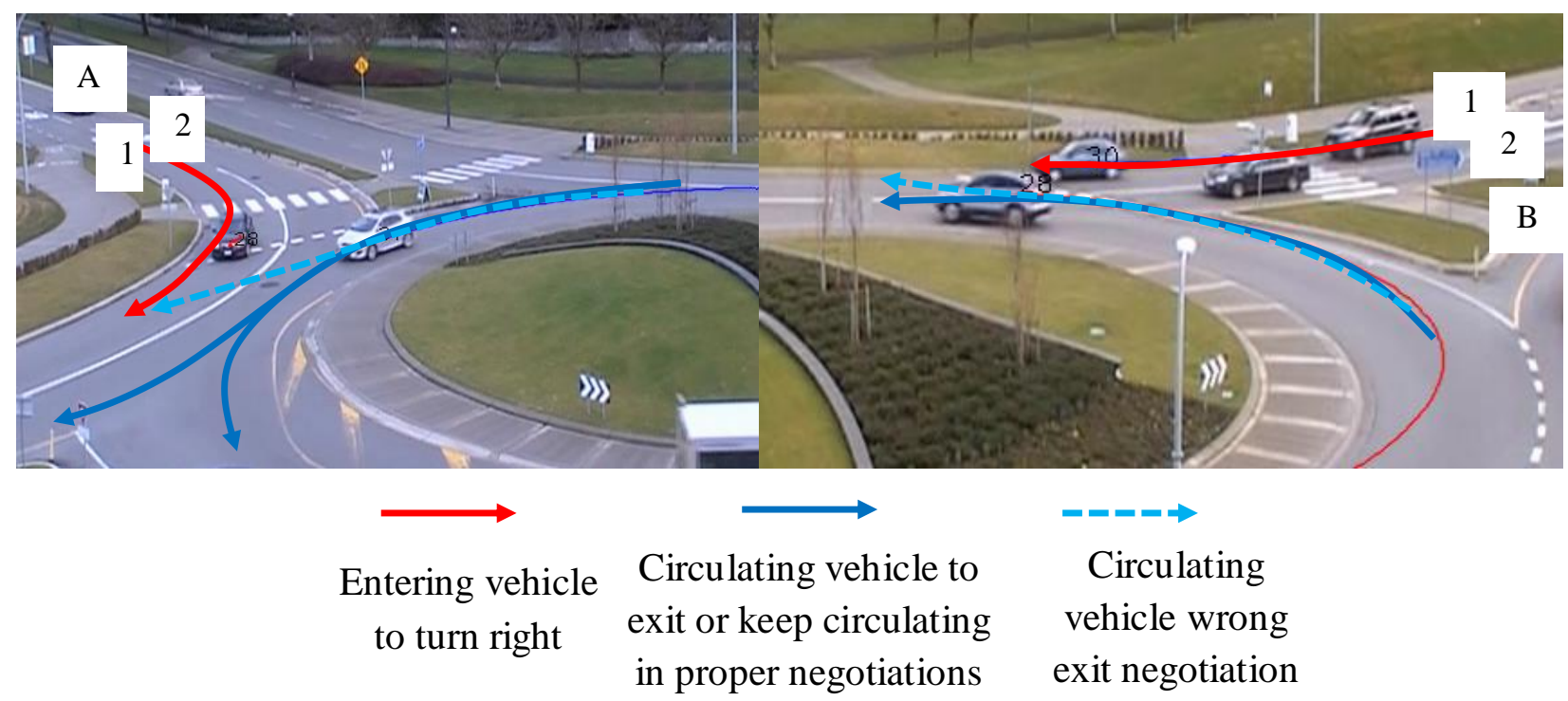

Figure 4.13 Entering-circulating conflicts and vehicles' behaviour on approaches $A$ and $B$.

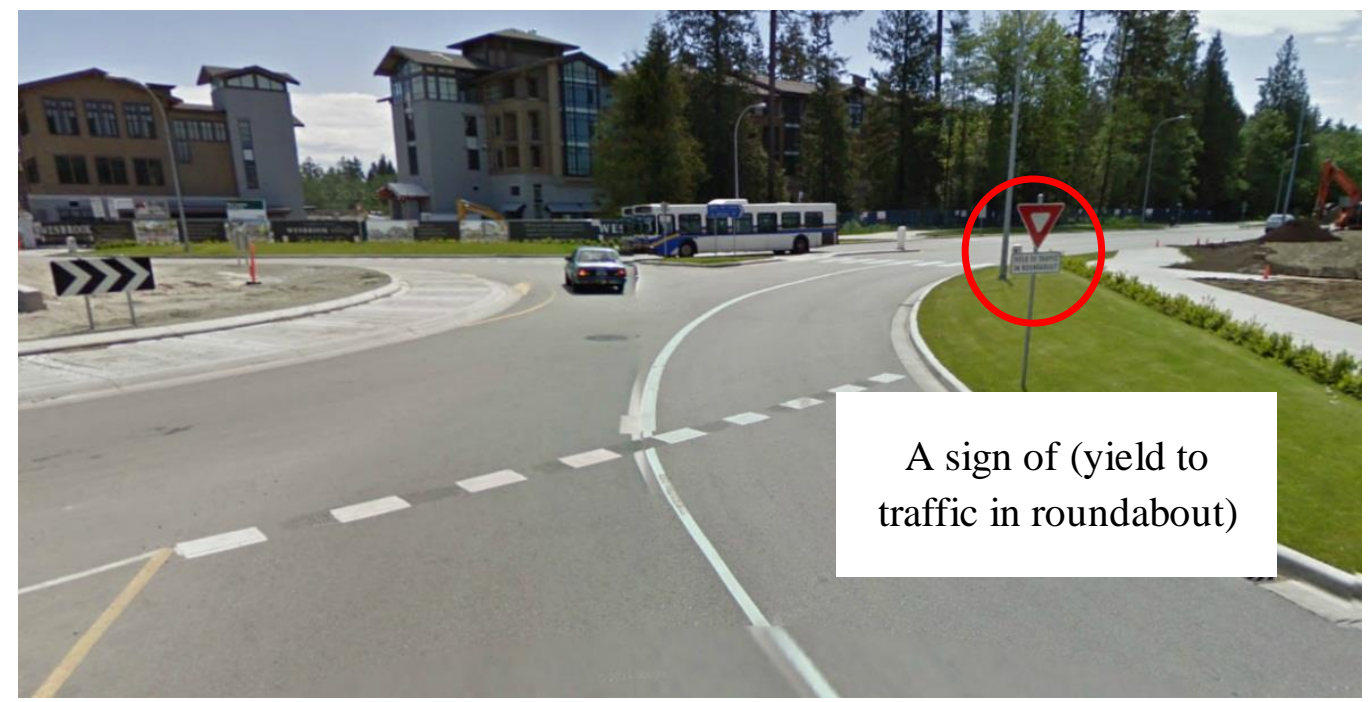

Figure 4.14 A Yield sign of traffic in roundabout when entering vehicles from approach $A$. 
- As shown in Figure 4.15, entering vehicles from lane 2 were in some cases in conflict with circulating vehicles because of hesitation in gap acceptance.

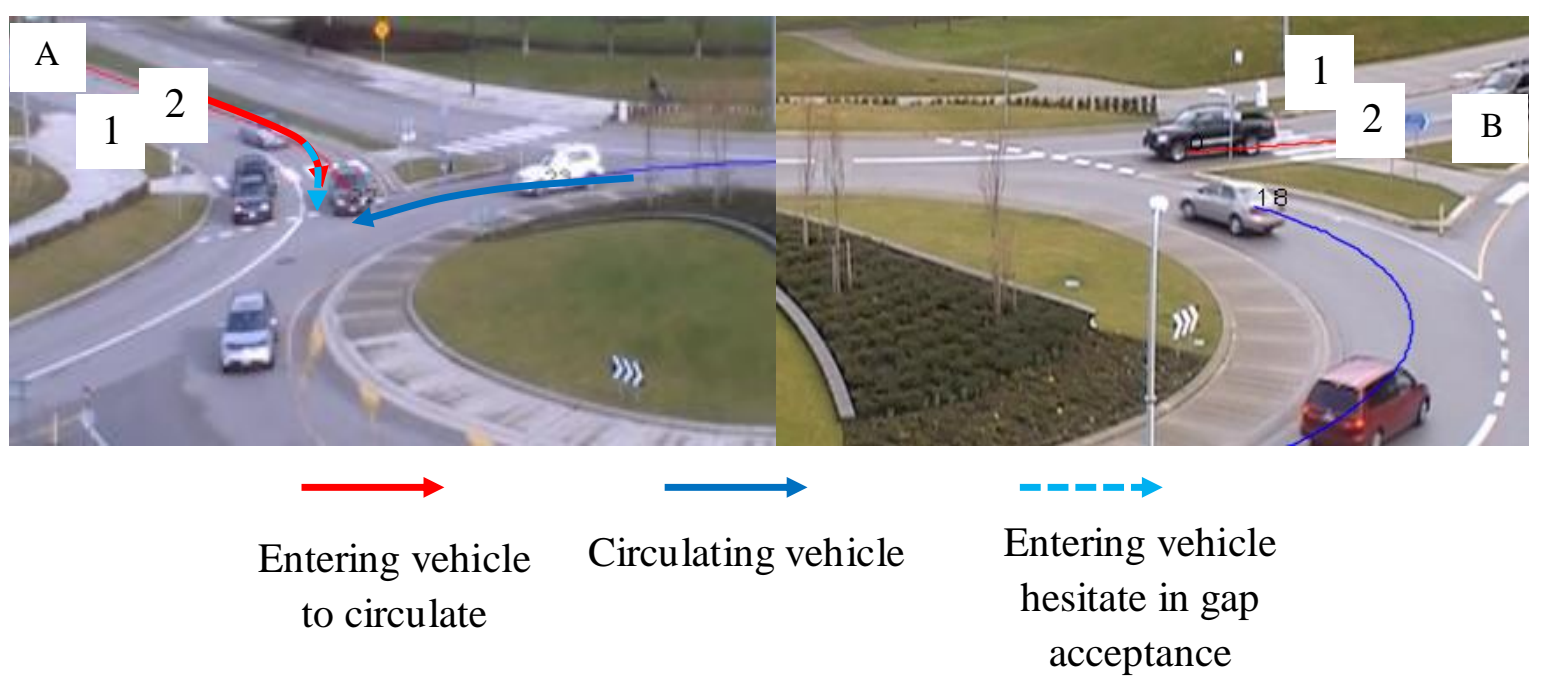

Figure 4.15 Entering-vehicle conflicts and vehicles' behaviour in gap acceptance on approach A and $B$. 
- On approach D, a similar behaviour of vehicles hesitating while entering was noticed. As shown in Figure 4.16, vehicles entering from lane 1 hesitated when entering the roundabout. Although the number of conflicts is approximately 17, drivers were confused when entering the roundabout. In this particular conflict, both conflicting vehicles had to stop in the middle of the circulating lanes as they were confused about who should yield for the other. This occurred because the circulating black vehicle was very close to the roundabout island behaving as it will continue to circulate. The white vehicle was also to blame as it should have yielded for the circulating vehicle to clear the lane before entering the roundabout.

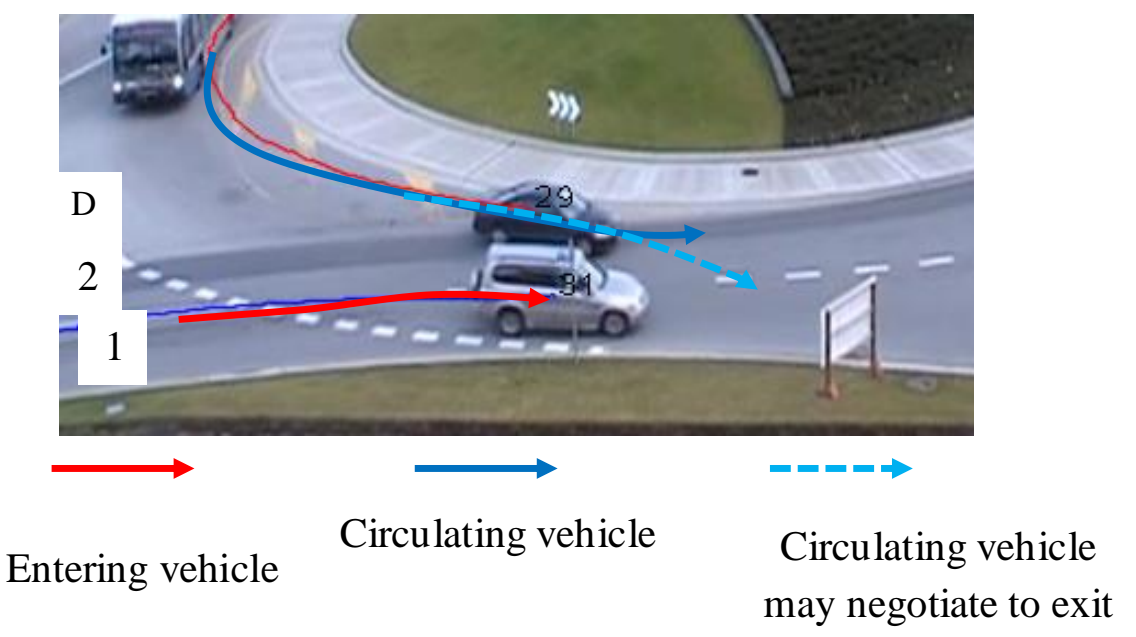

Figure 4.16 An entering-circulating conflict and vehicles' behaviour on approach $D$.

- As shown with the conflict points in Figure 4.9, entering-circulating conflict points on approach $\mathrm{C}$ are concentrated between entering vehicles and circulating vehicles at lane 1. Conflicts between entering vehicles and circulating vehicles in lane 1 were not very severe. As shown in Figure 4.17, entering vehicles are usually aware of circulating vehicles on lane 1 and they yield for them. The confusion of wrong negotiations does not 
appear in entering-circulating conflicts, but it appears in exiting-circulating conflicts near approach B as will be discussed in section 4.3.2.
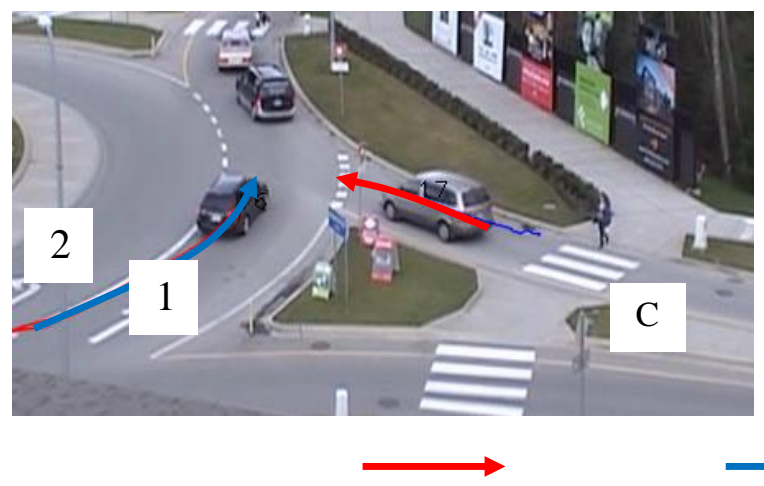

Entering vehicle coming with a

high speed
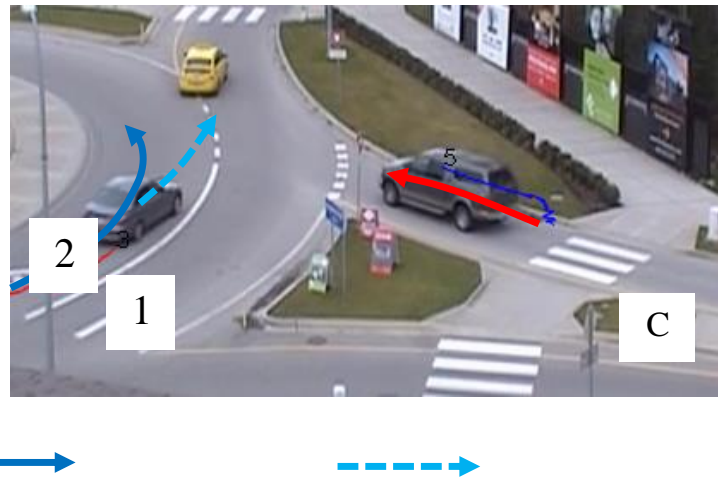

Circulating vehicle decided to exit roundabout

Figure 4.17 Entering-circulating conflicts and vehicles' behaviour on approach $\mathrm{C}$.

Figure 4.18 shows the entering-circulating conflicts TTC distribution normalized to exposure. Vehicles entering from approaches A and B have conflicts with the higher TTC distribution, and as the TTC value for conflicts increases, the frequency of conflicts per exposure is also increased. This was expected as more severe conflicts occur less frequently than less severe conflicts. 


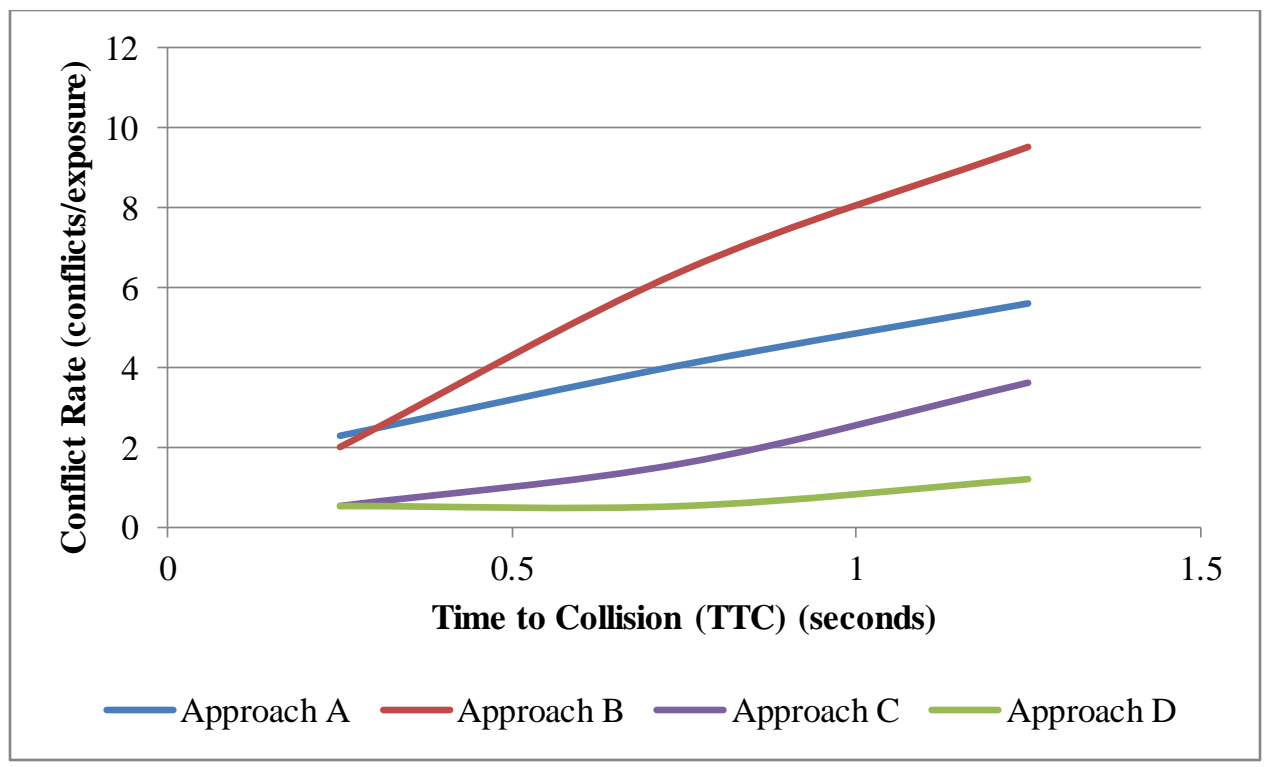

Figure 4.18 Entering-circulating conflicts TTC distribution normalized to exposure for approaches A, B, C and D.

TTC value can also be converted to a severity index using the following equation (Saunier \& Sayed, 2007):

Severity Index $(\mathrm{SI})=e^{-\left(\frac{T T C^{2}}{2 P R T^{2}}\right)}$, Eq. 4

where PRT is the perception and braking reaction time is assumed to be 2.5 seconds.

Using this equation above, the entering-circulating conflict severity can be mapped as shown in Figure 4.19. The severity distribution curve is not as smooth as the TTC distribution curve, and this is most likely because both figures include the most severe conflicts only (which have TTC values less than 1.5 seconds). The severity of these conflicts may vary depending on other variables involved in the conflict, such as the vehicles differential speed, the angle between vehicles, and the driver awareness of the potential conflict. 


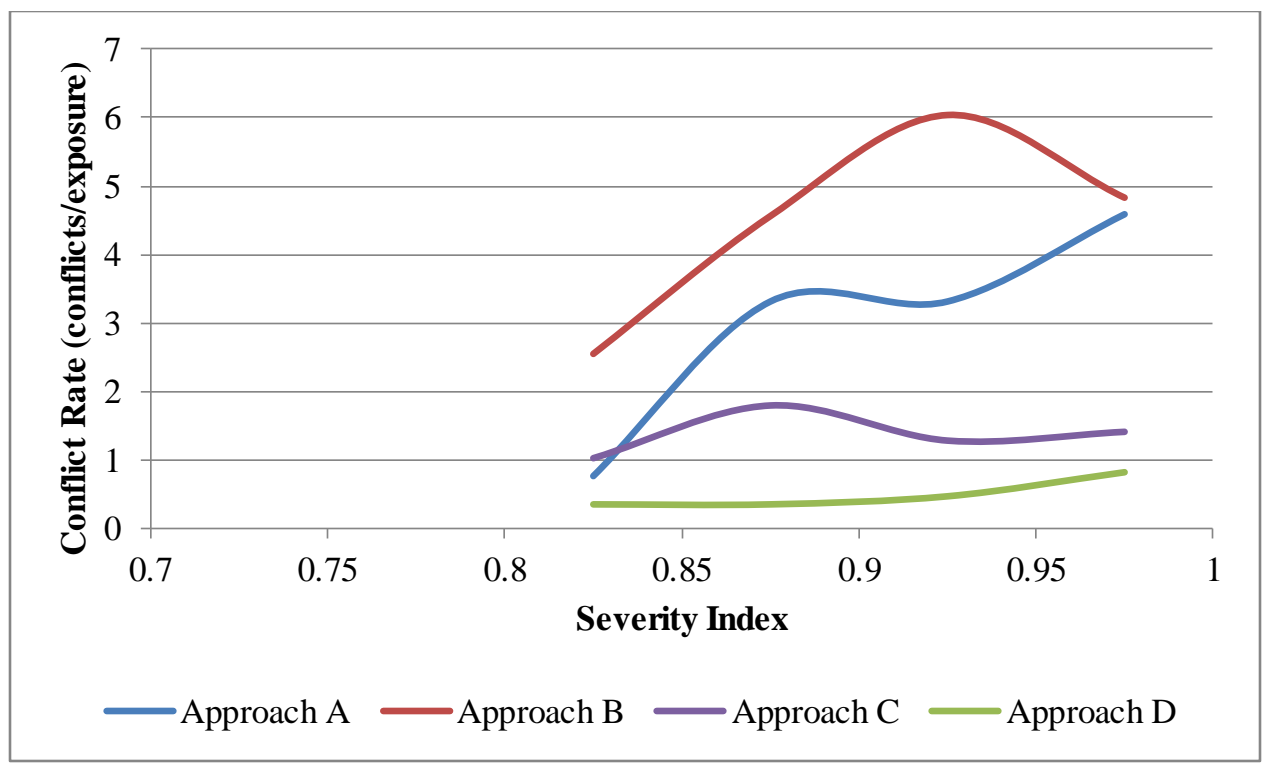

Figure 4.19 Entering-circulating conflicts severity distribution normalized to exposure for approaches A, B, C and D

\subsubsection{Exiting-circulating Conflicts}

There are approximately 62 exiting-circulating conflicts at the roundabout. Table 4.3 and Figure 4.20 show a comparison between exiting-circulating conflict rates for vehicles exiting from all approaches. The exiting-circulating conflict rates for approaches A, B, C, and D, are equal to $0.539,1.92,1.63$, and 3.72 conflicts (in 1000 vehicles) per maximum theoretical events, respectively.

Table 4.3 Comparison of the exiting-circulating conflicts in approaches A, B, C and D

$\begin{array}{lllll}\text { Approaches } & \text { A } & \text { B } & \text { C } & \text { D }\end{array}$

\begin{tabular}{l|cccc|}
\hline Circulating 1 & 6166 & 6386 & 7681 & 7788 \\
Circulating 2 & 0 & 0 & 4716 & 0 \\
Exiting 1 & 3000 & 10913 & 4350 & 4000 \\
Exiting 2 & 5916 & 0 & 0 & 4360 \\
Exposure (in 1000 vehicles) & 7.415 & 8.348 & 7.343 & 8.069 \\
No. of exiting-circulating conflicts & 4 & 16 & 12 & 30 \\
Conflicts rate = conflicts/exposure & $\mathbf{0 . 5 3 9}$ & $\mathbf{1 . 9 2}$ & $\mathbf{1 . 6 3}$ & $\mathbf{3 . 7 2}$
\end{tabular}




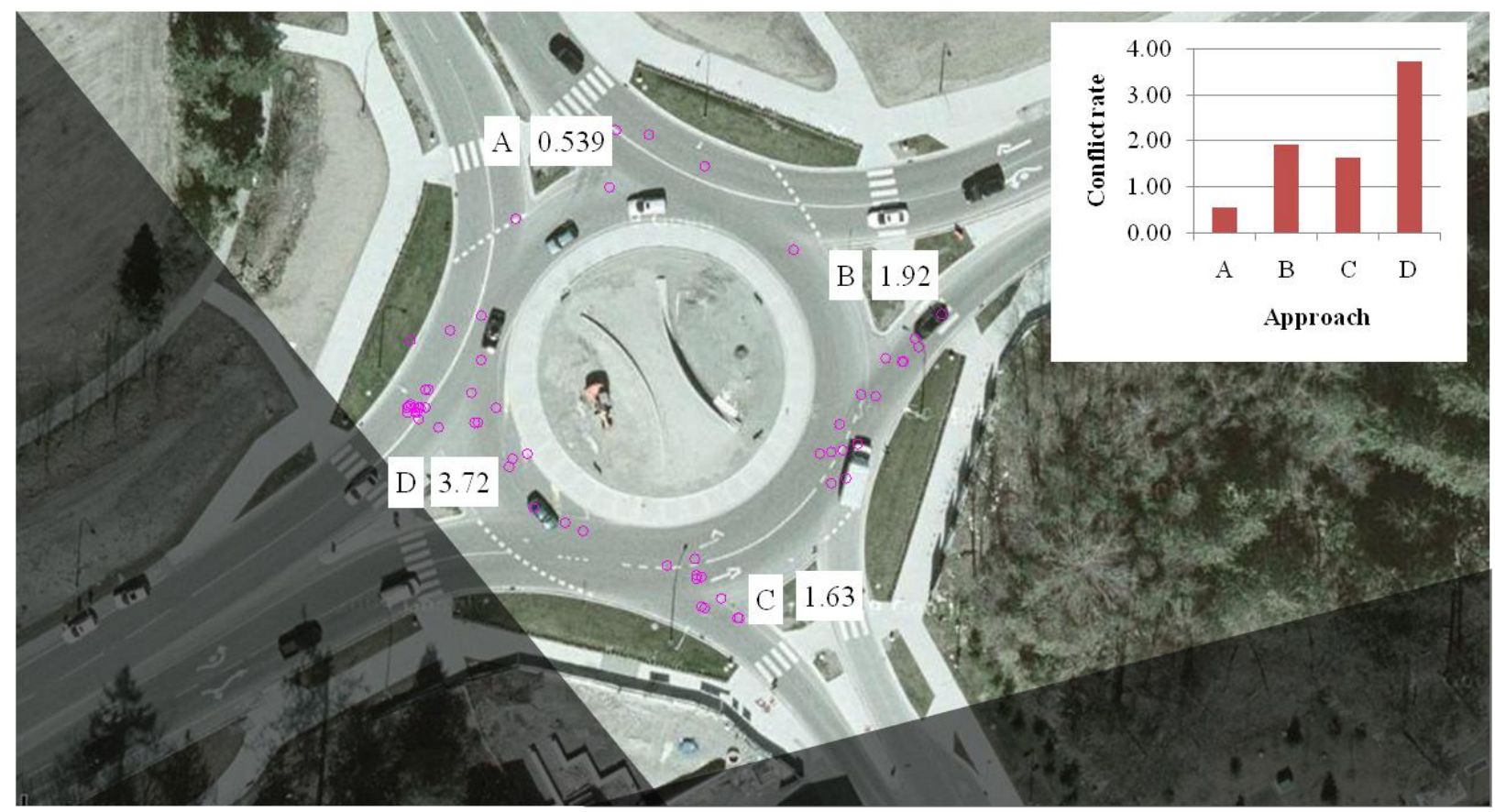

Figure 4.20 Comparison of the exiting-circulating conflicts in approaches A, B, C and D.

The exiting-circulating conflict rate for approach $\mathrm{D}$ is the highest. The conflict rates for approaches $\mathrm{B}$ and $\mathrm{C}$ are more than double the conflict rate on approach $\mathrm{A}$.

By reviewing 4, 43, 12 and 30 exiting-circulating conflicts on approaches A, B, C and D, respectively, the behaviour of the motorists and conflict causes per approach are interpreted as follows:

- On approach B, there are roughly 16 exiting-circulating conflicts. The these conflicts are classified as one of two cases:

1. Case 1: when a circulating vehicle conflicts with a following exiting vehicle (even if the circulating vehicle has just entered the roundabout or was previously circulating; 
2. Case 2: when a vehicle is exiting the roundabout and it conflicts with a following circulating vehicle (even if the exiting vehicle has just entered the roundabout or was previously circulating).

- On approach B, five conflicts are from case 1, and 11 conflicts are from case 2. Figure 4.21 shows the two cases of conflicts on approach B. Vehicles exiting the roundabout to approach B usually slow down for crossing pedestrians and this generates conflicts with the circulating vehicles following behind.
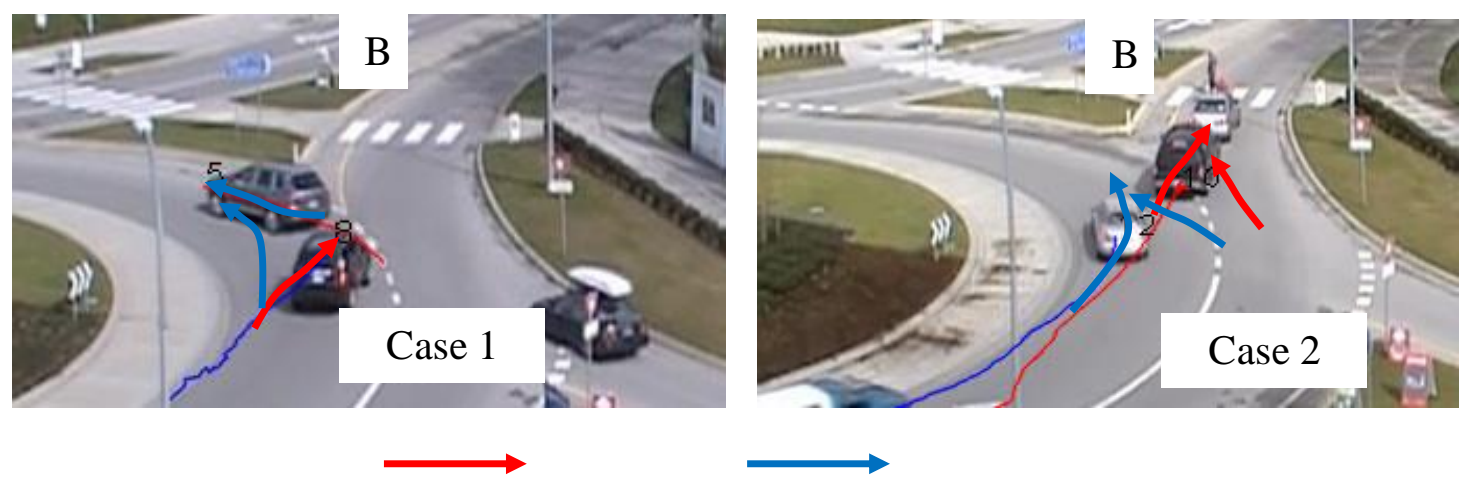

Exiting vehicle Circulating vehicle slows down

Figure 4.21 Exiting-circulating conflicts and vehicles' behaviour in a negotiation on approach B.

- On approach $\mathrm{C}$, there are 12 exiting-circulating conflicts, and all of them are from case 2. As shown in Figure 4.22, vehicles exiting the roundabout to approach $\mathrm{C}$ usually slow down for crossing pedestrians, and this generate conflicts with the circulating vehicles following behind. 


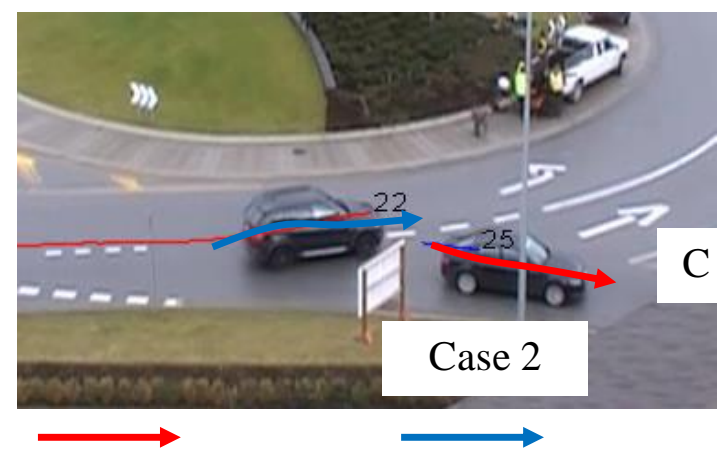

Exiting vehicle Circulating vehicle slows down

Figure 4.22 Exiting-circulating conflicts and vehicles' behaviour in negotiation on approach $\mathrm{C}$.

- On approach $\mathrm{D}$, there are 30 exiting-circulating conflicts, with eight conflicts are from case 1 and 22 from case 2. As shown in Figure 4.23, vehicles exiting the roundabout to approach D are usually slowing down for crossing pedestrians and generate conflicts with the following circulating vehicles.
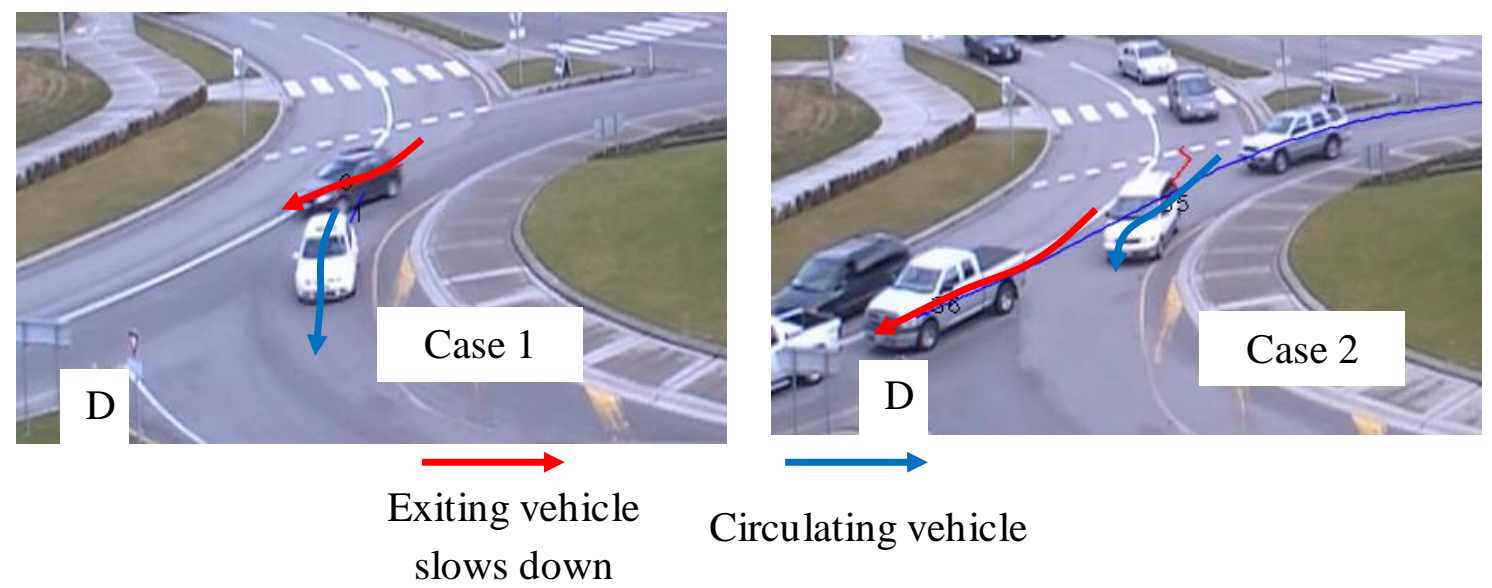

Circulating vehicle

Figure 4.23 Exiting-circulating conflicts and vehicles' behaviour in a negotiation on approach D.

Figure 4.24 shows the exiting-circulating conflicts TTC distribution normalized to exposure. The TTC distribution curve for approach D seems to be higher than the three other curves for 
approaches A, B and C. As the TTC value for conflicts increases, the frequency of conflicts per exposure is also generally increased. This was expected as more severe conflicts occur less frequently than less severe conflicts.

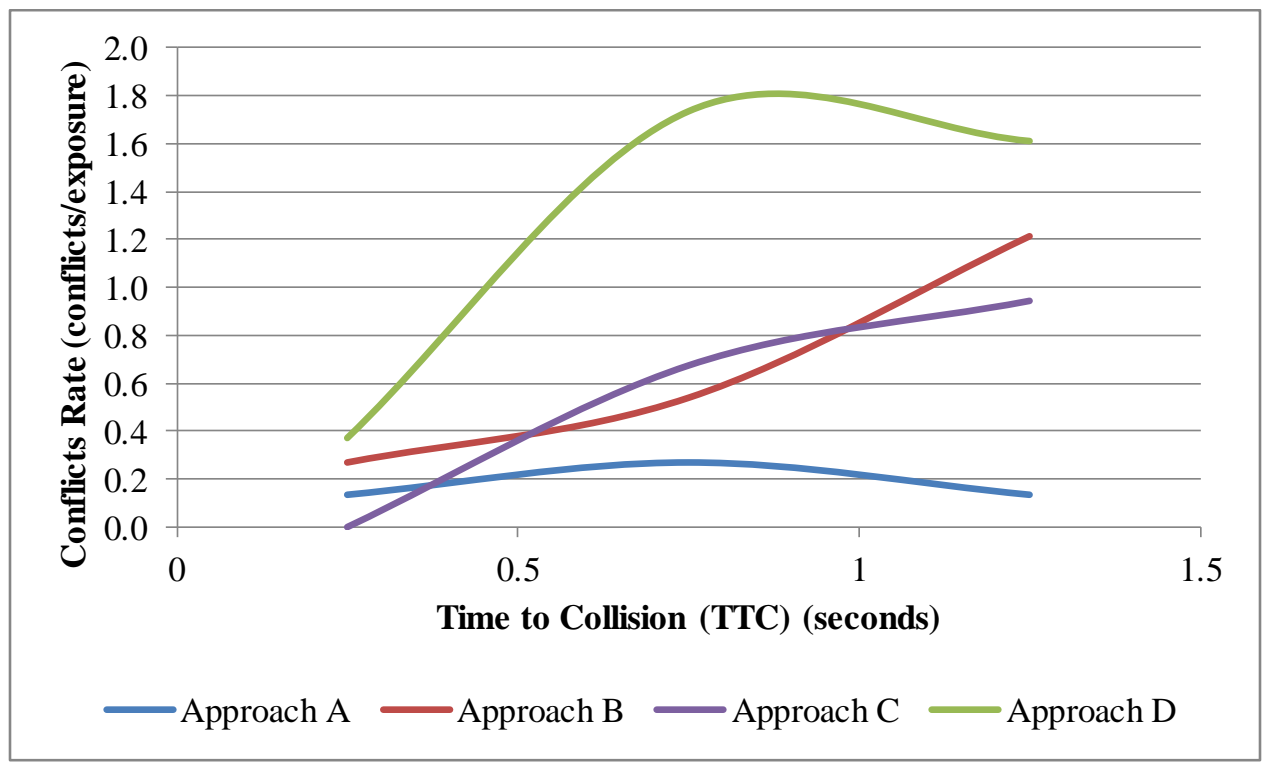

Figure 4.24 Exiting-circulating conflicts TTC distribution normalized to exposure for approaches $\mathrm{A}, \mathrm{B}, \mathrm{C}$ and $\mathrm{D}$.

Figure 4.25 shows the severity distribution for exiting-circulating conflicts. The severity distribution curve for approach $\mathrm{D}$ is the highest. The pattern of severity with the conflict rates for approaches $\mathrm{B}, \mathrm{C}$ and $\mathrm{D}$, is not clear, but this is most likely due to the severity calculation, as it does not include other variables that affect the severity of the conflict such as differential velocities, vehicle mass, and conflict angle. 


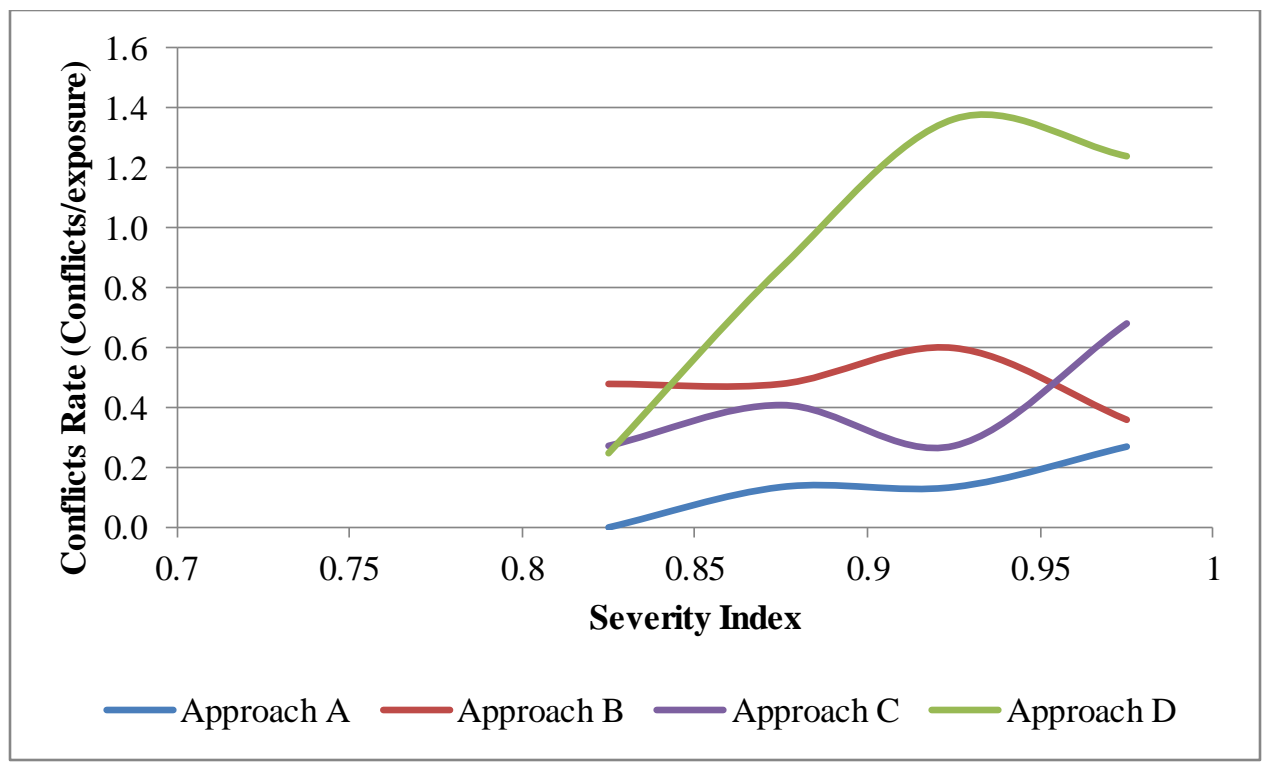

Figure 4.25 Exiting-circulating conflict severity distribution normalized to exposure for approaches A, B, C and D.

\subsubsection{Rear-end Conflicts}

A rear-end conflict can occur when a following vehicle is conflicting with a lead vehicle which can occur in different cases in the roundabout:

1. When both vehicles are entering the roundabout

2. When both vehicles are exiting the roundabout

3. When both vehicles are circulating in the roundabout

Rear-end conflicts occur because of the differential of speed between two following vehicles; if the lead vehicle is reducing its speed suddenly, or if the following vehicle is increasing its speed.

There are approximately 264 rear-end conflicts in the roundabout, and there are concentrated more in the entering lanes. Based on all rear-end conflicts in the roundabout, there are $159(60 \%)$ entering rear-end conflicts, 63 (24\%) exiting rear-end conflicts, and 42 (16\%) circulating rearend conflicts. Table 4.6 and Figure 4.26 show a comparison between the rear-end conflicts for 
entering and exiting lanes. The entering rear-end conflicts rates at approaches $\mathrm{C}$ and $\mathrm{B}$ are the highest, and the exiting rear-end conflict rates at approaches $\mathrm{C}$ and $\mathrm{B}$ are the highest. Circulating lanes between approaches B and A have a higher frequency of circulating rear-end conflicts. Approach D has only one entering rear-end conflict as the cameras did not fully capture approach D as other approaches.

Table 4.4 Comparison of rear-end conflicts in approaches A, B, C and D.

\begin{tabular}{l|cccc|} 
Approaches & A & B & C & D \\
\hline Entering lane 1 & 4314 & 3373 & 4902 & 5621 \\
Entering lane 2 & 5668 & 5323 & 0 & 3720 \\
Exposure (in 1000 vehicle) & 9.982 & 8.696 & 4.902 & 9.341 \\
No. of entering rear-end conflicts & 26 & 41 & 63 & 1 \\
Conflict rate = conflicts/exposure & $\mathbf{2 . 6 0}$ & $\mathbf{4 . 7 1}$ & $\mathbf{1 2 . 9}$ & $\mathbf{0 . 1 0 7}$ \\
Exiting lane 1 & 3000 & 10913 & 4350 & 4000 \\
Exiting lane 2 & 5916 & 0 & 0 & 4360 \\
Exposure (in 1000 vehicle) & 8.916 & 10.913 & 4.350 & 8.360 \\
No. of exiting rear-end conflicts & 4 & 37 & 17 & 4 \\
Conflict rate = conflicts/exposure & $\mathbf{0 . 4 5}$ & $\mathbf{3 . 3 9}$ & $\mathbf{3 . 9 1}$ & $\mathbf{0 . 4 8}$ \\
\cline { 2 - 6 }
\end{tabular}




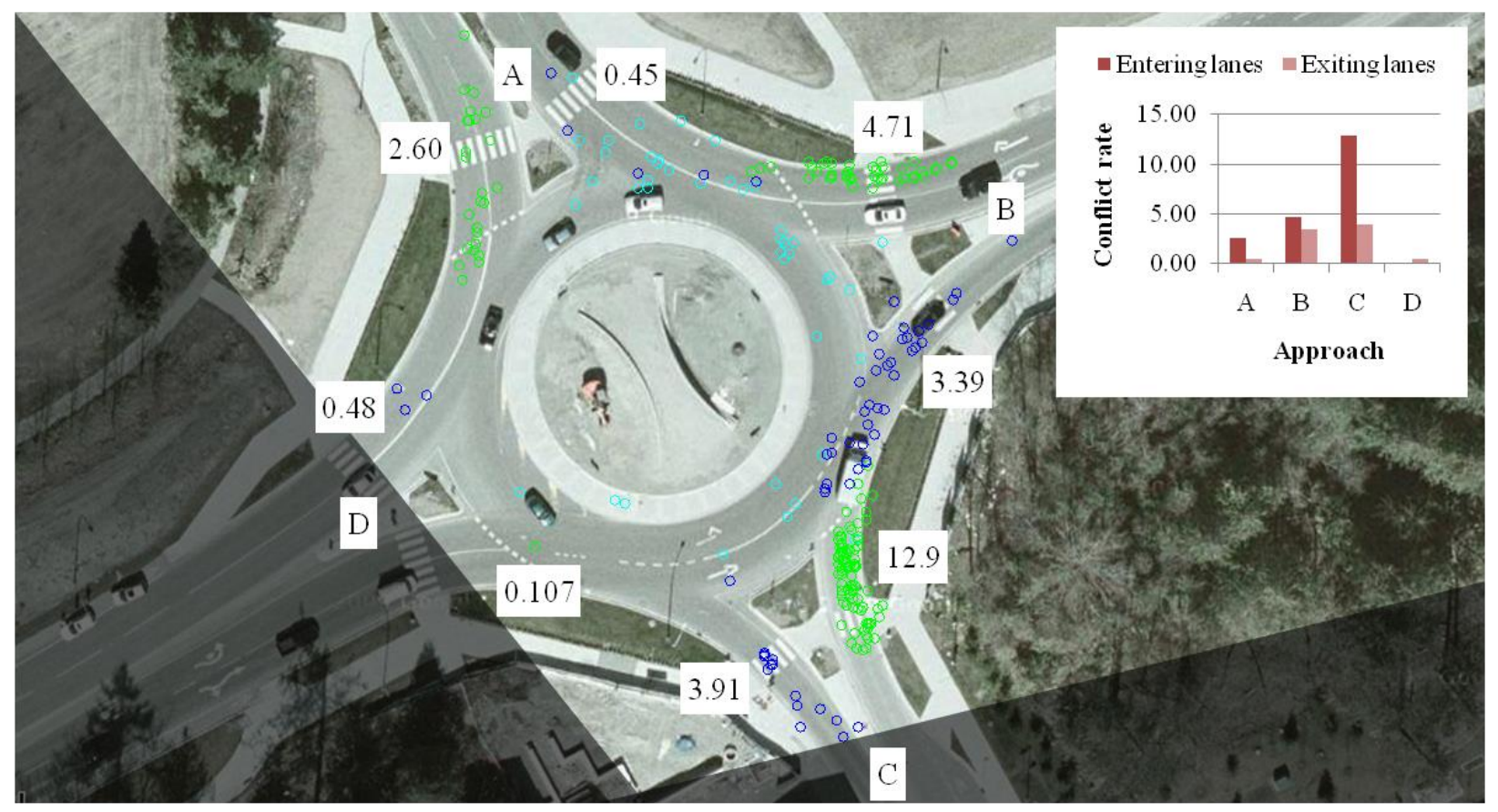

Figure 4.26 Comparison between rear-end conflicts in approaches A, B, C and D.

The entering and exiting rear-end conflict rates for approach $\mathrm{C}$ are 12.9 and 3.91, respectively. The conflict rate on approach $\mathrm{C}$ is more than double the conflict rates of all other approaches.

By reviewing 159 entering rear-end conflicts, 63 exiting rear-end conflicts and 42 circulating rear-end conflicts on all approaches, the behaviour of motorists and conflict causes per approach are interpreted as follows: 
- As shown in Figure 4.27, vehicles that enter the roundabout from all approaches usually slow down for crossing pedestrians, circulating vehicles, or previously entered vehicles. All conditions lead to generate conflicts with the following vehicles entering the roundabout.
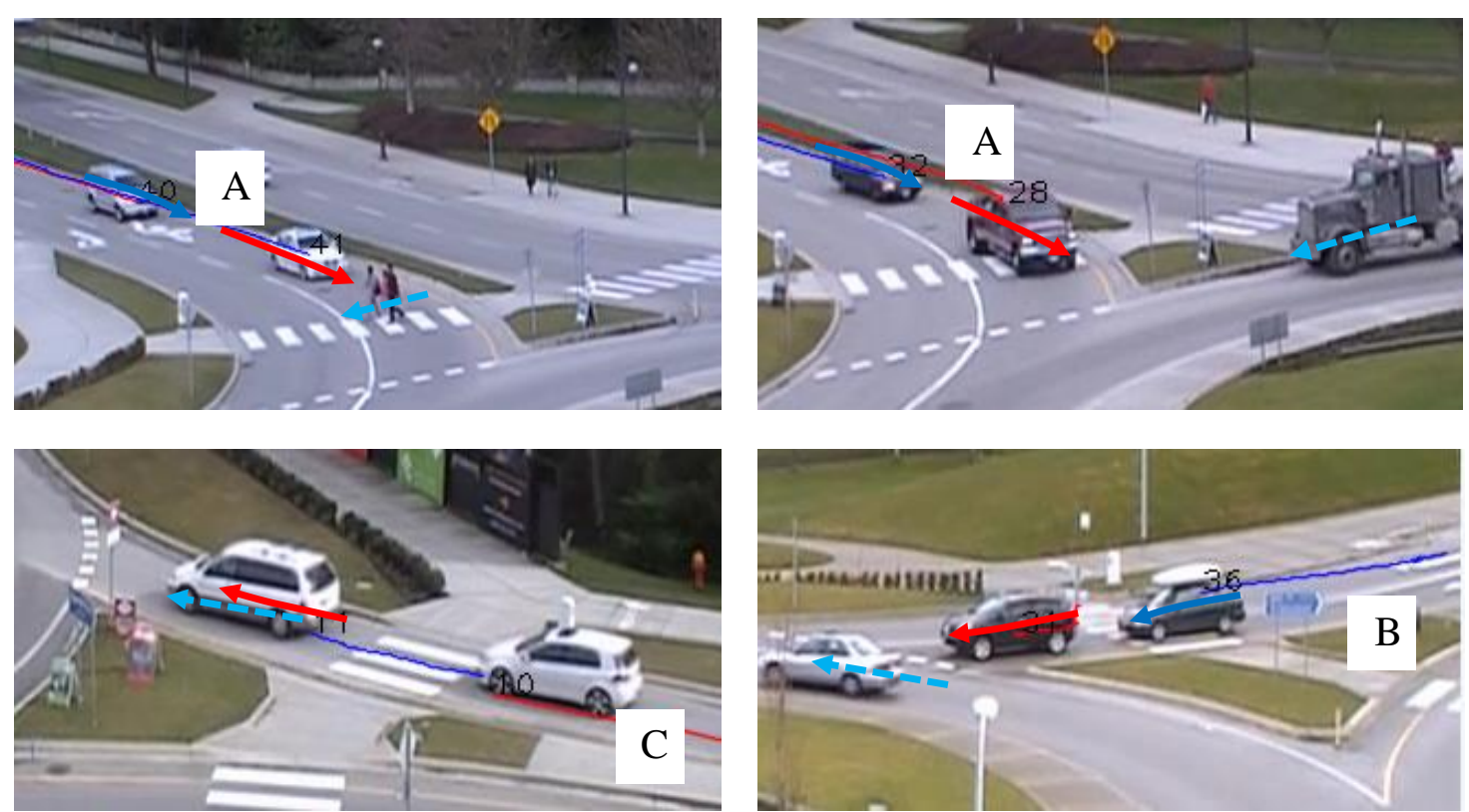

Entering vehicle
slows down

Following entering

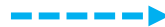
vehicle

Road user causes entering vehicle to slow down

Figure 4.27 Entering rear-end conflicts and vehicles' behaviour in all approaches.

- As shown in Figure 4.28, vehicles that exit the roundabout from all approaches usually slow down for crossing pedestrians, or previously exiting vehicles. All these conditions lead to generate conflicts with the following exiting vehicles.

- As shown in Figure 4.28 and Figure 4.29, some exiting rear-end conflicts on approach C are generated because some exiting vehicles turn right just past the crosswalk. 

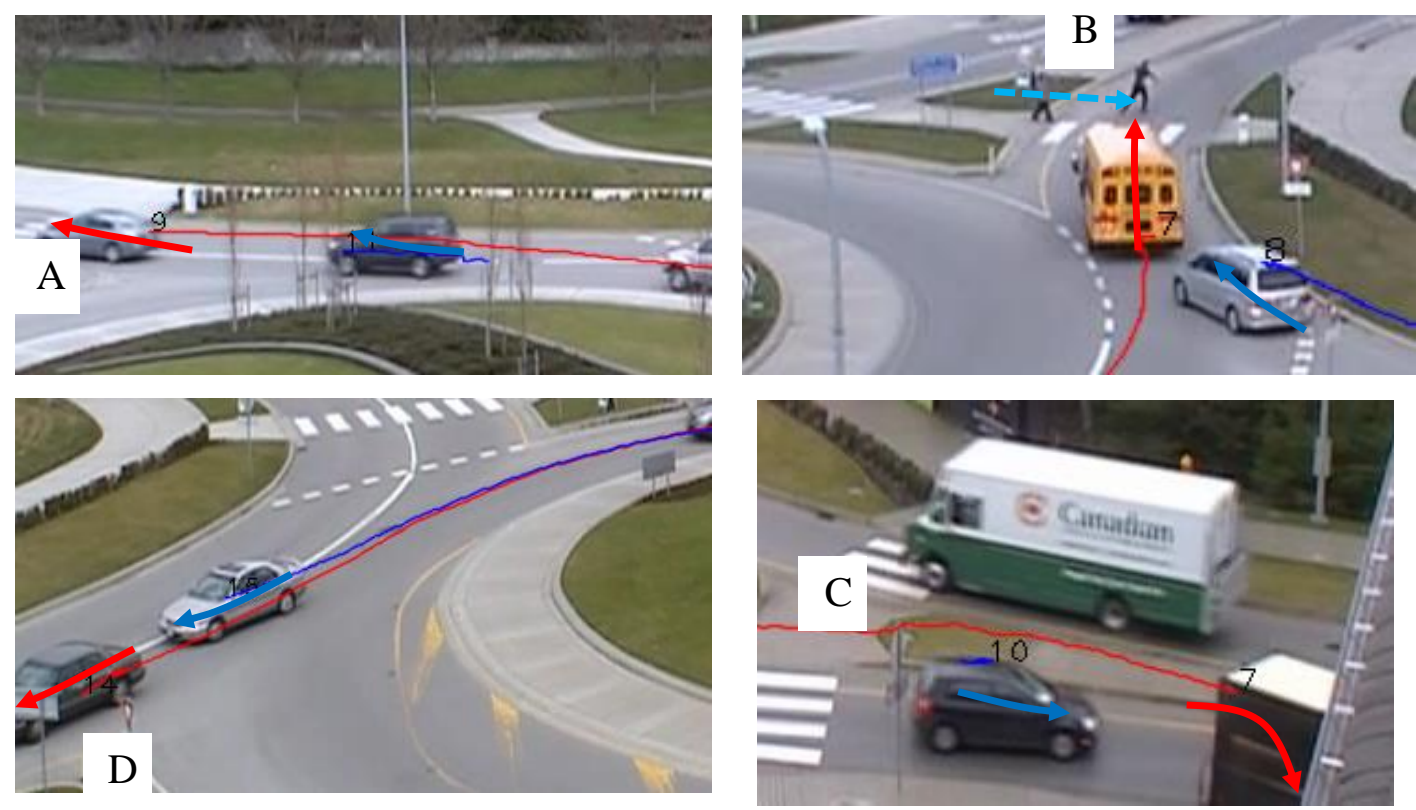

Exiting vehicle

Following exiting vehicle
Road user causes exiting vehicle to slow down

Figure 4.28 Entering rear-end conflicts and vehicles' behaviour in all approaches.

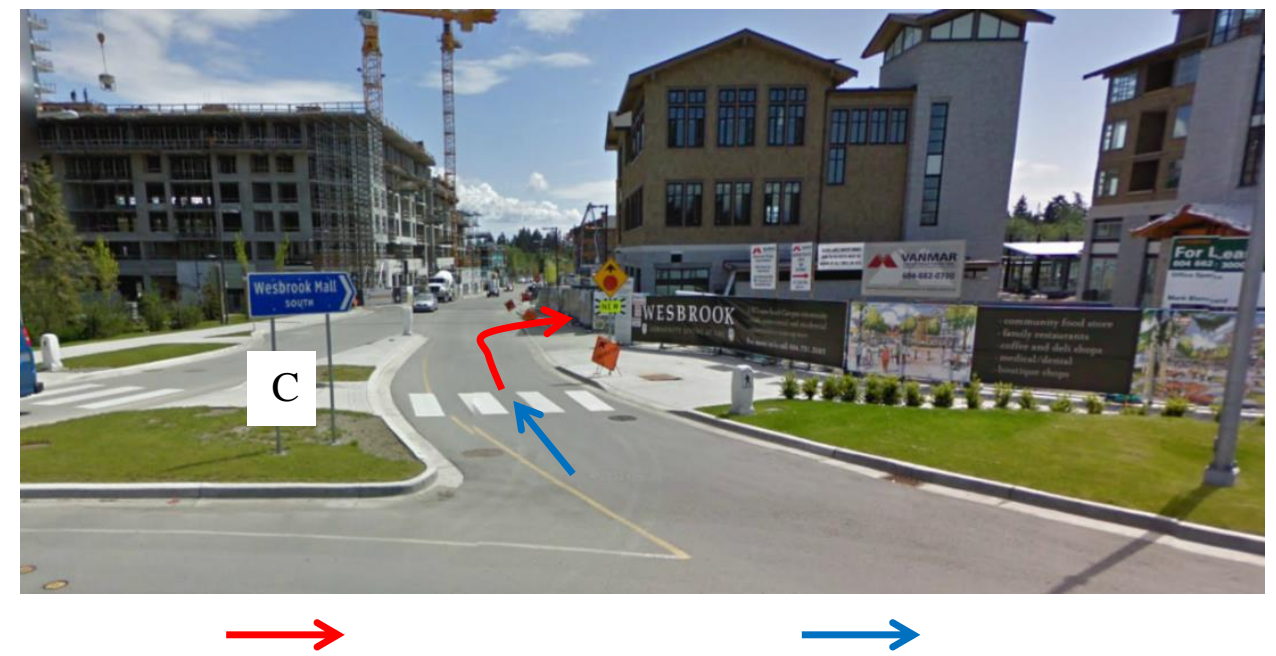

Exiting vehicle is turning Following exiting vehicle right just after the crosswalk

Figure 4.29 Exiting vehicles turning right past the pedestrian crosswalk on approach $C$. 


\subsubsection{Sideswipe conflicts}

Sideswipe conflicts usually occur in circulating and turning lanes. As shown in Figure 4.30, there were approximately 25 sideswipe conflicts at the roundabout. A total of 21 conflicts (84\%) occurred in the circulating lanes between approaches A and B.

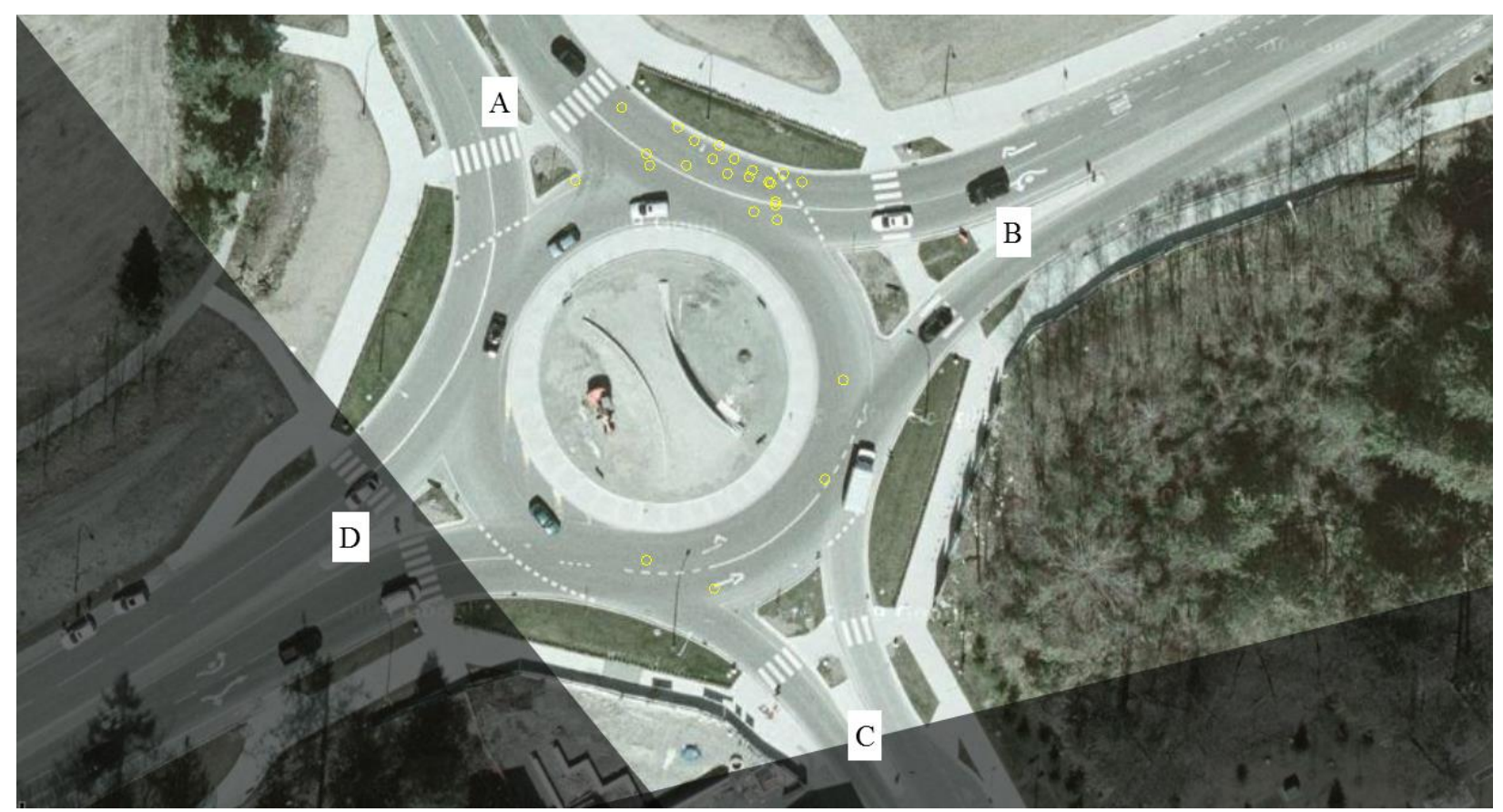

Figure 4.30 Sideswipe conflict points

By reviewing the 25 sideswipe conflicts, the behaviour of motorists and conflict causes per approach are interpreted as follows:

- Most of the sideswipe conflicts are generated between approaches A and B, and are among circulating vehicles and entering vehicles which just started circulating at a high velocity (Figure 4.31). 


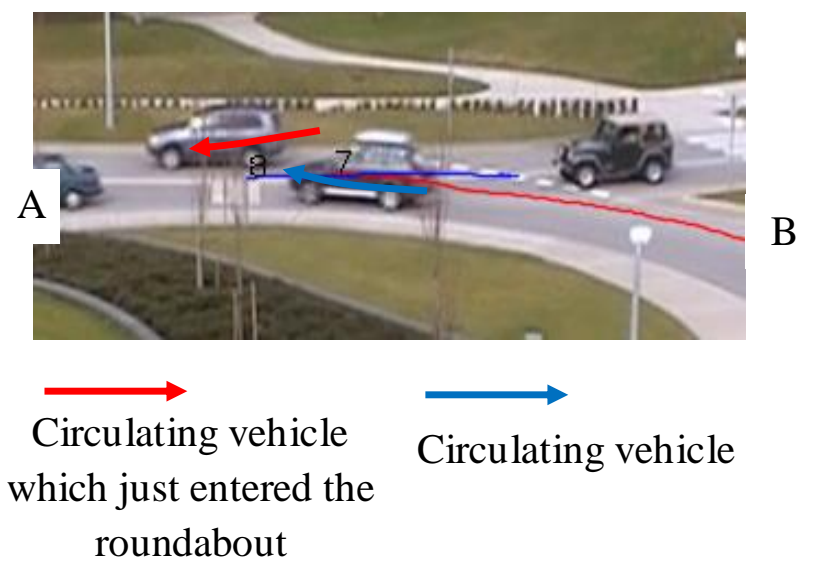

Figure 4.31 Example of sideswipe conflicts

\subsubsection{Violations and wrong negotiations of vehicle-vehicle interactions}

Studying violations and wrong negotiations of vehicle interactions in the roundabout helps to determine if there are any safety issues with the roundabout and if there is a lack of understanding of how to use a roundabout and its right of way rules.

In vehicle interactions, a violation of a vehicle entering the roundabout from approach $\mathrm{C}$ has been noticed. It is a type of violation that turned out to be a conflict. Figure 4.32 shows the violation and the conflict generated by the vehicle. 

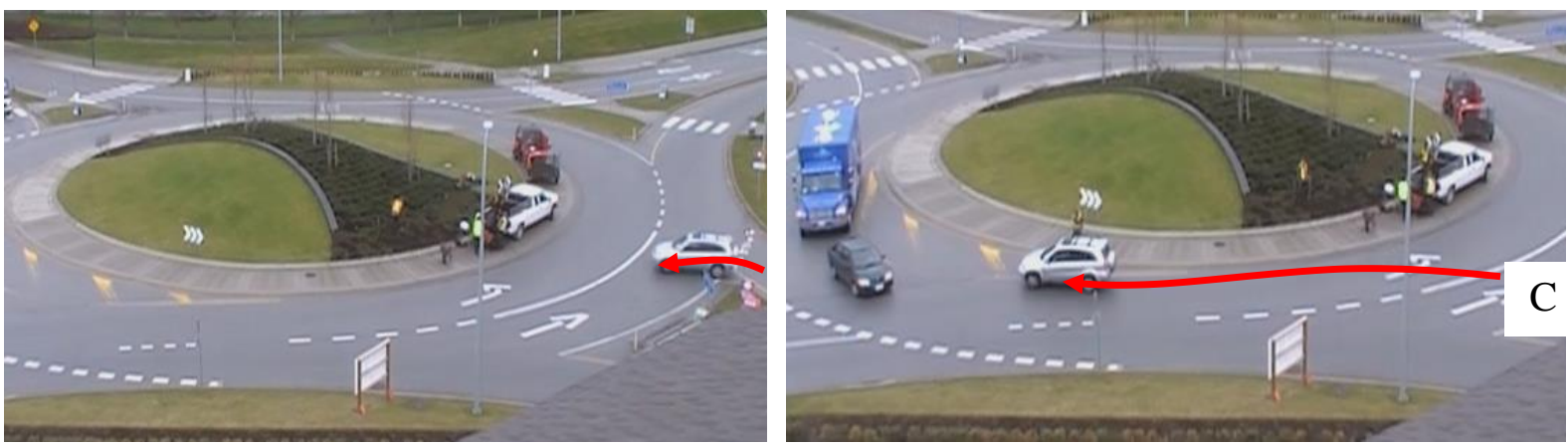

Figure 4.32 Violation of a vehicle entering from approach $\mathrm{C}$ and turning left in error.

Another wrong negotiation occurred when a circulating vehicle misunderstood the right of way rules for roundabouts, as it assumed that it had to yield to entering vehicles, which led to both entering and circulating vehicles stopping for each other. This type of behaviour was noticed on two occasions, and this wrong negotiation did not generate a conflict between entering and circulating vehicles, but it resulted in a rear-end conflict between the circulating vehicle and the following circulating vehicle.
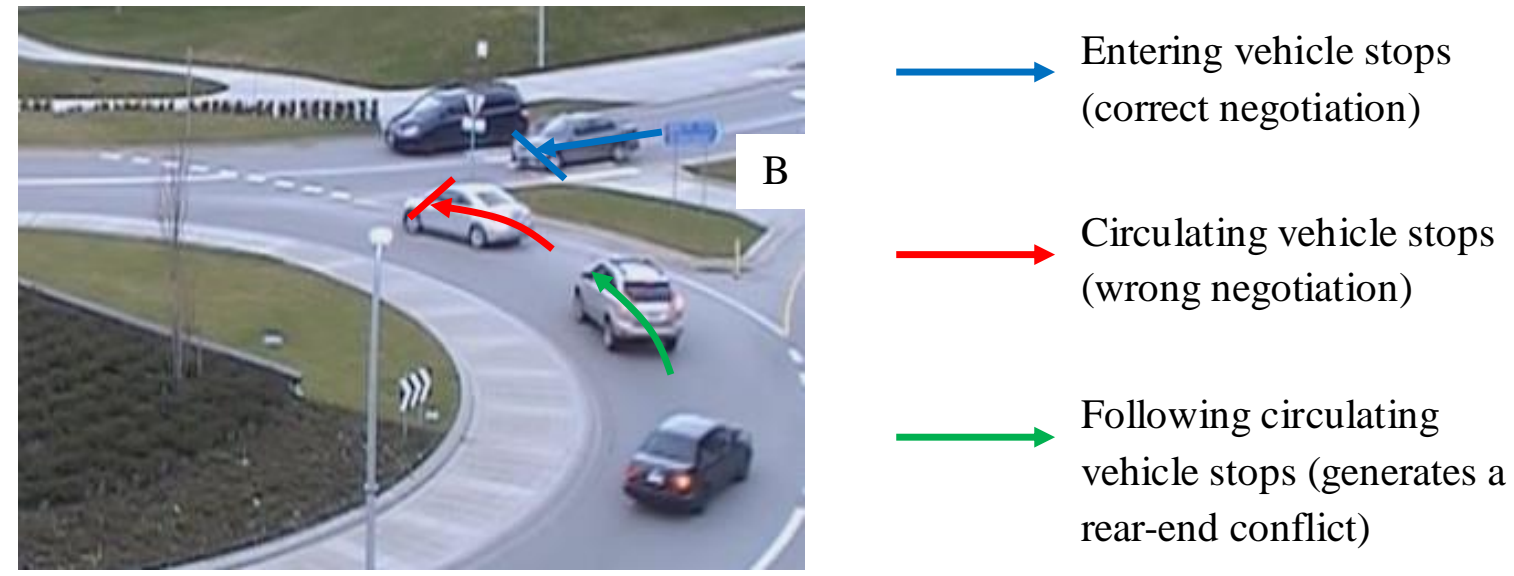

Figure 4.33 A circulating vehicle yields in error for an entering vehicle.

A similar wrong negotiation has been seen on approach D as shown in Figure 4.34. 

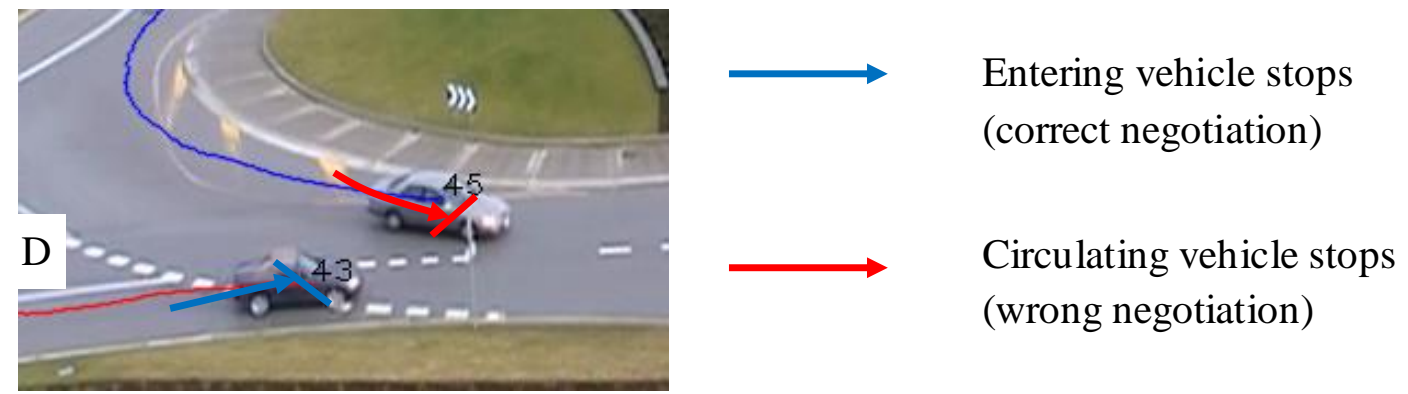

Figure 4.34 A circulating vehicle yields in error for an entering vehicle mistake

\subsubsection{Overall Assessment of All Vehicle-vehicle Conflicts:}

Across all vehicle-vehicle conflicts, there were some commonalities and key differences between conflicts' types. Both exiting-circulating and rear-end vehicle-vehicle conflicts occurred because of the interaction of vehicles with crossing pedestrians at cross walks. It is most likely occurred because drivers approaching and exiting the roundabout were not fully aware of the pedestrian while crossing. On the other hand, entering-circulating conflicts were not related to pedestrian crossing but were mainly because of drivers' yielding behavior and expectation of wrong negotiation for both entering and circulating vehicles.

The conflict rates per exposure were calculated for entering-circulating conflicts, exitingcirculating conflicts, and rear-end conflicts for each approach at the roundabout. A weighted average of the conflict rate was calculated for each conflict type for comparison as shown in Table 4.5. 
Table 4.5 Weighted average values for conflict rates across conflict's types

\begin{tabular}{|c|cccc|} 
Conflict Type & $\begin{array}{c}\text { Entering- } \\
\text { circulating }\end{array}$ & $\begin{array}{c}\text { Exiting- } \\
\text { circulating }\end{array}$ & $\begin{array}{c}\text { Rear End } \\
\text { (entering) }\end{array}$ & $\begin{array}{c}\text { Rear Eng } \\
\text { (exiting) }\end{array}$ \\
\hline $\begin{array}{c}\text { Conflict rate } \\
\text { (conflicts/exposure) }\end{array}$ & 9.11 & 1.99 & 3.98 & 1.9
\end{tabular}

The entering-circulating conflict rate was the highest among all conflict types. This result is quite expected in roundabouts compared to other road segments or intersections.

\subsection{Vehicle-pedestrian Conflicts}

\subsubsection{Conflicts at Pedestrian Crosswalks}

A vehicle-pedestrian conflict is defined as an interaction between a vehicle and a pedestrian which requires that one or both of them have to react or stop suddenly to avoid a collision. Figure 4.35 shows an example of a vehicle-pedestrian conflict.

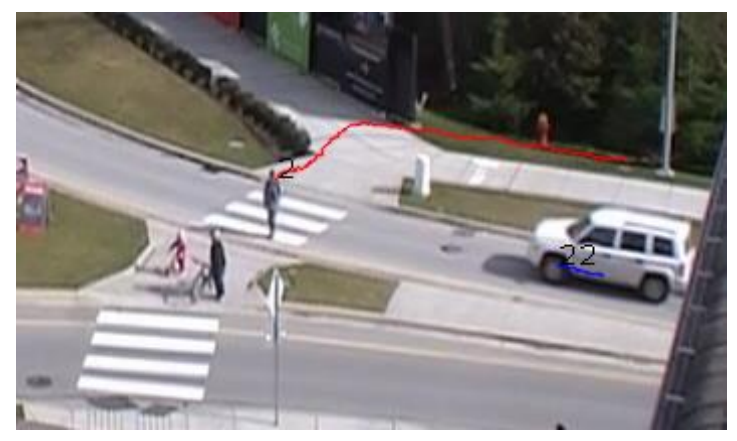

Figure 4.35 Example of a vehicle-pedestrian conflict.

On approaches A, B, and C, there were approximately 147 vehicle-pedestrian conflicts in the two-day analysis. These conflicts recorded a TTC value of less than 1.5 seconds (45 frames). Figure 4.36 shows the frequency of vehicle-pedestrian conflicts at entry and exit lanes for approaches $\mathrm{A}, \mathrm{B}$, and $\mathrm{C}$. 


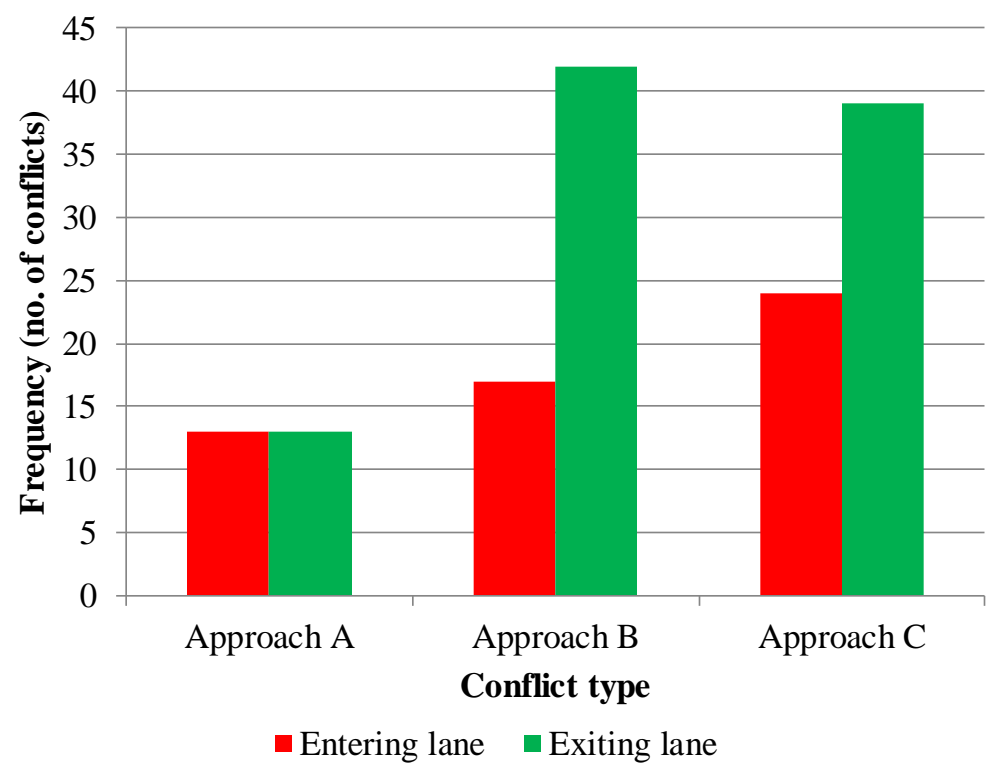

Figure 4.36 Frequency of vehicle-pedestrian conflicts at the entry and exit lanes of approaches A, B and $\mathrm{C}$.

To account for different exposure levels, Table 4.6 and Figure 4.37 show a comparison between the conflicts per exposure across approaches at entry and exit lanes. The conflict points occurring on entering and exiting lanes are indicated by a red and green colour, respectively. The conflicts per exposure rates at entry lanes for approaches A, B and C, are 5.43, 5.25, and 10.79 conflicts per maximum theoretical conflicts, respectively. However, the conflicts per exposure rates at exit lanes for approaches A, B and C, are 5.74, 11.57, and 18.62 conflicts per maximum theoretical conflicts, respectively. 
Table 4.6 Comparison of vehicle-pedestrian conflict rates at the entry and exit lanes of approaches $A, B$, and $C$

\begin{tabular}{l|ccc|} 
Approaches & A & B & C \\
\hline Entering lane 1 & 4314 & 3373 & 4902 \\
Entering lane 2 & 5668 & 5323 & 0 \\
Crossing pedestrians from entry lane & 307 & 607 & 544 \\
Crossing pedestrians from exit lane & 268 & 600 & 465 \\
Exposure (in 1000 vehicles) & 2.396 & 3.240 & 2.224 \\
No. of entering-veh-ped conflicts & 13 & 17 & 24 \\
Conflict rate = conflicts/exposure & $\mathbf{5 . 4 3}$ & $\mathbf{5 . 2 5}$ & $\mathbf{1 0 . 7 9}$ \\
Exiting lane 1 & 3000 & 10913 & 4350 \\
Exiting lane 2 & 5916 & 0 & 0 \\
Crossing pedestrians from entry lane & 307 & 607 & 544 \\
Crossing pedestrians from exit lane & 268 & 600 & 465 \\
Exposure (in 1000 vehicles) & 2.264 & 3.629 & 2.095 \\
No. of exiting-veh-ped conflicts & 13 & 42 & 39 \\
Conflict rate = conflicts/exposure & $\mathbf{5 . 7 4}$ & $\mathbf{1 1 . 5 7}$ & $\mathbf{1 8 . 6 2}$ \\
\cline { 2 - 4 } & & &
\end{tabular}




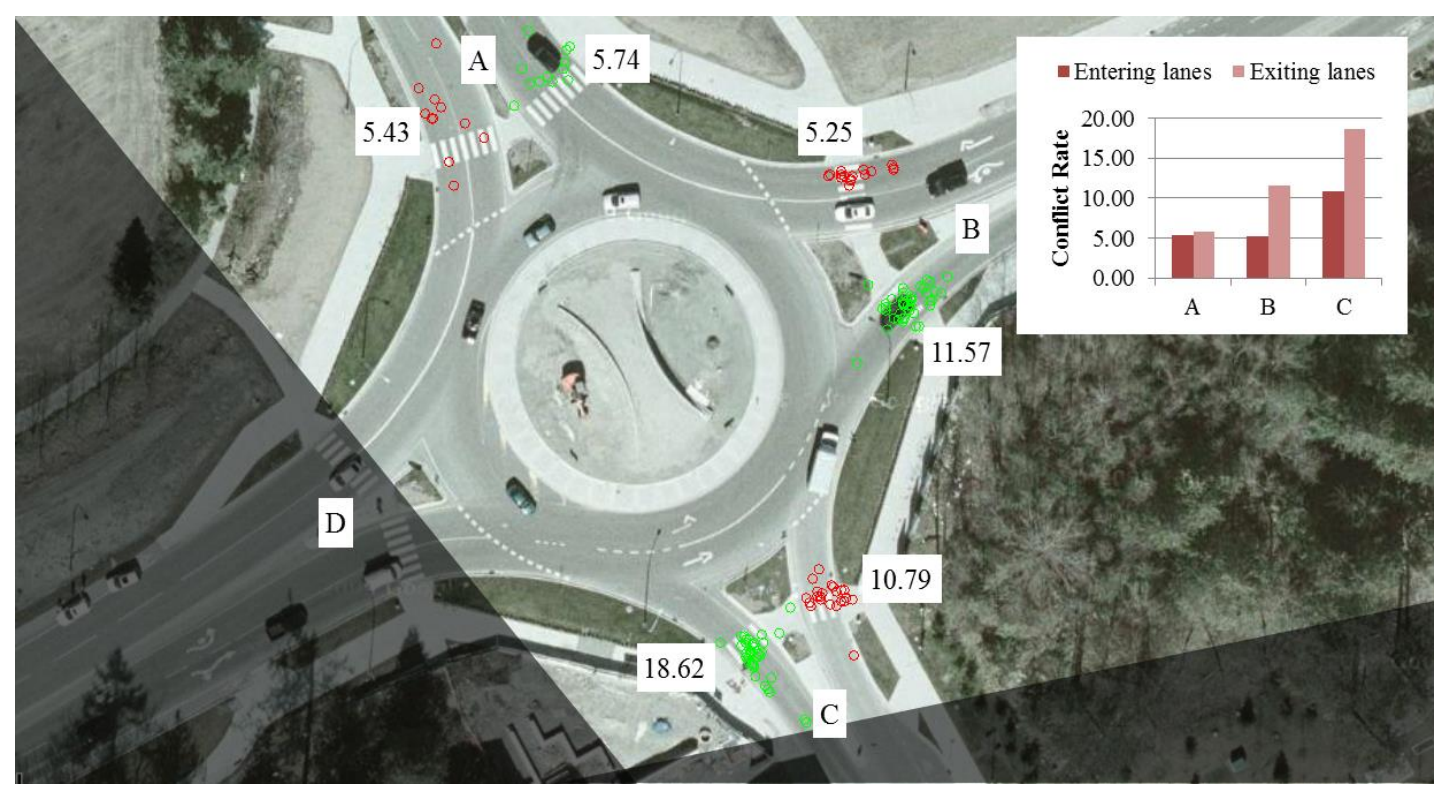

Figure 4.37 Comparison of vehicle-pedestrian conflict rates at entry and exit lanes of approaches A, $\mathrm{B}$, and $\mathrm{C}$.

Exit lanes generally have more pedestrian conflicts than at the entering lanes. A study in the United States concluded that the exiting lanes appear to place crossing pedestrians at a greater risk than entry lanes (Harkey \& Carter, 2006). Conflict rates in exiting lanes were 11.57 and 18.62 conflicts per exposure at approaches B and C, respectively.

\subsubsection{Yielding Behaviour of Motorists}

The yielding behaviour of motorists in vehicle-pedestrian conflicts can be analyzed on the entry and exit lanes on all approaches. The yielding behaviour is categorized based on NCHRP Report 572 (Rodegerdts, et al., 2007) as follows:

- Active yield: The motorist slowed or stopped only because of a crossing or waiting pedestrian. 
- Passive yield: The motorist slowed or stopped, not because of a crossing or waiting pedestrian, but for other reasons such as the queue of vehicles waiting to enter or exit the roundabout or already stopped for a previous pedestrian.

- Did not yield: The motorist did not yield for the crossing or waiting pedestrian.

For the 147 vehicle-pedestrian conflicts, the yielding behaviour is analyzed on both entry and exit lanes. Figure 4.38 presents the yielding behaviour of motorists when pedestrians are crossing. There were $76 \%$ and $45 \%$ of motorists who actively yielded for pedestrians in entry lanes and exit lanes respectively. There were $22 \%$ and $54 \%$ of motorists who did not yield for pedestrians in entry lanes and exit lanes, respectively.

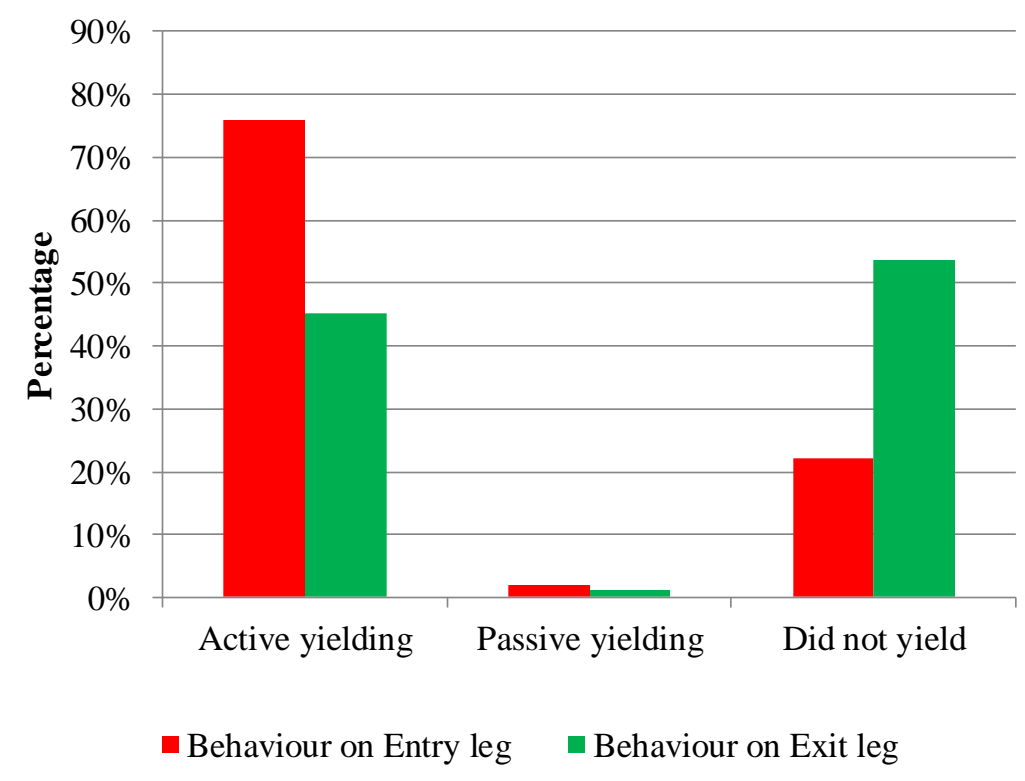

Figure 4.38 Yielding behaviour of motorists when pedestrians are crossing 


\subsubsection{Crossing Behaviour of Pedestrians}

The crossing behaviour of pedestrians in vehicle-pedestrian conflicts at entry and exit lanes can be analyzed for all approaches. The crossing behaviour is categorized based on NCHRP Study (Rodegerdts, et al., 2007) as follows:

- Normal crossing: The pedestrian starts crossing or keeps crossing while the vehicle is present without hesitating, running, or stopping.

- Hesitate: The pedestrian hesitates if he or she should stop, keep crossing, or wait on the curb when there is a vehicle present. This usually occurs when the pedestrian is not fully aware of the vehicle.

- Run: The pedestrian starts or keeps running before or while crossing.

Pedestrian behaviour is analyzed on both entry and exit lanes on 147 vehicle-pedestrian conflicts.

Figure 4.39 presents the crossing behaviour of pedestrians when a vehicle is present. Approximately $80 \%$ of pedestrians crossed normally in entry and exit lanes, however, $7 \%$ and $13 \%$ hesitated while crossing in entry lanes and exit lanes, respectively. Approximately $13 \%$ and $5 \%$ of motorists did not yield to pedestrians in entry lanes and exit lanes, respectively. 


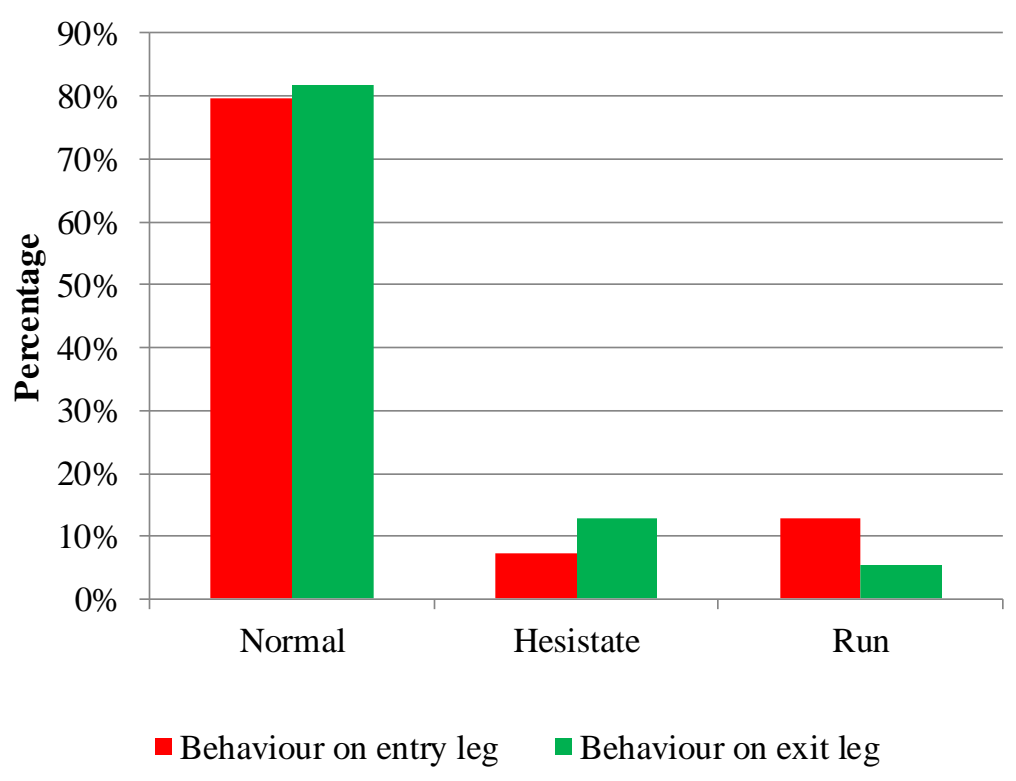

Figure 4.39 Crossing behaviour of pedestrians when a vehicle is present.

\subsubsection{Pedestrian Violations}

In approach $\mathrm{C}$, pedestrian-vehicle conflicts occurred before the crosswalk at the exit lane. As shown in Figure 4.40, pedestrians in approach $\mathrm{C}$ have spatial violation when crossing. It has been found that landscaping at the side of the entry leg helps to direct pedestrians to the proper crossing space. This landscaping is not available in the exit leg side, and it most probably leads to the pedestrian spatial violations when crossing, with some violations turned to generate conflicts with exiting vehicles. 

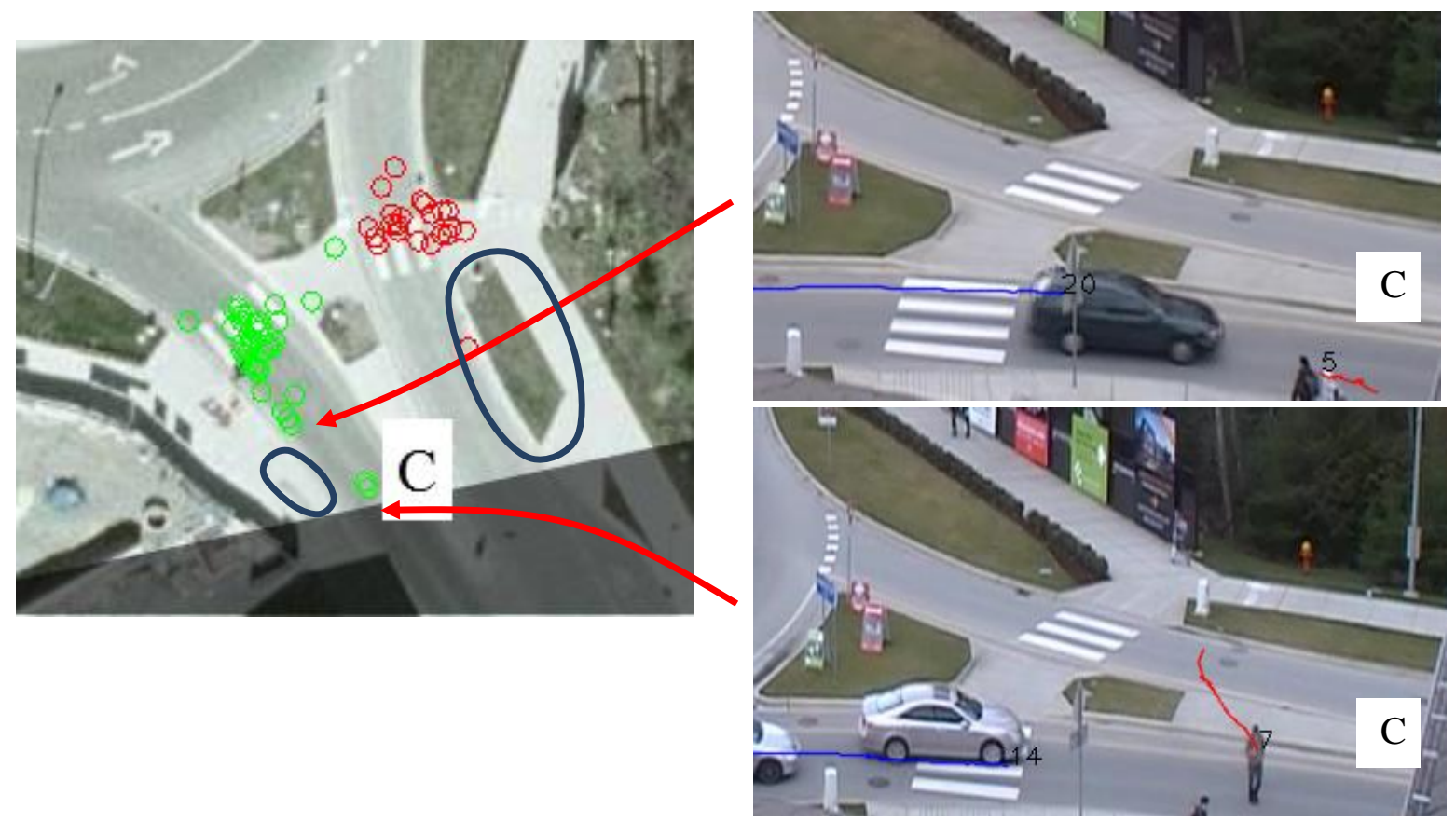

Figure 4.40 Pedestrian spatial violations in crossing in the exit lane on approach $\mathrm{C}$.

\subsection{Vehicle-cyclist Conflicts}

A vehicle-cyclist conflict is defined as an interaction between a vehicle and a cyclist which requires that one or both have to react or stop suddenly to avoid a collision. To better understand the safety of cyclists in the roundabout, it is important to classify cyclists' behaviour as follows:

- Cyclists behave as vehicles: by riding though entering, exiting, and circulating lanes

- Cyclists behave as pedestrians: by leaving vehicle lanes and riding though pedestrian crossings, or getting off the bike and continue walking.

At this particular roundabout, a road marking was placed on all approaches before the roundabout to direct cyclists to use the pedestrian sidewalks as shown Figure 4.41. However, some cyclists have used the vehicles lane and circulate with other vehicles. Therefore, this 
roundabout can be considered as a mix traffic roundabout that accommodates cyclists with vehicles lanes.
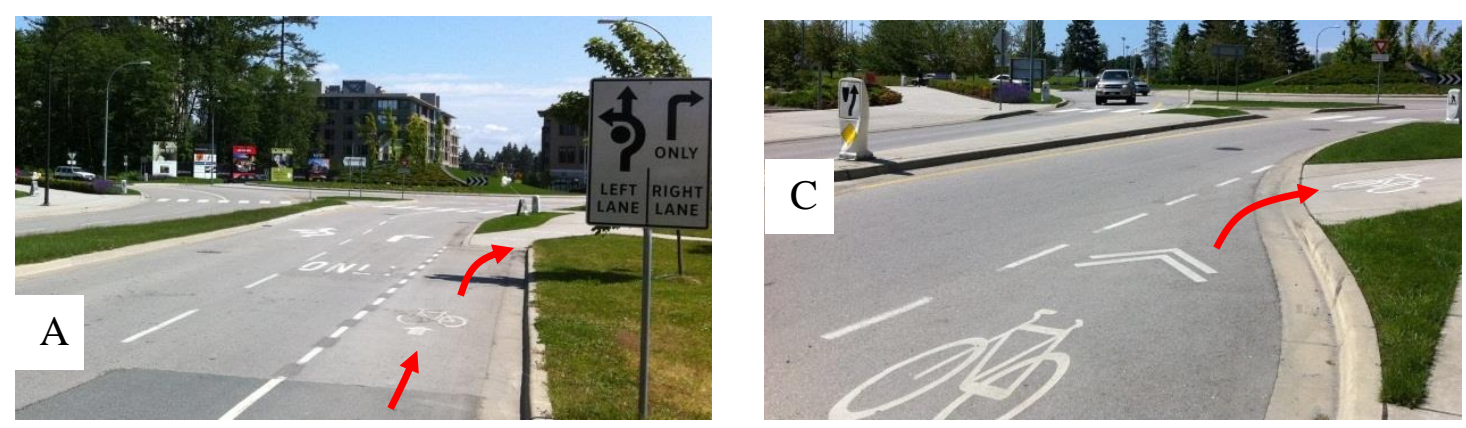

Figure 4.41 The road marking placed before the roundabout to direct cyclists.

There are approximately 69 vehicle-cyclist conflicts in the two-day analysis. These conflicts have recorded a TTC value of less than 1.5 seconds (45 frames). As shown in Figure 4.42, 47 cyclists behaved as vehicles, and 22 cyclists behaved as pedestrians. The roundabout design is a mix between two cyclist facility designs; the mixed traffic, and the separate cycle path. This can be implied from the dashed line separating the cycle approach lane with the vehicle approach lane as shown in Figure 4.41.

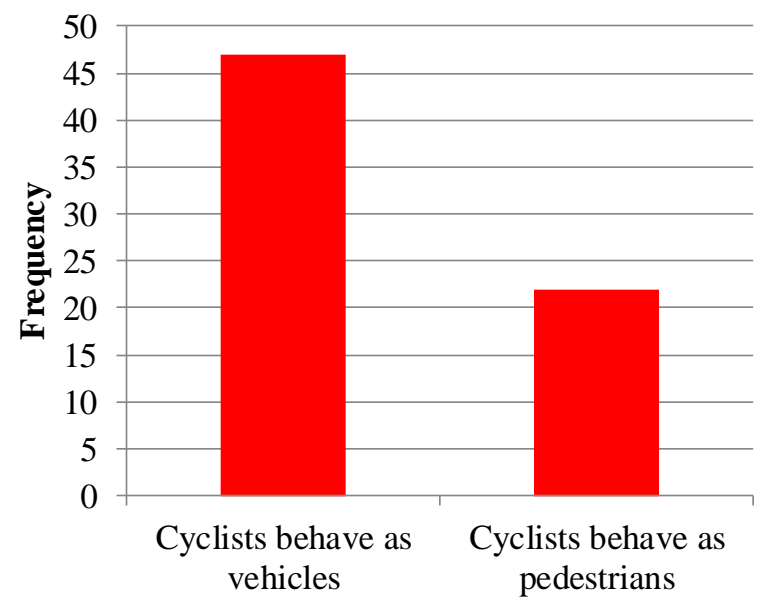

Cyclist behaviour

Figure 4.42 Frequency of cyclist behaviour types. 


\subsubsection{Conflicts of Cyclists Behaving as Vehicles}

There were approximately 47 vehicle-cyclist conflicts where the cyclist behaved as a vehicle. The classification of vehicle-cyclist conflicts follows the classification of vehicle-vehicle conflicts. Figure 4.43 shows the frequency of vehicle-cyclist conflict types.

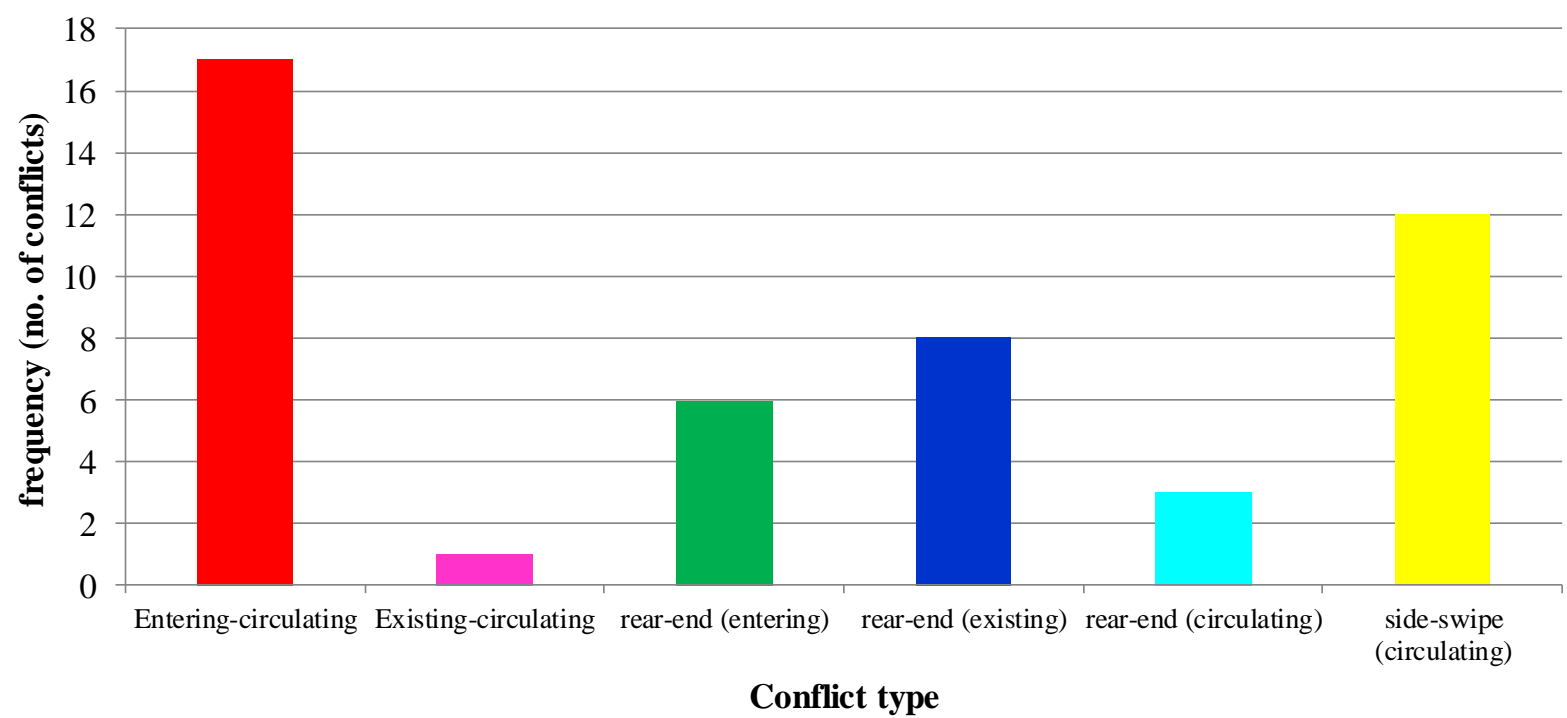

Figure 4.43 Frequency of vehicle-cyclist conflicts types

Figure 4.44 shows the points of vehicle-cyclist conflicts. Generally, most of the vehicle-cyclist conflicts occurred between approaches $\mathrm{C}$ and $\mathrm{B}$. 


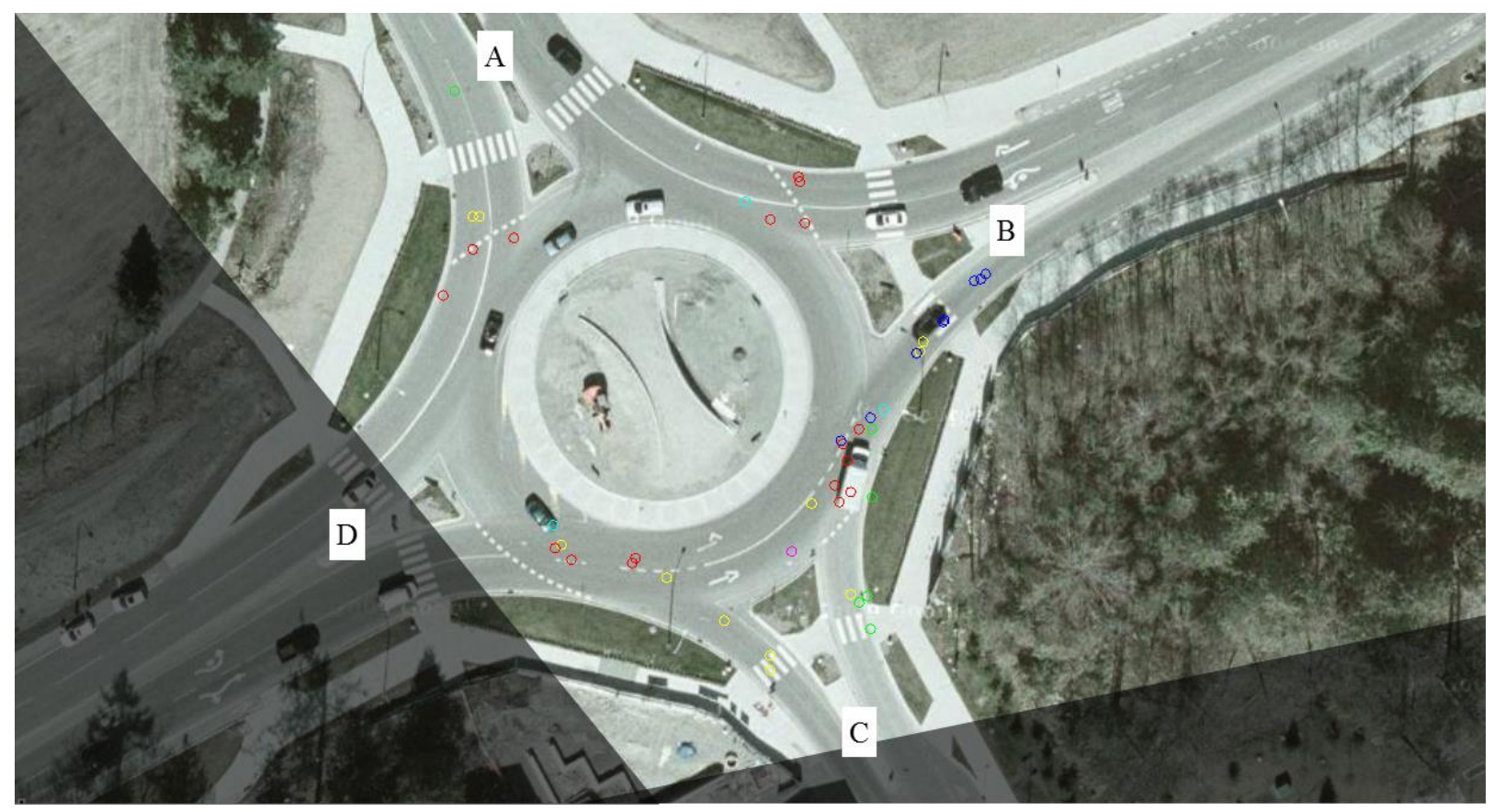

Figure 4.44 Vehicle-cyclist conflicts when cyclists behave as vehicles.

By reviewing vehicle-cyclist conflicts, behaviour of cyclists, and conflict causes are interpreted as follows:

- There are roughly 17 entering-circulating vehicle-cyclist conflicts in the roundabout, and Figure 4.45 shows examples of these conflicts. Due to their infrequency, it is difficult to determine a pattern or a cause of these conflicts. However, it was found that vehicles were generally confused when a cyclists was present, but usually tried to accommodate them. 

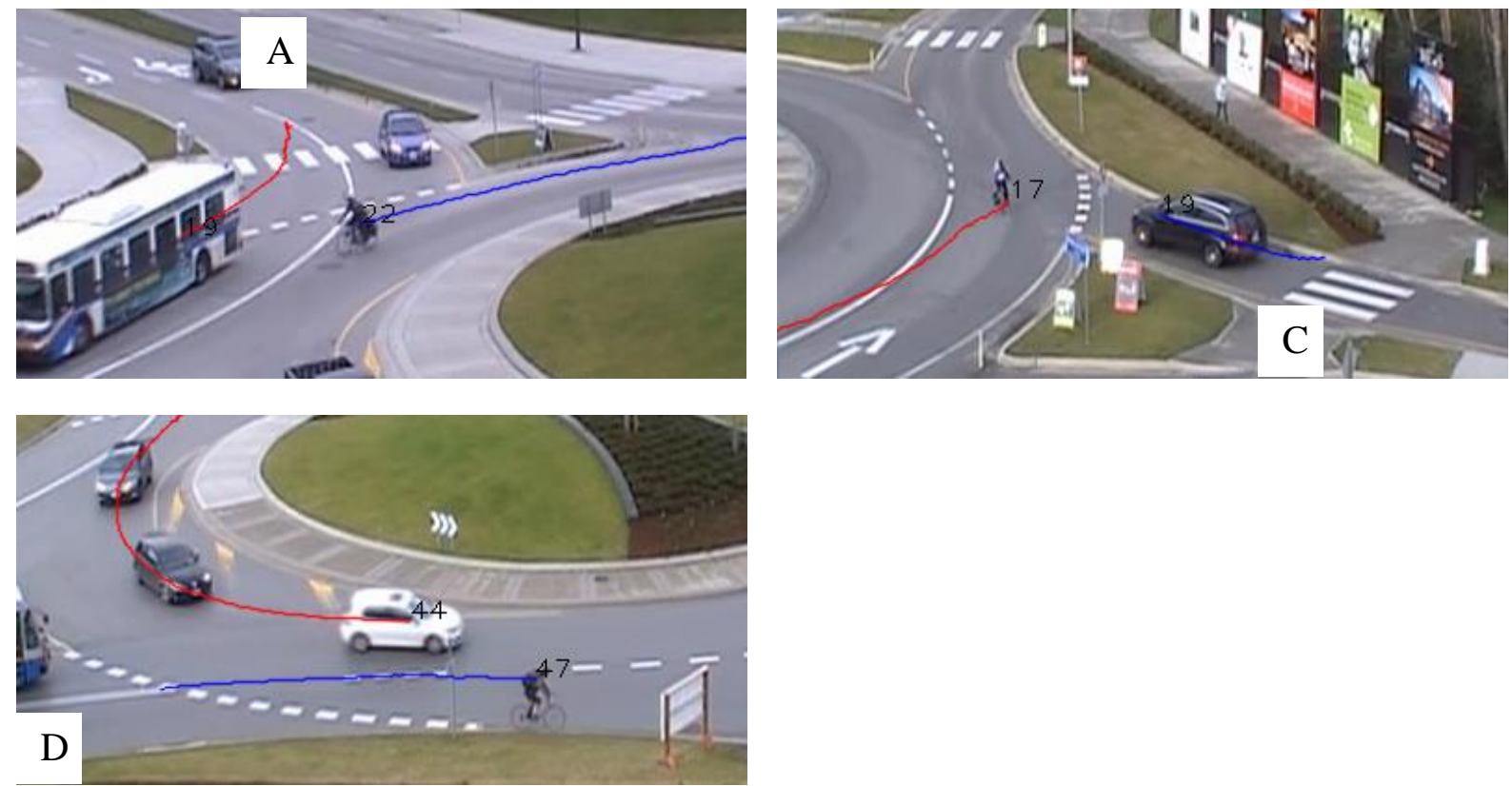

Figure 4.45 Examples of entering-circulating vehicle-cyclist conflicts.

- Entering-circulating conflicts can be analyzed based on yielding behaviour (Sakshaug, Laureshyn, Svensson, \& Hyden, 2010). Table 4.7 shows the yielding behaviour for vehicle-cyclist entering-circulating conflicts. There were 9 out of 17 entering-circulating conflicts where vehicles were entering the roundabout and cyclists were circulating. In five of the nine conflicts, motorists did not yield for cyclists and kept their movement unchanged. In eight out of the 17 entering-circulating conflicts, vehicles were circulating the roundabout and cyclists were entering from approaches. In two out of the eight conflicts, cyclists did not yield for vehicles and they either adjusted their behaviour or kept it unchanged. Since the numbers in these conflicts are relatively small, further investigation is required. 
Table 4.7 Yielding behaviour of motorists and cyclists in entering-circulating conflicts

\begin{tabular}{|c|c|c|c|c|c|c|}
\hline $\begin{array}{c}\text { Interaction } \\
\text { type }\end{array}$ & $\begin{array}{c}\text { Who should } \\
\text { yield? }\end{array}$ & $\begin{array}{c}\text { Who } \\
\text { yields? }\end{array}$ & \multicolumn{4}{|c|}{ Behaviour } \\
\hline & & & $\begin{array}{l}\text { cyclist } \\
\text { adjusts }\end{array}$ & $\begin{array}{c}\text { cyclists } \\
\text { unchanged }\end{array}$ & $\begin{array}{l}\text { motorist } \\
\text { adjusts }\end{array}$ & $\begin{array}{c}\text { motorists } \\
\text { unchanged }\end{array}$ \\
\hline Entering-circ & & Motorists & 4 & 4 & & \\
\hline (mot-entering) & inturists & Cyclists & 5 & & & 5 \\
\hline Entering-circ & 1. & Motorists & 2 & 1 & & \\
\hline (cyc-entering) & & Cyclists & 6 & & 1 & 5 \\
\hline
\end{tabular}

- Figure 4.46 shows examples of rear-end (entering) vehicle-cyclist conflicts. Out of six conflicts, five conflicts involved vehicles following a cyclist. In these five conflicts, vehicles were usually aware of the cyclist, and they appeared to accommodate the cyclist even though they were riding on the edge of the lane.
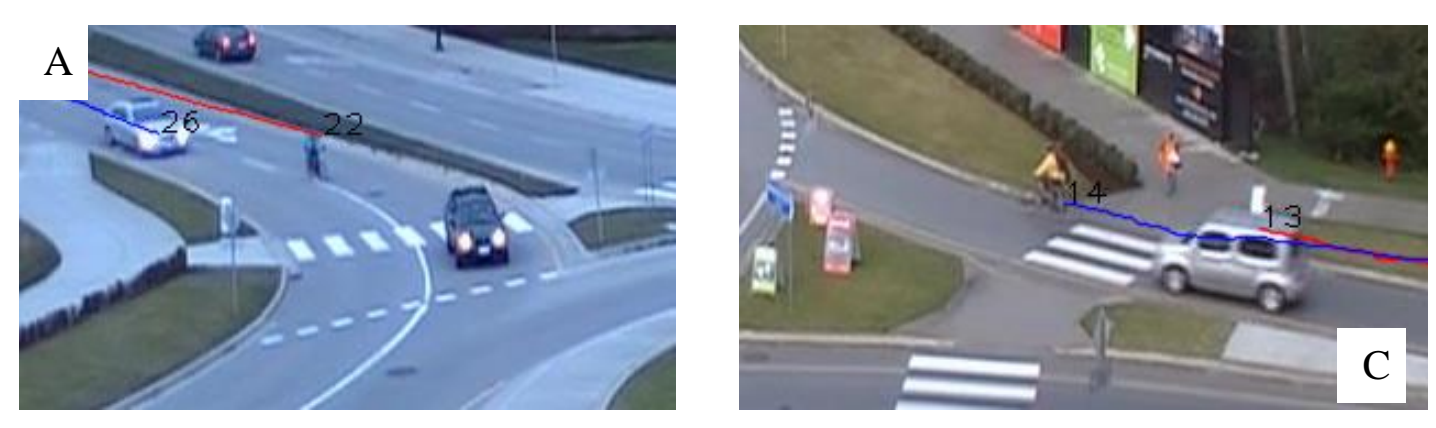

Figure 4.46 Examples of rear-end (entering) vehicle-cyclist conflicts.

- Similar behaviour has been noticed in rear-end conflicts in exiting lanes. Figure 4.47 shows examples of rear-end (exiting) vehicle-cyclist conflicts. In seven of eight conflicts, vehicles followed cyclists but tried to be accommodating. 

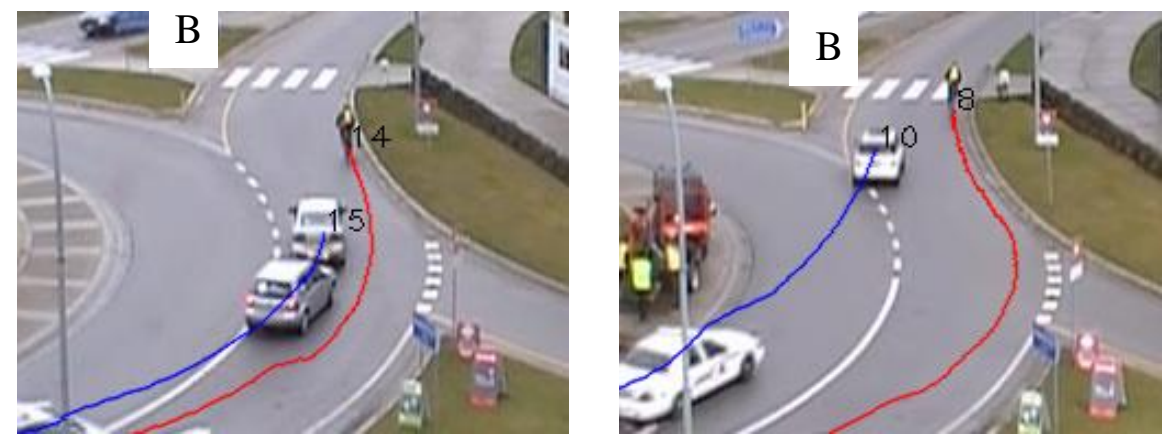

Figure 4.47 Examples of rear-end (exiting) vehicle-cyclist conflicts.

- In rear-end conflicts occurring in circulating lanes, two of three conflicts occurred when cyclists were following vehicles. Figure 4.48 shows examples of rear-end vehicle-cyclist conflicts occurring in circulating lanes.
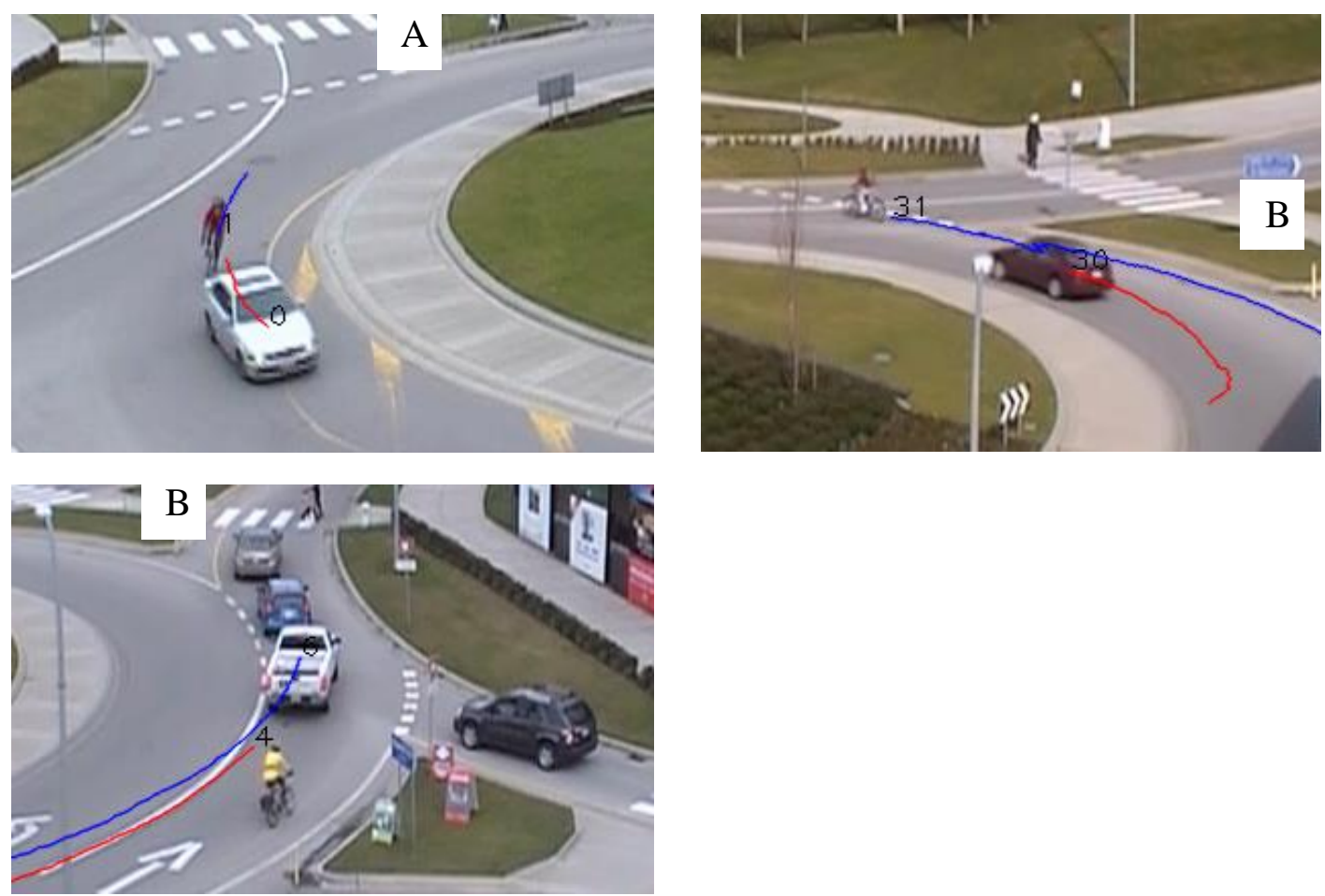

Figure 4.48 Examples of rear-end (circulating) vehicle-cyclist conflicts. 
- There are 12 sideswipe conflicts in the roundabout, with examples shown in Figure 4.49.
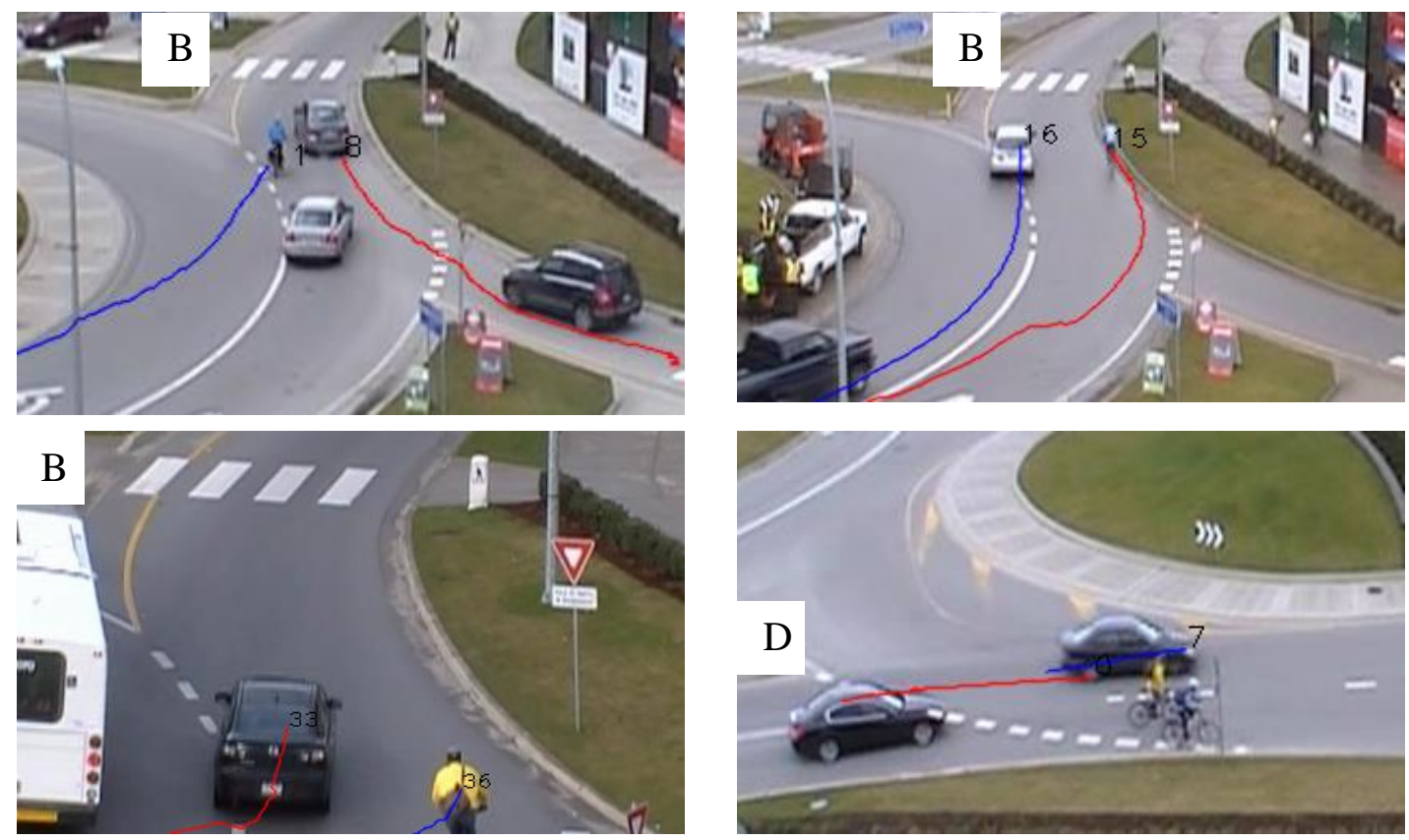

Figure 4.49 Examples of sideswipe vehicle-cyclist conflicts.

\subsubsection{Conflicts of Cyclists Behaving as Pedestrians}

There were roughly 22 vehicle-cyclist conflicts when cyclists behaved as pedestrians, with 15 of 22 conflicts occurred at approach C. By reviewing all conflicts, motorist-cyclist behaviour and conflict causes are interpreted as follows:

In approach $\mathrm{C}$, five of the 15 conflicts occurred when a cyclist crossed from the island to either the exit or the entry lane. It was found that some cyclists who crossed from the island are less cautious about conflicting with vehicles, and either seems to be unaware of the conflicting 
vehicle

or

assumed

the

vehicle

would

stop.

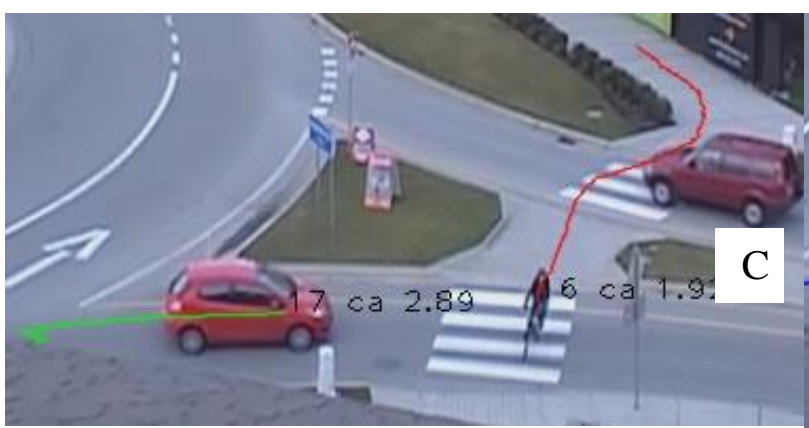

(a)

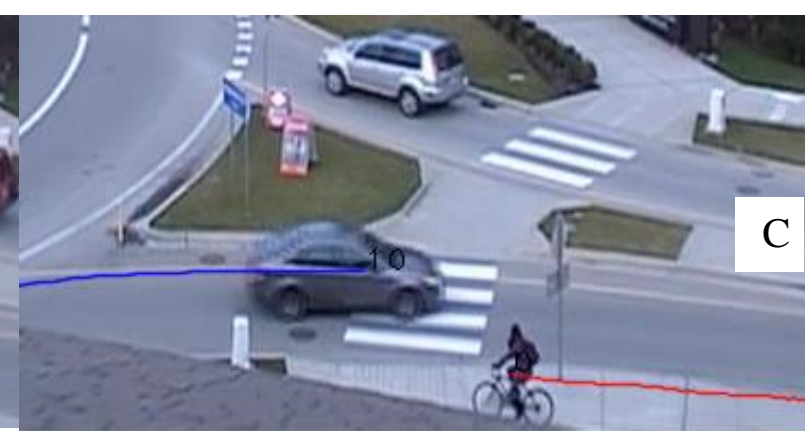

(b)

- Figure 4.50 provides two examples of vehicle-cyclist a conflict behave as a pedestrian.

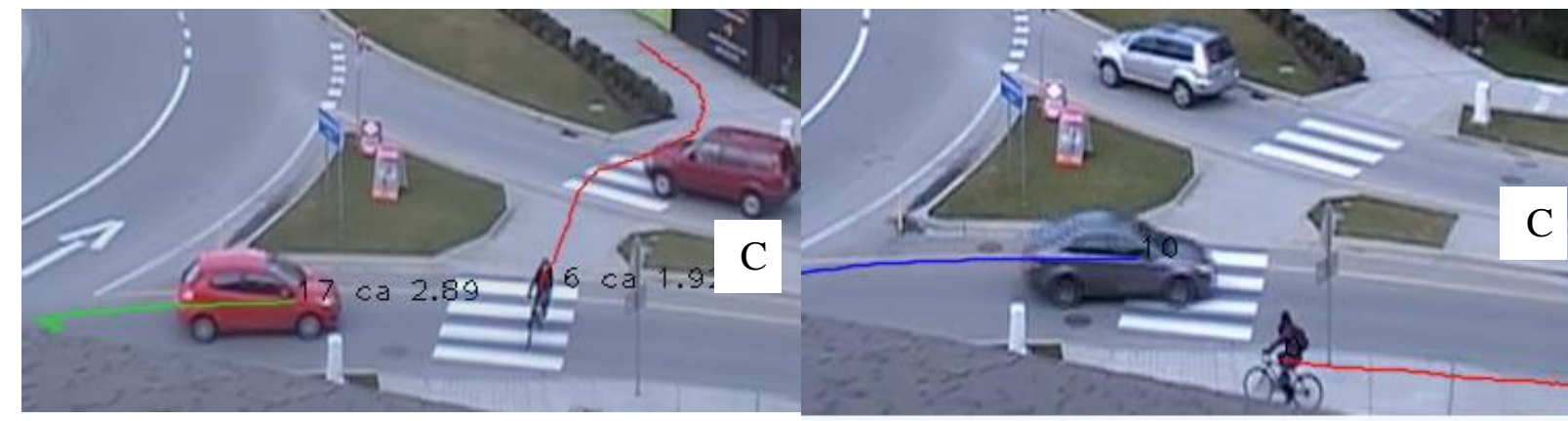

(a)

(b)

Figure 4.50 Vehicle-cyclist conflicts when (a) a cyclist is crossing from island, and (b) a cyclist is crossing from side.

\subsection{Cyclist-cyclist Conflicts}

There were five cyclist-cyclist conflicts in the roundabout, where three of the five conflicts occurred between cyclists that behaved as vehicles. There were two rear-end conflicts and one sideswipe conflict. The remaining two conflicts occurred when at least one cyclist behaved as a pedestrian. Figure 4.51 shows examples of cyclist-cyclist conflicts. 

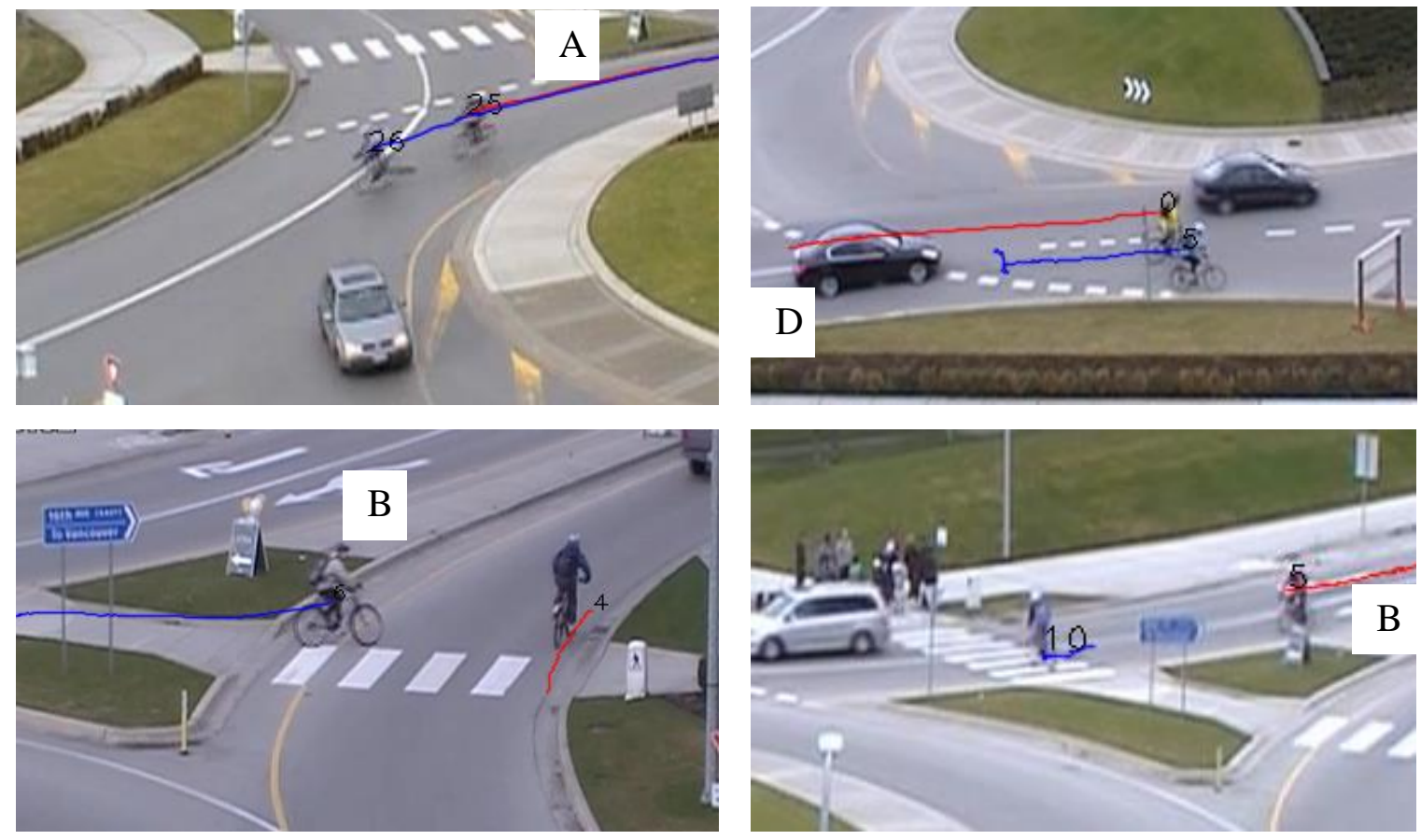

Figure 4.51 Examples of cyclist-cyclist conflicts.

\subsection{Cyclist-pedestrian Conflicts}

There were 10 cyclist-pedestrian conflicts in the roundabout, where six of the ten conflicts occurred when cyclists behaved as pedestrians. Figure 4.52 shows examples of cyclist-pedestrian conflicts. This raises the concern about accommodating cyclists in pedestrian facilities. Cyclists travelling at high speeds affect how pedestrians perceive their safety and comfort. On the other hand, if cyclists are not accommodated in the mixed traffic roundabout, cyclist will have to take off the bike and walk through as pedestrians. This also affects how cyclists perceive their mobility and comfort in roundabouts. 

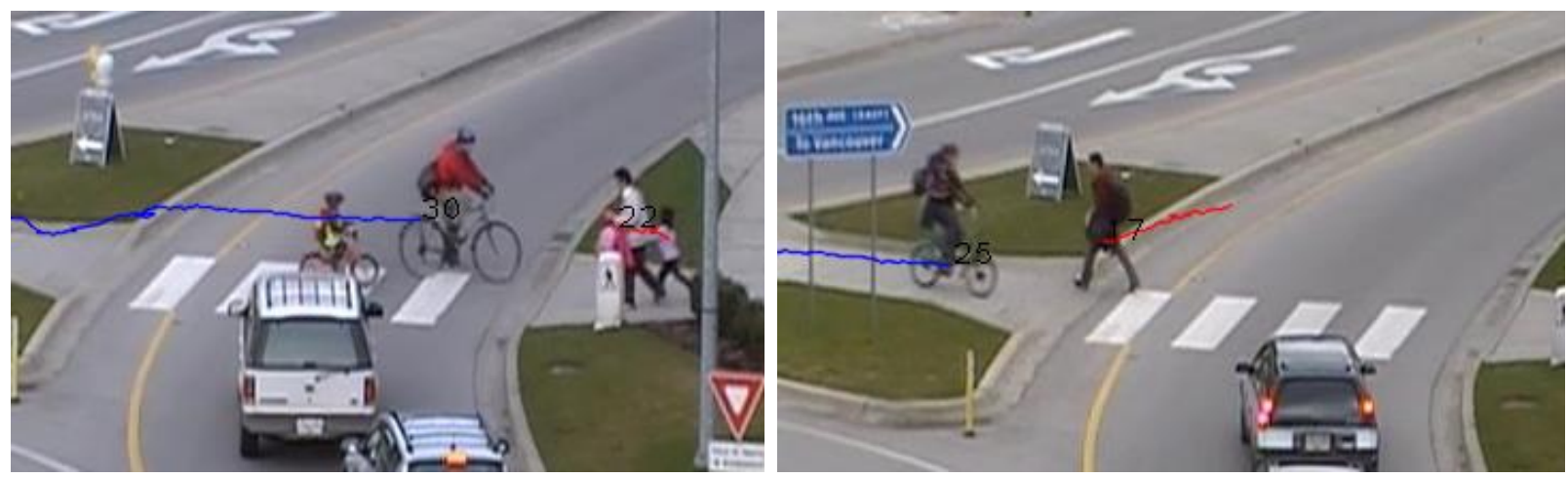

Figure 4.52 Examples of cyclist-pedestrian conflicts.

\subsection{Overall Assessment of All Conflicts:}

Across all roundabout conflicts, there were some commonalities and key differences between conflicts types. Some of the vehicle-vehicle conflicts occurred because of the interaction with the pedestrian crossing. This resulted in the generation of vehicle-pedestrian conflicts at crosswalks. Vehicle-cyclists conflicts were similar in nature of vehicle-vehicle conflicts when cyclists behaved as vehicles. Vehicle-cyclist conflicts were also similar in nature of vehicle-pedestrian conflicts when cyclists behaved as pedestrians.

Cyclist-cyclist conflicts and cyclist-pedestrian conflicts were similar in the nature of their behavior. Both cyclists and pedestrian contributed to a conflict were usually aware and accommodating the other road user at the interaction. This explains the low severity of these conflicts.

It is important to note that all vehicle-vehicle and vehicle-pedestrian conflicts types are generic types that can occur in any other roundabout. However, vehicle-cyclist, cyclist-cyclist, and cyclist-pedestrian conflicts are specific to roundabout that is designed as a mixed traffic roundabout that accommodates cyclists with vehicles in the same lane. 


\subsection{Conflicts and Accidents Contributory Factors}

Montella (2007) identified some limits for the roundabout design elements that affect the safety of the roundabout and are considered as accident contributory factors. These limits are used in this section to check the design of the roundabout. This will help in linking the unsatisfied design elements limits with the conflicts generated at the roundabout.

\subsubsection{Geometric Design}

Some of the geometric design elements of the roundabout can be a contributing factor for some of the conflicts. Montella (2007) identified several geometric design factors as collision contributory factors. The group of geometric design factors was associated with a collision type, if at least one geometric design factor is linked to that specific collision type. It is hard to determine the exact geometric design factor that is associated with a collision type. However, the following sections discuss the most relevant geometric factors that may affect the safety of the roundabout.

\subsubsection{Exit lane radius}

The maximum threshold value for the identification of high risk issue for the exit lane radius is 50 meters (Montella A. , 2007). As shown in Figure 4.53, the radius of the exit lane on approach B is approximately 49 meters (which is very close to the threshold), and this may be associated with the frequent exiting-circulating conflicts at approach B. The large exit radius was a major contributory factor in $10.6 \%$ of the collisions in 15 roundabouts in Italy (Montella A. , 2011). 


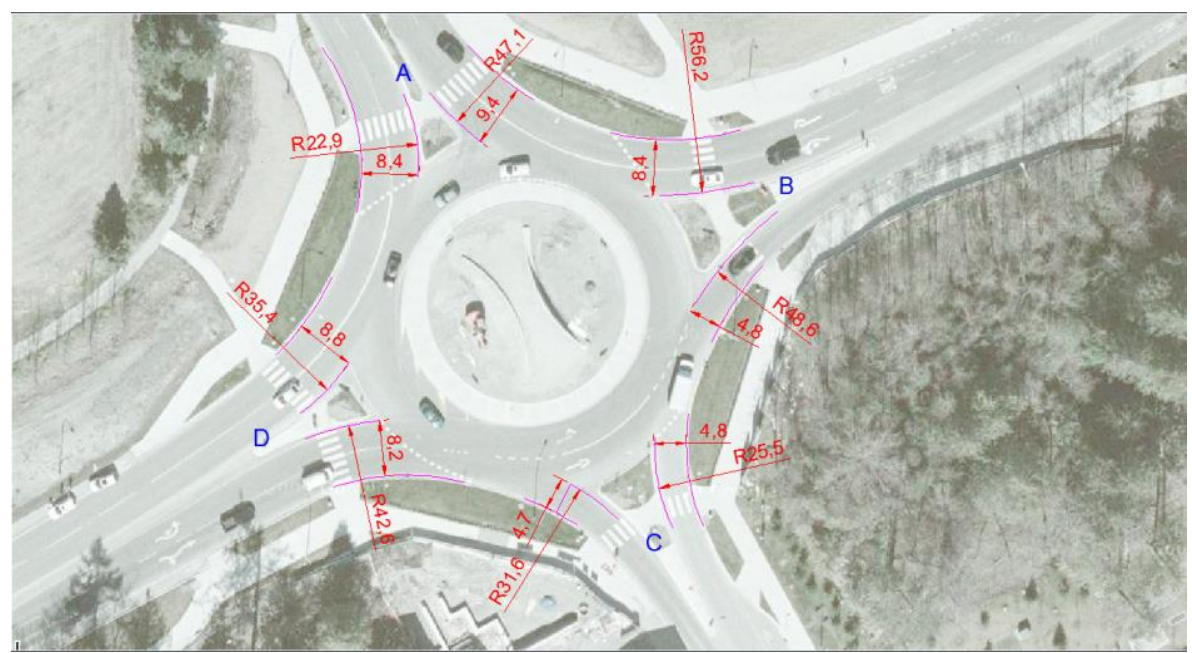

Figure 4.53 Radiuses and widths of exit and entry lanes.

\subsubsection{Entry angle}

The minimum threshold value for the identification of high risk issue for the entry angle is 30 degrees (Montella A. , 2007). As shown in Figure 4.54, all entry angles for all approaches are larger than 30 degrees.

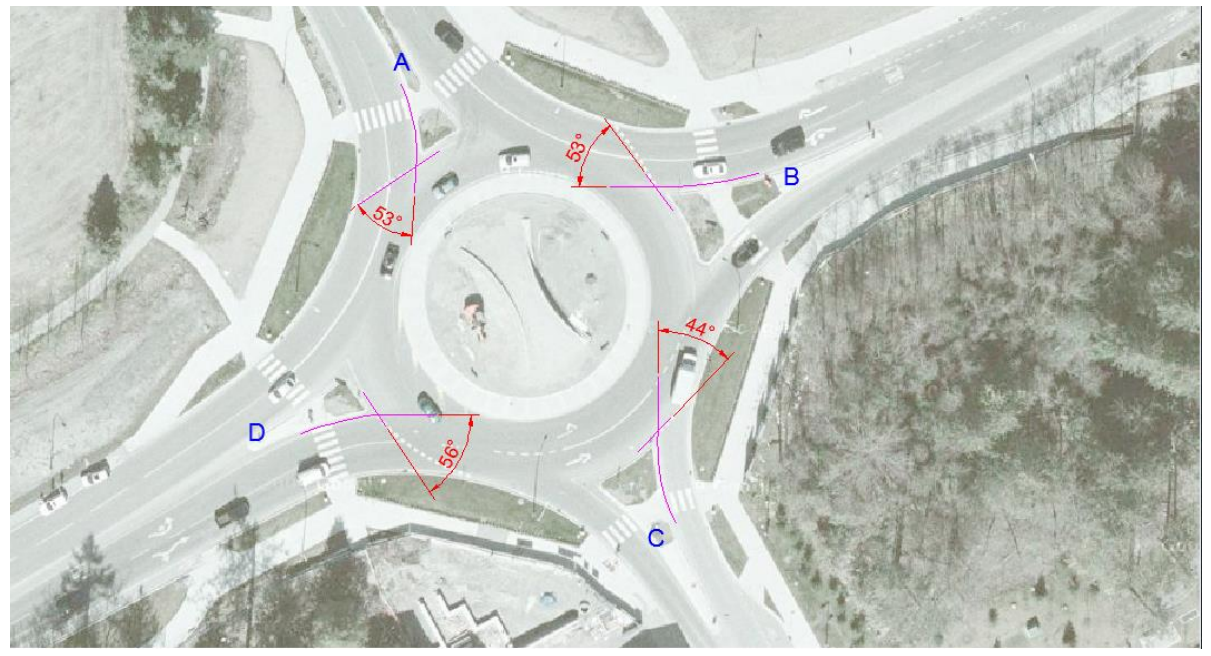

Figure 4.54 Entry angles in all approaches. 


\subsubsection{Radius of deflection}

The maximum threshold value for the identification of high risk issue for the radius of deflection is 100 meters (Montella A. , 2007). As shown in Figure 4.55, the radius of deflection for all approaches is much less than 100 meters.

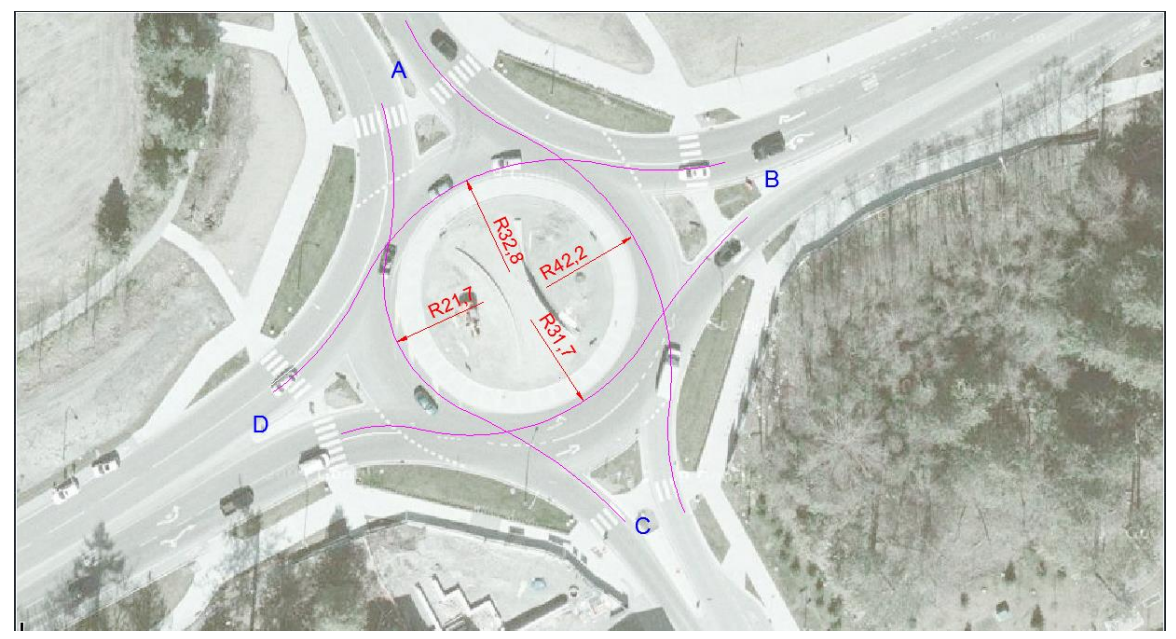

Figure 4.55 Radius of deflection for all entering approaches

\subsubsection{Deflection (deviation) Angle}

The minimum threshold value for the identification of high risk issue for the deflection (or called deviation) angle is 30 degrees (Montella A. , 2007). As shown in Figure 4.56, all deflection angles for all approaches are larger than 30 degrees. 


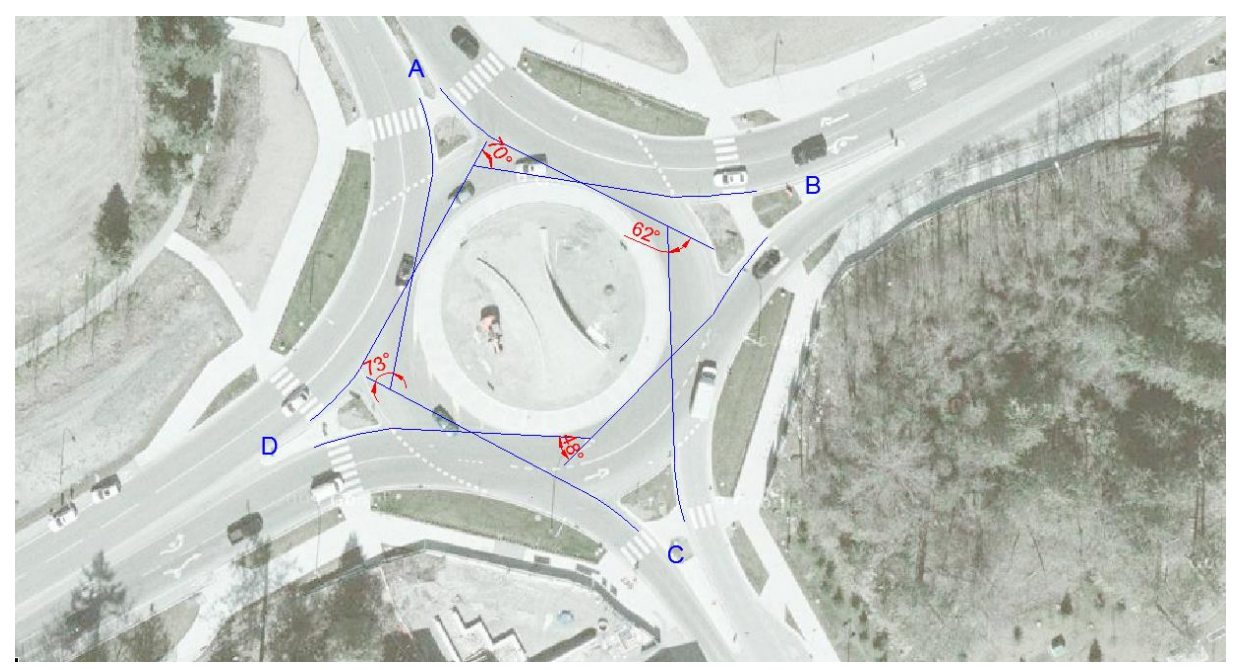

Figure 4.56 Deflection angles for all entering approaches.

\subsubsection{Intersection sight distance}

The Intersection sight distance is also important to validate the roundabout geometric design ( National Cooperative Highway Research Program (NCHRP), 2010). The design speed of entering vehicles is $50 \mathrm{~km} / \mathrm{hr}$ and by assuming a value of 3.6 seconds for reaction and braking time, the distance required to stop for both entering and circulating vehicles can calculated.

Entering velocity $=50 \mathrm{~km} / \mathrm{hr}=13.89 \mathrm{~m} / \mathrm{sec}$

Distance required $=\mathrm{V} * \mathrm{t}_{\mathrm{R}}=13.89 \mathrm{~m} / \mathrm{sec} * 3.6 \mathrm{sec}=50 \mathrm{~m}$

Figure 4.57 and Figure 4.58 show the intersection sight distance check for approaches A, B, C, and D. The sight distances required between entering and circulating vehicles for all approaches are adequate. 


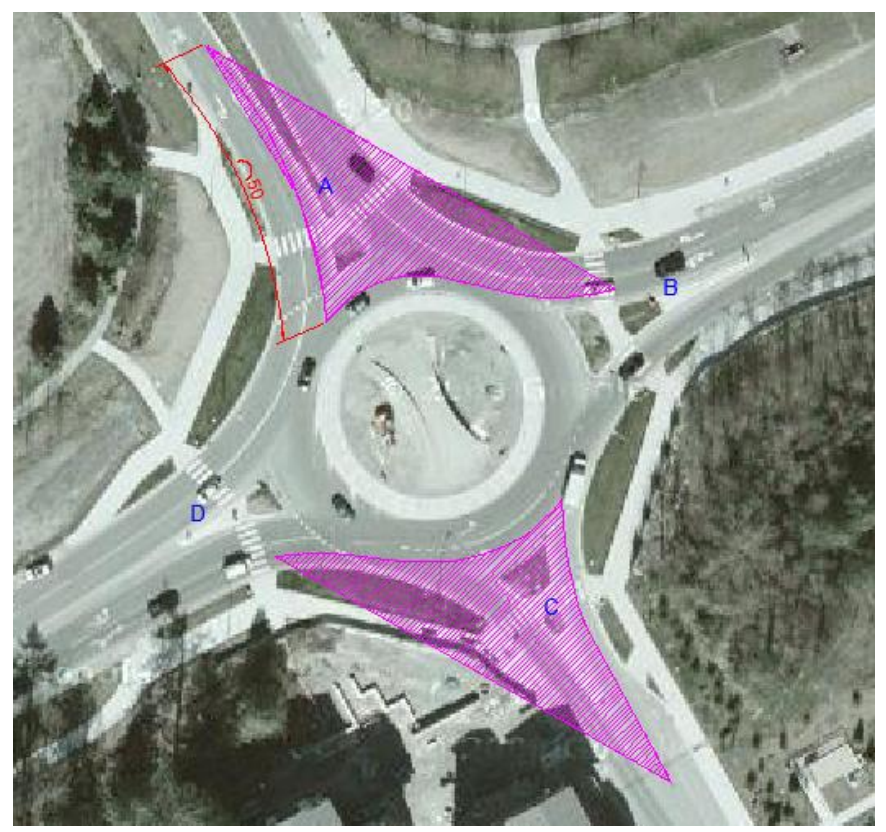

Figure 4.57 Sight distance check for approaches A and C.

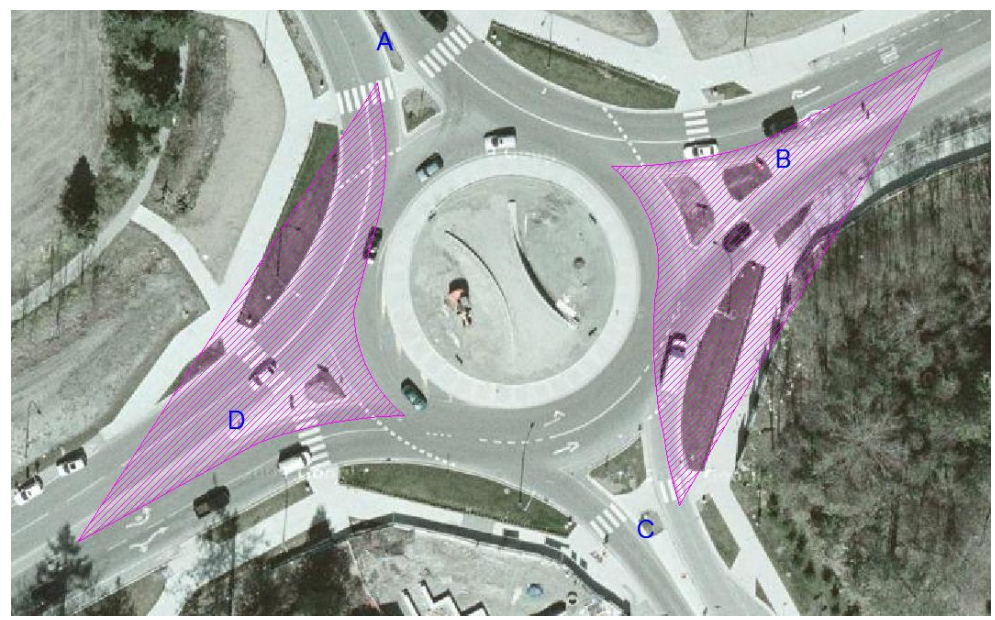

Figure 4.58 Sight distance check for approaches B and D.

\subsubsection{Road Marking}

Road markings were considered a contributory factor in approximately $51.8 \%$ of collisions in the collision contributory factors identification research (Montella A. , 2011). It was found that missing or faded lane separation in roundabouts was considered a major factor in $4.7 \%$ of 
collisions. In this roundabout, marking of the central island is faded which seems to confuse drivers circulating the roundabout in front of approach D. The confusion of entering vehicles from approach D may have resulted because of a wrong perception of a circulating vehicle position near the faded marking. Figure 4.59 shows several locations of faded or poorly designed marking at the roundabout. The confusion between vehicles circulating vehicles and exiting vehicles on approach $\mathrm{C}$ may be due to poorly designed marking. Similar confusion between circulating and exiting vehicles on approach B may be due to the faded marking.

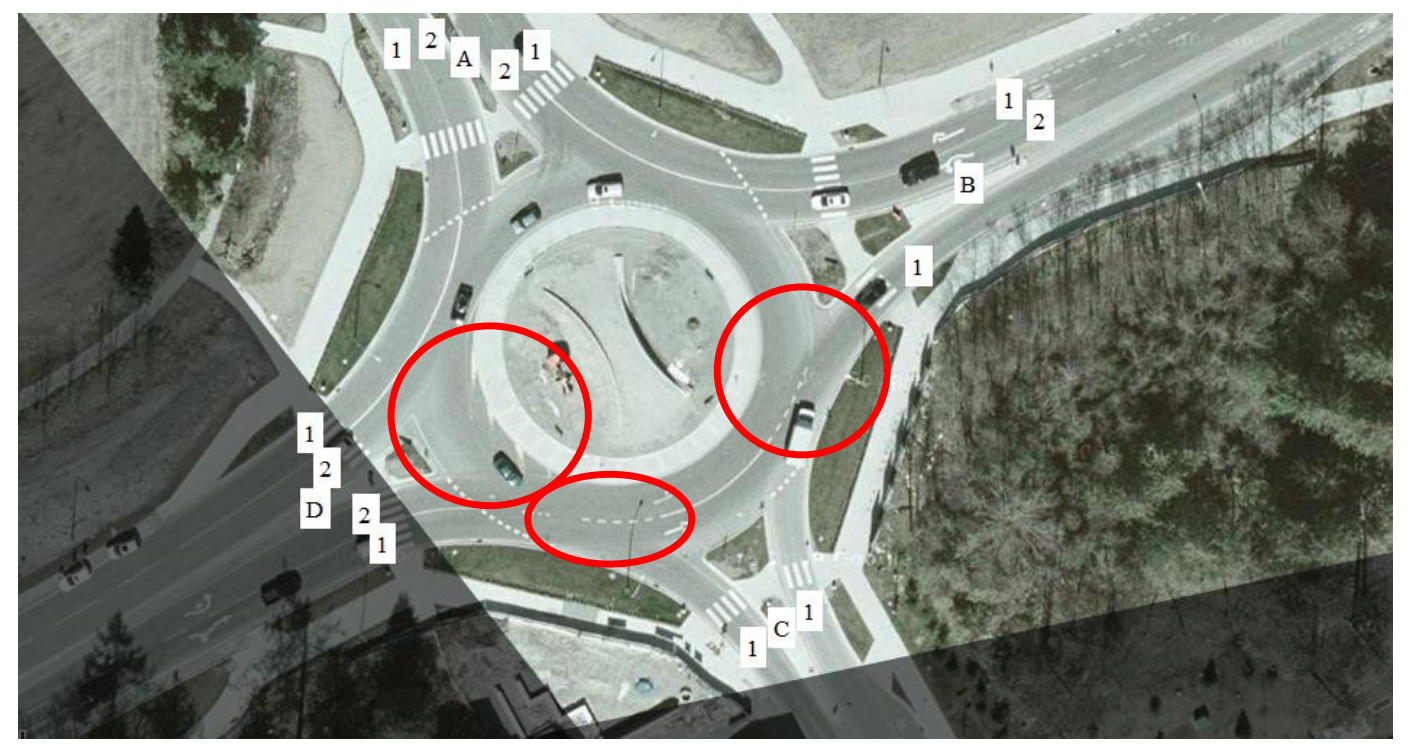

Figure 4.59 Faded or poorly designed road markings.

\subsubsection{Signs}

Signs were considered a contributory factor in roughly $39.1 \%$ of collisions in the collision contributory factors identification research (Montella A. , 2011). It was found that missing, faded, or poorly located yield signs at roundabouts were considered a major factor in $18.2 \%$ of collisions. In this roundabout, the yield signs are poorly located and ineffective at all approaches as was shown in Figure 4.14. 


\section{Recommendations}

A diagnostic safety analysis has been performed for the purpose of demonstrating safety concerns and developing an in-depth understanding of the behaviour of road users at the roundabout. This chapter presents several recommendations that are proposed based on the completed analysis.

\subsection{Road Marking}

Driver confusion at the roundabout may be reduced if road markings are modified. Based on the completed safety analysis, several road marking modification are proposed as shown in Figure 5.1.

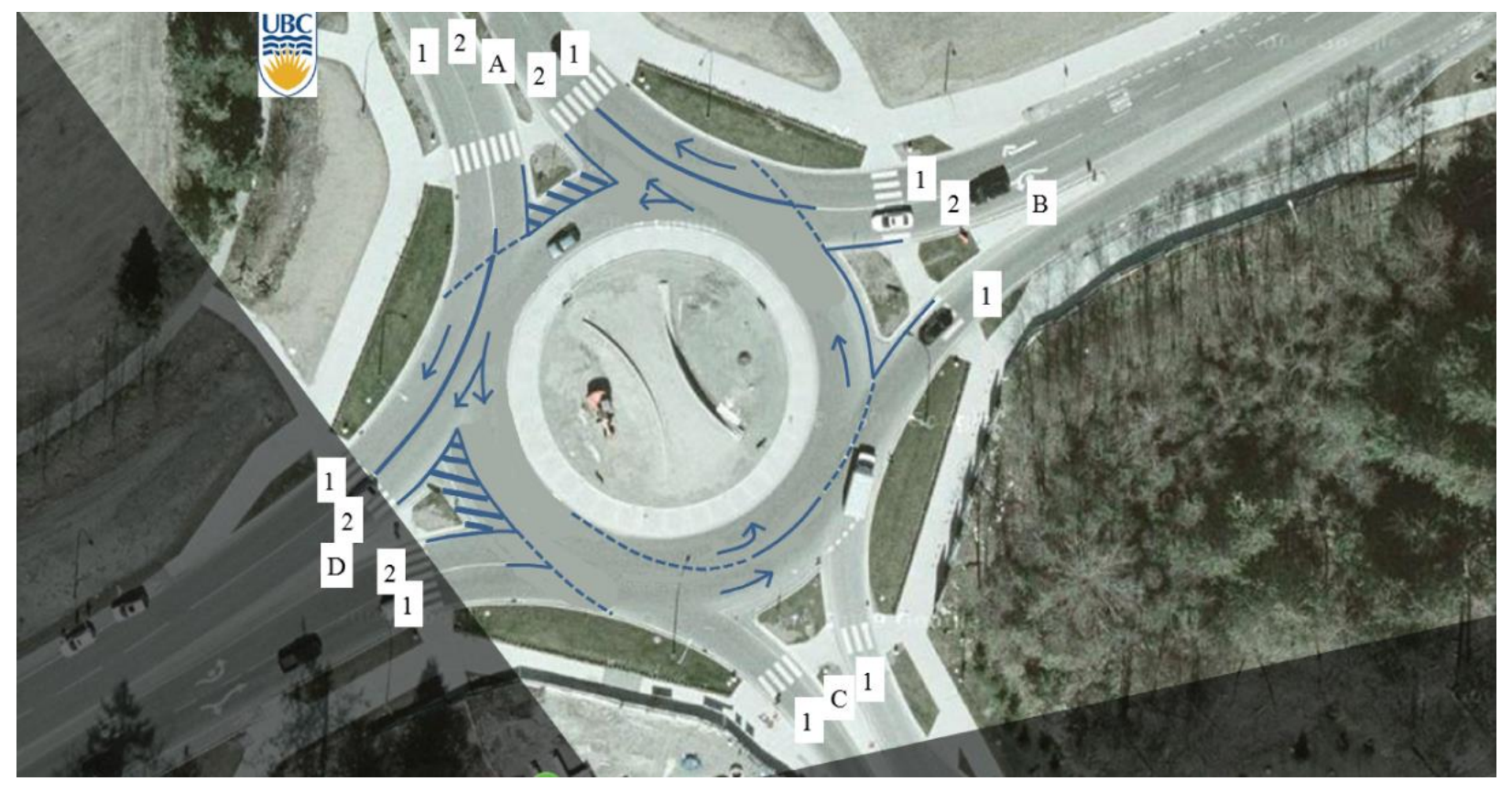

Figure 5.1 Proposed road markings modifications.

The circulating lanes in front of approaches $\mathrm{A}$ and $\mathrm{D}$ are suggested to be narrowed down to help directing vehicles to a one-vehicle occupancy lane. Cross hatchings are suggested to be added to 
the splitter island marking, as that will help that entering vehicles will clearly perceive the rightof-way of circulating vehicles. This will ensure entering vehicles yield for circulating vehicles without being confusion over a possible wrong negotiation. This was noticed in the manual review of conflicts occurring between entering and circulating vehicles at approaches A and D. This recommendation cannot be applied to approach $\mathrm{B}$, because the circulating lane is already narrowed down, and cannot be applied also to approach $\mathrm{C}$, because the circulating lanes are already narrowed down to two lanes.

It is suggested that the separation lane lines for the protected right turn lanes between approaches $\mathrm{B}$ and $\mathrm{A}$, and between approaches $\mathrm{A}$ and $\mathrm{D}$ should be wide to assure the right of way for right turning vehicles in the protected lane, and this is suggested because of the conflicts occurring between circulating and entering vehicles at this area. After reviewing these conflicts, it was found that circulating vehicles were confused when interacted with entering vehicles occupying the protected right turn. Widening the separation line would help circulating vehicles to correctly perceive the protected right turn. The entering right lane should always have the yield line for circulating vehicles, even if there is a protected right turn lane. This may not mitigate conflicts found between circulating and entering vehicles, but it will help to recognize the expected maneuver of each vehicle.

It is recommended to eradicate the current spiral marking and dashed line in front of approach D. In addition, a new dashed line is suggested to be added to merge circulating vehicles (in front of approach D) to merge with approach $\mathrm{C}$ circulating lanes. This would help circulating vehicles to understand that by circulating the left lane, they will make them keep circulating without exiting to approach B. This marking modification should reduce frequent exiting-circulating conflicts when exiting on approaches $\mathrm{C}$ and $\mathrm{B}$. Some confusion may remain for some drivers, because one 
circulating lane is merged into two circulating lanes. In some cases, the design can be improved to allow an equal number of circulating lanes at the roundabout with equal number of entering and exiting lanes for all approaches.

It has also been suggested to enlarge the arrows markings in front of approach $\mathrm{C}$, and to place them closer to dashed line marking. In addition, an extra arrow marking should be placed at the beginning of the circulating lanes (in front of approach B and D), and two direction arrows be placed between approach B and A, and between approach A and D, to clarify proper negotiation.

\subsection{Signs}

Confusion of road users at the roundabout can be reduced if signs are properly modified. Based on the completed analysis, several sign modifications were proposed. Yield signs at the approaches should be enlarged to improve the yielding behaviour of entering vehicles. Furthermore, in accordance to the guidelines in MUTCD for traffic control devices (FHWA, 2009), large pedestrian signs should be placed at the splitter island, the entry and the exit lanes as shown in Figure 5.2. 


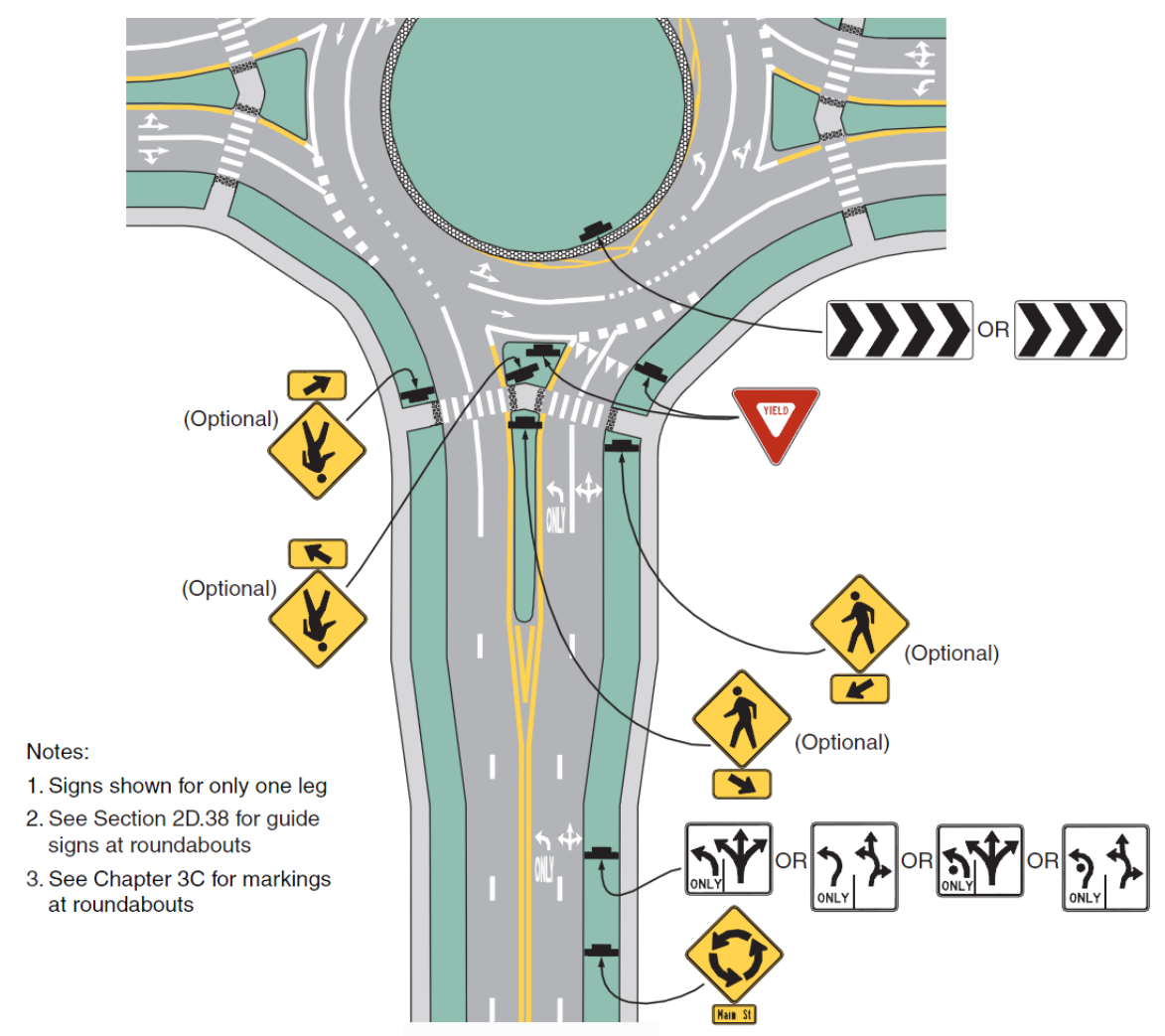

Figure 5.2 Suggested signs at multi-lane roundabouts as per the MUTCD (FHWA, 2009).

\subsection{Crosswalks}

Confusion of road users at the roundabout can be reduced if crosswalks' designs are modified.

Based on the completed analysis, several crosswalk design modifications are proposed. Furthermore, the crosswalk design can be improved by directing pedestrians to a visible crossing area. As found on approach $\mathrm{C}$ of the roundabout, the landscape area around at the entry leg side directed the pedestrians to the crosswalks and decreased jaywalking. Similar landscape areas can be placed on the exit leg side of approaches $\mathrm{C}$ and $\mathrm{A}$. The small landscape areas at the entry and exit leg sides on approach B should also be enlarged. Figure 5.3 presents the proposed landscape. 


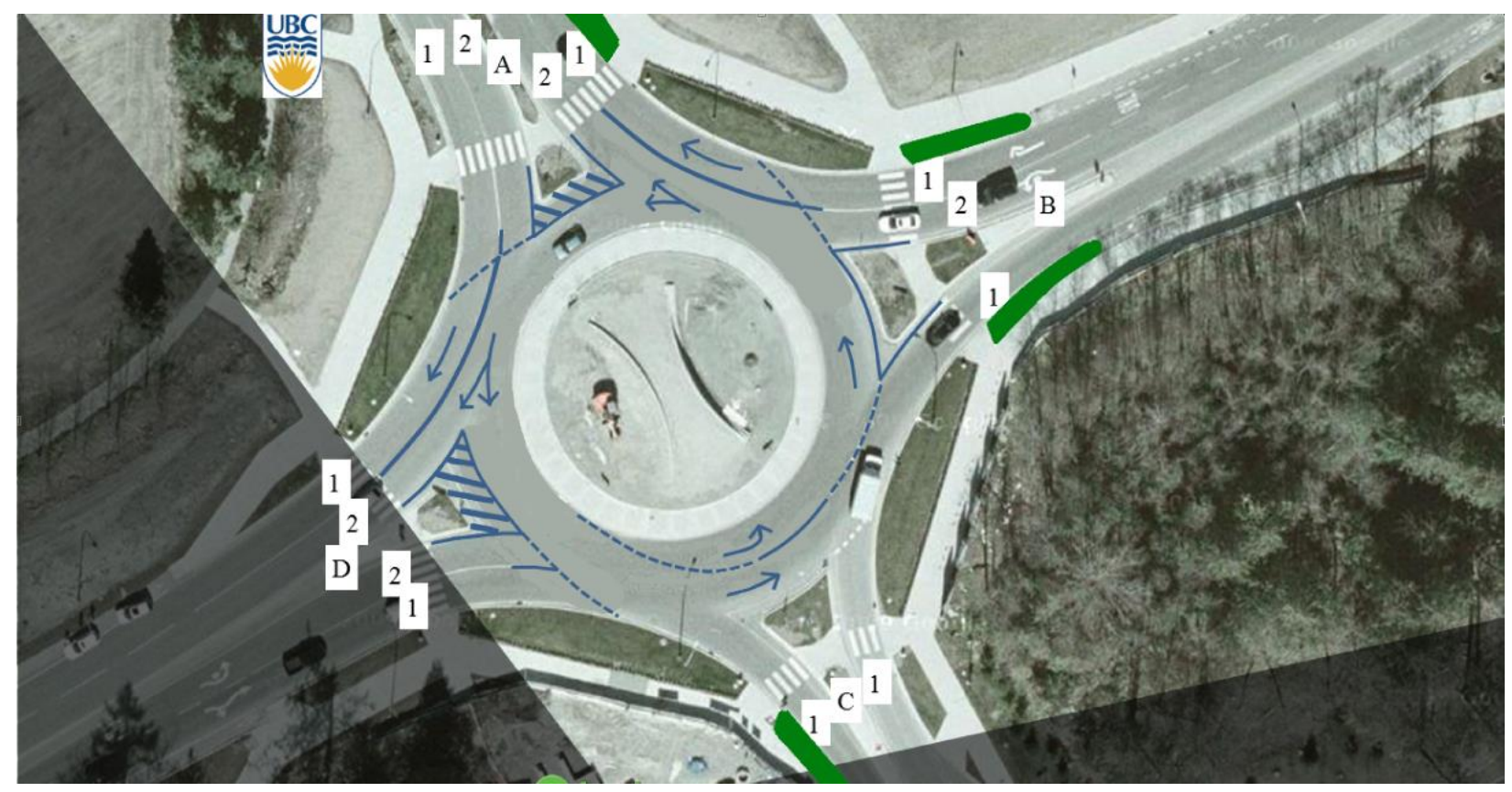

Figure 5.3 Suggested landscape on the approach sides

\subsection{Driver Education}

Safety at the roundabout can be improved if drivers, cyclists, and pedestrians are fully educated on their proper use. Roundabouts are relatively considered a new type of intersections in North America. More driver education is recommended as some motorists were unaware of the proper negotiations (as explained in the violations sections). The ICBC guide to driving in British (Learn to Drive Smart, 2011) includes just the main ruling that relate to roundabouts. As such, more detailed guides and instructions are required to inform drivers about the vehicle right-ofway and how to interact with entering and circulating vehicles. It is also significant to educate drivers on accommodating pedestrians, and especially at exit lanes. Although behaviour of drivers may be considered a long-term countermeasure, it will complement other engineering countermeasures to improve the overall safety of roundabouts. 


\section{Summary and Conclusions}

Safety at intersections is a recent concern for traffic safety specialists worldwide. There are more than 2.4 million road accidents annually at U.S. intersections (Insurance Institute for Highway Safety, 2008). Furthermore, roughly $29 \%$ and $43 \%$ of fatalities and serious injury collisions, respectively, occur at intersections throughout Canada (Road Safety Directorate, 2007). Based on these statistics, there has been a growing interest to replace regular intersections with roundabouts. Several studies ( (Schoon \& Minnen, 1993); (Rodegerdts, et al., 2007)) have shown that converting intersections to roundabouts improves the safety performance of that intersections. Therefore, as more roundabouts are being constructed throughout North America, a detailed study of their safety performance is increasingly required. Using collision data to evaluate the safety performance of roundabouts is considered a reactive and costly approach. The Traffic Conflict Technique (TCT) has recently been used to improve and complement the collision-based safety diagnosis techniques. The latest advanced automated video-based safety tool developed in UBC was recently used in several applications, such as before-and-after studies (Autey, Sayed, \& Zaki, 2011) and in the analysis of vehicle interactions (Saunier, Sayed, \& Ismail, 2010).

The purpose of this thesis was to demonstrate the use of the UBC automated safety tool in the diagnostic analysis for the safety at roundabouts. Traffic conflicts occurring at the roundabout were automatically identified and analyzed to develop an in-depth understanding of the behaviours of road users and the causes of traffic conflicts. Based on this fast and low-cost approach, effective countermeasures were proposed to proactively improve the safety of the roundabouts and reduce collisions. 
This thesis has contributed in demonstrating the effectiveness of the advanced safety tool in diagnosing safety performance at roundabouts, and has showed the advantage of proactively identifying traffic conflicts and demonstrating safety issues at the roundabout. This proactive approach overcame the previous challenges in using collision-based evaluation techniques. It was applied on a two-day video data that overcame the long waiting time (e.g. two years) that is usually required to perform a collision-based study. It also overcame the use of low-quality collision records which tend to be highly subjective and usually fail to provide collision attributes. Each single conflict in the analysis includes details about the vehicle speed profile, the TTC value, and the location of the potential conflict point.

This thesis has been applied to diagnose the safety issues at a modern roundabout located at the University of British Columbia (UBC). Based on the results, several safety concerns have been found and summarized with proposed countermeasures listed in Table 6.1. These concerns have been determined by carefully reviewing and classifying the automated video output of the traffic conflicts that occurred in the roundabout. 
Table 6.1 Safety concerns found in the analysis and the proposed countermeasures.

Countermeasures

1. Confusion of the right of way between entering and circulating vehicles.

2. Frequent conflicts between exiting and circulating vehicles exiting approaches D and A.

3. Higher rate of rear-end conflicts at entry lanes than exit lanes especially at approach $\mathrm{C}$

4. Frequent conflicts between exiting vehicles at approach $B$ due to confusion over the proper negotiation in circulating lanes in front of approach $\mathrm{C}$.

5. Higher rate of vehicle-pedestrian conflicts at exit lanes than entry lanes.

6. Confusion of the vehicles on the proper negotiation of the roundabout resulting in some violations.
- Adding cross hatch marking and enlarging the road marking in front of the splitter island of approaches A and B to improve their visibility.

- Widening the separation lane lines between approaches $\mathrm{A}$ and $\mathrm{B}$, and between approach $\mathrm{A}$ and $\mathrm{D}$ to improve their visibility for both entering and circulating vehicles.

- Enlarging the yielding signs for entering vehicles to improve their yielding behaviour for circulating vehicles

- Adding pedestrian signs may help reduce pedestrian-vehicle and rear-end conflicts.

- $\quad$ Road marking around the central island and in front of approach D and C was suggested to be modified to reduce confusion about circulating in front of approach $\mathrm{C}$ and exiting negotiation at approach B.

- Providing pedestrian's signs for entering and exiting vehicles to improve their yielding behaviour for crossing pedestrians.

- Further education for drivers about right-of-way at roundabouts.

- Adding arrows at circulating lanes in front of approach A, B and D. Enlarging arrows at circulating lanes in front of approach $\mathrm{C}$. 


\begin{tabular}{|l|l|}
\hline $\begin{array}{l}\text { 7. Pedestrian violations in crossing } \\
\text { patterns especially at exit lanes. }\end{array}$ & $-\begin{array}{l}\text { Improving landscaping around pedestrian } \\
\text { crosswalks to improve directing pedestrians to } \\
\text { crosswalks and reduce jaywalking patterns. }\end{array}$ \\
$\begin{array}{l}\text { 8. Confusion on the accommodation } \\
\text { of cyclists in the mixed traffic lanes }\end{array}$ & $\begin{array}{l}\text { Further education for drivers about the } \\
\text { accommodation of cyclists in mixed traffic } \\
\text { roundabouts }\end{array}$ \\
\hline
\end{tabular}

These countermeasures were proposed to improve the safety level of the roundabout. The use of the automated safety tool in the diagnosis study has shown the value of the proactive identification of risky situations at the roundabout. The research proposes the potential engineering countermeasures using the Traffic Conflict Technique (TCT), rather than the traditional collision-based safety diagnosis approach.

There were several major lessons learned from this safety diagnostic process. One of the major lessons is the significance of identifying the focus road users group in early stages of the research. For example, if pedestrian safety was the main focus of this study, one or two more video cameras would be dedicated to capture the pedestrians' interactions at each approach of the roundabout. It also important to be more careful with the technical issues when recording the videos such as the camera position, zoom level, and angle. These issues affect the quality of the tracking that can make the feature tracking and event generation process require more manual filtering and post processing work.

One of the lessons learned is also related to the check of the geometric design elements at the roundabout as part of the safety diagnosis analysis. This check has been implemented on this research after the video analysis of the roundabout. However, it is better if this check has been 
implemented before the video analysis in order to expect the possible safety issues and focus on them at the safety diagnostic process.

Continuation of this work may include an after-treatment diagnosis analysis to validate the effectiveness of the countermeasures. A before-and-after study on the roundabout can be performed to validate the quantitative impact of the countermeasures on conflicts and accidents. The heat map produced for the roundabout conflicts per square meter can be correlated with a heat map for the accident that occurred at the roundabout. This has similarly been applied before in a large scale automated analysis of vehicle interactions and collision by Saunier, Sayed, and Ismail (2010). Conflict modification factors can be developed based on the objective proactive approach of the safety diagnosis. Future research can be performed on several roundabout designs to associate conflicts (rather than collisions) with a specific design parameter, as they are usually more frequent and easier to capture. Future work can also be performed to identify the ideal duration of a diagnosis study, as a two-days analysis might be (in some locations) less than what is required to significantly identify the adequate number of road users interactions.

In summary, this thesis presented an automated roundabout safety analysis to identify and remedy safety problems, and was applied for an in-service roundabout using the advanced UBC safety tool. Future work will include further advancements to the automated analysis approach with further developments, methodologies, and applications. 


\section{Bibliography}

National Cooperative Highway Research Program (NCHRP). (2010). Roundabout: An Informational Guide. Transportation Research Board of the National Academies. FHWA.

Google Maps. (2011). Retrieved April 01, 2011, from http://www.google.com/maps

Learn to Drive Smart. (2011). North Vancouver, British Columbia: Insurance Corporation of British Columbia.

Amundsen, F., \& Hydén, C. (1977). First Workshop on Traffic Conflicts. Institute of Transport Economics, Oslo, Norway.

Archer, J. (2004). Methods for the Assessment and Prediction of Traffic Safety at Urban Intersections and their Application in Micro-simulation Modelling. Stockholm, Sweden: Centre for Traffic Simulation Research, CTR.

Ashmead, D., Guth, D., Wall, R., Long, R., \& Ponchillia, P. (2005). Street Crossing by Sighted and Blind Pedestrians at a Modern Roundabout. Journal of Transportation Engineering, $131(11), 812-821$.

Atev, S. H., Massoud, O., Janardan, R., \& Papanikolopolous, N. (2005). A Vision-Based Approach to Collision Prediction at Traffic Intersections. IEEE Transactions on Intelligence Transportation, 6, 416-423.

Autey, J., Sayed, T., \& Zaki, M. H. (2011). Safety Evaluation of Right-Turn Smart Channels Using Automated Traffic Conflict Analysis. Accident Analysis and Prevention, 45, 120130. 
Baranowski, B. (2005). Pedestrian Crosswalk Signals at Roundabouts: Where Are They Applicable? Transportation Research E-Circular(E-C083), 15.

Beymer, D., McLauchlan, P., Coifman, B., \& Malik., J. (1997). A Real-Time Computer Vision System for Measuring Traffic Parameters. Conference on Computer Vision and Pattern Recognition (pp. 495-501). Washington, D.C.: IEEE Computer Society.

Brown, M. (1995). The Design of Roundabouts: State-of-the-Art Review. London: Trans. Res. Lab.

Chin, H.-C., \& Quek, S.-T. (1997). Measurement of Traffic Conflicts. Safety Science, 26(3), 169185.

Daniels, S., Brijs, T., Nuyts, E., \& Wets, G. (2009). Injury Crashes with Bicyclists at Roundabouts: Influence of Some Location Characteristics and the Design of Cycle Facilities. Journal of Safety Research, 40, 141-148.

Daniels, S., Nuyts, E., \& Wets, G. (2007). The Effects of Roundabouts on Traffic Safety for Bicyclists: An Observational Study. Accident Analysis and Prevention(40), 518-526.

Diah, J. M., Rahman, M. Y., Adnan, M. A., \& Atan, I. (2010). Weaving Section Flow Model at the Weaving Area of Malaysian Conventional Roundabout. Journal of Transportation Engineering, 136(8), 782-792.

FHWA. (2009). Manual on Uniform Traffic Control Devices for Streets and Highways. Washington, D.C.: U.S. Department of Transportation. 
Harkey, D. L., \& Carter, D. L. (2006). Observational Analysis of Pedestrian, Bicyclist, and Motorist Behaviors at Roundabouts in the United States. Transportation Research Board, $155-165$.

Hayward, J. (1968). Near-miss Determination through Use of a Scale of Danger. Highway Research Record, 384, 24-3.

Hu, W., Xiao, X., Xie, D., \& Tan, T. (2004). Traffic Accident Prediction Using 3-D ModelBased Vehicle Tracking. IEEE Transactions on Vehicular Technology, 53, 677-694.

Hu, W., Xie, D., \& Tan, T. (2004). A Hierarchical Self-Organizing Approach for Learning the Patterns of Motion Trajectories. IEEE Transactions on Neural Networks, 15(1), 135-144.

Hydén, C. (1987). The Development of a Method for Traffic Safety Evaluation: The Swedish Traffic.

Insurance Institute for Highway Safety. (2008). Unpublished analysis of GES and FARS 2006 Database. Arlington, VA: IIHS.

Ismail, K. A. (2010). Application of Computer Vision Techniques for Automated Road Safety Analysis and Traffic Data Collection. Vancouver: The University of British Columbia.

Ismail, K., Sayed, T., Saunier, N., \& Lim, C. (2009). Automated Analysis of Pedestrian-Vehicle Conflicts Using Video Data. Transportation Research Record: Journal of the Transportation Research Board(2140), 44-54.

Jordan, P. (1985). Pedestrians and Cyclists at Roundabouts. Third National Local Government Engineering Conference. Melbourne, AU: Institution of Engineers. 
Kamijo, S., Matsushita, Y., Ikeuchi, K., \& Sakauchi, M. (2000). Traffic Monitoring and Accident Detection at Intersections. IEEE Conference on Intelligence Transportation Systems, 1, pp. 108-118.

Kastrinaki, V., Zervakis, M., \& Kalaitzakis, K. (2003, April). A Survey of Video Processing Techniques for Traffic Applications. Image and Vision Computing, 21(4), 359-381.

Kim, Z. (2008). Real Time Object Tracking based on Dynamic Feature Grouping with Background Subtraction. IEEE Computer Vision and Pattern Recognition, (pp. 1-8). Alaska.

Laureshyn, A., \& Ardo., H. (2006). Automated Video Analysis as a Tool for Analysing Road Safety Behaviour. ITS World Congress. London.

Lenters, M. S. (2005). Safety Auditing Roundabouts. Transportation Research E-Circular(EC083), 20.

Lu, G. (.-Z., Guan, F., \& Noyce, D. A. (2010). Multimodal Accessibility of Modern Roundabouts: Intelligent Management System Versus Common Signalization Scheme. Transportation Research Record: Journal of the Transportation Research Board(2183), $103-119$

Mandavilli, S., McCartt, A. T., \& Retting, R. A. (2009). Crash Patterns and Potential Engineering Countermeasures at Maryland Roundabouts. Traffic Injury Prevention, 10(1), 44-50.

Mauro, R., \& Cattani, M. (2010). Potential Accident Rate of Turbo-Roundabouts. 4th International Symposium on Highway Geometric Design, (p. 16). 
Montella, A. (2007). Roundabout In-Service Safety Reviews: Safety Assessment Procedure. Transportation Research Record: Journal of the Transportation Research Board(2019), $40-50$.

Montella, A. (2010). Analysis of Crash Contributory Factors at Urban Roundabouts. Transportation Research Board 89th Annual Meeting, (p. 18).

Montella, A. (2011). Identifying crash contributory factors at urban roundabouts and using association rules to explore their relationships to different crash types. Accident Analysis \&amp; Prevention, 43(4), 1451-1463.

Perkins, R., \& Harris, J. (1968). Traffic Conflict Characteristics: Accident Potential at Intersections. Highway, 225, 35-43.

Road Safety Directorate. (2007). Intersection-Related Crashes in Canada: Driver Characteristics and Casualty Trends. Transport Canada.

Rodegerdts, L., Blogg, M., Wemple, E., Myers, E., Kyte, M., Dixon, M., et al. (2007). NCHRP Report 572: Roundabouts in the United States. Transportation Research Board of the National Academies.

Sakshaug, L., Laureshyn, A., Svensson, A., \& Hyden, C. (2010). Cyclists in roundabouts-Different design solutions. Accident Analysis \& Prevention, 42(4), 1338-1351.

Saunier, N., \& Sayed, T. (2007). Automated road safety analysis using video data. Transportation Research Record: Journal of the Transportation Research Board(2019), $57-64$. 
Saunier, N., \& Sayed, T. (2008). A Probabilistic Framework for Automated Analysis of Exposure to Road. Transportation Research Record: Journal of the Transportation Research Board, 2083, 96-104.

Saunier, N., \& Sayed, T. A. (2006). A Feature-Based Tracking Algorithm for Vehicles in Intersections. 3rd Canadian Conference on Computer and Robot Vision. Quebec City, Canada: IEEE.

Saunier, N., Sayed, T., \& Ismail, K. (2010). Large scale automated analysis of vehicle intereactions and collisions. Journal of the Transportation Research Board, 2147, 42-50.

Sayed, T., \& Zein, S. (1999). Traffic conflict standards for Intersections. Transportation Planning and Technology(22:4), 309-323.

Sayed, T., Brown, G., \& Navin, F. (1994). Simulation of traffic conflicts at unsignalized intersections with TSC-Sim. Accident Analysis \& Prevention, 26(5), 593-607.

Sayed, T., deLeur, P., \& Sawalha, Z. (2004). Evaluating the Insurance Corporation of British Columbia Road-Safety Improvement Program. Journal of the Transportation Research Board, 1865, 57-63.

Schoon, C. C., \& Minnen, J. v. (1993). Accidents on Roundabouts II. SWOV Institute for Road Safety Research, Publication Reference R-93-16.

Schroeder, B. J., \& Rouphail, N. M. (2007). Framework for Evaluating Pedestrian-Vehicle Interactions at Unsignalized Crossing Facilities in a Microscopic Modeling Environment. 86th Annual Meeting of the Transportation Research Board. Washington, D.C. 
Schroeder, B. J., Rouphail, N. M., \& Hughes, R. G. (2009). Working Concept of Accessibility: Performance measures for the Usability of Crosswalks by Pedestrian with Vision Impairment. Transportation Research Board: Journal of the Transportation Research $\operatorname{Board}(2140), 103-110$.

Skene, M., Jacobson, M., Havercroft, D., \& Boan, J. (2010). Considerations for Accommodating Visually Impaired Pedestrians at Roundabouts. ITE 2010 Annual Meeting and Exhibit, (p. $13)$.

Songchitruksa, P. (2004). Innovative non-crash-based safety estimation: an extreme value theory approach. $\mathrm{PhD}$ Thesis, Purdue University.

Songchitruksa, P., \& Tarko, A. (2006). The Extreme Value Theory Approach to Safety Estimation. Accident, 38(4), 811-822.

Svensson, Å., \& Hydén, C. (2006). Estimating the severity of safety related behaviour. Accident Analysis \& Prevention.

Tumber, C. (1997). Review of Pedestrian Safety at Roundabouts. Road Safety Department Melbourne. AU: Vic Roads.

Zheng, D., Qin, X., \& Noyce, D. A. (2010). Negotiation-Based Conflict Exposure Methodology in Roundabout Crash Pattern Analysis. Transportation Research Board 89th Annual Meeting, (p. 17). 


\section{Appendices}

\section{Appendix A: Vehicle Counts}

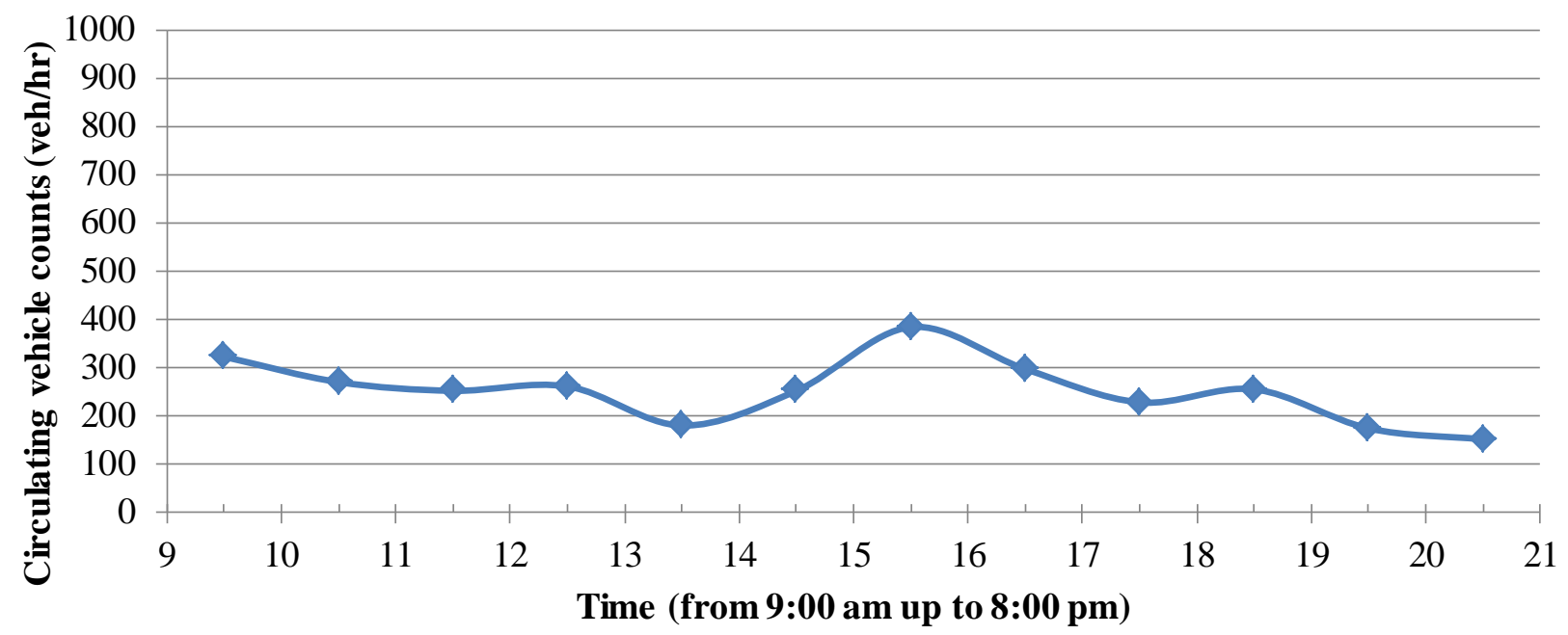

$\leadsto$ Circulating lane

(a)

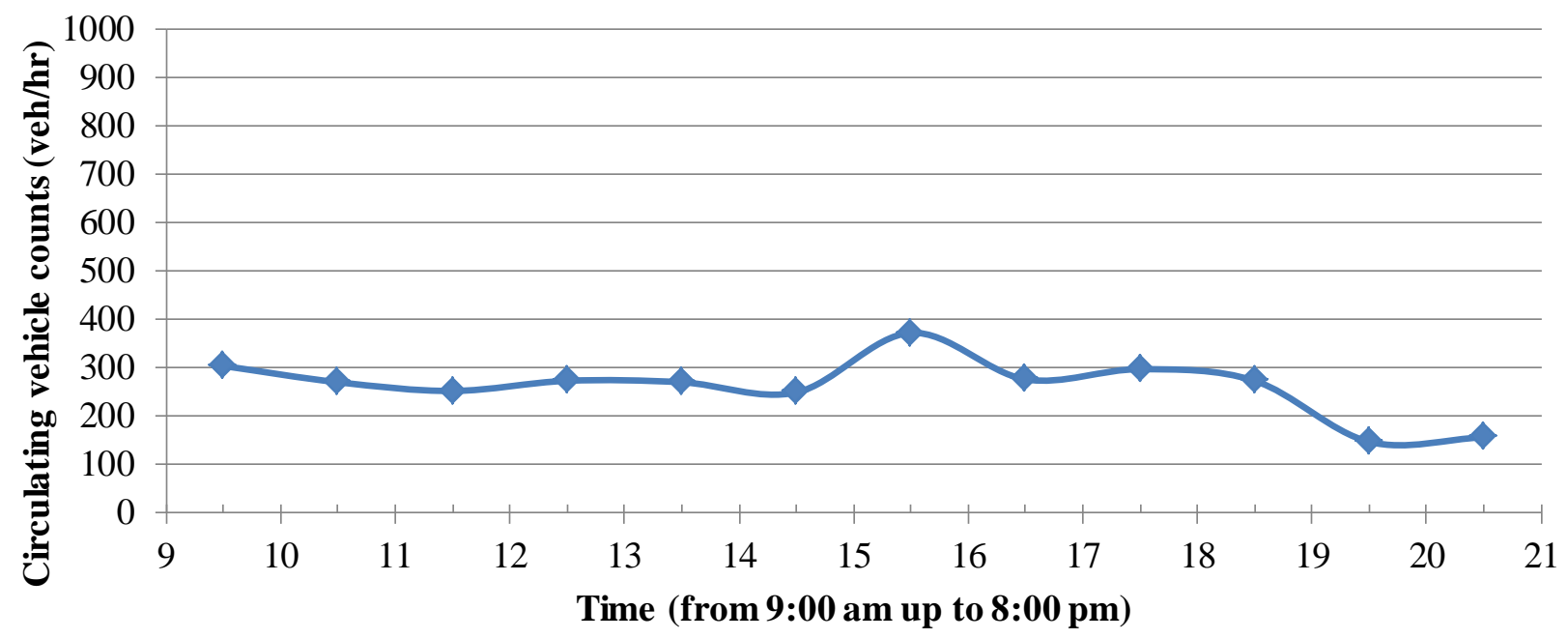

$\leadsto$ Circulating lane

(b)

Figure A.1 Circulating vehicle volumes for approach A from 9:00 am to $8 \mathrm{pm}$ a) Day one, and b) Day two 


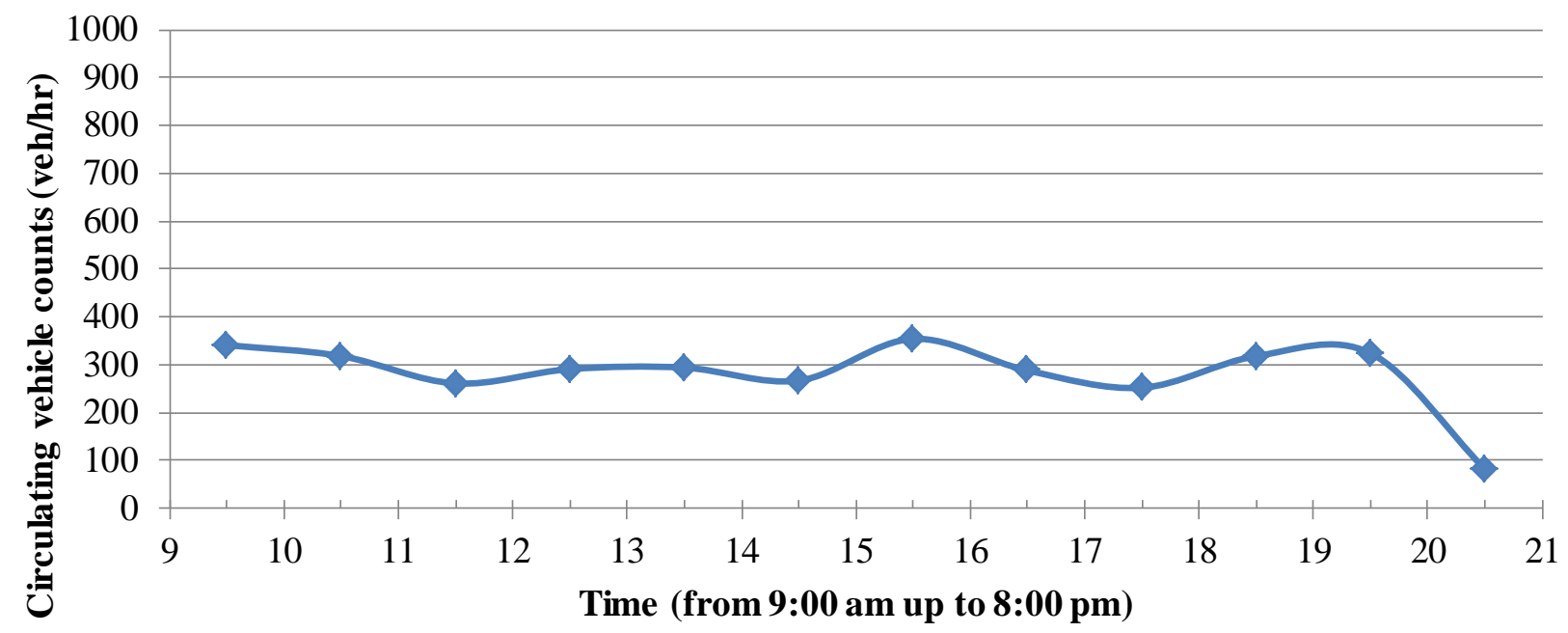

$\leadsto$ Circulating inner lane

(a)

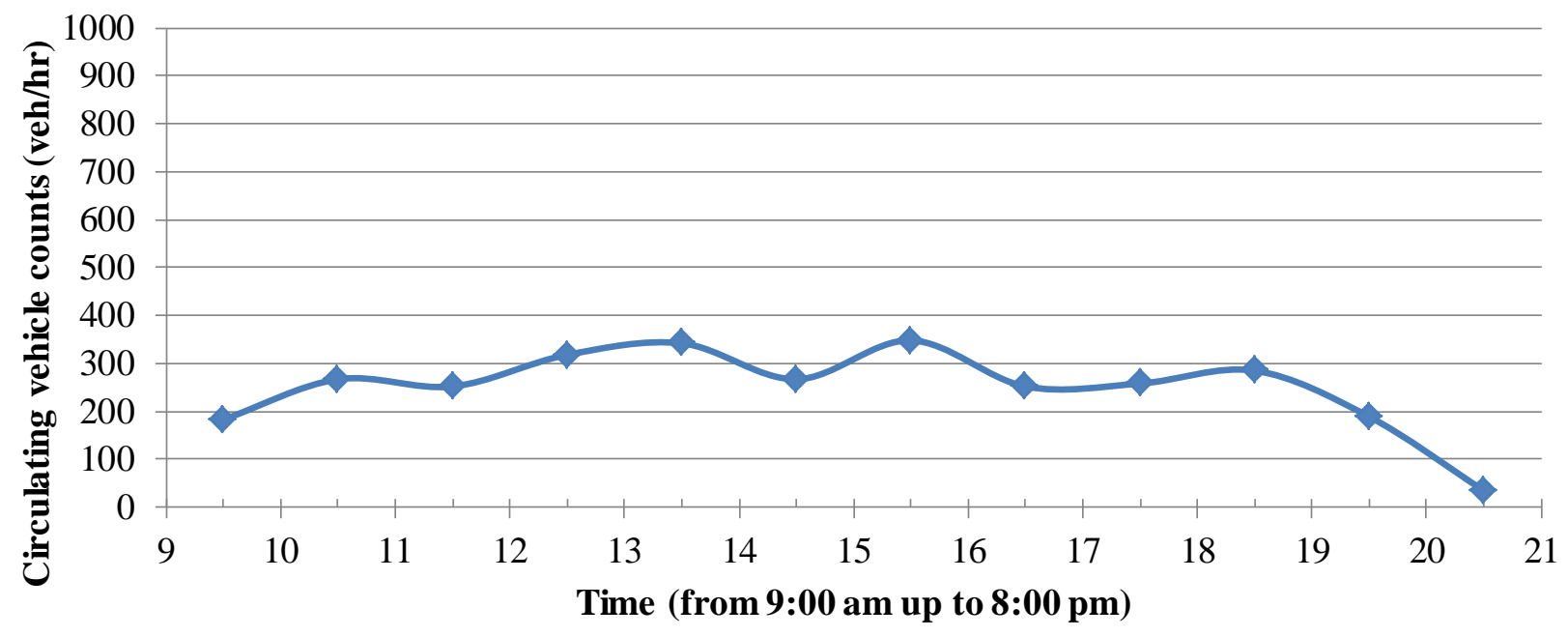

$\leadsto$ Circulating inner lane

(b)

Figure A.2 Circulating vehicle volumes for approach B from 9:00 am to 8 pm a) Day one, and b) Day two 


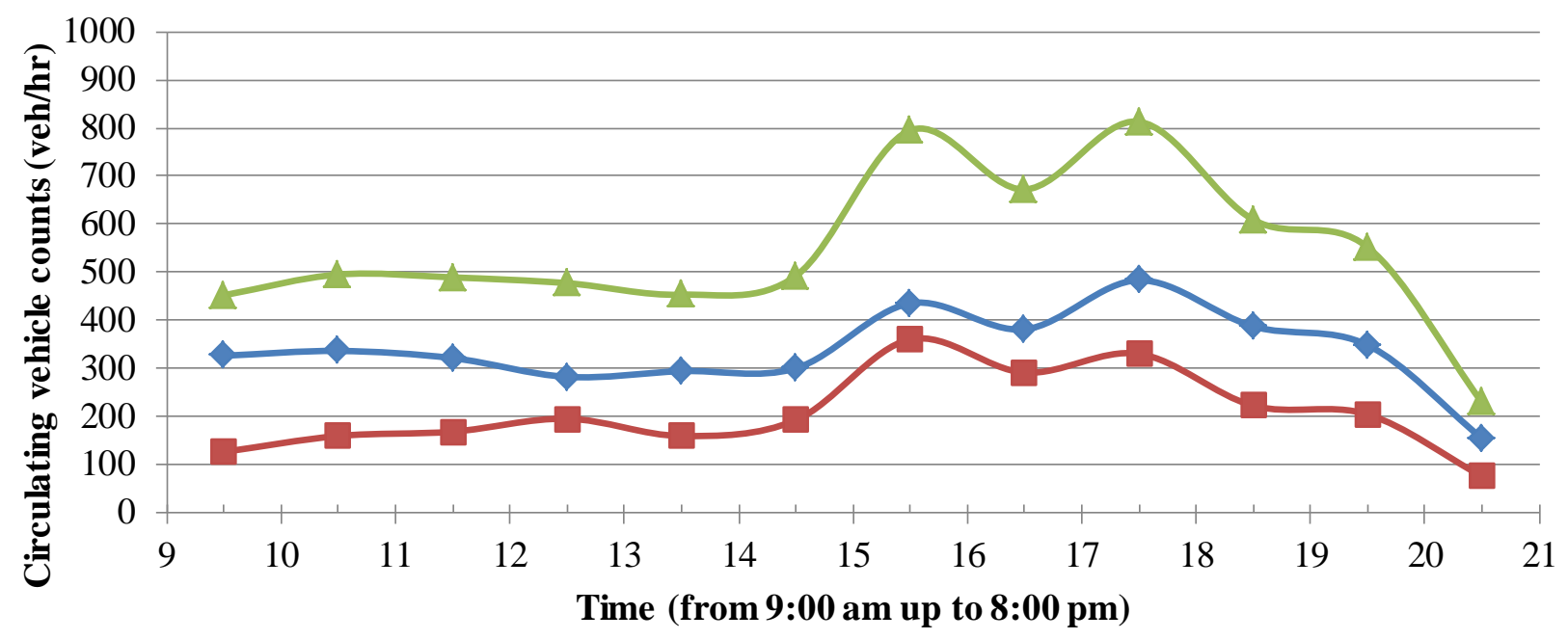

$\leadsto$ Circulating inner lane $\rightarrow$ Circulating outer lane $\quad \rightarrow$ Total Circulating

(a)

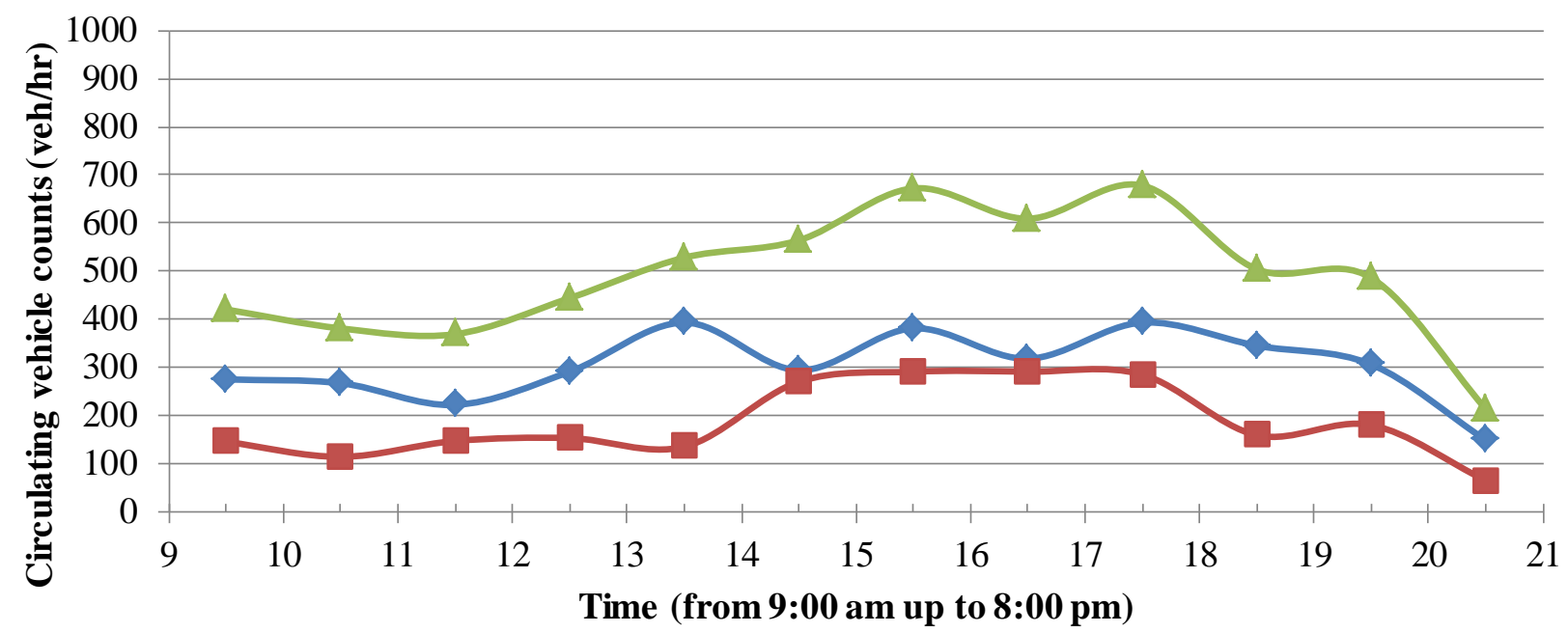

$\leadsto$ Circulating inner lane $\rightarrow-$ Circulating outer lane $\rightarrow$ Total Circulating

(b)

Figure A.3 Circulating vehicle volumes for approach C from 9:00 am to $8 \mathrm{pm}$ a) Day one, and b) Day two 


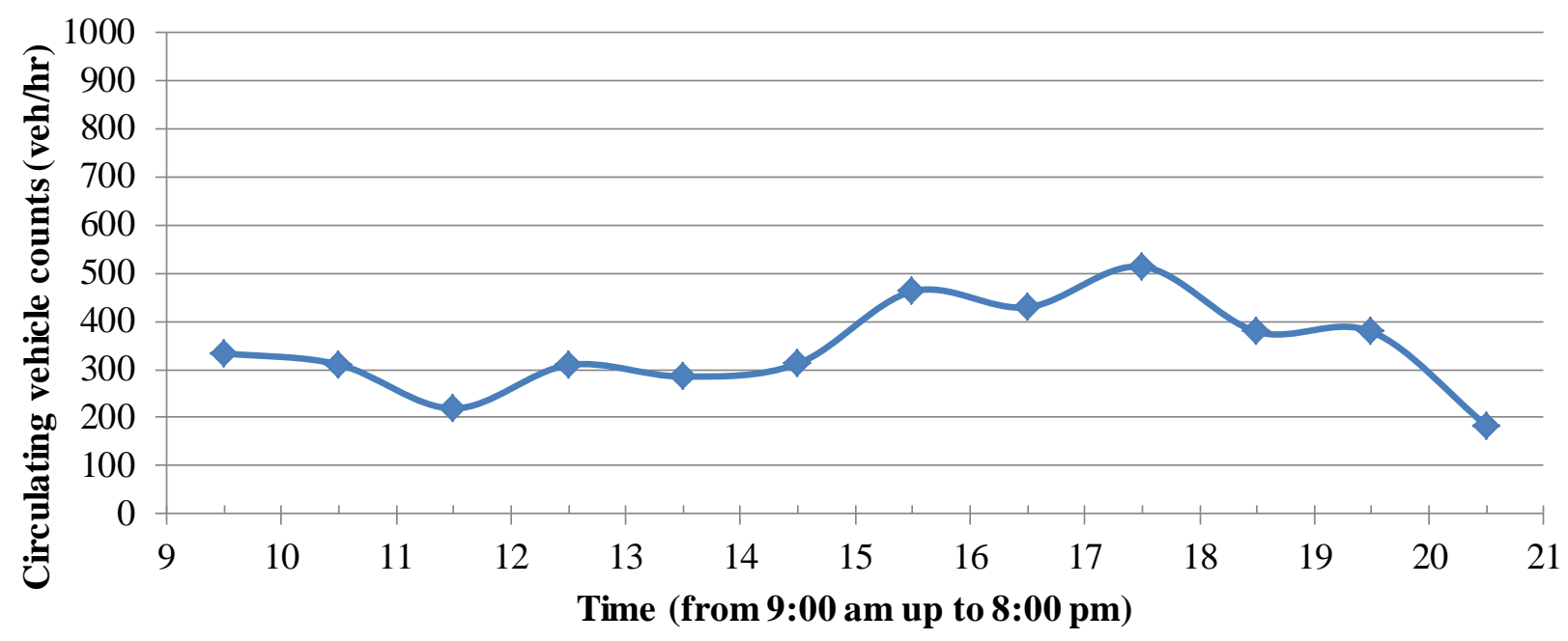

$\leadsto$ Circulating inner lane

(a)

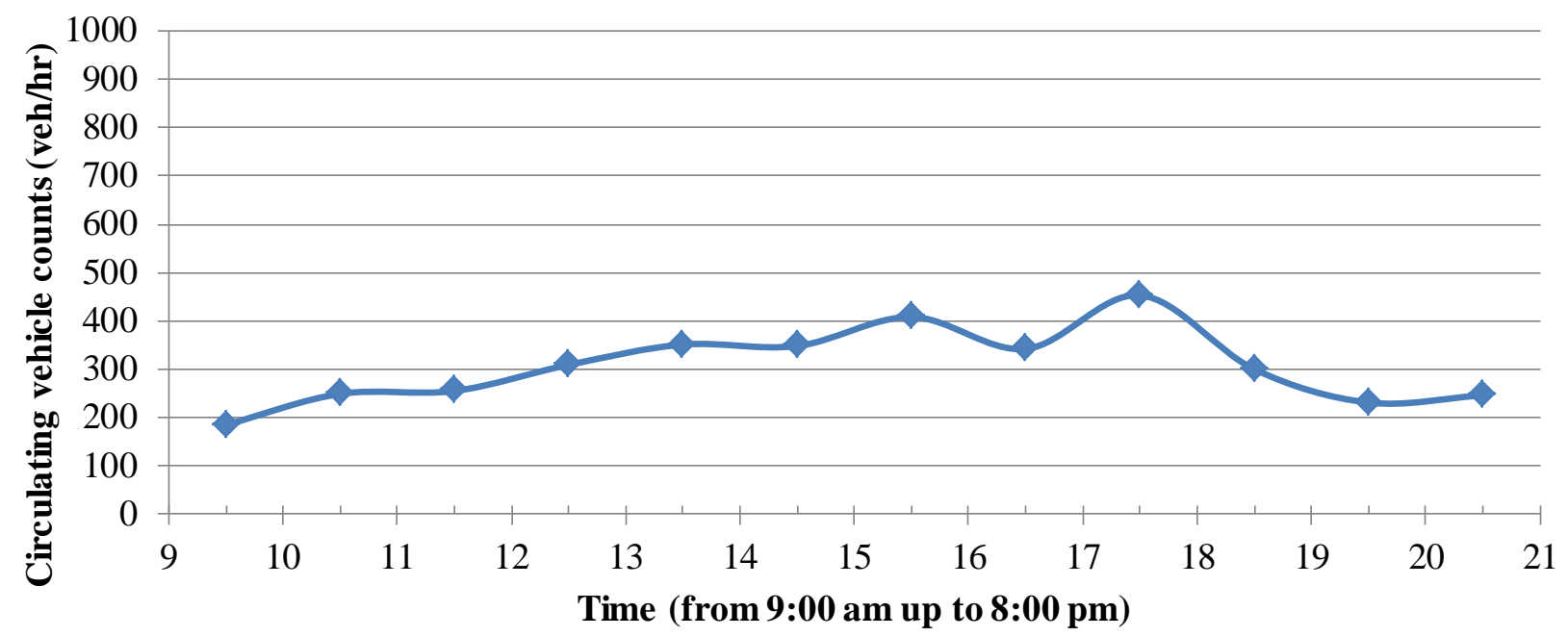

$\leadsto$ Circulating inner lane

(b)

Figure A.4 Circulating vehicle volumes for approach D from 9:00 am to $8 \mathrm{pm}$ a) Day one, and b) Day two 


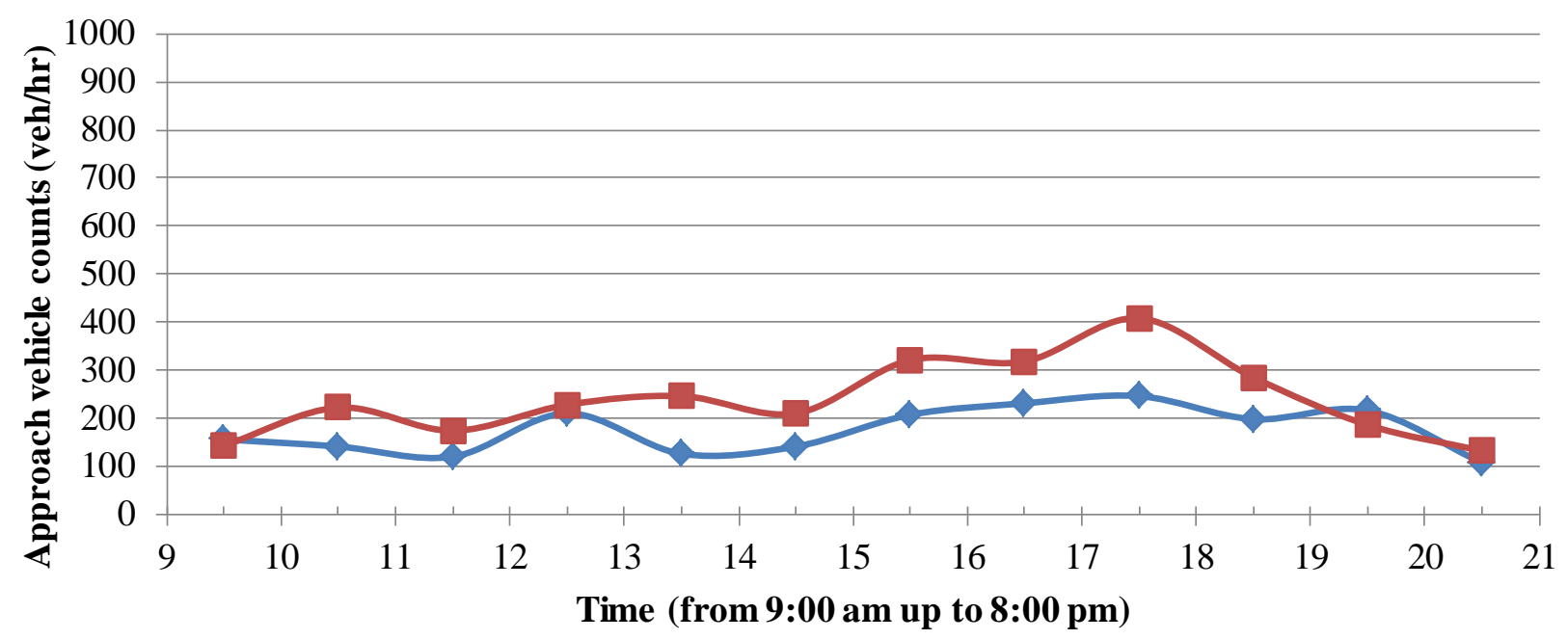

$\multimap$ Entering vehicles lane $1 \quad \neg$ Entering vehicles lane 2

(a)

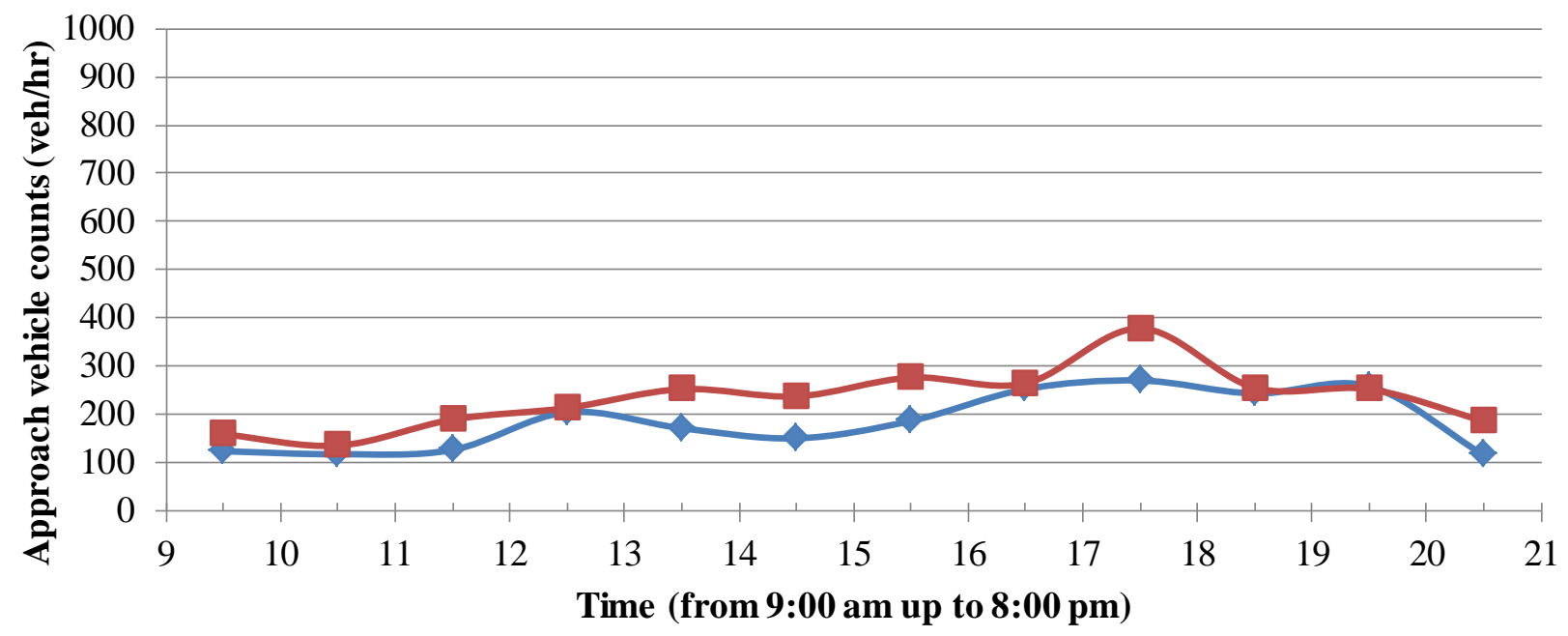

$\multimap$ Entering vehicles lane $1 \quad$ Entering vehicles lane 2

(b)

Figure A.5 Entering vehicle counts for approach A (lane one and two) from 9:00 am to 8:00 pm a) Day one, and b) Day two 


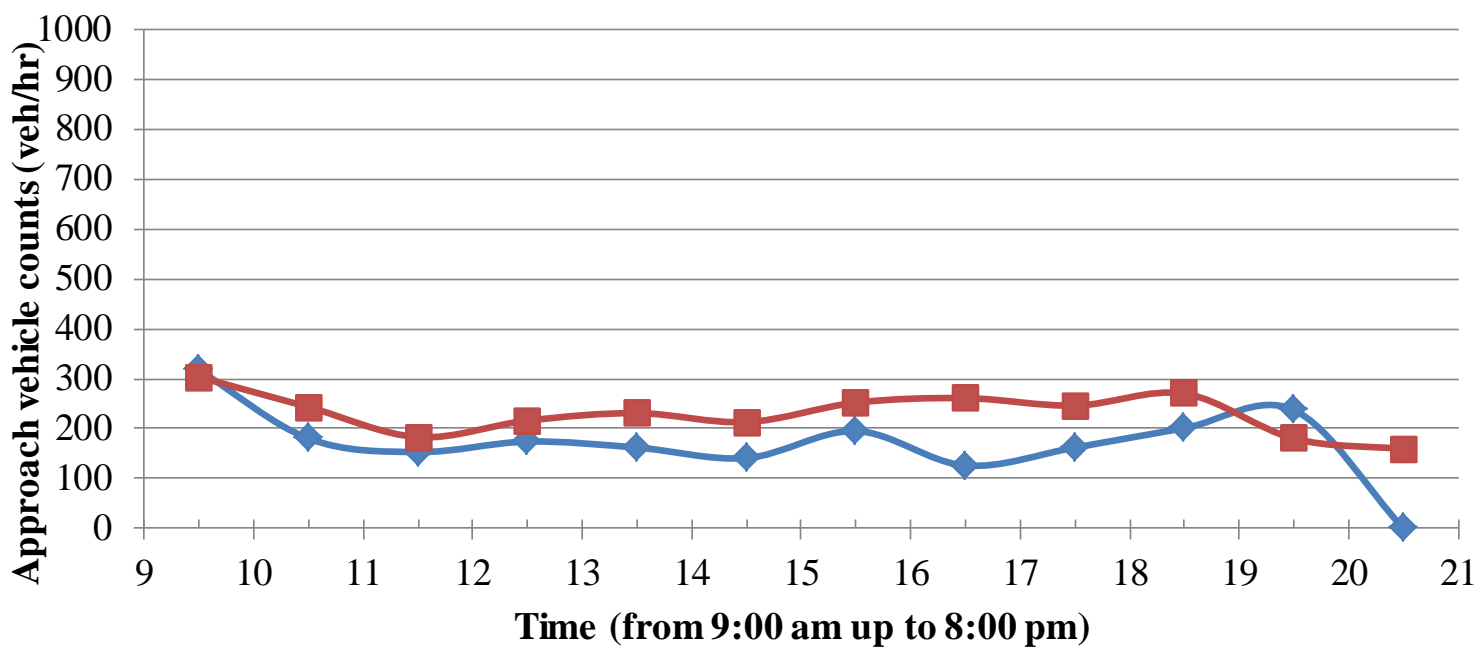

$\neg$ Exiting vehicles lane $1 \rightarrow-$ Exiting vehicles lane 2

(a)

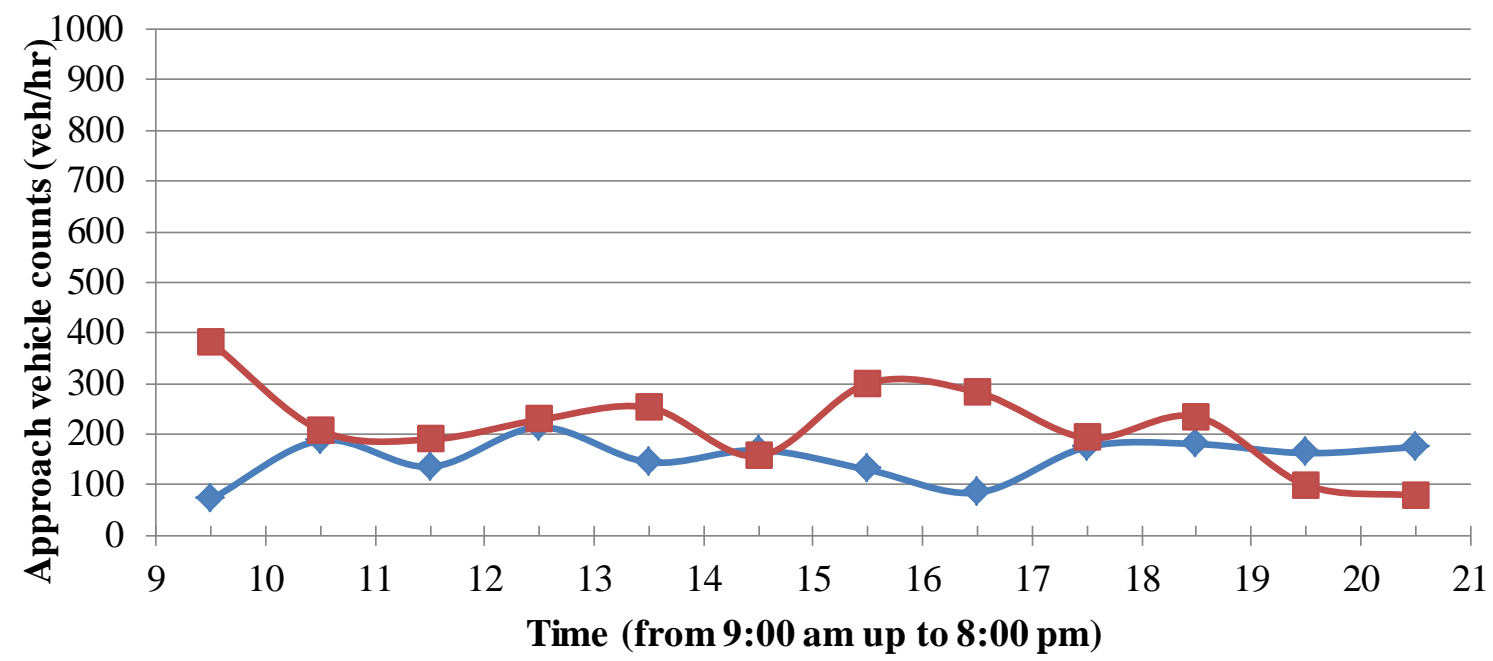

$\multimap$ Exiting vehicles lane $1 \multimap-$ Exiting vehicles lane 2

(b)

Figure A.6 Exiting vehicle counts for approach A (lane one and two) from 9:00 am to 8:00 pm a) Day one, and b) Day two 


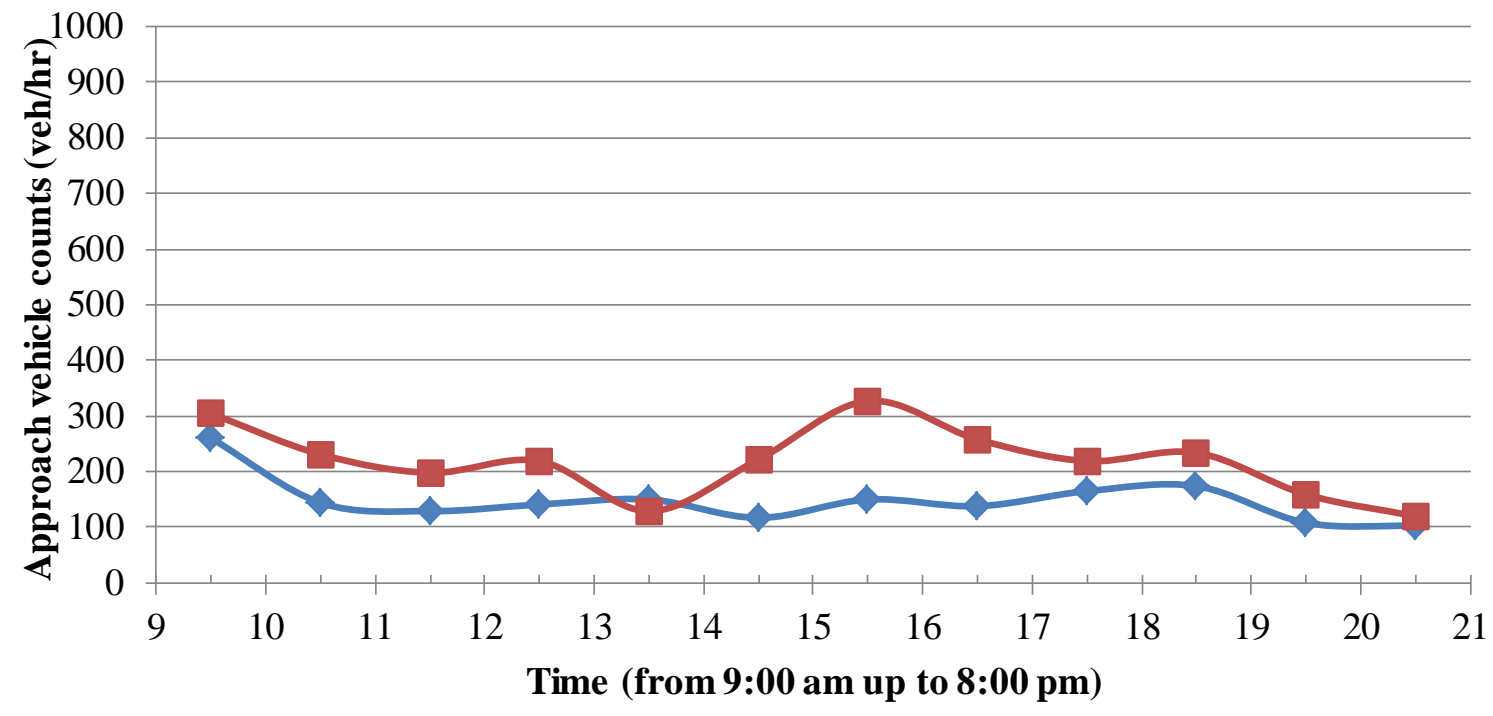

$\neg$ Entering vehicles lane $1 \rightarrow$ Entering vehicles lane 2

(a)

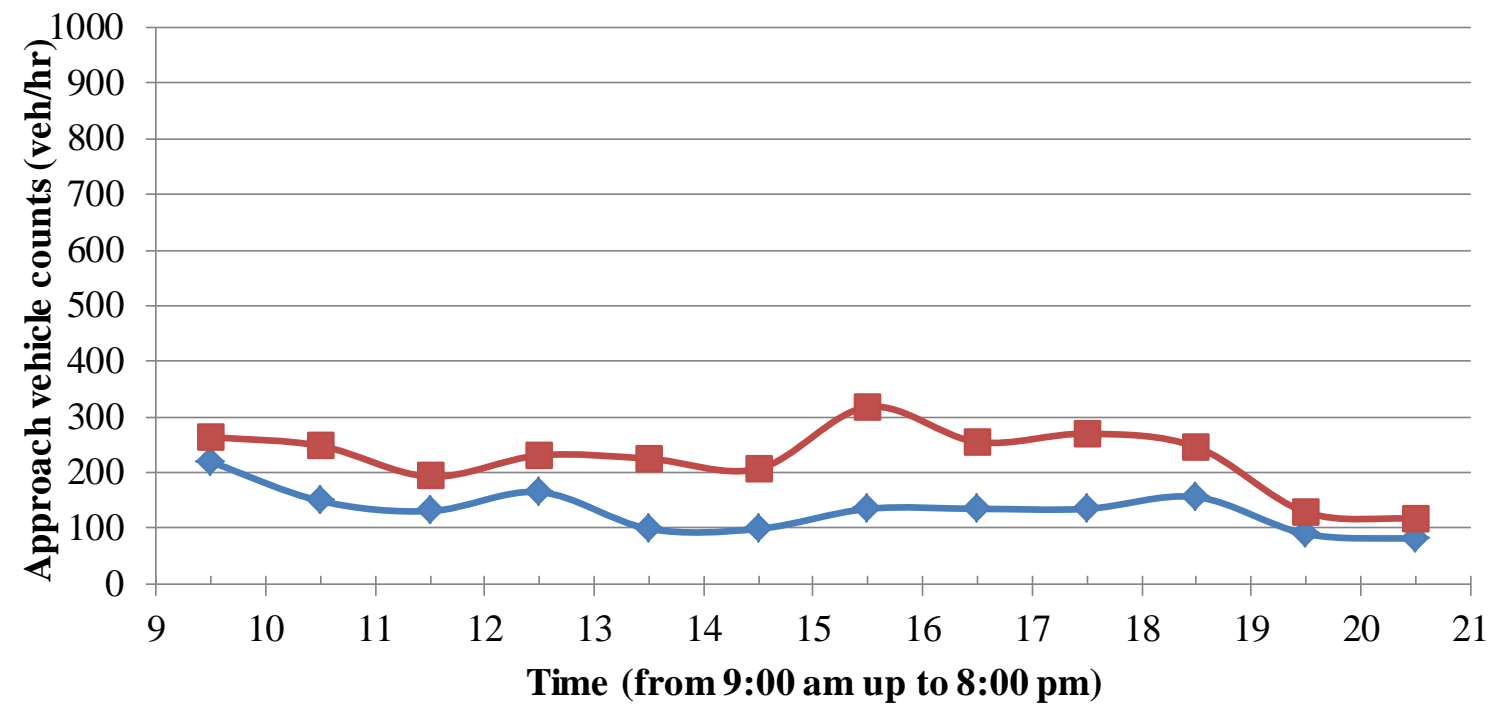

$\leadsto$ Entering vehicle lane $1 \rightarrow$ Entering vehicle lane 2

(b)

Figure A.7 Entering vehicle counts for approach B (lane one and two) from 9:00 am to 8:00 pm a) Day one, and b) Day two 


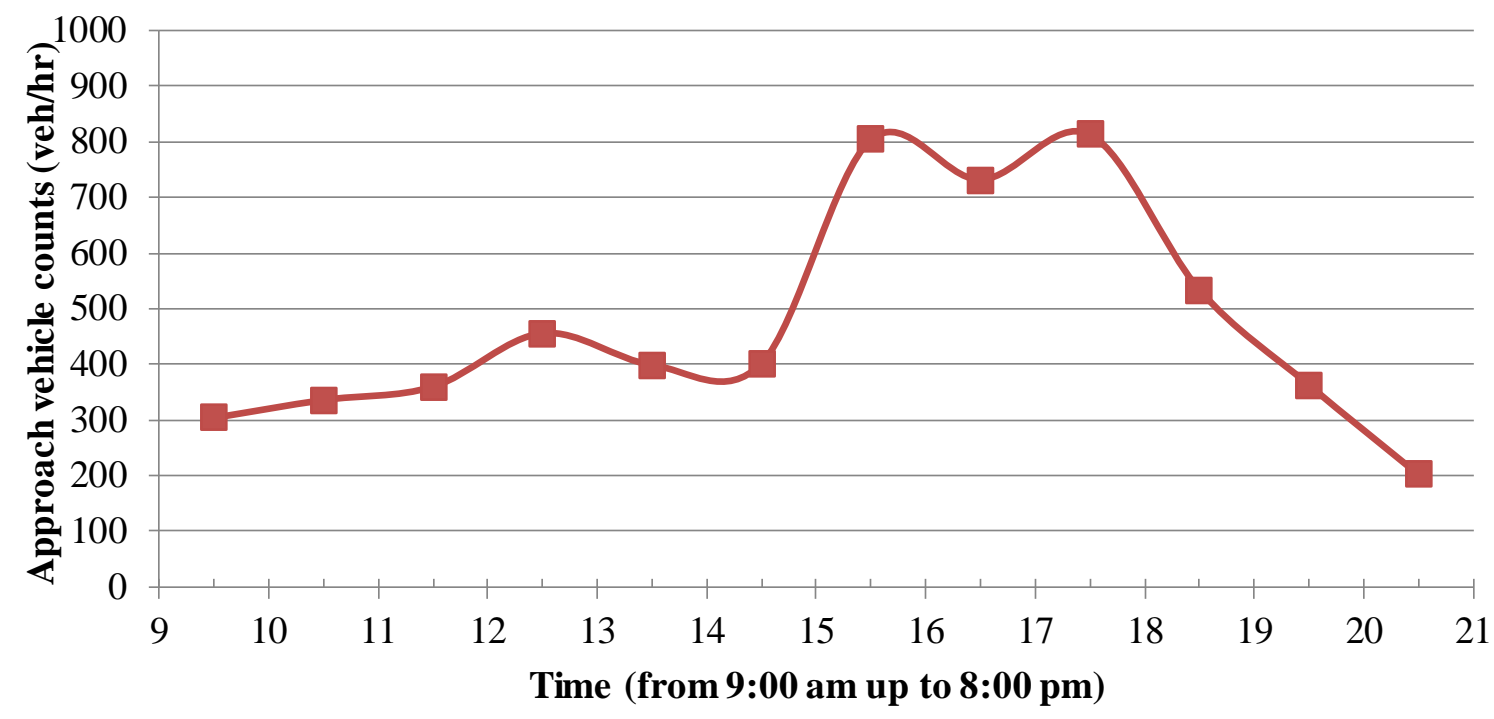

- Existing vehicles

(a)

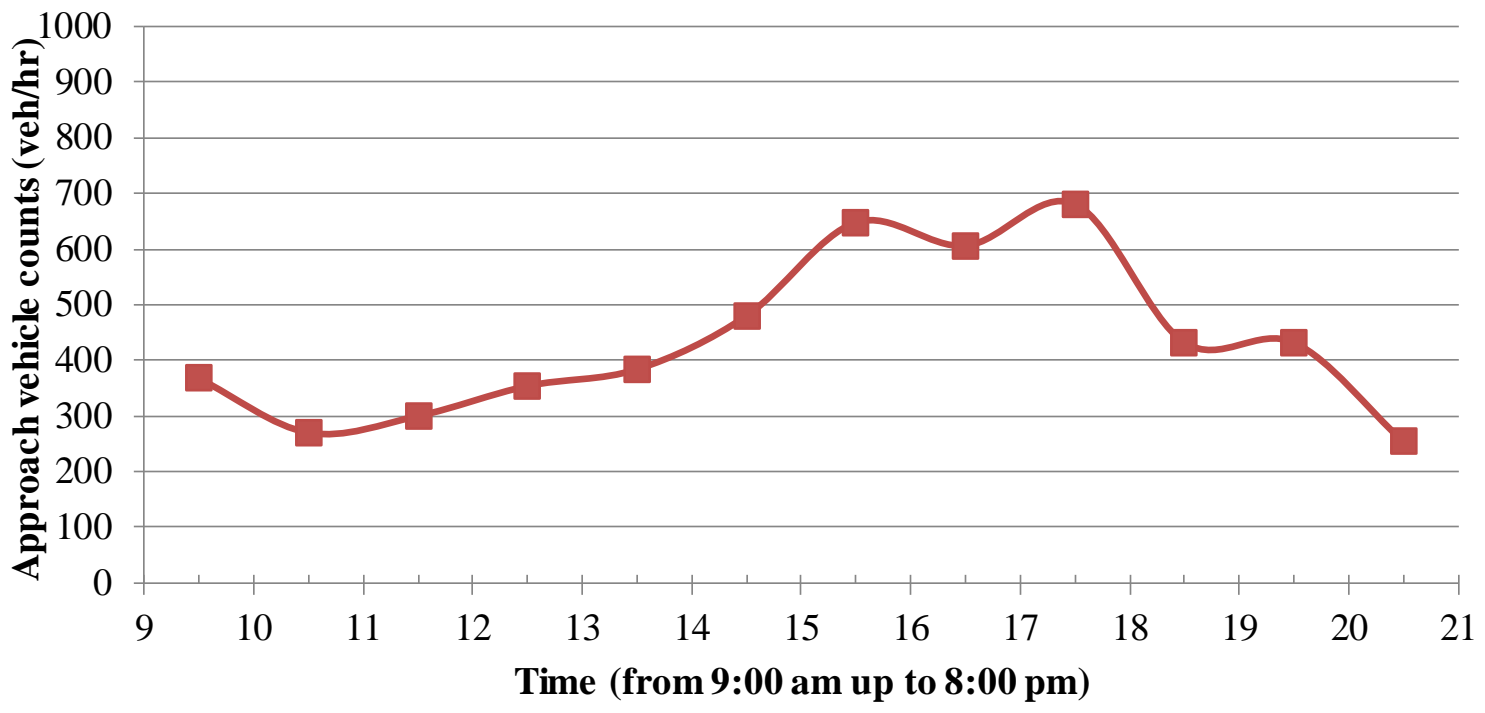

- Existing vehicles

(b)

Figure A.8 Exiting vehicle counts for approach B (lane one and two) from 9:00 am to 8:00 pm a) Day one, and b) Day two 


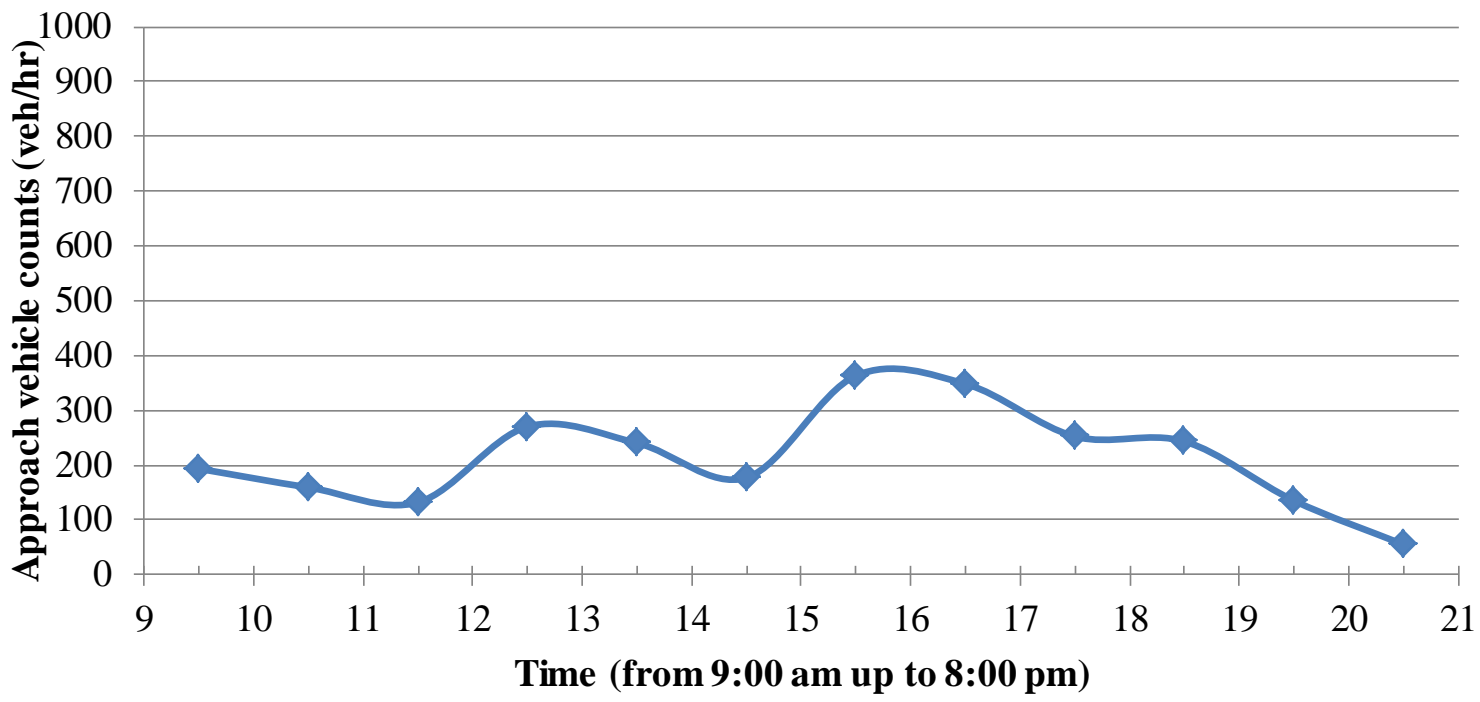

$\sim$ Entering vehicles

(a)

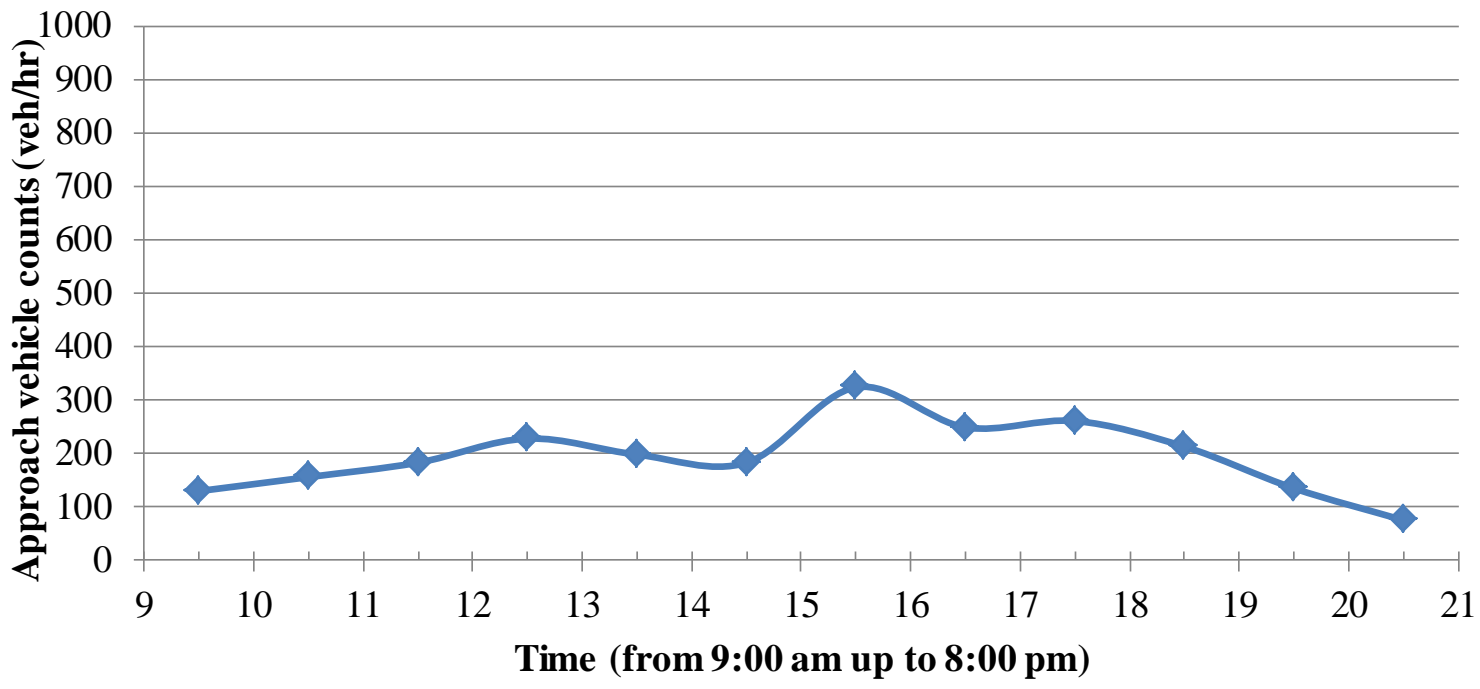

$\multimap$ Entering vehicles

(b)

Figure A.9 Entering vehicle counts for approach C lane 1 from 9:00 am to 8:00 pm a) Day one, and b) Day two 


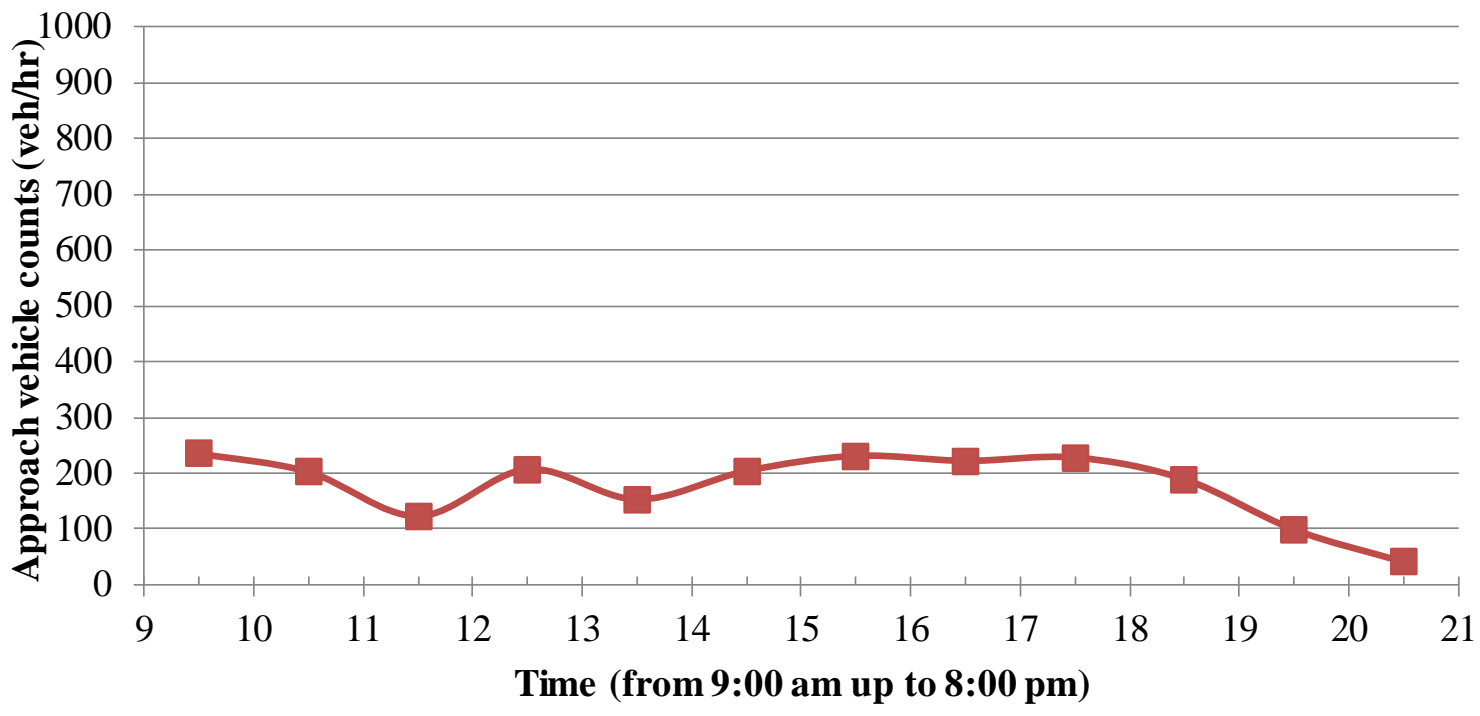

$\dashv$-Existing vehicles

(a)

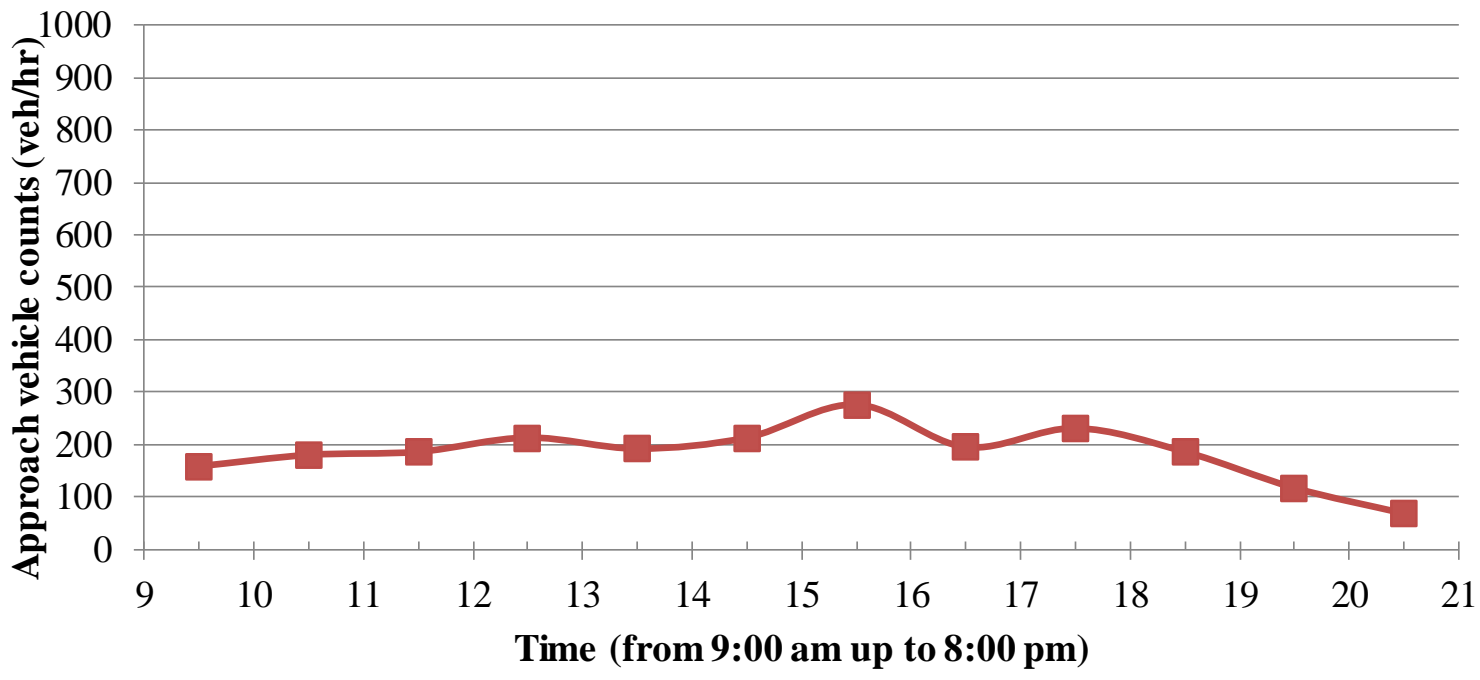

Existing vehicles

(b)

Figure A.10 Exiting vehicle counts for approach $C$ lane 1 from 9:00 am to 8:00 pm a) Day one, and b) Day two 


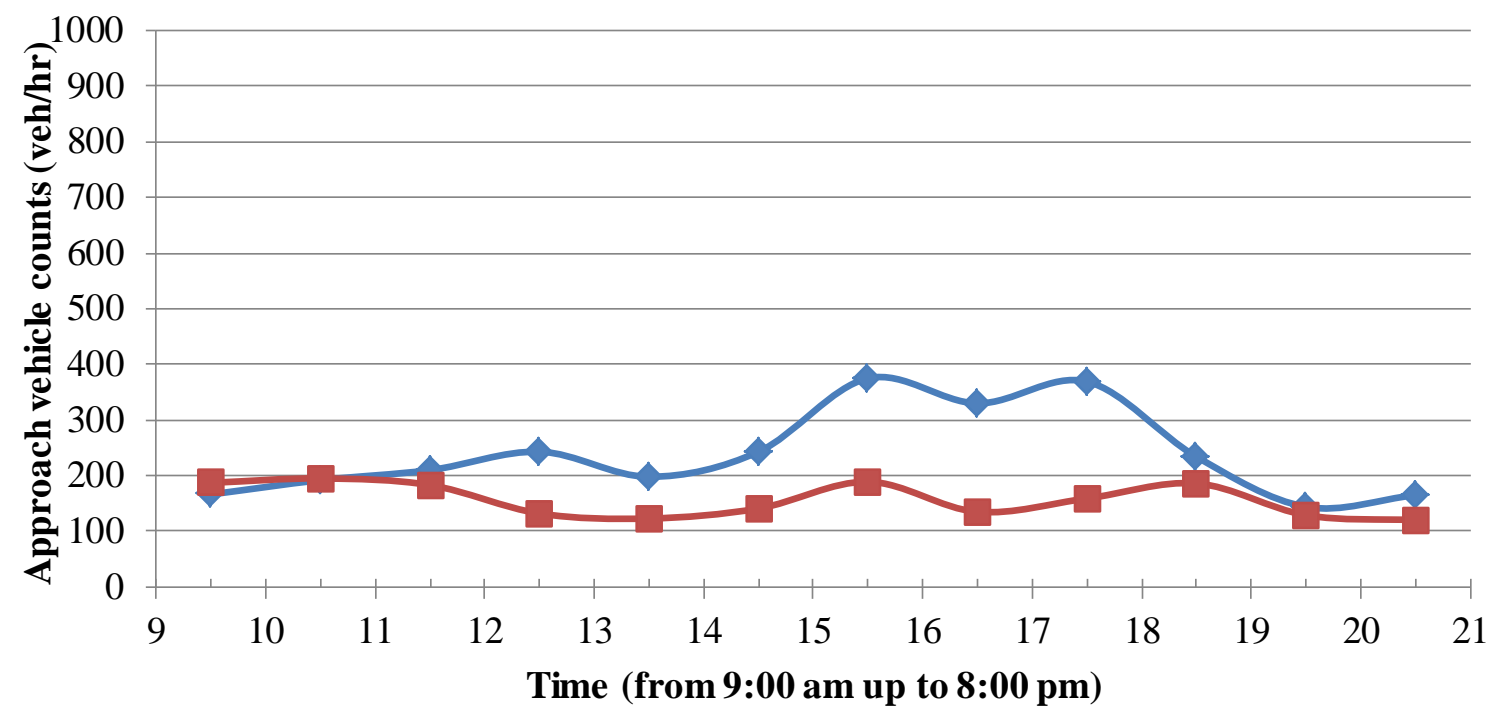

$\neg$ Entering vehicles lane $1 \rightarrow$ Entering vehicles lane 2

(a)

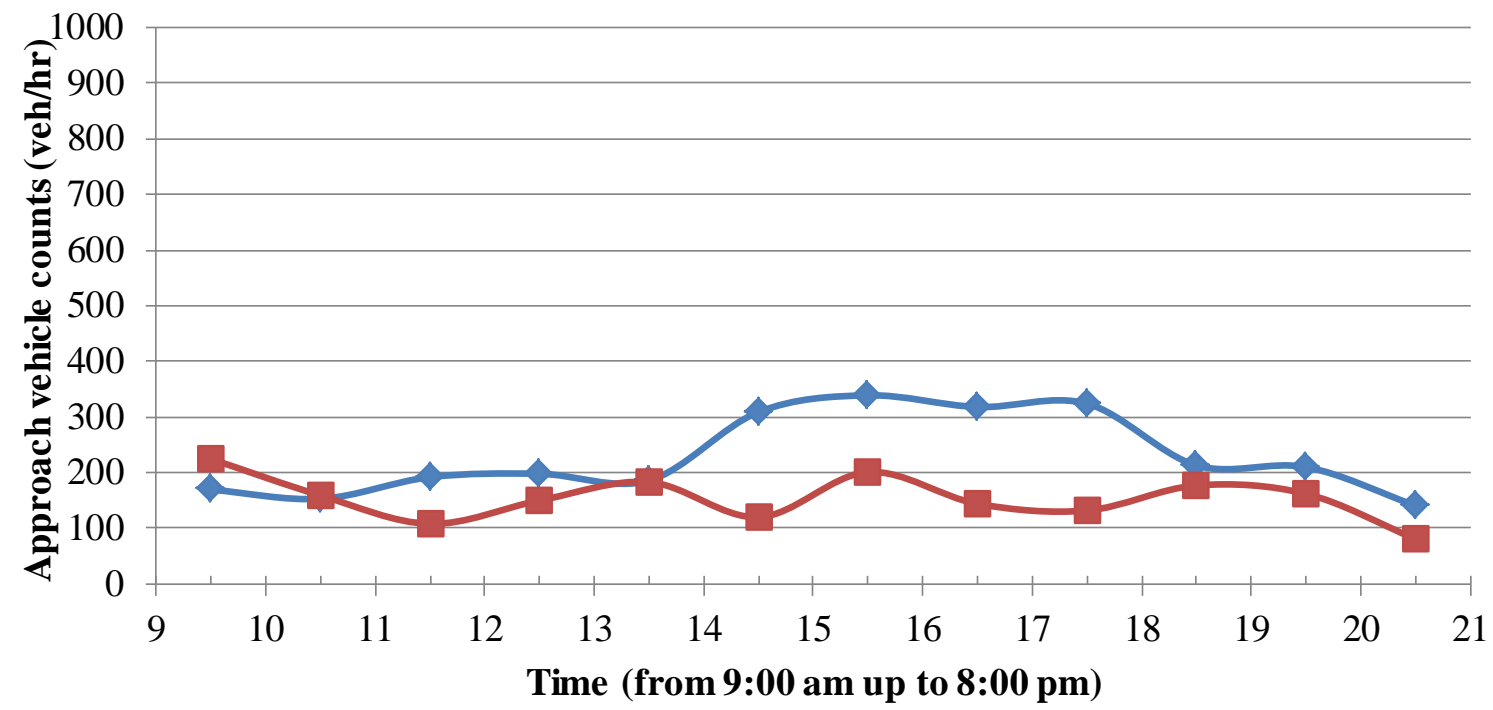

$\neg$ Entering vehicles lane $1 \rightarrow$ Entering vehicles lane 2

(b)

Figure A.11 Entering vehicle counts for approach D lane 1 from 9:00 am to 8:00 pm a) Day one, and b) Day two 


\section{Appendix B: Pedestrian Counts}

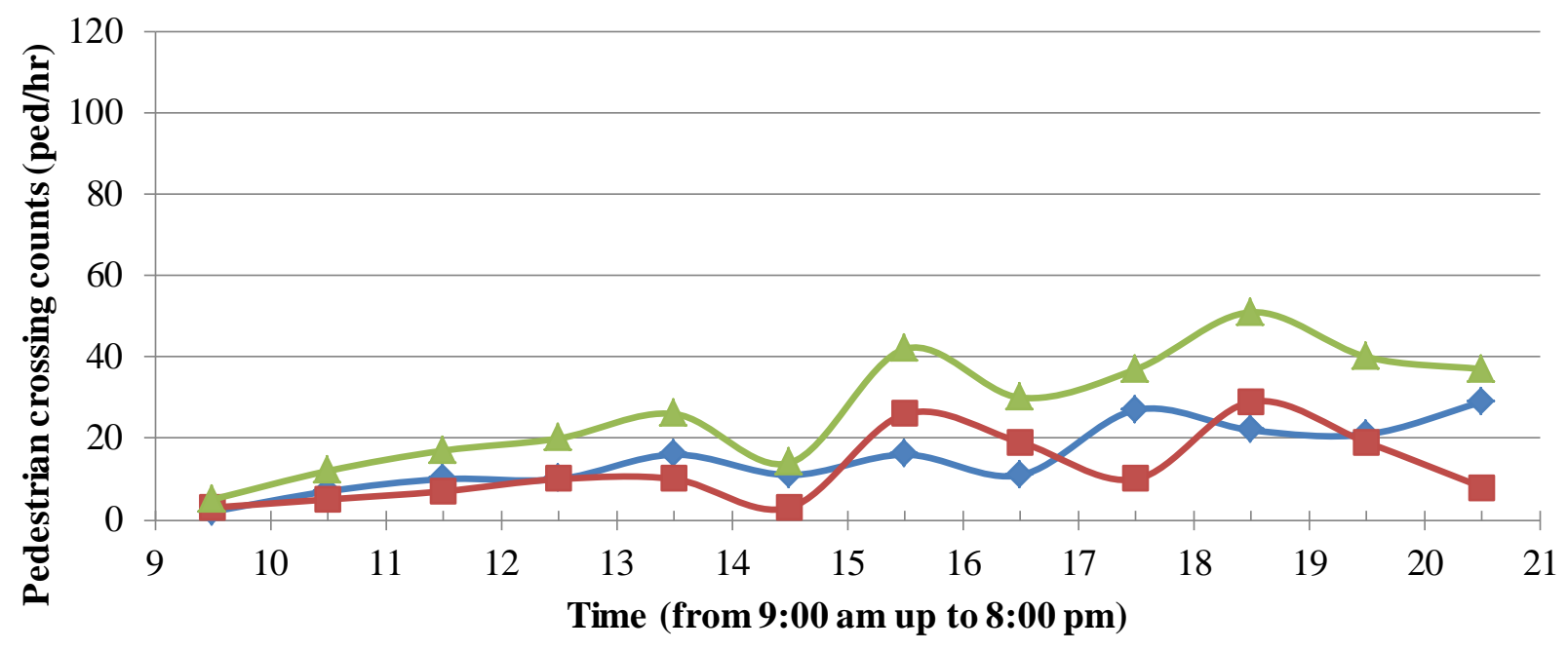

$\sim$ Crossing pedestrians from entry lane - Crossing pedestrians from exit lane - All crossing pedestrians

(a)

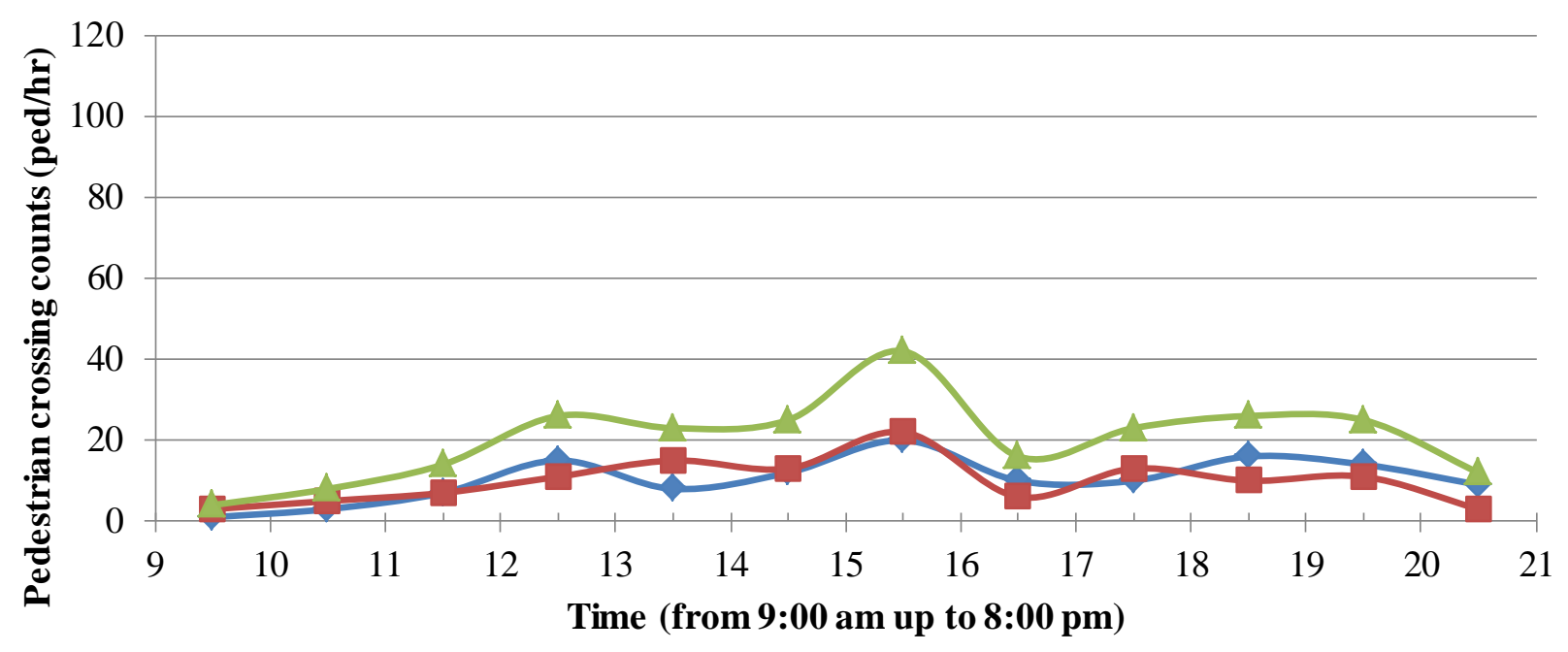

$\neg$ Crossing pedestrians from entry lane $\neg$ Crossing pedestrians from exit lane $\longrightarrow$ All crossing pedestrians

(b)

Figure B.1 Crossing pedestrians at approach A beginning from entry lane and from exit lane from 9:00 am to 8:00 pm a) Day one, and b) Day two 


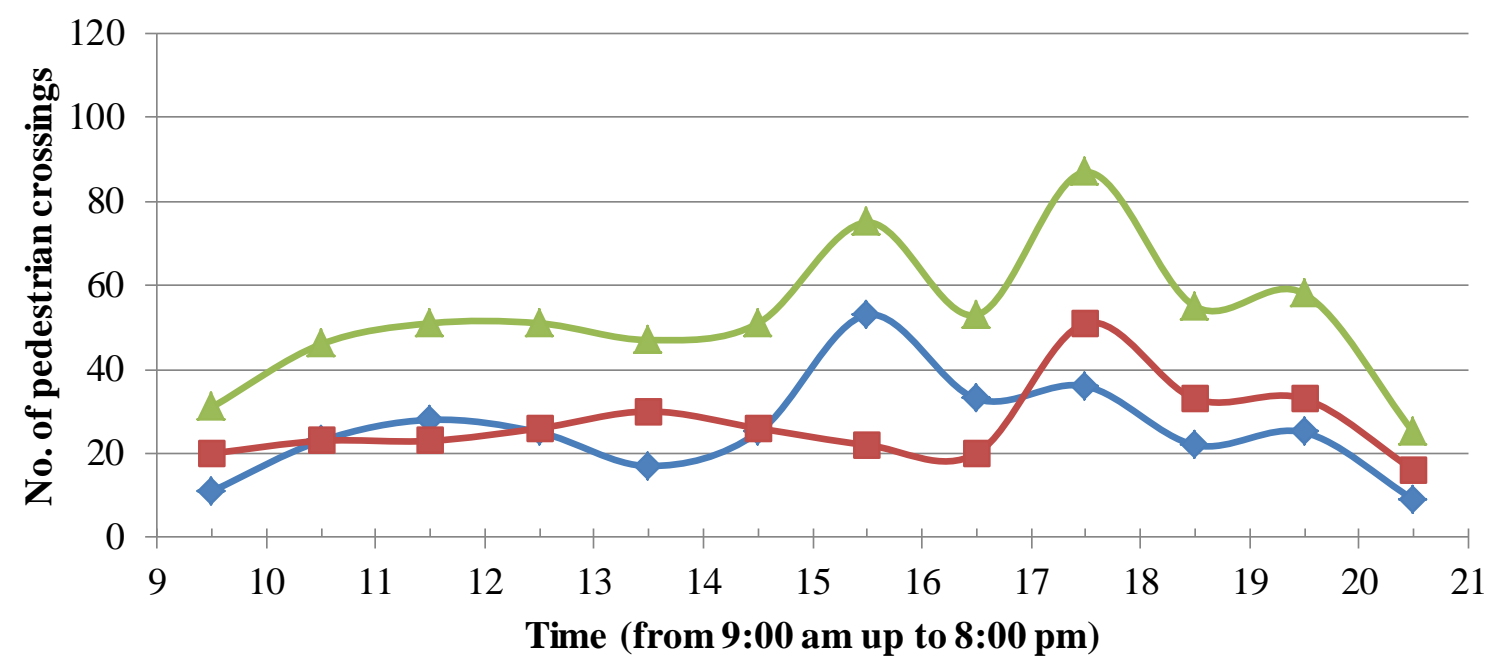

$\longrightarrow$ Crossing pedestrians from entry lane $\longrightarrow$ Crossing pedestrians from exist lane $\longrightarrow$ All crossing pedestrians

(a)

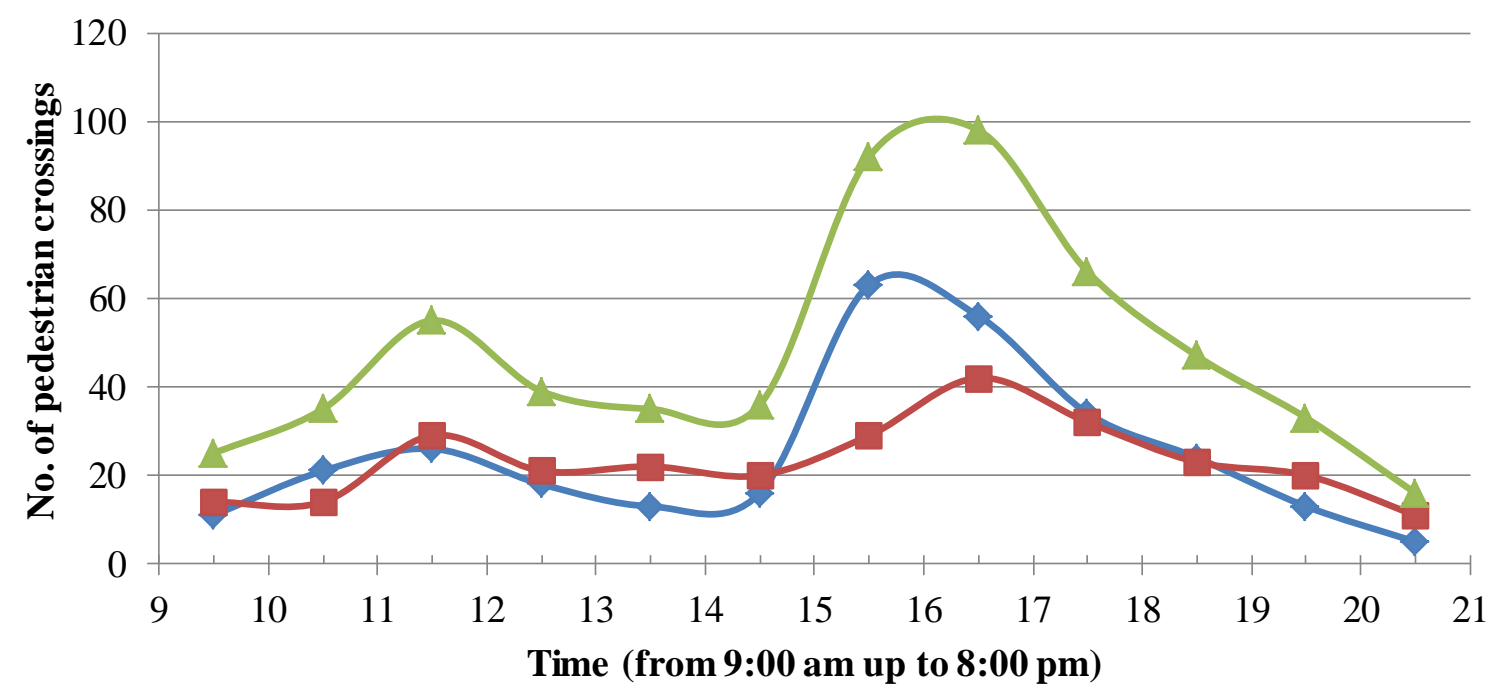

$\longrightarrow$ Crossing pedestrians from entry lane $\longrightarrow$ Crossing pedestrians from exist lane $\longrightarrow$ All crossing pedestrians

(b)

Figure B.2 Crossing pedestrians at approach B beginning from entry lane and from exit lane from 9:00 am to 8:00 pm a) Day one, and b) Day two 


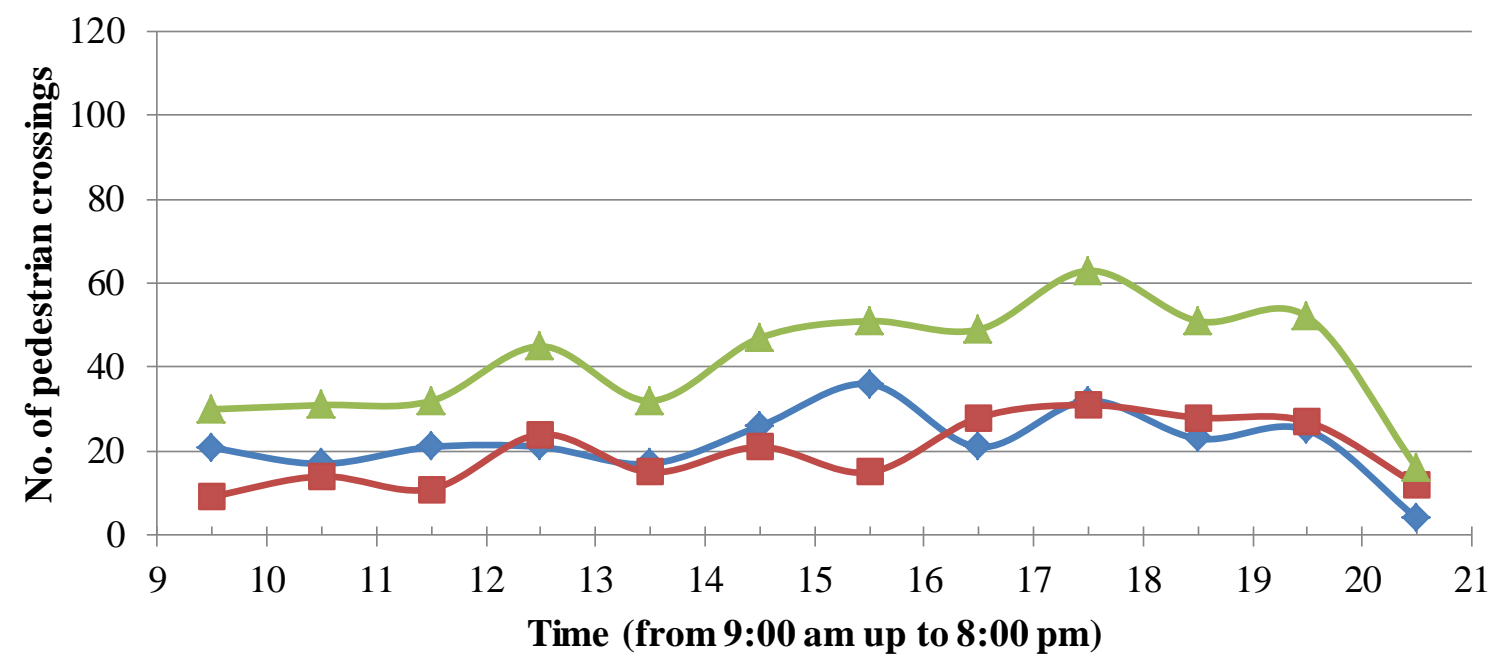

$\longrightarrow$ Crossing pedestrians from entry lane $\longrightarrow$ Crossing pedestrians from exist lane $\longrightarrow$ All crossing pedestrians

(a)

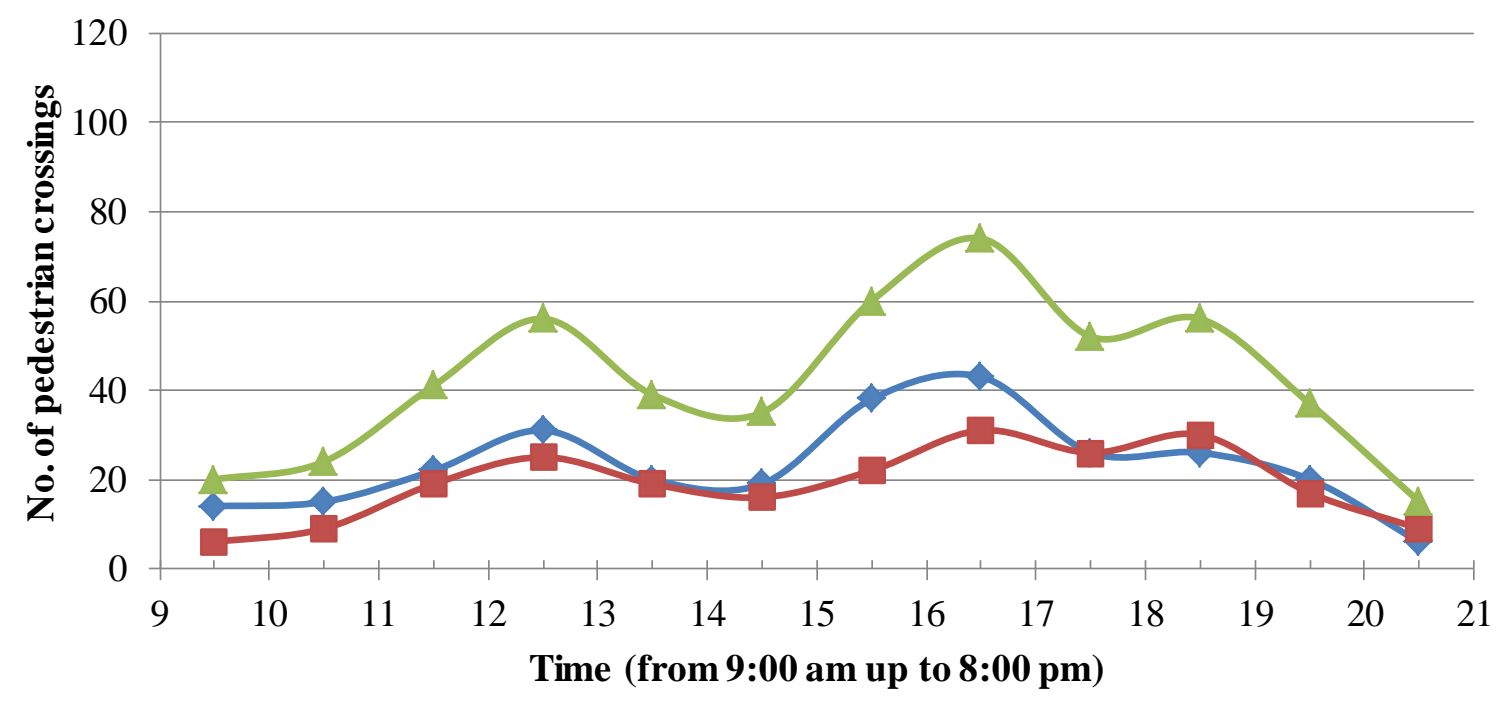

$\longrightarrow$ Crossing pedestrians from entry lane $\longrightarrow$ Crossing pedestrians from exist lane $\longrightarrow$ All crossing pedestrians

(b)

Figure B.3 Crossing pedestrians at approach $\mathrm{C}$ beginning from entry lane and from exit lane from 9:00 am to 8:00 pm a) Day one, and b) Day two 